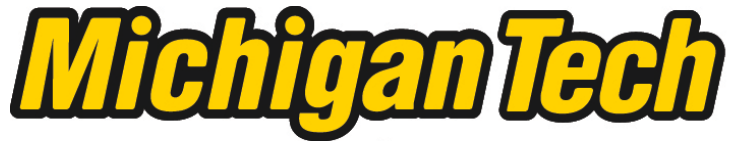 \\ Michigan Technological University Create the Future Digital Commons @ Michigan Tech
}

2013

\section{STUDIES OF FUNCTIONALIZED NANOPARTICLES FOR SMART SELF-ASSEMBLY AND AS CONTROLLED DRUG DELIVERY}

Xiaochu Ding

Michigan Technological University

Follow this and additional works at: https://digitalcommons.mtu.edu/etds

Part of the Biomedical Engineering and Bioengineering Commons, Nanoscience and Nanotechnology Commons, and the Polymer Chemistry Commons

Copyright 2013 Xiaochu Ding

\section{Recommended Citation}

Ding, Xiaochu, "STUDIES OF FUNCTIONALIZED NANOPARTICLES FOR SMART SELF-ASSEMBLY AND AS CONTROLLED DRUG DELIVERY", Dissertation, Michigan Technological University, 2013.

https://doi.org/10.37099/mtu.dc.etds/779

Follow this and additional works at: https://digitalcommons.mtu.edu/etds

Part of the Biomedical Engineering and Bioengineering Commons, Nanoscience and Nanotechnology Commons, and the Polymer Chemistry Commons 


\title{
STUDIES OF FUNCTIONALIZED NANOPARTICLES FOR SMART SELF-ASSEMBLY AND AS CONTROLLED DRUG DELIVERY SYSTEM
}

\author{
By \\ Xiaochu Ding \\ A DISSERTATION \\ Submitted in partial fulfillment of the requirements for the degree of \\ DOCTOR OF PHILOSOPHY \\ In Chemistry
}

MICHIGAN TECHNOLOGICAL UNIVERSITY

2013

(C)2013 Xiaochu Ding 
This dissertation has been approved in partial fulfillment of the requirements for the Degree of DOCTOR OF PHILOSOPHY in Chemistry.

Department of Chemistry

Dissertation Advisor: $\quad$ Patricia A. Heiden

Committee Member: $\quad$ Shiyue Fang

Committee Member: $\quad$ Ashutosh Tiwari

Committee Member: $\quad$ Tolou Shokuhfar

Department Chair: Cary Chabalowski 


\section{Table of Content}

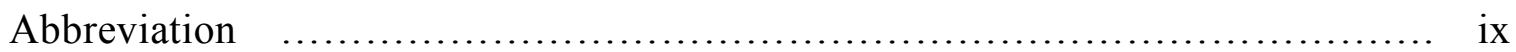

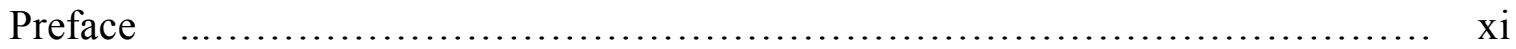

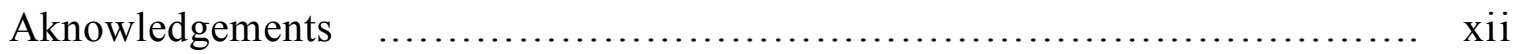

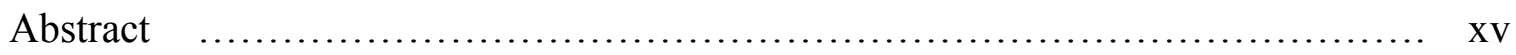

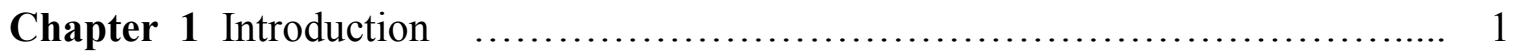

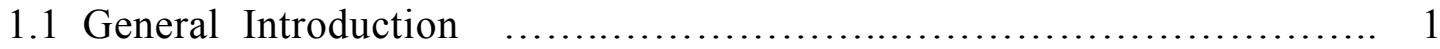

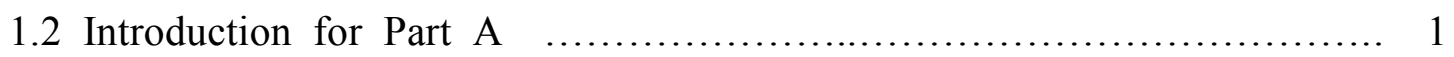

1.2.1 Background on Polymeric Hydrogel Scaffolds and Their Limitation $\quad \ldots \quad 2$

1.2.2 Self-Assembling Peptide Scaffolds and Their Limitation $\ldots \ldots \ldots \ldots \ldots .4$

1.2.3 Nanocomposite Hydrogel Scaffolds and Their Limitation $\quad \ldots . \ldots \ldots \ldots . . .5$

1.2.4 Self-Assembled Peptide-Nanoparticle Precedents $\quad \ldots \ldots \ldots \ldots \ldots \ldots \ldots . . . \ldots$

1.2.5 Rationale and Advantages of Research Performed in Part A .......... 8

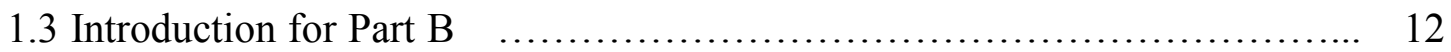

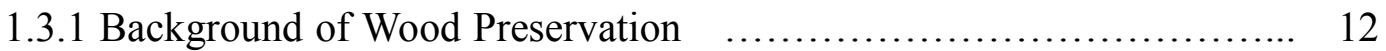

1.3.2 Rationale for Controlled Release of Organic Wood Preservatives ..... 14

1.3.3 Rationale for Study of Copper Oxide Nanoparticle Leaching .......... 16

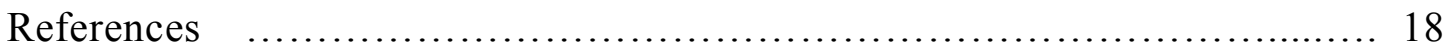

Chapter 2 Studies of Novel Self-Assembly Structures from Peptide-Functionalized Polymeric Micro- and Nanoparticles for Tissue Engineering $\quad \ldots \ldots \ldots \ldots \ldots \ldots \ldots \ldots . \ldots 28$

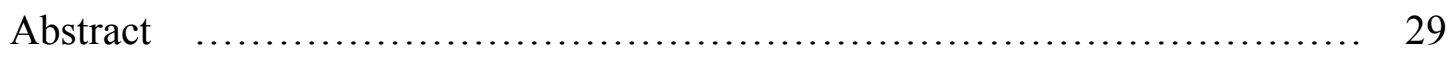

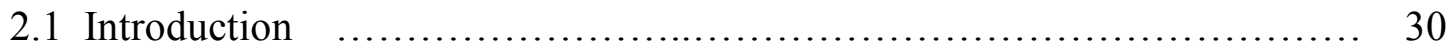

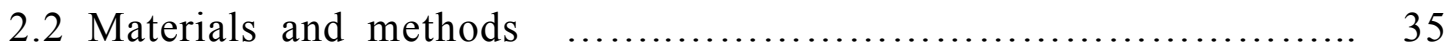

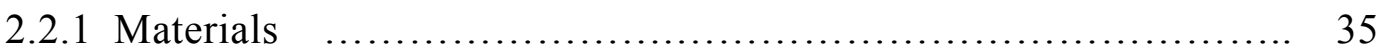

2.2.2 Synthesis of peptides (P1 and P2) bearing opposite charges $\ldots . . . . . \quad 35$

2.2.3 Synthesis of $\mathrm{S}, \mathrm{S}^{\prime}$-bis $\left(\alpha, \alpha^{\prime}\right.$-dimethylacetic acid) trithiocarbonate $\ldots . .38$ 
2.2.4 Synthesis of hydrophilic block (Macro-CTA) and amphiphilic tri-block

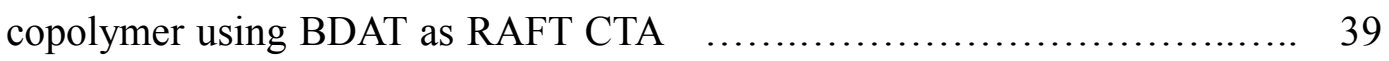

2.2.5 Characterization of hydrophilic blocks and tri-block copolymers $\ldots . .41$

2.2.6 Coupling of peptide to triblock copolymer $\quad \ldots \ldots \ldots \ldots \ldots \ldots \ldots \ldots \ldots, 43$

2.2.7 Self-assembly of peptide-copolymer conjugates in phosphate buffered saline

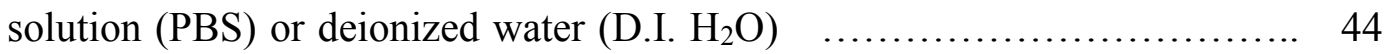

2.2.8 Controlled release of Insulin from assembled nanoparticles $\quad \ldots . \ldots \ldots \ldots .45$

2.2.9 MTS cytotoxicity assay using SW-620 cell lines $\ldots \ldots \ldots \ldots \ldots \ldots . \ldots 4$

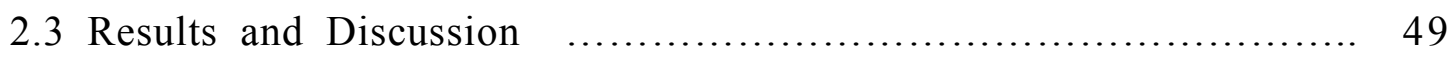

2.3.1 Design of peptides (P1 and P 2) for $\beta$-sheet self-assembly $\quad \ldots . . . \ldots . . .49$

2.3.2 Synthesis and characterization of amphiphilic tri-block copolymers ... 53

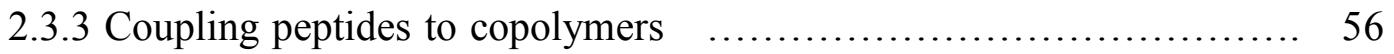

2.3.4 Self-assembly of peptide-copolymer conjugates in aqueous solution ... 57

2.3.5 Controlled release study of Insulin as model drug $\quad \ldots \ldots \ldots \ldots \ldots \ldots \ldots .61$

2.3.6 Biocompatibility study on SW-620 cell lines by MTS assay $\quad \ldots \ldots \ldots \ldots .64$

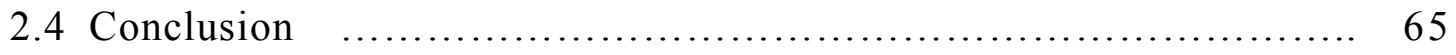

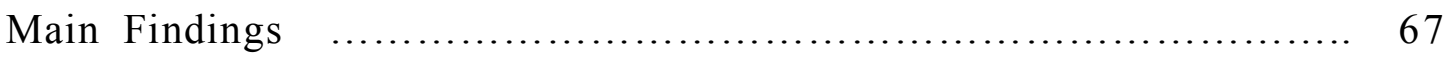

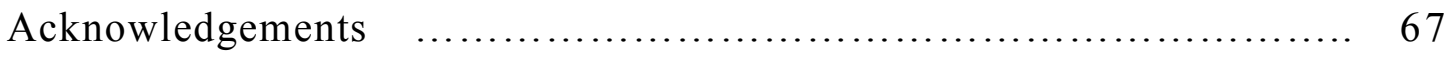

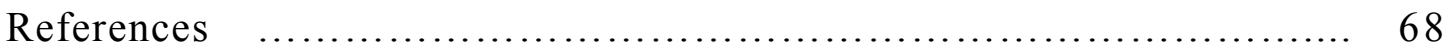

Chapter 3 Smart Self-Assembly of Peptide-Functionalized Amphiphilic Copolymer for Nanoparticle Fibers and 3D Scaffolds with Multi-drug Loading and Controlled

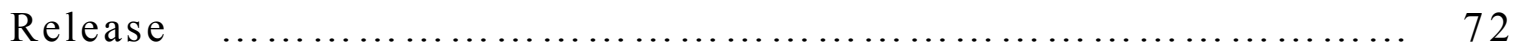

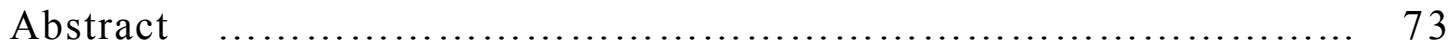

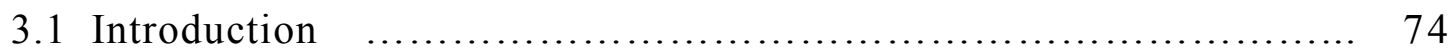

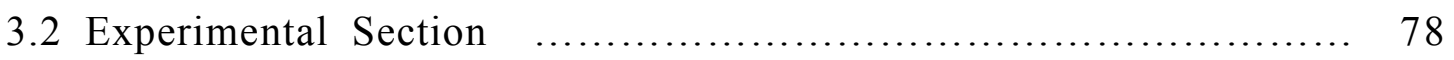

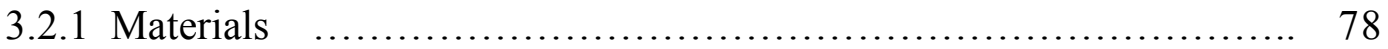


3.2.2 Synthesis and self-assembly of ionic complementary peptides

3.2.3 Synthesis of amphiphilic triblock copolymers with reactive terminals by

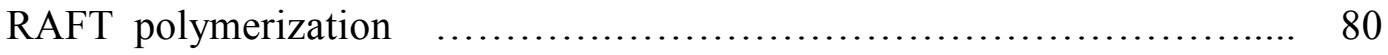

3.2.4 Characterization of hydrophilic block and tri-block copolymers $\ldots \ldots .81$

3.2.5 Coupling reaction of copolymer with P1 and P2 $\ldots \ldots \ldots \ldots \ldots \ldots \ldots . . . . . . .2$

3.2.6 Self-assembly study of peptide-copolymer conjugates in aqueous

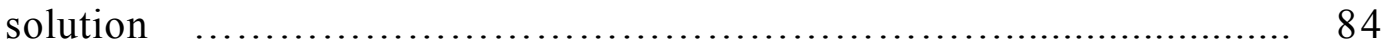

3.2.7 Self-assembly study of peptide-functionalized nanoparticles with additional

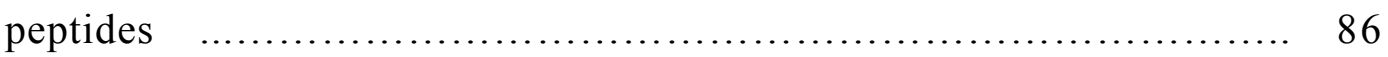

3.2.8 Controlled release test with hydrophobic fluorescein as model drug .... 87

3.2.9 Multiple-drug loading and controlled release test using nitrofurazone and

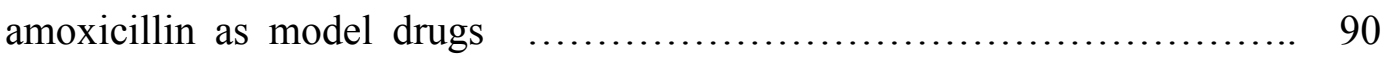

3.2.10 Cytotoxicity test on NIH3T3 cell lines $\quad \ldots \ldots \ldots \ldots \ldots \ldots \ldots \ldots \ldots . \ldots . \ldots \ldots$

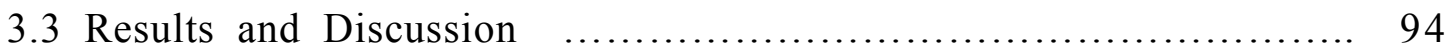

3.3.1 Rationale for peptide design and study of peptide self-assembly ........ 94

3.3.2 Synthesis of amphiphilic copolymer with different ratio of hydrophilic and

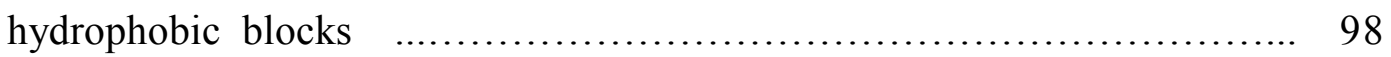

3.3.3 Coupling reaction of copolymer with peptides $\quad \ldots \ldots \ldots \ldots \ldots \ldots \ldots . . \ldots 101$

3.3.4 Self-assembly study of peptide-functionalized nanoparticles $\quad \ldots \ldots \ldots . .104$ 3.3.5 Self-assembly study of peptide-functionalized nanoparticle with additional

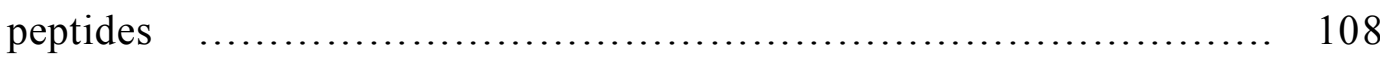

3.3.6 Controlled release test of multi-drug models $\quad \ldots \ldots \ldots \ldots \ldots \ldots \ldots \ldots . . \ldots 112$

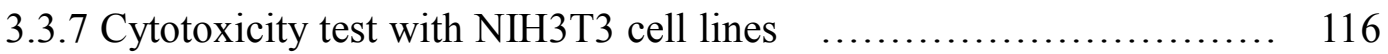

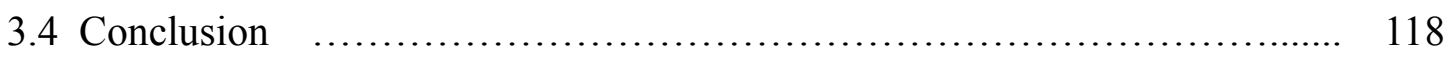

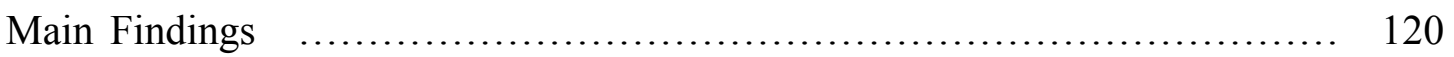

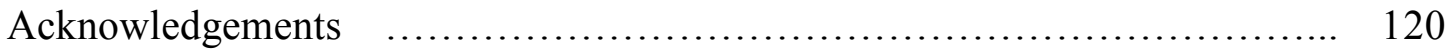


References

Chapter 4 Efficient One-Pot Synthesis and Loading of Self-Assembled Amphiphilic Chitosan Nanoparticles for Low-Leaching Wood Preservation

Abstract

4.1 Introduction

4.2 Experimental Part

4.2.1 Materials

4.2.2 Preparation of Nanoparticles

4.2.3 Nanoparticle Size

4.2.4 Nanoparticle Composition

4.2.5 Tebuconazole Content in Nanoparticles

4.2.6 Delivery Efficiency into Wood Blocks

4.2.7 Leaching Tests

4.2.8 GC-MS Analysis

4.2.9 Soil Jar Decay Test

4.3 Results and Discussion

4.3.1 Study of Reaction Conditions

4.3.2 Characterization of Fungicide-Containing Nanoparticles 138

4.4 Wood Leaching and Wood Preservation

4.4.1 Nanoparticle Delivery into Wood Blocks

4.4.2 Leaching from wood blocks

4.4.3 Wood Preservation Efficacy by Soil Jar Decay Test

4.5 Conclusion

Main Findings

Acknowledgements

References 
Chapter 5 Comparing Leaching of Different Copper Oxide Nanoparticles and Ammoniacal Copper Salt from Wood ..................................... 152

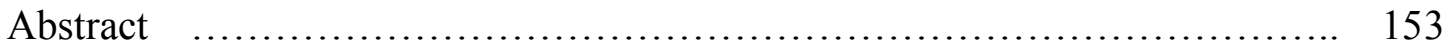

5.1 Introduction .................................................... 154

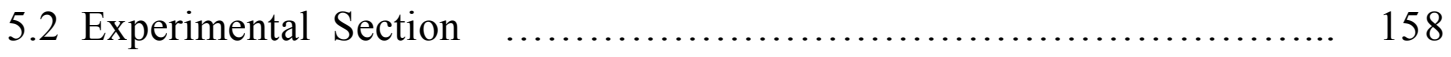

5.2.1 Materials (..................................................... 158

5.2.2 Preparation of Water-Soluble Modified Chitosan ......... 158

5.2.3 Preparation of Polymer-Stabilized and Unstabilized CuO

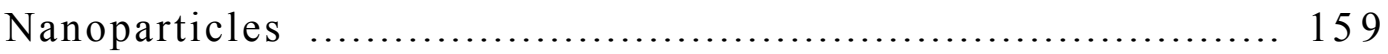

5.2.4 Preparation of ACQ _............................................. 161

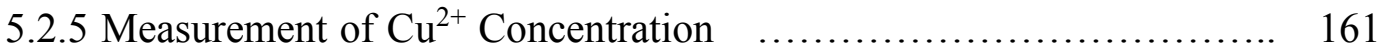

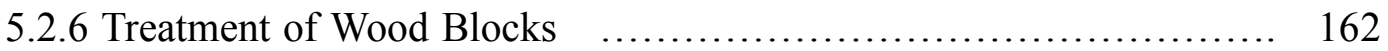

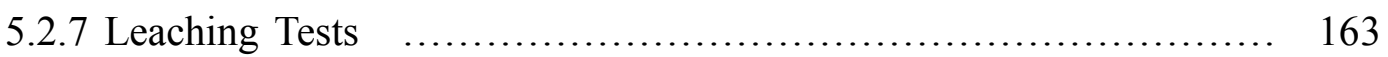

5.2.8 Soil Jar Decay Test ............................................... 164

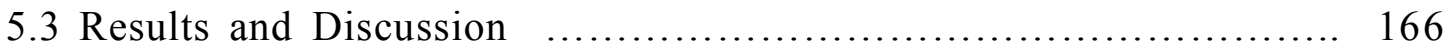

5.3.1 Preparation and Characterization of Polymer-Stabilized $\mathrm{CuO}$ Nanoparticles $\quad$................................................... 166

5.3.2 Nanoparticle and ACQ Retention in Wood Blocks .............. 167

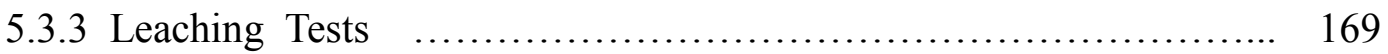

5.3.4 Biological Efficacy against G. trabeum in Soil Jar Decay Tests $\quad \ldots . . .173$

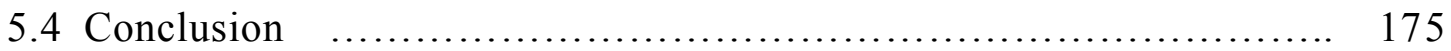

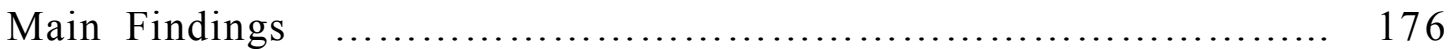

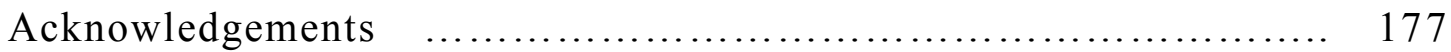

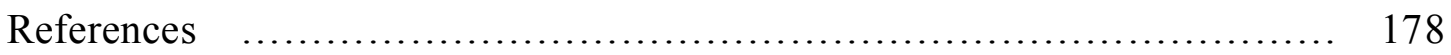

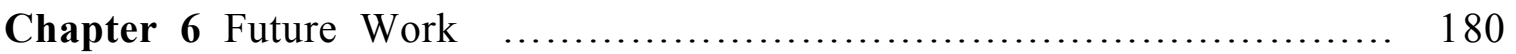

Appendix A Supplementary Data for Chapter 2 ............................. 184 


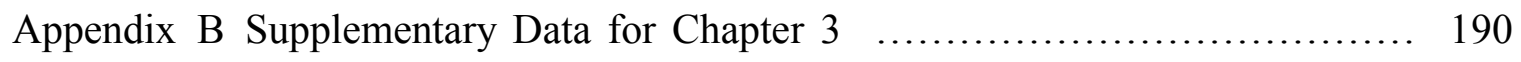

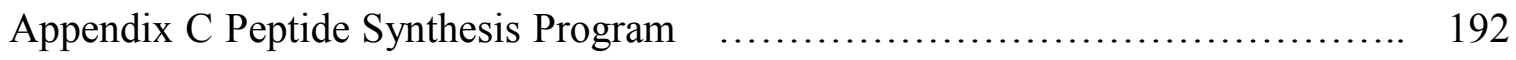

Appendix D Permission License Number for Chapter $4 \quad \ldots \ldots \ldots \ldots \ldots \ldots \ldots \ldots \ldots . \ldots \ldots$

Appendix E Permission License Number for Chapter $5 \quad \ldots \ldots \ldots \ldots \ldots \ldots \ldots \ldots . \ldots 211$ 


\section{Abbreviation List}

\section{Peptide Abbreviations:}

AEAK16-II:Alanine-glutamic acid-alanine-lysine-alanine-glutamic acid-alaninelysine-alanine-glutamic acid-alanine-lysine-alanine-glutamic acid-alanine-lysine

Ac-EWEVEVEVEV-amide: Acetic acid-glutamic acid- tryptophan- glutamic acidvaline-glutamic acid-valine-glutamic acid-valine-glutamic acid-valine-amide

Ac-WKVKVKVKVK-amide: Acetic acid-tryptophan-lysine-valine-lysine-valinelysine-valine-lysine-valine-lysine- amide

$\mathrm{H}_{2} \mathrm{~N}$-TTTT-AEAEAEAE-amide: $\mathrm{H}_{2} \mathrm{~N}$-Threonine -Threonine -Threonine -Threonine -Alanine -Glutamic acid -Alanine -Glutamic acid -Alanine -Glutamic acide -Alanine -Glutamic acid-amide

$\mathrm{H}_{2} \mathrm{~N}$-TTTT-AKAKAKAK-amide: $\quad \mathrm{H}_{2} \mathrm{~N}$-Threonine-Threonine-Threonine-ThreonineAlanine- Lysine-Alanine-Lysine-Alanine-Lysine-Alanine-Lysine-amide

LELC16: Leucine -glutamc acid -leucine -cysteine -leucine -glutamic acid -leucine -cysteine -leucine -glutamic acid -leucine -cysteine -leucine -glutamic acid -leucine -cysteine

RADA16-I: Arginine- alanine- aspartic acid-alanine- arginine- alanine- aspartic acidalanine- arginine- alanine- aspartic acid- alanine- arginine- alanine- aspartic acid -alanine

\section{Other Abbreviations:}

ACQ: Alkaline copper quaternary

AIBN:2,2'-azobisisobutyronitrile

BDAT:S,S'-bis $\left(\alpha, \alpha^{\prime}\right.$-dimethylacetic acid

CCA: Chromated copper arsenate

CTA: Chain transfer agent

C2M: Chitosan-g-poly(methyl methacrylate) 
CsA: CyclosporinA

DMF: N,N-Dimethylformamide

DMSO: Dimethyl sulfoxide

DIPEA: N,N-diisopropylethylamine

GC-MS: Gas chromatography-mass psectrometry

GF: Growth factor

HAMC: Hyaluronan-methylcellulose

HATU: 2-(7-aza-1-H-benzotriazol-1-yl)-1,1,3,3-tetramethylaminium

HEA: 2-Hydroxyethyl acrylate

HEMA: 2-Hydroxyethyl methacrylate

HTCC: [(2-hydroxy-3-trimethylammonium)propyl] chitosan

MMA: methyl methacrylate

MALDI-TOF MS: Matrix-assisted laser desorption/ionization time-of-flight mass spectrometry

PEG: Poly(ethylene glycol)

PHEMA: Poly(hydroxide ethyl methacrylate)

PHEA: Poly(hydroxide ethyl acrylate)

PIP: Piperidine

PLA: Poly(lactic acid)

PLGA: Poly(lactic-co-glycol acid)

PMMA: Poly (methyl methacrylate)

PVP: Poly(vinyl pyrrolidone)

RAFT: Reversible addition fragmentation chain transfer

TFA: Trifluoroacetic acid

TMS: Tetramethylsilane

VP: 1-vinyl-2-pyrrolidinone 


\section{Preface}

This dissertation is in the form of a collection of published or submitted manuscripts. The initial ideas for the research projects in these articles were conceived by Prof. P. A. Heiden, but the synthetic designs and characterizations, and decisions made on how to advance the project to achieve the objectives were those of Xiaochu Ding, Ph.D. Candidate. In chapter 2, the biocompatibility tests on SW-620 cell lines were contributed by Dr. Jagadeesh Janjanam (Dr. Ashutosh Tiwari's group, Department of Chemistry). In chapter 3, the biocompatibility tests on NIH3T3 cell lines were contributed by Mr. Trevor Moser (Dr. Tolou Shokuhfar's group, Department of Mechanical Engineering and Engineering Mechanics). FESEM and TEM characterization was conducted by Mr. Qi Gao (Dr. Raze ShahbazianYassar's group, Department of Mechanical Engineering and Engineering Mechanics) with help of Mr. Owen P. Mills (Senior Research Engineer and Scientist II, Department of Materials Science and Engineering). In chapter 4 and 5, soil jar decay tests were contributed and conducted by Dr. Dana L. Richter (School of Forest Resources and Environmental Science). TEM and FESEM microscopy were conducted by Mr. Owen P. Mills and Dr. Ning Chen respectively. In chapter 5, part of the leaching test was contributed by Ms. Soha Albukhari (Ph.D. candidate, Department of Chemistry) and Dr. Martha Barajas Meneses (Department of Chemistry). 


\section{Acknowledgements}

I would like to give my most sincere appreciations to my advisor, Dr. Patricia A. Heiden, as one of the most knowledged and respectful professors I have known and have the honor to work with her in the last 5 years. Dr. Heiden always welcomes her students to conceive their own ideas and conduct researches independently, and also guide her students how to be a team member when cooperation is needed. Dr. Heiden is teaching and guiding students to become qualified researchers by enlightening them through valuable references, book knowledge, communication and inspiration from nature, leaving a large space for students to conceive and conduct research according to their own understanding, and then giving them the necessary correction and guidance. Such educating method not only develops students the ability to absorb knowledge, but most importantly to digest knowledge and use knowledge, so that students are able to become a self-motivated and independent researcher.

I also sincerely thank my committee members, Dr. Shiyue Fang, Dr. Ashutosh Tiwari and Dr. Tolou Shokuhfar, for their great help for my research, my dissertation writing and my defense.

I gratefully appreciate the financial supports from American Environmental Agency Science to Achieve Results (STAR) Program (Grant Number GR832371) and Department of Chemistry (Michigan Technological University), which give me more time to focus on research. Other various financial supports are also greatly thankful, including Outstanding Graduate Student Fellowship (Department of Chemistry, Summer 2011), Doctoral Finishing Fellowship (Graduate School, Spring 2013), Travel Grants from Biotech Research Center (BRC) and Graduate School (Michigan Technological University, for ACS $245^{\text {th }}$ National Meeting, 2013).

During last 5 years, I also received various help from other faculty members and staffs at Department of Chemistry to give me many instrumention and analysis expertise 
to assist my research. Without their help, I could not have conducted my research effectively to complete my P.h.D. program. I greatly appreciate these people and their help. They are Dr. Martin Thompson (peptide synthesizer, freeze-dryer and peptide synthesis techniques), Dr. Sarah Green (Fluorescence Spectrometer), Dr. David Chesney (HPLC and training), Dr. Ashutosh Tiwari and Dr. Tarun K. Dam (Centrifuge), Dr. Shiyue Fang (Organic synthesis technique), Mr. Jerry Lutz (GC-MS and NMR training), Mr. Andrew Galerneau (MALDI-TOF MS and FT-IR training), Mr. Dean Seppala (Facility maintenance). All other staffs from Department of Chemistry also gave me valuable help, I would like to express my sincere appreciations to them.

It's my great honor to have the opportunities to collaborate with various scientists outside the field of chemistry. Here I would like to express my acknowledgements to Dr. Dana L. Richter (Soil Jar Decay Test for Wood Preservation, School of Forest Resources and Environmental Science), Dr. Ashutosh Tiwari's group and Dr. Jagadeesh Janjanam (Department of Chemistry, Biocompatibility tests on SW-620 cell lines), Dr. Tolou Shokuhfar's group and Mr. Trevor Moser (Department of Mechanical Engineering and Engineering Mechanics, Biocompatibility tests on NIH3T3 cell lines), Dr. Raze ShahbazianYassar's group (Department of Mechanical Engineering and Engineering Mechanics, for AFM and FESEM help). I especially thank Mr. Qi Gao and Mr. Owen P. Mills for FESEM and TEM training and helping.

The successful progress of my research is also partially from the valuable help of my labmates: Dr. Ganesh Kumar Arumugam, Dr. Ning Chen, Ms. Soha Albukhari, Dr. Martha Barajas Meneses and Mr. Xu Xiang, and many of my friends: Dr. Qili Hu, Ms. Katrina Bugielski, Ms. Nazmiye Bihter Yapici, Mr. Durga Pokharel. And the help from all other graduate students is also sincerely appreciated.

Finally, I would like to thank my families' support for my study of Ph. D. program at Michigan Tech University. I also would like to express thanks to my previous advisor and 
numerous good friends although I haven't mentioned their names. With their help and support, I'm progressing. 


\begin{abstract}
This dissertation is related to the studies of functionalized nanoparticles for self-assembly and as controlled drug delivery system. The whole topic is composed of two parts. In the first part, the research was conducted to design and synthesize a new type of ionic peptide-functionalized copolymer conjugates for self-assembly into nanoparticle fibers and 3D scaffolds with the ability of multi-drug loading and governing the release rate of each drug for tissue engineering. The self-assembly study confirmed that such peptide-functionalized amphiphilic copolymers underwent different self-assembly behavior. The bigger nanoparticles were more easily assembled into nanoparticle fibers and 3D scaffolds with larger pore size, while the smaller nanoparticle underwent faster self-assembly to form more compact 3D scaffolds with smaller porosity but more stable structure. Controlled release studies confirmed the ability of governing simultaneous release of different model drugs with independent release rate from a same scaffold. Cytotoxicity tests showed that all synthesized peptides, copolymers and peptide-copolymer conjugates were biocompatible with SW-620 cell lines and NIH3T3 cell lines. This new type of self-assembled scaffolds combined the advantages of peptide nanofibers and versatile controlled release of polymeric nanoparticles to achieve simultaneous multi-drug loading and controlled release of each drug, uniform distribution and flexibility of hydrogel scaffolds.

The investigations in second part were first to design and synthesize organic biocide-loaded nanoparticles for low-leaching wood preservation using a cost-effective one-pot method to synthesize amphiphilic chitosan-g-PMMA nanoparticles loading with 25-28 wt.\% of the fungicide tebuconazole with particle size of $\sim 100 \mathrm{~nm}$ diameter by FESEM. FESEM analysis confirmed efficient penetration of nanoparticles throughout the treated wooden stake with dimension of $19 \times 19 \times 455 \mathrm{~mm}$. Leaching studies showed that biocide introduced into sapwood via nanoparticles leached only $\sim 9 \%$ compared with the
\end{abstract}


amount leached from tebuconazole solution-treated control, while soil jar tests showed that the nanoparticle-treated wood blocks were effectively protected from biological decay tested against G. trabeum, a brown rot fungus.

Copper oxide nanoparticles with and without polymer stabilizers were also investigated to use as inorganic wood preservatives to clarify the factor affecting copper leaching from treated wood. Copper oxide nanoparticles with uniform diameters of $\sim 10$ $\mathrm{nm}$ and $\sim 50 \mathrm{~nm}$ were prepared, and the leachates from southern pine sapwood treated with these nanoparticles were analyzed. It was found by TEM and EDS analysis that significant numbers of nanoparticles leached from the treated wood. The $50 \mathrm{~nm}$ nanoparticles leached slightly less than a soluble copper salt control, but $10 \mathrm{~nm}$ nanoparticles leached substantially more than the control. The effect of polymer stabilizers on nanoparticle leaching was also investigated. Results showed that polymer stabilizers increased leaching. The trends showed that nanoparticle size was a major factor in copper leaching. 


\section{Chapter 1 Introduction}

\subsection{General Introduction}

This dissertation is a collection of already-published articles or articles that are being prepared for submission. It is comprised of two parts, with both parts being related to design and synthesis of functionalized nanoparticle for self-assembly and for controlled drug delivery systems. The first part describes the synthesis and fabrication of a new type of ionic complementary peptide-functionalized amphiphilic copolymer designed to self-assemble into sophisticated polymeric nanoparticle fibers and 3D scaffolds that have the ability to simultaneously load multiple drugs and to separately govern the release rate of each drug for appropriate release levels. The methodology could be applied for various applications but here the intended application was for tissue engineering. The second part of this dissertation describes the synthesis and testing of biocide-loaded nanoparticles for use in low leaching wood preservation. Each dissertation part has its own features and considerations for design and application, but also has common features with respect to nanoparticles as controlled drug delivery system. This chapter will introduce the background information, rationale, and perceived advantages of the materials investigated in this dissertation.

\subsection{Introduction for Part A}

The objective of Part A is to fabricate an advanced self-assembled nanoparticle fiber scaffolding system with the following advantages: (1) versatility so that different drugs can be introduced into nanoparticle matrices that are appropriate to each drug so release rates can be appropriately governed; (2) to control assembly of the individual nanoparticles into a fiber scaffold; (3) to control the sequence and spatial arrangement of the drug-loaded nanoparticles in the scaffold; (4) study further self-assembly into a 3D scaffolding system; and (5) demonstrate the ability to combine the advantages of 
controlled release of multiple drugs with biocompatible tissue scaffolds, and retain these abilities in an injectable hydrogel scaffold system.

Such a polymeric nanoparticle fiber has substantial advantages over electrospunnanofibers, traditional hydrogels, and nanofiber/hydrogel blends. To the best of our knowledge, such polymeric nanoparticle fibers and 3D scaffolds are a fundamentally new approach to self-assembly systems, and are reported here for thefirst time.

\subsubsection{Background on Polymeric Hydrogel Scaffolds and Their Limitation}

Artificial scaffolds, such as hydrogels and nanofibers, have been under extensive investigations for biological mimic of extracellular matrix to increase cell survival and also guide cell activities to improve tissue regeneration (1-3). Many studies have shown that effective tissue regeneration is usually dependent on delivery of multiple drugs and other active ingredients, such as growth factors, to the cells within the damaged tissue region (4). For example,effective skin regeneration usually requires a complex set of growth factors and cytokines, such as fibroblast growth factor, keratinocyte growth factor, vascular endothelial growth factor and interleukin $1 \alpha$, to be delivered to and release within the damaged region to promote cell proliferation and migration to achieve wound healing $(5,6)$. Another example is regeneration of neural tissue of the retina, which is a light sensitive layer of tissue lying in the inner surface of the eye. The retina itself consists of multiple cellular layers across a depth of 100-130 $\mu \mathrm{m}$ (7). Studies show that successful retina tissue regeneration requires a scaffold having the ability to simultaneously localize multiple growth factors (i.e. amino-terminal sonic hedgehog for rod photoreceptor cells and ciliaryneurotrophic factor for bipolar cells) to promote the differentiation of the retinal precursor cells to mature cells (8). Therefore, it is clear that the tissue regeneration significantly benefits from scaffolding systems with advanced drug delivery abilities for multiple drugs. 
Conventional polymeric hydrogels, whether from synthetic or natural polymers, have been widely studied as scaffold systems, with and without incorporated drugs, for tissue regeneration. For example, bioactive ingredients have been physically or chemically incorporated in synthetic hydrogels such as poly(ethylene glycol) (PEG) hydrogels (9, 10),poly(lactide)-b-poly(ethylene oxide)-b-poly(lactide) (PLA-b-PEO-b-PLA) or poly(lactic-co-glycol acid) (PLGA) (11,12), and natural polymer hydrogels, e.g. alginate, collagen, chitosan, gelatin (13-19). But most of these traditional scaffolds can accommodate only a single drug and have limited ability to control the drug release rate.

Multi-drug loading in polymeric hydrogels has been reported, but as drug delivery systems and not a tissue scaffold (20-22). For example, Salmaso et al. (21) simultaneously loaded different model drugs in cyclodextrin-PEG hydrogels by swell embedding. The maximal incorporation by this method for lysozyme, $\beta$-estradiol, and quinine was 2, 0.6 and $2.4 \mathrm{wt} . \%$ respectively, but there was little ability to governing the release of each drug, which had different physical properties. Lysozyme was quickly released, while $\beta$-estradiol and quinine release was inversely proportional to the cyclodextrin/PEG ratio. The release profiles were significantly affected by interaction of the drugs with hexamethylated $\beta$-cyclodextrins in the hydrogel matrices. Such release profiles imply the non-effective distribution of the loaded drugs in hydrogels. If such a material is used as a multi-drug loaded hydrogel for tissue regeneration, the non-uniform drug distribution and poor drug release profiles will not be able to guide cell activity and could be detrimental.

Scaffolding with controlled delivery of multiple growth factors (GFs) for tissue engineering, including hydrogels, microsphere gels, and polymeric micro/nanoparticle encapsulations, have also been studied and reviewed (23). Typical methods for incorporation of multiple GFs include: combining multi-layer hydrogels with each layer loaded with one type of growth factor, or hydrogel loading coupled with microsphere 
loading, or hydrogel loading with micro/nanoparticle loading (23). These techniques each have their own advantages with respect to their ability to load different GFs, and also showed some control over the release rate of the loaded GFs, but these methods do not allow control over the placement and distribution of each micro/nanoparticle.

\subsubsection{Self-Assembling Peptide Scaffolds and Their Limitation}

Self-assembled peptide nanofiber scaffolds have increased in popularity for tissue engineering due to non-toxicity, biodegradability, and because the micro-environment it provides may be similar to the extracellular matrix for cell attachment and in situ formation of a hydrogel under physiological conditions (24). A typical ionic complementary $\beta$-sheet peptide AEAK16-II (AEAEAKAKAEAEAKAK) was first discovered in a yeast protein, zuotin (25). This type of ionic complementary peptide shows a spontaneous self-assembly into stable $\beta$-sheets in aqueous conditions, across a broad range of temperature, and a wide $\mathrm{pH}$ range, and the scaffold can maintain integrity in the presence of high concentrations of the denaturing agents of urea and guanidium hydrochloride $(\mathbf{2 6 , 2 7})$.

The driving forces for self-assembly of the peptides are the hydrophobic interactions of alanine (A) domains and the ion-pair interactions between negatively charged glutamic acid (E) side chains and positively charged lysine (K) side chains. The strong ion-pair interactions contribute to stable $\beta$-sheet formation, which was further supported on studying another pair of designer peptides, i.e. self-repulsive but mutually attractive peptide sequences that possessed positive charges (Ac-WKVKVKVKVK-amide) and negative charges (Ac-EWEVEVEVEV-amide) (28). On mixing this pair of ionic complementary peptides, a rapid assembly into a viscoelastic hydrogel occurred at a concentration as low as $0.25 \mathrm{wt} . \%$. This hydrogel retained mechanical strength, even after repeated shear-induced breakdowns, due to the electrostatic interactions. The strong electrostatic and selective interactions between the opposite charges proved to be one of 
the key merits of using ion-complementary $\beta$-sheet peptides.

Self-assembling peptide nanofibers using designed (i.e. not naturally occurring) peptides (e.g. EAK16-II, RADA16-I or II) have been widely used as both controlled drug delivery systems and 3D scaffolds (29,30). This is typically done by physically incorporating a desired drug into the peptide scaffold during gel formation $(\mathbf{3 1}, \mathbf{3 2})$, or by chemically bonding it onto the $\mathrm{C}$-terminal or $\mathrm{N}$-terminal of the peptide (33). Sometimes a combination of both methods is used to load multiple active agents $(\mathbf{3 4 , 3 5})$. However, these methods still have significant limitations with respect to the quantity of drug(s) to be incorporated, and the properties of drug(s) may have an impact on the subsequent self-assembly process or the mechanical stability of the formed hydrogel. Therefore, this approach is very limited in its ability to control the release of drugs with different properties within a same scaffold $(\mathbf{3 6 , 3 7 )}$. Moreover, effective distribution of multiple drugs in scaffolds is not always easy to achieve, so the peptide hydrogel itself must be designed in conjunction with the specific drug(s) that will be incorporated. Because of these limitations the self-assembling peptide scaffolds are either unsophisticated to satisfy the current requirements for tissue engineering applications.

\subsubsection{Nanocomposite Hydrogel Scaffolds and Their Limitation}

Because of the limitations of the scaffoldsystemsmentionedabove, researchers sought a more powerful and versatile technique to allow multiple drug incorporation within scaffolding systems so that the release rate of each drug could be controlled appropriately $(\mathbf{2 3 , 3 8 )}$. There are many ways to deliver drugs, such asmicelles, colloids, capsules, and solid polymeric nanoparticles, including self-assembled core-shell nanoparticles $(\mathbf{3 9 , 4 0 )}$. Solid polymeric nanoparticles have been used for oral drug delivery (41), epidermal injection (42), targeted delivery of anticancer drugs to cancer cells (43), and delivery of bioactive drugs for central nervous system regeneration $(44,45)$. Core-shell polymeric nanoparticles are particularly attractive for use in tissue scaffolding because the core can be 
designed to be suitable for a desired hydrophilic or hydrophobic drug while the shell can be designed to be suitable for the use environment. Also, core-shell nanoparticles are relatively easy to prepare with a controlled particle size, and various biocompatible hydrophilic and hydrophobic monomers are commercially available to make block copolymers that self-assemble into core-shell nanostructures (46).

Even though polymeric nanoparticles have been used as versatile controlled delivery system for various drugs, the nanoparticle itself lacks scaffolding property to guide cell activities for tissue regeneration.

In recent years, nanocomposite hydrogels have been studied. In these hydrogels drug-containing nanoparticles have been blended into hydrogels to form a new generation of scaffolds for tissue engineering (47). Nanocomposite hydrogels are generally produced by chemically or physically cross-linking a polymer network to surface functional groups of the incorporated nanoparticles. Inorganic nanoparticles, such as silicate nanoparticles, magnetic nanoparticles and calcium phosphate nanocrystals (48-51), have been widely used to fabricate this type of hydrogel due to the abundance of surface functional groups that can interact with a polymeric network by covalent bonding, ionic interaction or hydrogen-bonding.

Studies of composite nanoparticle hydrogels have included specimens loaded with

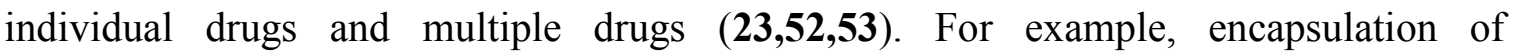
DNA/cationic polymer nanoparticles in hydrogel formed efficient surface-mediated delivery of genesinto cells (52). Lynch et al. (53) also fabricated a multiple drug loaded composite hydrogel by separately incorporating two different model drugs within hydrophobic microgels and anionic microgels and then embedding these within the host gel. Both model drugs showed simultaneous and independent release at different rates from the composite hydrogel. Most recently, Caicco et al. (54) developed a physically blended composite hydrogel of hyaluronan-methylcellulose (HAMC) for the localized 
delivery and sustainable release of CyclosporinA (CsA), a promising neuroprotective and neuroregenerative agent for neural stem/progenitor cells for treatment of stroke. The author incorporated CsA in HAMC gel by three different methods and compared their release rate from the gel. These incorporation types include a solubilized type (CsA/acetonitrile solution mixed with HAMC gel), a particulate type (CsA solid particulate dispersed in methyl cellulose solution and then embedded in HAMC gel) and poly(lactic-co-glycolic acid) (PLGA) microsphere-encapsulated CsA type (CsA loaded in PLGA microspheres and then embedded in HAMC gel).Interestingly, the in-vitro controlled release tests showed that solubilized CsA type directly released from HAMC gel only for 2 days, while the particulate type gave a controlled release over 7-10 days, but PLGA microsphere encapsulated CsA type showed sustainable release over 21 to 28 days. Most importantly, the CsA released from PLGA microspheres retained the same biological activity as fresh CsAwhen compared in aneurosphere assay (54).

Unfortunately, most of the investigations of polymeric nanoparticle composite hydrogel are still confined to use as drug delivery system. Due to the lack of effective surface functional groups or ligands, the polymeric nanoparticles themselves cannot self-assemble into well-organized arrays, leading to ineffective distribution within host polymer network via limited bond interaction, such as ionic interaction, or hydrogen bonding, or even just van der waals force. Although multiple drug loading and releasing in a hydrogel have been tried $(\mathbf{2 3 , 5 2})$, the nanoparticles tend to randomly aggregate leading to a non-uniform distribution of the drug and an uncontrolled concentration gradient of the drug within the scaffold if such techniques are used to fabricate composite hydrogel scaffolds for tissue regeneration. Thus the self-assembly of polymeric nanoparticles to form well-organized array with effective interaction with the host hydrogel scaffold are necessary to fabricate more sophisticated composite scaffold systems for tissue engineering. 


\subsubsection{Self-Assembled Peptide-Nanoparticle Precedents}

The key merit of using ion-complementary peptides to fabricate sophisticated assembly systems is their spontaneous self-assembly into stable $\beta$-sheets in aqueous solution, which occurs because of the strong electrostatic and selective interaction between opposite charges. Using peptide directed assembly of nanoparticles has received limited attention, and prior to this work was limited to inorganic nanoparticles (i.e. gold nanoparticles, nanotubes) arraysusing self-assembled peptide structures as templates or as assembly ligands (55-58). For example, Nikhil et al.(56) reported a well-organized one-dimensional gold nanoparticle line formed on a self-assembled polypeptide template, and the formation of the nanoparticle array was directed by positive charges on peptide fiber surface. Another recent report showed a simple method to fabricate a two-dimensional gold nanoparticle line pattern on mica surface from a designed $\beta$-sheet peptide (LELC16)-functionalized gold nanoparticles. Here the formation of gold nanoparticle alignment was directed by $\beta$-sheet self-assembly (57). Peptide amphiphiles with alkyl tails or polymer-peptide conjugates have also been studied, but still limited to nanofiber hydrogels for biological applications $(\mathbf{5 9 , 6 0 )}$. No precedent has been found for using peptide self-assembly to fabricate polymeric nanoparticle arrays or 3D scaffolds for biological application.

\subsubsection{Rationale and Advantages of Research Performed in Part A}

To address the limitations of the current scaffolding systems described above, it can be seen that a more advanced tissue scaffolding system can be achieved by using peptide-directed assembly of individual controlled-release devices (here core-shell nanoparticles) to give a new type of scaffold that combines the advantages of easy delivery of multiple drugs in matrices that give appropriate control of the release rate of each desired drug and can simultaneously serve as the scaffold device. We propose to fabricate a novel self-assembled polymeric nanoparticle fibers and 3D scaffolds using the attributes of 
ionic complementary $\beta$-sheet peptides, by functionalizing sets of nanoparticles with oppositely charged peptides, and then combining them so they self-assemble into fibers that are comprised of sequences of individual nanoparticles which can, if desired, carry active agents and control their release. The general mechanism is shown in Figure 1.1.
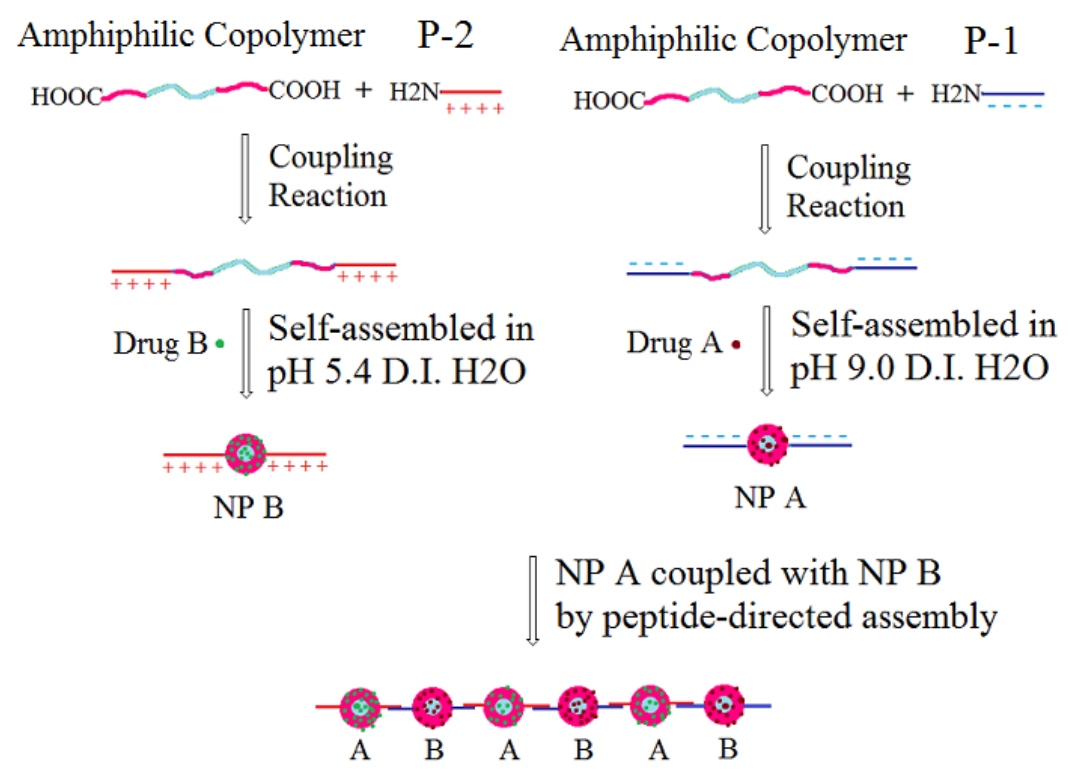

Figure 1.1. General procedure shows fabrication of polymeric nanoparticle fibers with multi-drug loading abilities.

As Figure 1.1 shows, the ends of two batches of amphiphilic copolymer are separately functionalized with the newly designed peptides (P1: $\mathrm{H}_{2} \mathrm{~N}-\mathrm{TTTT}$-AEAEAEAE-amide and P2: $\mathrm{H}_{2} \mathrm{~N}$-TTTT-AKAKAKAK-amide) to form peptide-copolymer conjugates, one carrying negative charges from $\mathrm{P} 1$, and the other carrying positive charges from P2. These two peptide-copolymer conjugates are then undergone two-level self-assembly in aqueous solution. The amphiphilic copolymer self-assembles into core-shell nanoparticles as drug carriers with charged peptide arms. The two batches of nanoparticles are coupled and further self-assembled into nanoparticle fibers directed by ionic complementary assembly between P1 and P2, and finally lead to 
form 3D nanoparticle scaffolds. Such self-assembled nanoparticle scaffolds possess several new features: (1) Simultaneously loading of different drugs; (2) Uniform distribution of different drugs in scaffolds; (3) Ability to give appropriate controlled release of each loaded drug from its own nanoparticle; (4) Flexibility and "injectability" of the self-assembled scaffolds; and (5) Further assembly with host peptide to form nanoparticle composite peptide hydrogel. Collectively these features allow both temporal and spatial control of the drug release and the surface properties of the scaffold, so the polymer nanoparticle surface can also be used as platform to further be modified or immobilized with functional groups or signals for specific tissue engineering application.

Here we use reversible addition fragmentation chain transfer (RAFT) polymerization to synthesize amphiphilic copolymer with reactive carboxylic end groups (HOOC-ABA-COOH) using S,S'-bis $\left(\alpha, \alpha^{\prime}\right.$-dimethylacetic acid) trithiocarbonate (BDAT) as the chain transfer agent (CTA) (61). RAFT is a controlled radical polymerization, which allows the synthesis of block copolymers with controlled block length and block sequence, and therefore, control over the size of both core and shell. The carboxylic acid end groups at both chain ends are required for the subsequent peptide coupling. The hydrophilic block for the nanoparticle shell is 1-vinyl-2-pyrrolidinone (VP), 2-hydroxyethyl methacrylate (HEMA) or 2-hydroxyethyl acrylate (HEA), while methyl methacrylate (MMA) is used for the hydrophobic core. These monomers were selected because VP, HEMA, HEA and MMA they have been widely used to synthesize biocompatible homopolymers or copolymers for biological applications (62-65), and because they are efficiently incorporated into polymer chains produced by RAFT polymerization (66).

Two ionic complementary peptides, $\mathrm{P} 1$ and $\mathrm{P} 2$, are synthesized by semi-automatic solid phase peptide synthesis strategy, using Fmoc chemistry, on rink amide resin. These detailed synthesis procedures are reported in Chapter 2 and Chapter 3. 
In Chapter 2 we will prove the hypothesis (Figure 1.1) that ionic complementary peptide-functionalized tri-block copolymer will self-assemble into nanoparticle fibers and further lead to 3D scaffolds, by functionalizing sets of amphiphilic copolymers synthesized by RAFT polymerization to form peptide-copolymer conjugates (P1-ABA-P1 and P2-ABA-P2, ABA: triblock copolymer) and perform self-assembly study in aqueous solution. Controlled release of a model drug (insulin) and a biocompatibility study of the synthesized materials using SW-620 cell lines will also be shown in this chapter.

In Chapter 3 we will continue to study the self-assembly behavior of the designed peptide-functionalized copolymer conjugates with different particle size and self-assembly with additional peptides (P1 and P2) to adjust the scaffold porosity and mechanical stability. Multiple drug loading capability and the ability of controlling the drug release rate from different nanoparticle compositions are also studied in this chapter, using several small molecules as model drugs: a hydrophobic model drug (4',5'-dibromofluorecein), a moderately hydrophobic model drug (nitrofurazone) and slightly water soluble hydrophilic model drug (amoxicillin). The biocompatibility test with fibroblast cells (NIH3T3 cell line) is also conducted by incubating the self-assembled 2D scaffold membrane with in a humidified incubator at $37{ }^{\circ} \mathrm{C}$ for 2 weeks, showing non-toxic and non-inhibition to the cell proliferation and migration. 


\subsection{Introduction for Part B}

The objective in Part $\mathrm{B}$ is to design and synthesize organic and inorganic biocide-loaded nanoparticles as controlled release systems for wood preservation. Using biocide-loaded nanoparticles as controlled release systems for wood preservation affords several advantages: (1) Environmentally friendly polymers can be used as biocide carriers; (2) Nanoparticles can effectively penetrate the wood interior to protect the wood interior as well as its exterior; (3) Nanoparticles can serve as a protective reservoir for the biocide and control the release rate of biocide to protect the treated wood from biological decay; (4) They can be prepared using simple and cost-effective routes. This section will introduce the background information, the rationale for the investigation, and the value of the work.

\subsubsection{Background of Wood Preservation}

Wood is an environmentally friendly and renewable material possessing unique physical and mechanical properties, including high strength, low thermal conductivity, the ability of damp sound or other vibrations, and has a pleasant appearance. Wood products have a long history of use as construction materials, but must be protected from biological attack by insects, fungi, mildew, etc. (67).

One of the most important wood preservatives, chromated copper arsenate (CCA) has been widely used in wood treatment for decades, but its use is being phased out due to environmental concerns over the arsenic. Two key advantages of CCA are that it "fixes" to wood, reducing its leaching from the wood into the environment, and CCA can be introduced into wood by pressure-treatment in aqueous solution. Any alternative to CCA must also be able to be introduced into wood in an aqueous medium so the wood surfaces are not oily, odor-free, and not toxic to non-target species.

Ideally CCA alternatives should not leach out of the wood, because this is inherently 
an inefficient use of expensive biocide, but also is toxic to species not attacking the wood that are not targets. The main alternatives to CCA are organic biocides, such as azoles, and arsenic-free cooper biocides. Organic biocides are not water-soluble and so may be introduced in aqueous emulsions. Copper-based preservatives are typically introduced as water-soluble salts, or in recent years as water-insoluble nanoparticulate copper salts (carbonates or oxides). The organic biocides and the water-soluble copper salts are known to leach from treated wood, especially in damp conditions such as when it rains or in sensitive wetland areas $(\mathbf{6 8 , 6 9 )}$. Prior to this work there were no published studies on leaching of copper oxide/carbonate nanoparticles from treated wood.

The research described in Part B of this dissertation investigated leaching of the organic biocide, tebuconazole, a commercial and widely used azole biocide, and copper oxide nanoparticles as a representative of the solid copper salt wood preservatives now in commercial use $\mathbf{( 7 0 , 7 1 )}$.

Tebuconazole is a water insoluble organic biocide, is susceptible to leaching, reducing the service life of the treated wood, and adding cost since more tebuconazole is used to compensate for that lost to leaching, as well as detrimental environmental effects to sensitive environments such as wetlands (72). Prior research in Dr. Heiden's lab showed wood treated with tebuconazole contained within controlled-release nanoparticles afforded protection to the treated wood, but did not study differences in leaching, and also the method used released the biocide more quickly than was desired (73-75). In Part B of this work the objective was to look at different matrix formulations, to allow a slower release rate to be obtained from the nanoparticles than was exhibited in the prior research, and to determine how effective the nanoparticle method was at reducing leaching when compared to wood treated with tebuconazole via a liquid-in-liquid emulsion.

Solid copper carbonate and oxide nanoparticles are also commercially employed for wood preservation, but leaching studies have not been published concerning these 
nanoparticles. The objective of the study using solid copper oxide nanoparticles was to determine if they leach as nanoparticles (a potential environmental concern), how their leaching compares with the soluble copper salts they compete against, and to determine if the size of the nanoparticle affects the leaching. Commercial copper salt nanoparticles are sold as "micronized" particles (76), but they contain a mixture of various sizes, most of which are sub-micron. Prior to this research no leaching studies on such particles-treated wood were in the literature.

\subsubsection{Rationale for Controlled Release of Organic Wood Preservatives}

In our earliest work on nanoparticle-treated wood preservation we used a precipitation route to prepare nanoparticles from pre-made random copolymers of poly(vinylpyridine-co-styrene) (73-75). The work was novel at that time, and showed effective penetration of the wood interior and protected the treated wood from decay. The method also had two major drawbacks: the release rate was controlled but faster than it needed to be, and the nanoparticle preparation method, while simple, required dilute $(\leq 1$ $\mathrm{wt} \%$ ) conditions. To address these drawbacks we then studied a preparation method that allowed us to synthesize core-shell nanoparticles in a one-pot/one-step nanoparticle. The critical advantages of this method are that the core, serving as a reservoir for the biocide, could be made as hydrophilic or hydrophobic as needed to give significant control over the rate to biocide release, while the shell could be made hydrophilic to stabilize the resulting nanoparticles in water. Furthermore, this method allows the polymer synthesis and the nanoparticle preparation to be made at $\geq 10 \mathrm{wt} \%$ solids in water. In that work gelatin was used to form the shell, and methyl methacrylate (non-polar) and hydroxyethyl methacrylate (polar) were grafted from the amine groups of the gelatin to prepare the amphiphilic copolymers that self-assembled into core-shell nanoparticles. When the polymerization was done in the presence of tebuconazole, the core-shell nanoparticles contained tebuconazole (77). However, while the gelatin is inexpensive and biologically safe, it is 
itself prone to fungal decay, and un-grafted gelatin complicated leaching tests. For that reason, in this work a similar study was done using chitosan, the second most abundant polysaccharide, which is also non-toxic, biodegradable, and bears numerous amine functional groups to be grafted with hydrophobic monomers to self-assemble into core-shell nanoparticle $(\mathbf{7 8 , 7 9 )}$. So we use chitosan as a starting material and grafted with methacrylate monomers such as methyl methacrylate (MMA) and hydroxyethyl methacrylate (HEMA) to form amphiphilic copolymers that self-assembled into solid tebuconazole-containing core-shell nanoparticles and treated wood with these nanoparticles to test and compare tebuconazole leaching. The reaction mechanism is outlined in Figure 1.2.

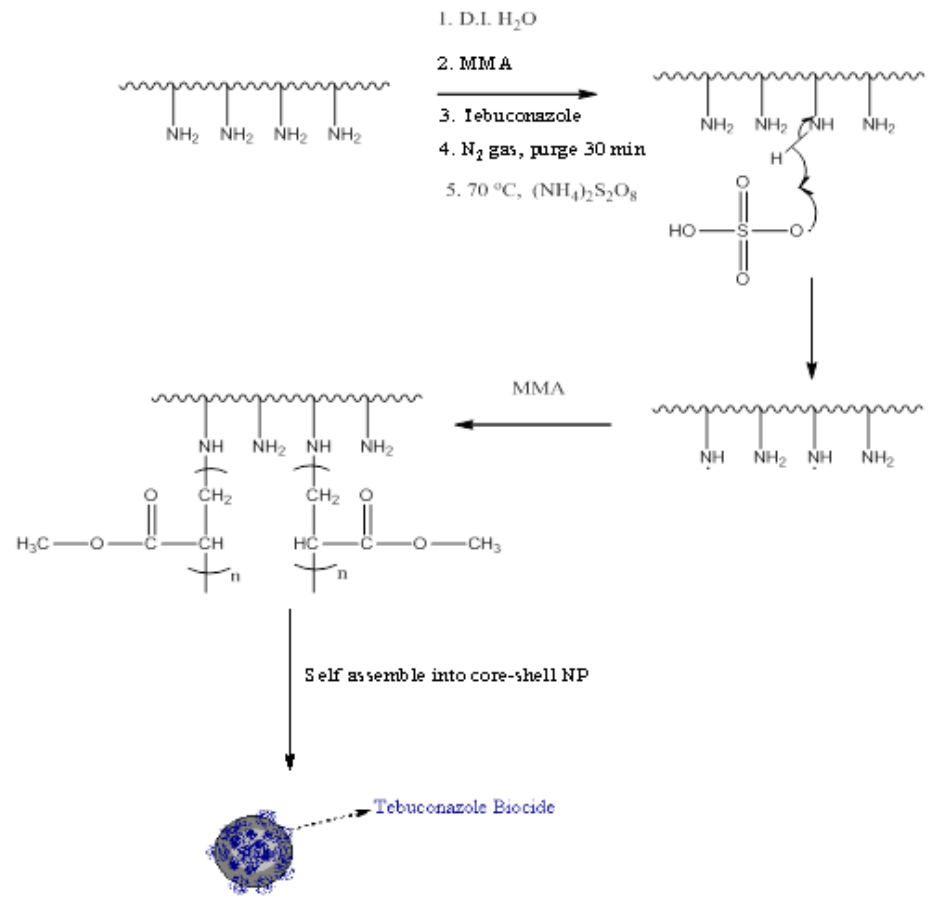

Figure 1.2. Mechanism of preparing self-assembled chitosan-g-PMMA nanoparticles loading with tebuconazole by one-pot method.

In Chapter 4, the synthesis and characterization of biocide-loaded chitosan-g-PMMA nanoparticles are shown, and the synthesis conditions and formulation 
are studied to determine how they affect nanoparticle size and biocide release rate. The nanoparticle size can be adjusted to $<150 \mathrm{~nm}$ by setting reaction concentration and chitosan to MMA ratio. The release rate of tebuconazole from nanoparticle can be manipulated by adjusting the monomer ratio of MMA:HEMA comprising the core during polymerization. Effective nanoparticle penetration throughout the treated wood, low leaching of biocide, and wood protection when tested for fungi decay are demonstrated in this chapter.

The biocide-loaded nanoparticle suspension prepared by such one-pot synthesis route is cost-effective and can be directly used to pressure treat wood products without post process. The reduced leaching of biocide will increase wood preservation period and eliminate toxic impact on neighboring environment, and low cost preparation method also matches the market requirement using nanotechnology for wood preservation.

\subsubsection{Rationale for Study of Copper Oxide Nanoparticle Leaching}

Since copper oxide nanoparticle treated wood products are already commercially available, but no research in the public domain has been conducted to determine if the nanoparticles are leached or factors affecting leaching behavior of copper from the treated wood including the size of nanoparticles. To answer these questions copper oxide nanoparticles were prepared at different diameters, with and without a polymer shell, and used to treat wood and test leaching. The reaction mechanism is shown in Figure 1.3. 


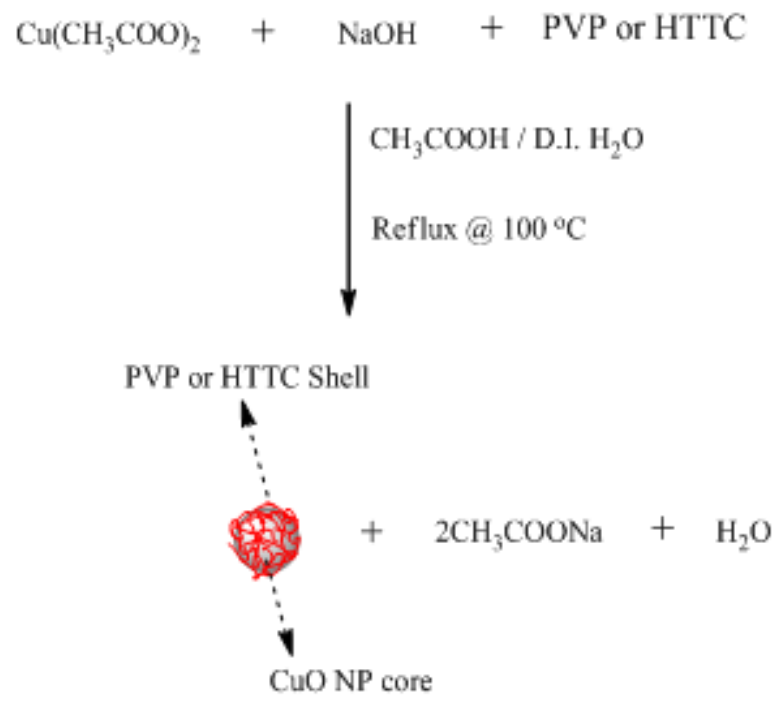

Figure 1.3. Synthesis of $\mathrm{CuO}$ nanoparticles with or without polymer-stabilizer by aqueous thermal hydrolysis using deionized water as the solvent and heating at reflux.

Chapter 5 describes how the copper oxide diameter was controlled so the leaching of nanoparticles with a uniform sized of $\sim 10 \mathrm{~nm}$ and $\sim 50 \mathrm{~nm}$, with and without a polymer shell. The questions this study asked and answered are: (1) Does nanoparticle size influence copper leaching; (2) Is any leached copper leached as nanoparticles; (3) Do copper oxide nanoparticles leach more or less than copper from to soluble copper salt (ACQ) used as a commercial wood preservative; (4) Do smaller nanoparticles show higher bioactivity compared to ACQ; and (5) Does a polymer shell on the copper oxide nanoparticles affect copper leaching? 


\section{References}

1. D. W. Hutmacher, Scaffolds in tissue engineering bone and cartilage. Biomaterials 2000, 21(24), 2529-2543.

2. Z. Zhang, J. Hu, P. X. Ma, Nanofiber-based delivery of bioactive agents and stem cells to bone sites. Advanced Drug Delivery Reviews 2012, 64, 1129-1141.

3. L.A. Smith, P.X. Ma, Nano-fibrous scaffolds for tissue engineering. Colloids and Surfaces B: Biointerfaces 2004, 39(3), 125-131.

4. R. R. Chen, D. J. Mooney, Polymeric growth factor delivery strategies for tissue engineering, Pharmaceutical Research 2003, 20 (8), 1103-1112.

5. M. J. Whitaker, R. A. Quirk, S. M. Howdle, K. M. Shakesheff, Growth factor release from tissue engineering scaffolds. Journal of Pharmacy and Pharmacology 2001, 53, $1427-1437$.

6. A. Schneider, J. A. Garlick, C. Egles, Self-assembling peptide nanofiber scaffolds accelerate wound healing. PLoS ONE, 2008, (1), e1410.

7. I.Pires, R. C. Bernardes, C. L. Lobo, M. A. Soares , J. G. Cunha-Vaz, Retinal thickness in eyes with mild nonproliferative retinopathy in patients with type 2 diabetes mellitus: Comparison of measurements obtained by retinal thickness analysis and optical coherence tomography. Arch. Ophthalmol. 2002,120,1301-1306.

8. R.G.Wylie, S. Ahsan, Y. Aizawa, K. L. Maxwell, C. M. Morshead, M. S. Shoichet, Spatially controlled simultaneous patterning of multiple growth factors in three-dimensional hydrogels. Nature Materials, 2011, 10, 799-806.

9. Jason A Burdick, Mariah N Mason, Adrian D Hinman, Kevin Thorne, Kristi S Anseth, Delivery of osteoinductive growth factors from degradable PEG hydrogels influences osteoblast differentiation and mineralization, Journal of Controlled Release, 2002, 83(1), 53-63. 
10. Niancao Chen, Zhaoyang Zhang, BoonchoySoontornworajit, Jing Zhou, Yong Wang, Cell adhesion on an artificial extracellular matrix using aptamer-functionalized PEG hydrogels, Biomaterials, 2012, 33(5), 1353-1362.

11. Sanabria-Delong N, Crosby AJ and Tew GN, Photo-cross -linked PLA-PEO-PLA hydrogels from self-assembled physical networks: mechanical properties and influence of assumed constitutive relationships, Biomacromolecules 2008, 9(10), 2784-2791.

12. Christine T. Schwall and Ipsita A. Banerjee, Micro- and Nanoscale Hydrogel Systems for Drug Delivery and Tissue Engineering, Materials 2009, 2, 577-612.

13. Steven M. Jay, Benjamin R. Shepherd,James P. Bertram, Jordan S. Pober and W. Mark Saltzman, Engineering of multifunctional gels integrating highly efficient growth factor delivery with endothelial cell transplantation, PNAS, 2010,107(10), 4669-4674.

14. Jenna M. Shapiro and Michelle L. Oyen, Hydrogel composite materials for tissue engineering scaffolds, JOM, 2013, 65(4), 505-516.

15. Silviya P. Zustiak, Yunqian Wei, and Jennie B. Leach. Protein-Hydrogel Interactions in Tissue Engineering: Mechanisms and Applications, Tissue Engineering Part B: Reviews. 2013, 19(2), 160-171.

16. Xiaoran Li, Zhifeng Xiao, Jin Han, Lei Chen, Hanshan Xiao, Fukai Ma, Xianglin Hou, Xing Li, Jie Sun, Wenyong Ding, Yannan Zhao, Bing Chen, Jianwu Dai, Promotion of neuronal differentiation of neural progenitor cells by using EGFR antibody functionalized collagen scaffolds for spinal cord injury repair, Biomaterials, 2013, 34(21), 5107-5116.

17. Samit K. Nandi, BiswanathKundu, DebabrataBasu, Protein growth factors loaded highly porous chitosan scaffold: A comparison of bone healing properties, Materials Science and Engineering: C, 2013, 33(3), 1267-1275. 
18. Marta Michalska, Katarzyna Kaplinska, Marek Mirowski, Andrzej Bodek, Kazimiera H. Bodek,Evaluation of the use of fibrin and microcrystalline chitosan membranes as carriers for transforming growth factor Beta-1, Journal of applied polymer science, $2013,127(5), 3506-3513$.

19. Ran Ito, Naoki Morimoto, LiemHieu Pham, TsuguyoshiTaira, Katsuya Kawai, and Shigehiko Suzuki, Efficacy of the controlled release of concentrated platelet lysate from a collagen/gelatin scaffold for Dermis-Like tissue regeneration, Tissue Engineering Part A. 2013, 19(11-12), 1398-1405.

20. Hongbin Zhang, Chen Zhao, Hui Cao, Guojie Wang, Li Song, GuoguangNiu, Huai Yang, Jie Ma and Siquan Zhu, Hyperbranched poly(amine-ester) based hydrogels for controlled multi-drug release in combination chemotherapy, Biomaterials, 2010, 31(20), 5445-5454.

21. Stefano Salmaso, Alessandra Semenzato, Sara Bersani, PietroMatricardi, Fabio Rossi and Paolo Caliceti, Cyclodextrin/PEG based hydrogels for multi-drug delivery, International Journal of Pharmaceutics, 2007, 345(1-2), 42-50.

22. Hang Thu Ta, Crispin R. Dass and Dave E. Dunstan, Injectable chitosan hydrogels for localized cancer therapy, Journal of Controlled Release, 2008, 126(3), 205-216.

23. Faming Chen, Min Zhang and Zhifen Wu, Toward delivery of multiple growth factors in tissue engineering, Biomaterials, 2010, 31(24), 6279-6308.

24. Shuguang Zhang, FabrizioGelain and Xiaojun Zhao, Designer self-assembling peptide nanofiber scaffolds for 3D tissue cell cultures, Seminars in Cancer Biology, $2005,15,413-420$.

25. Zhang S, Holmes TC, Lockshin C, Rich A. Spontaneous assembly of a self-complementary oligopeptide to form a stable macroscopic membrane. Proc. Natl. Acad. Sci. U. S. A. 1993, 90, 3334-38. 
26. Shuguang Zhang, FabrizioGelain and Xiaojun Zhao, Designer self-assembling peptide nanofiber scaffolds for 3D tissue cell cultures, Seminars in Cancer Biology, 2005, 15, 413-420.

27. Zhang S, Lockshin C, Cook R, Rich A. Unusually stable beta-sheet formation of an ionic self-complementary oligopeptide. Biopolymers, 1994, 34, 663-672.

28. Ramachandran S, Tseng Y, Yu B Y. Repeated rapid shear-responsiveness of peptide hydrogels with tunable shear modulus. Biomacromolecules, 2005, 6, 1316-1321.

29. Todd C. Holmes, Novel peptide-based biomaterial scaffolds for tissue engineering, TRENDS in Biotechnology, 2002, 20(1), 16-21.

30. FabrizioGelain, Akihiro Horii, Shuguang Zhang, Designer self-assembling peptide scaffolds for 3-D tissue cell cultures and regenerative medicine, Macromolecular Bioscience, 2007, 7(5), 544-551.

31. Nagai Y, Unsworth LD, Koutsopoulos S, Zhang S. Slow release of molecules in self assembling peptide nanofiber scaffold. J. Controlled Release 2006, 115, 18-25.

32. Koutsopoulos S, Unsworth LD, Nagai Y, Zhang S. Controlled release of functional proteins through designer self-assembling peptide nanofiber hydrogel scaffold. Proc. Natl. Acad. Sci. U. S. A. 2009, 106, 4623-8.

33. Horii A, Wang X, Gelain F, Zhang S. Biological designer self-assembling peptide nanofiber scaffolds significantly enhance osteoblast proliferation, differentiation and 3-D migration. PLoS One 2007, 2, e190.

34. Gelain F, Unsworth LD, Zhang S. Slow and sustained release of active cytokines from self-assembling peptide scaffolds. J. Controlled Release 2010, 145, 231-239.

35. Galler KM, Aulisa L, Regan KR, D'Souza RN, Hartgerink JD. Self-assembling multidomain peptide hydrogels: designed susceptibility to enzymatic cleavage allows enhanced cell migration and spreading. J. Am. Chem. Soc. 2010, 132, 3217-3223. 
36. Ying Zhao, Masayoshi Tanaka, Takatoshi Kinoshita, Masahiro Higuchi, Tianwei Tan, Self-assembling peptide nanofiber scaffolds for controlled release governed by gelator design and guest size, Journal of Controlled Release, 2010, 147(3), 392-399.

37. D. Roberts, C. Rochas, A. Saiani and A. F. Miller, Effect of peptide and guest charge on the structural, mechanical and release properties of $\beta$-sheet forming peptides, Langmuir, 2012, 28(46), 16196-16206.

38. Marco Biondi, Francesca Ungaro, FabianaQuaglia, Paolo Antonio Netti, Controlled drug delivery in tissue engineering. Advanced Drug Delivery Reviews 2008, 60(2), $229-242$.

39. C.X Song, V Labhasetwar, H Murhpy, X Qu, W.R Humphrey, R.J Shebuski, R.J Levy, Formulation and characterization of biodegradable nanoparticles for intravascular local drug delivery, Journal of Controlled Release, 1997, 43(2-3), $197-212$.

40. Petkar KC, ChavhanSS,Agatonovik-Kustrin S, Sawant KK. Nanostructured materials in drug and gene delivery: A review of the state of the art. Crit. Rev. Ther. Drug Carrier Syst. 2011, 28(2), 101-64.

41. Hunter C, Elsom J, Wibroe P, Moghimi M. Polymeric particulate technologies for oral drug delivery and targeting: a pathophysiological perspective. Nanomed-Nanotechnol, 2012, 8, S5-S20.

42. Miller T, Hill A, Uezguen S, Weigandt M, Goepferich A. Analysis of immediate stress mechanisms upon injection of polymeric micelles and related colloidal drug Carriers: implications on drug targeting. Biomacromolecules 2012, 13, 1707-1718.

43. Ding H, Wang X, Zhang S, Liu X. Applications of polymeric micelles with tumor targeted in chemotherapy. J. Nanopart. Res. 2012, 14, 1254 (P1-13). 
44. Khanbabaie R, Jahanshahi M. Revolutionary impact of nanodrug delivery on neuroscience. Curr. Neuropharmacol. 2012, 10(4), 370-92.

45. Omid C. Farokhzad and Robert Langer, Impact of nanotechnology on drug delivery, ACS Nano, 2009, 3(1), 16-20.

46. Vauthier C, Bouchemal K. Methods for the preparation and manufacture of polymeric nanoparticles. Pharm. Res. 2009, 26(5), 1025-1058.

47. Patrick Schexnailder and Gudrun Schmidt, Nanocomposite polymer hydrogels, Colloid PolymSci, 2009, 287, 1-11.

48. Hou Y, Matthews AR, Smitherman AM, Bulick AS, Hahn MS, Hou H, Han A, Grunlan MA Thermoresponsivenano-composite hydrogels with cell-releasing behavior. Biomaterials, 2008, 29 (22), 3175-3184.

49. Sunderland CJ, Steiert M, Talmadge JE, Derfus AM, Barry SE, Targeted nanoparticles for detecting and treating cancer, Drug Dev Res, 2006, 67(1), 70-93.

50. Leeuwenburgh SCG, Jansen JA, Mikos AG, Functionaliza-tion of oligo (poly(ethylene glycol) fumarate) hydrogelswith finely dispersed calcium phosphate nanocrystals for bone-substituting purposes. J BiomaterSci, Polym Ed, 2007, 18(12), $1547-1564$.

51. B. A. Rozenberg and R. Tenne, Polymer-assisted fabrication of nanoparticles and nanocomposites, Prog. Polym. Sci., 2008, 33, 40-112.

52. Pannier AK and Segura T., Surface- and hydrogel-mediated delivery of nucleic acid nanoparticles, Methods MolBiol, 2013, 948, 149-169.

53. Lynch I, de Gregorio P, Dawson KA, Simultaneous release of hydrophobic and cationic solutes from thin-film "plum-pudding" gels: a multifunctional platform for surface drug delivery, J PhysChem B., 2005, 109(13), 6257-6261.

54. Matthew J. Caicco, Michael J. Cooke, Yuanfei Wang, AnupTuladhar, Cindi M. Morshead, Molly S.Shoichet, A hydrogel composite system for sustained epi-cortical 
delivery of Cyclosporin A to the brain for treatment of stroke, Journal of Controlled Release, 2013, 166, 197-202.

55. Ryadnov MG, Cherny DI. Natively unfolded state for engineering nanoscalefibrillar arrays. Macromol. Biosci. 2012, 12, 195-201.

56. Sharma N, Top A, Kiick KL, Pochan DJ. One-dimensional gold nanoparticle arrays by electrostatically directed organization using polypeptide self-assembly. Angew. Chem. Int. Ed. 2009, 48, 7078-7082.

57. Tanaka M, Abiko S, Himeiwa T, Nakamura M, Koshikawa N, Kinoshita T. Two-dimensional self-assembly of amphiphilic peptide at the solid/water interface toward a facile method for metal nanoparticle alignment. Chem. Lett. 2012, 41, 1221-1222.

58. Shane Scanlon and AmaliaAggeli, Self-assembling pepide nanotubes, Nanotoday, 2008, 3(3-4), 22-30.

59. Nisbet DR, Williams RJ. Self-assembled peptides: Characterisation and in vivo response. Biointerphases 2012, 7(1-4), 2.

60. Webber MJ, Matson JB, Tamboli VK, Stupp SI. Controlled release of dexamethasone from peptidenanofiber gels to modulate inflammatory response. Biomaterials 2012, 33(28), 6823-6832.

61. Boyer C, Stenzel MH, Davis TP. Building nanostructures using RAFT polymerization. J. Polym. Sci. Pol. Chem. 2011, 49(3), 551-595.

62. Butruk B, Trzaskowski M, Ciach T. Fabrication of biocompatible hydrogel coatings for implantable medical devices using Fenton-type reaction. Mater. Sci. Eng., C 2012, 32, 1601-1609.

63. Guiseppi-Elie A, Dong C, Dinu CZ. Crosslink density of a biomimetic poly(HEMA)-based hydrogel influences growth and proliferation of attachment dependent RMS 13 cells. J. Mater. Chem. 2012, 22, 19529-19539. 
64. Lin $\mathrm{M}, \mathrm{Xu} \mathrm{P}$, Zhong $\mathrm{W}$, Preparation, characterization, and release behavior of aspirin-loaded poly(2-hydroxyethyl acrylate)/silica hydrogels. J. Biomed. Mater. Res. B- 2012, 100B(4), 1114-1120.

65. Li Z, Kawamura K, Kawashita M, Kudo T, Kanetaka H, Hiraoka M. In vitro assessment of poly(methylmethacrylate)-based bone cement containing magnetite nanoparticles for hyperthermia treatment of bone tumor, J. Biomed. Mater. Res. A 2012, 100A(10), 2537-2545.

66. Nicolas J, Mantovani G, Haddleton DM. Living radical polymerization as a tool for the synthesis of polymer-protein/peptide bioconjugates. Macromol. Rapid Commun. 2007, 28(10), 1083-1111.

67. A. Q. Nyrud, A. Roos, M. Rodbotten, Product attributes affecting consumer preference for residential deck materials, Can. J. Forest Res. 2008, 38, 1385-1396.

68. T. Townsend, H. Solo-Gabriele, T. Tolaymat, K. Stook and N. Hosein, Chromium, copper, and arsenic concentrations in soil underneath CCA-treated wood structures. Soil Sediment Contam., 2003, 12, 779-798.

69. S. Lebow, D. Foster and P. Lebow, Rate of CCA leaching from commercially treated decking, For. Prod. J., 2004, 54, 81-88.

70. Xiaochu Ding, Dana L. Richter, Laurent M. Matuanaand P.A. Heiden, Efficient one-pot synthesis and loading of self-assembled amphiphilic chitosan nanoparticles for low-leaching wood preservation, Carbohydrate Polymers, 2011, 86, 58-64.

71. Xiaochu Ding, Martha Barajas Meneses, Soha M. Albukhari, Dana L. Richter, Laurent M. Matuana, Patricia A. Heiden, Comparing leaching of different copper oxide nanoparticles and ammoniacal copper salt from wood, Macromolecular Materials and Engineering, 10 MAY 2013, DOI: 10.1002/mame.201200439.

72. Chelsea So-Ming Woo, Efficacy of tebuconazole and DDAC in shell-treated wood, University of British Columbia, 2006, Thesis for Master of Science. 
73. Liu, Y., Laks, P., and Heiden. P., Controlled Release of Biocides in Solid Wood. Part 1. Efficacy against Gloeophyllum trabeum, a Brown Rot Wood Decay Fungus. J. Appl. Polym. Sci. 2002, 86, 596.

74. Liu, Y., Laks, P., \&Heiden. P., Controlled Release of Biocides in Solid Wood. Part 2. Efficacy against Trametes versicolor and Gloeophyllum trabeum Wood Decay Fungi J. Appl. Polym. Sci. 2002, 86, 608.

75. Liu, Y., Laks, P., \&Heiden. P., Controlled Release of Biocides in Solid Wood; Part 3. Preparation and Characterization of Surfactant-Free Nanoparticles. J. Appl. Polym. Sci. $2002,86,615$.

76. M.H.Freeman, C.R. McIntyre, A comprehensive review of copper-based wood preservatives with a focus on new micronized or dispersed copper systems, Forest Products Journal 2008, 58, 6-27.

77. Salma, U., Chen, N., Richter, D.L.,Filson, P.B., Dawson-Andoh, B., Matuana, L., Heiden, P., Amphiphilic Core/Shell Nanoparticles to Reduce Biocide Leaching From Treated Wood, 1 - Leaching and Biological Efficacy. Macromolecular Materials and Engineering, 2010, 295(5), 442-450.

78. Aranaz, I., Harris, R., and Heras, A., Chitosan Amphiphilic Derivatives. Chemistry and Applications. Current Organic Chemistry, 2010, 14, 308-330.

79. Lao, B., Zhang, Z., Xu, H.H., Jiang, G., Novel amphiphilic chitosan derivatives: Synthesis, characterization and micellarsolubilization of rotenone. Carbohydrate Polymers, 2010, 82, 1136-1142. 
Studies of Novel Self-Assembly Structures from Peptide-Functionalized

Polymeric Micro- and Nanoparticles for Tissue Engineering

Xiaochu Ding*, Jagadeesh Janjanam, Ashutosh Tiwari, Martin Thompson, Patricia A.

Heiden*

Department of Chemistry, Michigan Technological University, Houghton, MI 49931, USA

The initial idea of this chapter was conceived by Dr. Heiden. All the design and research work were conducted by Mr. Xiaochu Ding, except cytotoxicity test on SW-620 cell lines was conducted by Dr. Jagadeesh Janjanam in Dr. Ashutosh Tiwari's lab. Dr. Martin Thompson taught X. Ding how to use the peptide synthesizer and assisted with the peptide synthesis.

Mr. Xiaochu Ding*

Department of Chemistry

Michigan Technological University

Dr. Ashutosh Tiwari

Department of Chemistry

Michigan Technological University

Dr. Patricia A. Heiden*

Department of Chemistry

Michigan Technological University
Dr. Jagadeesh Janjanam

Department of Chemistry

Michigan Technological University

Dr. Martin Thompson

Department of Chemistry

Michigan Technological University 


\section{Chapter 2}

Studies of Novel Self-Assembly Structures from Peptide-Functionalized

Polymeric Micro- and Nanoparticles for Tissue Engineering

Xiaochu Ding, JagadeeshJanjanam, AshutoshTiwari, Martin Thompson, Patricia A. Heiden*

Department of Chemistry, Michigan Technological University, Houghton, MI 49931, USA

*Corresponding author: +1 906487 3452; Fax: +1 9064872061.

Email address: paheiden@mtu.edu (P.A. Heiden).

This manuscript is in submission to the journal Macromolecular Bioscience. 


\begin{abstract}
A novel self-assembled 3D nanoparticle fiber scaffold was designed and synthesized with the ability to govern the controlled release of multiple drugs, by preparing ionic complementary peptide-functionalized amphiphilic block copolymers that self-assemble into polymeric nanoparticles, and then into fibers, leading to 3D fiber scaffolds. Two complementary peptide sequences were prepared that can assemble into $\beta$-sheets in aqueous solution (neutral water or PBS solution at $\mathrm{pH} 7.4$ ). The terminal amines of the peptides were coupled to amphiphilic triblock copolymers, synthesized by RAFT polymerization, with carboxylic acid terminals (HOOC-A-B-A-COOH), to give the desired peptide-copolymer conjugates. The peptides, copolymers, and peptide-copolymer conjugates were characterized by ${ }^{1} \mathrm{H}-\mathrm{NMR}$, matrix-assisted laser desorption/ionization time-of-flight mass spectrometry and FTIR. Optical microscopy confirmed the assembly of the peptide-copolymer conjugate nanoparticles into fibers and scaffolds. The assembly was not hindered when a protein (insulin) was included within the nanoparticles as an active ingredient. The release rate of insulin was measured over three weeks. MTS cytotoxicity tests on SW-620 cell lines showed that the peptides, copolymers and peptide-functionalized copolymers were biocompatible. The methodology of self-assembled nanoparticle fibers and 3D scaffolds combines the advantages of a flexible hydrogel scaffold and the versatility of controlled release systems.
\end{abstract}

Key words: $\beta$-sheet peptide, self-assembly, nanoparticle fiber, scaffold, RAFT polymerization, drug delivery, amphiphilic copolymer, core-shell 


\subsection{Introduction}

The self-assembly of $\beta$-sheet peptides is a well-established and versatile technique to fabricate 3-dimensional (3D) scaffolds for biological applications, with or without encapsulation of some bioactive agent (1). A typical $\beta$-sheet peptide motif is (AEAK) ${ }_{16}$-II (AEAEAKAKAEAEAKAK). This motif, composed of periodic repeats of ionic hydrophilic and hydrophobic amino acids, was first discovered in a yeast protein (2). This type of ionic complementary peptide shows a spontaneous self-assembly into stable $\beta$-sheets in aqueous conditions. The driving forces are the hydrophobic interactions of alanine (A) side chains and ion-pair interaction between negatively charged glutamic acid (E) side chains with positively charged lysine $(\mathrm{K})$ side chains. The strong ion-pair interaction leading to $\beta$-sheet formation was further illustrated using a pair of self-repulsing, but mutually attracting, peptide sequences that possessed positive charges (Ac-WKVKVKVKVK-amide) and negative charges (Ac-EWEVEVEVEV-amide) (3). On mixing this pair of oppositely charged peptides, a rapid assembly into a viscoelastic hydrogel occurred, even though the total peptide concentration was only $0.25 \mathrm{wt} . \%$. This hydrogel retained mechanical strength, even after repeated shear-induced breakdowns, due to the electrostatic interactions. The strong electrostatic and selective interaction between opposite charges demonstrated one of the key merits of using ion-complementary $\beta$-sheet motifs.

Those attributes are used in this work to provide a novel method to fabricate ordered nanoparticle arrays or fibers, by functionalizing sets of nanoparticles with oppositely charged peptides, and then combining them so they self-assemble into fibers that are themselves comprised of sequences of individual nanoparticles which can, if desired, be used to carry active agents and control their release. This is the hypothesis we are going to prove in the present work, in its simplest form, by assembling the fibers and scaffolds with no more than two nanoparticles and a single active agent. 
The use of a single drug at this early stage of the research is to minimize the number of variable that might affect the assembly. However, it is also important to prove the ability to control the release of an active agent, because in many biological applications, it is desirable for the scaffold to contain and potentially release various "active agents" (e.g. low molecular weight drugs or various proteins), to direct cell activities or improve therapeutic efficacy. Here, for the sake of simplicity we will use the term drug to refer to any active agent.

Peptide hydrogels have been a popular device for use as both a controlled drug delivery system and 3D scaffold, this is typically done by simply physically entrapping a drug into the peptide scaffold during mixing before gel formation $(\mathbf{4 , 5})$, or chemically bonded onto the $\mathrm{C}$-terminal or $\mathrm{N}$-terminal (6), or some combination of both methods to load multiple active agents $\mathbf{( 7 , 8 )}$. However, these methods have significant limitations with respect to the quantity of drug(s) that can be "loaded", and the drug(s) may have an impact on the subsequent self-assembly process or the mechanical stability of the formed hydrogel, and there is very limited ability to control the release of the drug(s). Therefore, the hydrogel itself must be designed in conjunction with the specific $\operatorname{drug}(\mathrm{s})$ that will be used. Moreover, effective distribution of drugs in scaffolds is not always easy to achieve. Controlled release nanoparticles can potentially be distributed within hydrogels, but these also are sometimes difficult to distribute uniformly or effectively, and also might interfere with assembly.

Because of these limitations, a more powerful and versatile technique is needed that can allow multiple drugs to be incorporated within scaffolding and also allows the release rate of each drug to be controlled appropriately. There are many ways to deliver drugs, such asmicelles, colloids, capsules, and solid nanoparticles, including self-assembled core-shell nanoparticles (9). Solid nanoparticles have been used for oral drug delivery (10), 
epidermal injection (11), targeted delivery of anticancer drugs to cancer cells (12), and to deliver drugs for central nervous system regeneration (13).

Core-shell polymeric nanoparticles are particularly attractive for use in tissue scaffolding because the core can potentially be designed to be suitable for a desired hydrophilic or hydrophobic drug while the shell can be designed to be suitable for the use environment. Also, core-shell nanoparticles are relatively easy to prepare with a controlled particle size and various bio-compatible hydrophilic and hydrophobic monomers are commrecially available to make self-assembling block copolymers that give core-shell nanostructures (14).

Thus, we propose a new and highly versatile type of self-assembly scaffolding system that combines controlled release technology with core-shell self-assembly techniques and peptide self-assembling techniques, to form "particle fibers". The particles themselves can potentially be of any size or composition, and synthesized by any technique, but in this work we opted for amphiphilic tri-block copolymers (HOOC-ABA-COOH) synthesized by RAFT polymerization, using BDAT as the CTA (15). RAFT is a controlled radical polymerization, which allows the synthesis of block copolymers with controlled block length and block sequence, and therefore, control over the size of both core and shell, and we used BDAT as the CTA to give carboxylic acid end groups at both chain ends, which are required for the subsequent peptide coupling. The hydrophilic block for the nanoparticle shell was 1-vinyl-2-pyrrolidinone (VP), 2-hydroxyethyl methacrylate (HEMA) or 2-hydroxyethyl acrylate (HEA), while methyl methacrylate (MMA) was used for the hydrophobic core. These monomers were selected because VP, HEMA, HEA and MMA they have been widely used to synthesize biocompatible homopolymers or copolymers for biological applications (16-19), and because they are efficiently incorporated into polymer chains produced by RAFT polymerization (20). 
The polymeric micro- or nanoparticles (or combination of both), that may or may not contain drugs, as desired, are functionalized with oppositely charged $\beta$-sheet peptides. Peptide directed assembly of nanoparticles has received some attention, but so far it has been limited to inorganic nanoparticle arrays using self-assembled peptide structures as templates (21-23). For example, Nikhil et al. reported a well-organized one-dimensional gold nanoparticle line formed on self-assembled polypeptide template, and the formation of the nanoparticle array was directed by regulatory-presented positive charges on peptide fiber surface (22). Another recent report showed a simple method to fabricate a two-dimensional gold nanoparticle line pattern on mica surface from a designed $\beta$-sheet peptide (LELC16)-functionalized gold nanoparticles. Here the formation of gold nanoparticle alignment was directed by $\beta$-sheet self-assembly (23). Peptide amphiphiles with alkyl tails or polymer-peptide conjugates have also been widely studied, but still limited to nanofiber hydrogels for biological applications $(\mathbf{2 4 , 2 5})$.

This research appears to be the first to report the self-assembly of polymeric nanoparticles into fiber and scaffolds. This is accomplished by using $\beta$-sheet peptides to direct the assembly process, by designing and synthesized two mutually attractive peptide sequences, peptide1 (P1: $\left.\mathrm{H}_{2} \mathrm{~N}-\mathrm{TTTT}-\mathrm{AEAEAEAE}-\mathrm{amide}\right)$ and peptide2 (P2: $\mathrm{H}_{2} \mathrm{~N}$-TTTT-AKAKAKAK-amide). These complementary peptides carry opposite charges, and by coupling them to the carboxylic acid terminals of these amphiphilic tri-block copolymers (HOOC-ABA-COOH), to form the peptide-copolymer conjugates P1-ABA-P1 and P2-ABA-P2 respectively.

The peptide-copolymer conjugates undergo multiple levels of self-assembly in aqueous solution. First, the amphiphilic copolymers can self-assemble to give nanoparticles with hydrophobic cores and hydrophilic shells, while the peptide-functionalities assemble the nanoparticles into fibers, and then further assemble $\beta$-sheets to form 3D nanoparticle scaffolds. To the best of our knowledge, this polymeric 
nanoparticle fibers and 3D scaffolds are a fundamentally novel self-assembly system because these systems allow multiple active agents to be introduced into the assembled structure using a composition suitable for each active ingredient, and giving an unprecedented level of both temporal and spatial control over the structure of the scaffold and the release of the active agents. In this work we report on the design and synthesis of the scaffold and demonstrate the capability of controlled drug release (insulin as a model protein) and suitability as a versatile tissue scaffold. 


\subsection{Materials and methods}

\subsubsection{Materials}

Fmoc amide rink resin (0.65 $\mathrm{mmol} / \mathrm{g})$, Fmoc amino acids, and 2-(7-aza-1-H-benzotriazol-1-yl)-1,1,3,3-tetramethylaminium (HATU, coupling agent), were purchased from AAPPTec company (Louisville, KY). N,N-Dimethylformamide (DMF, $\geq 99.9 \%$ ), N,N-diisopropylethylamine (DIPEA,99.5\%), piperidine (PIP, 99.5\%) and cleaving agents, including trifluoroacetic acid (TFA, 99\%), thioanisole (99\%) and anisole $(99 \%)$ were purchased from Sigma-Aldrich. All reagents for peptide synthesis were used as received. 1-Vinyl-2-pyrrolidinone (VP) ( $\geq 99 \%)$, 2-hydroxyethyl acrylate (HEA) (96\%), 2-hydroxyethyl methacrylate (HEMA) (97\%), methyl methacrylate (MMA) (99\%), 1,4-dioxane (99+\%), hexane (98.5\%), 2,2'-azobisisobutyronitrile (AIBN) (98\%), and phosphate buffered saline (biotech) were purchased from Sigma-Aldrich. 1,2-Ethanedithiol (98.0\%) was from Fluka, ethyl alcohol (200 proof, anhydrous) was from PHARMCO-AAPER, and diethyl ether (anhydrous) was from Mallinckrodt Baker Inc. (Phillipsburg, USA). The monomers HEA, HEMA and VP were purified before use by passing through a neutral alumina column. MMA was distilled before use. All other reagents were used as received. SW-620 cell was purchased from ATCC and CellTiter 96 AQueous Non-Radioactive Cell proliferation Assay (MTS) kit was from Promega Corp.

\subsubsection{Synthesis of peptides (P1 and P2) bearing opposite charges}

Peptides P1 (H ${ }_{2} \mathrm{~N}-\mathrm{Thr}$-Thr-Thr-Thr-Ala-Glu-Ala-Glu-Ala-Glu-Ala-Glu-amide) and P2 ( $\mathrm{H}_{2} \mathrm{~N}$-Thr-Thr-Thr-Thr-Ala-Lys-Ala-Lys-Ala-Lys-Ala-Lys-amide) were synthesized on rink amide resin (1.0 g, $0.65 \mathrm{mmol}$ amine/g per batch) by semi-automated solid phase peptide synthesis (Endeavor 90I, AAPPTec LLC, Louisville, KY, USA), using Fmoc chemistry. The side chains of threonine, lysine and glutamic acid were protected with tert-Butyl (tBu), t-Butyloxycarbonyl (Boc) and O-tert-Butyl (OtBu) groups, respectively.After swelling and washing the resin beads in DMF, the resin was 
deprotected 2 times with a 20\% PIP/DMF solution $(15 \mathrm{~mL}$ each time) at room temperature ( 5 min the first time and 15 min the second time) with washing of DMF to remove the Fmoc protection group from the resin. Then the first amino acid $(4 \times 0.65$ mmol) was dissolved in DIPEA/DMF $(5.2 \mathrm{~mL}, 0.5 \mathrm{M})$ in the presence of HATU $(4 \times 0.65$ $\mathrm{mmol}$ ), and transferred into a reaction vessel to attach to the resin. After de-protecting the Fmoc protecting group from the previously attached amino acid, the remaining amino acids (each 4x0.65 mmol) were similarly dissolved in DIPEA/DMF in the presence of HATU and attached step-wise to the resin beads according to the designed sequence. After finishing the coupling reactions of the desired 12 amino acids, the peptide-bound resin beads were transferred into a reaction flask and treated with a cocktail of cleavage reagents, TFA/Thioanisole/Ethane dithiol/Anisole $(90: 5: 3: 2 \mathrm{v} / \mathrm{v})$, to cleave the peptides from the resin beads. The cleaved peptide/cocktail solution was isolated from the solid resin beads by filtering through a glass column filled with glass wool, followed by precipitation into cold diethyl ether, placed in an ice/ethanol bath, to collect the synthesized peptide solids. The peptide solids were washed 3 times with cold diethyl ether and freeze-dried for storage. Scheme 2.1 shows the P1 and P2 design and the solid phase peptide synthesis. 

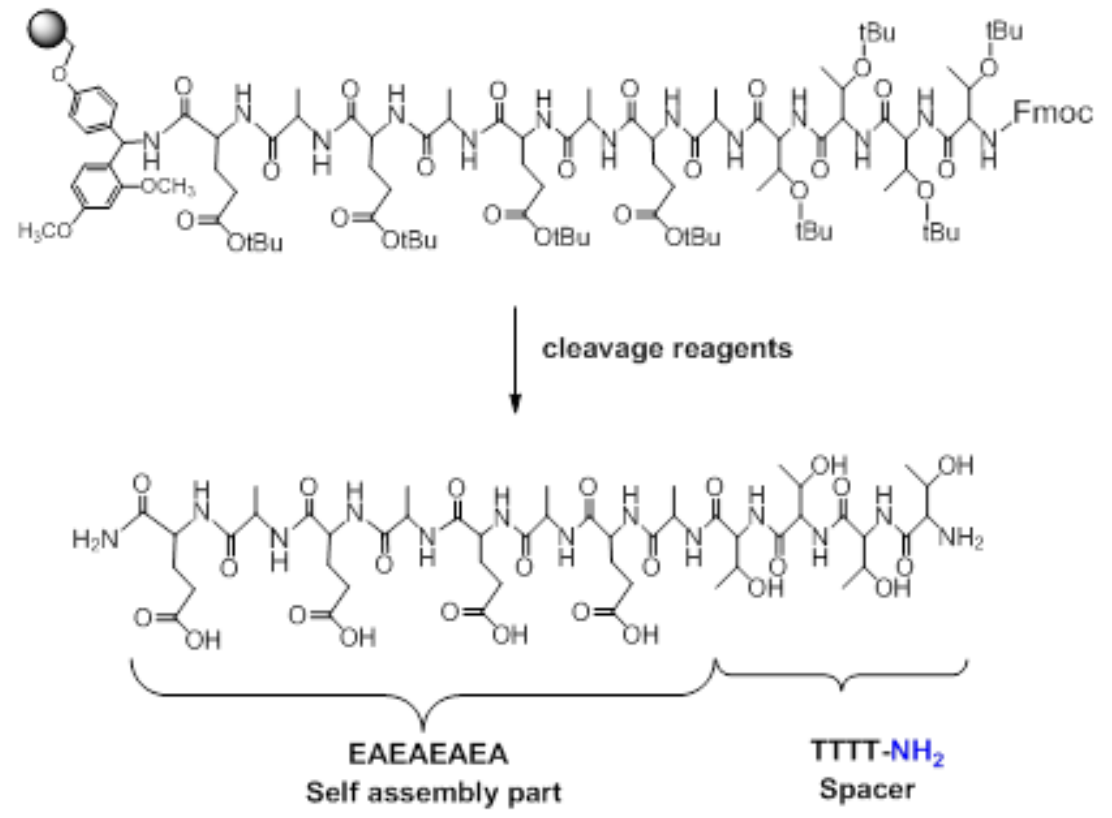

(a)
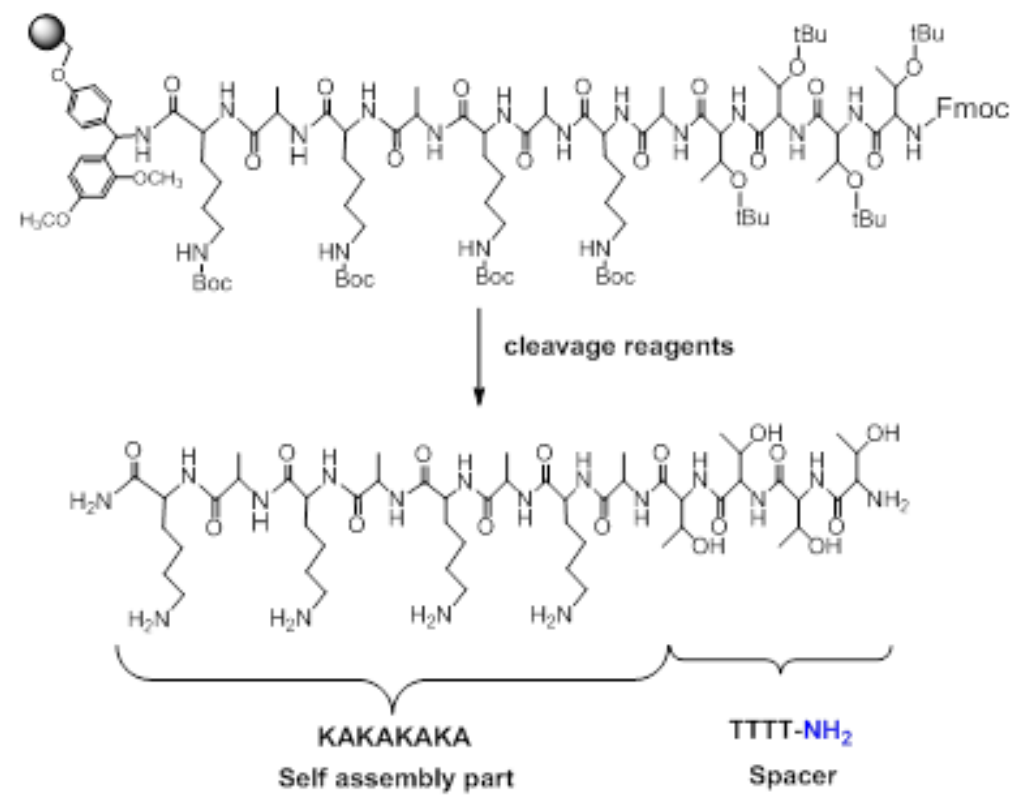

(b)

Scheme 2.1. The design and synthesis of (a) P1 and (b) P2, by solid phase peptide synthesis using Fmoc rink amide resin.

After synthesis and isolation, P1 and P2 were analyzed by MALDI-TOF MS (Microflex LRF, Bruker Daltonics, Billerica, USA). Briefly, P1 and P2 were dissolved in 
a matrix solution (50:50 water/acetonitrile with $0.1 \%$ TFA) to form two solutions, each with a concentration of $\sim 100 \mathrm{pmol} / \mu \mathrm{L}$. Separately, $20 \mathrm{mg}$ of $\alpha$-cyano-4-hydroxycinnamic acid (the matrix compound) was also dissolved in $1 \mathrm{~mL}$ of the described matrix solution, and the peptide and matrix solutions were then combined at a ratio of 1:10 (v/v). Then 2 $\mu \mathrm{L}$ of each combined solution were placed on a ground steel plate, then dried, and the ground steel plate was set up for MALDI-TOF MS analysis using a suitable analysis model (LP_pepmix model, close to the theoretical molecular weight of the peptides). By MALDI-TOF the molecular weights of P1 and P2 were found to be $1246.42[\mathrm{M}(\mathrm{P} 1)+$ $\left.\mathrm{Na}^{+}\right]$and $1220.01\left[\mathrm{M}(\mathrm{P} 2)+\mathrm{H}^{+}\right]$Da, which are in good agreement with the theoretical molecular weights of 1223.25 and 1219.45 Da respectively (Figure 2.1S a,b). ${ }^{1} \mathrm{H}-\mathrm{NMR}$ (Varian Unity INOVA $400 \mathrm{MHz}$, McKinley Scientific, Sparta, NJ, USA) confirmed the peptide structure, with the peaks were assigned as follows.

P1 (DMSO-d6, 8): 1.05-1.09 (9H, $-\underline{\mathrm{C}}_{3}$ from Thr), 1.22 (12H, $-\underline{\mathrm{CH}}_{3}$ from Ala), 1.77-1.89 (8H, $-\mathrm{C}_{2}-$ from Glu $), 2.26\left(8 \mathrm{H},-\underline{\mathrm{CH}}_{2}-\mathrm{C}=\mathrm{O}\right.$ from $\left.\mathrm{Glu}\right), 3.77(3 \mathrm{H}, \mathrm{O}-\mathrm{C} \underline{\mathrm{H}}-\mathrm{C}$

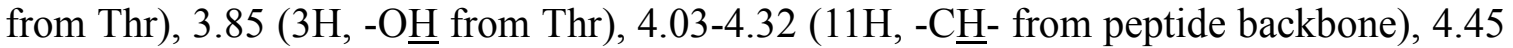
$\left(2 \mathrm{H}, \mathrm{C}-\mathrm{N}_{2}\right.$ from end amine), $7.04\left(2 \mathrm{H}, \mathrm{O}=\mathrm{C}-\mathrm{N}_{2}\right.$ from terminal amide), 7.70-8.06

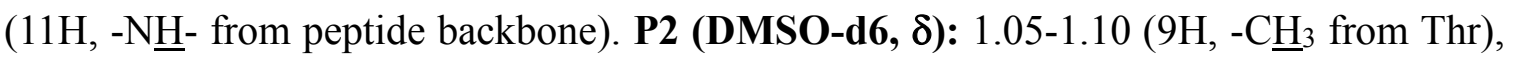
1.16-1.22 (8H, $\gamma-\underline{\mathrm{C}}_{2}$ - from Lys), 1.32 (8H, $\sigma-\mathrm{C}_{2}-$ from Lys), 1.51 (12H, $-\mathrm{C}_{3}$ from Ala), 1.65 (8H, $\beta$ - $\underline{\mathrm{C}}_{2}$-from Lys), 2.75 (8H, $-\underline{\mathrm{C}}_{2}-\mathrm{N}$ from Lys), 3.78-3.86 (11H, - $\underline{\mathrm{H}}_{2}$

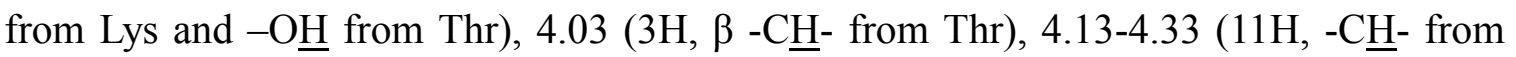
peptide backbone), 4.43(2H, $-\mathrm{N}_{2}$ from terminal amine), $7.03\left(2 \mathrm{H}, \mathrm{O}=\mathrm{C}-\mathrm{N}_{2}\right.$ from

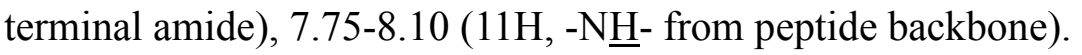

\subsubsection{Synthesis of $S, S^{\prime}$-bis $\left(\alpha, \alpha^{\prime}\right.$-dimethylacetic acid) trithiocarbonate (BDAT)}

The RAFT chain transfer agent, BDAT, was synthesized and purified according to a reported method (26). The detailed procedure is provided in supplementary information. The chemical structure of the purified BDAT was confirmed by ${ }^{1} \mathrm{H}-\mathrm{NMR}\left(\mathrm{CD}_{3} \mathrm{CL}\right):-\mathrm{CH}_{3}$ 
$(\mathrm{s}, 12 \mathrm{H})$ at $1.67 \mathrm{ppm}$ and $-\mathrm{COOH}(\mathrm{s}, 2 \mathrm{H})$ at $13.0 \mathrm{ppm}$. The chemical reaction is shown in Scheme 2.2.

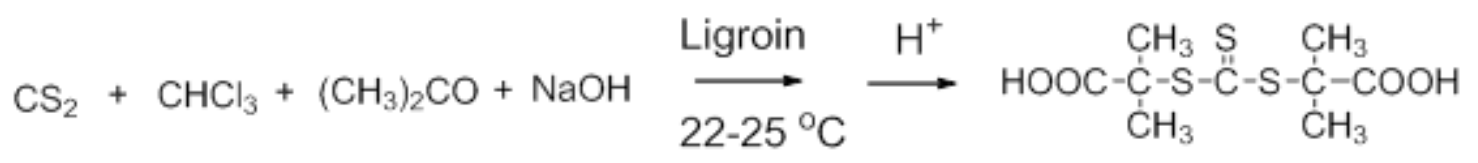

Scheme 2.2. Synthesis of BDAT.

\subsubsection{Synthesis of hydrophilic block (Macro-CTA) and amphiphilic tri-block copolymer using BDAT as RAFT CTA}

The amphiphilic tri-block copolymers PVP-b-PMMA-b-PVP, PHEMA-b-PMMA-b-PHEMA, and PHEA-b-PMMA-b-PHEA, terminated with carboxylic acid groups, were synthesized in two steps. First, the hydrophilic block (PVP, PHEMA or PHEA) was synthesized by RAFT polymerization using BDAT as CTA, AIBN as the initiator, and the desired hydrophilic monomer, at ratios of $[\mathrm{M}]_{\mathrm{o}}:[\mathrm{CTA}]_{\mathrm{o}}:[\mathrm{I}]_{\mathrm{o}}=1000: 5: 1$. The typical synthesis was done beginning with monomer $(30$ mmol),BDAT $(0.15 \mathrm{mmol})$ and AIBN $(0.030 \mathrm{mmol})$ in a solution of 1,4-dioxane $(16 \mathrm{~mL})$ in a $50 \mathrm{~mL}$ three neck round bottom flask. The reaction solution was first purged by nitrogen gas for $0.5 \mathrm{~h}$, and then heated to $70{ }^{\circ} \mathrm{C}$. The reaction was maintained at that temperature under nitrogen atmosphere for $24 \mathrm{~h}$. The product was collected by precipitation in cool diethyl ether, and the solvation/precipitation step was repeated 3 times to obtain the purified polymers. In the second step, the purified hydrophilic polymer was used as a macro-CTA to synthesize the amphiphilic tri-block copolymer. The ratio of reactants used was MMA:macro-CTA: AIBN of 4.8:0.4:0.0012 g/g (48000 : 45 : 1, mol:mol). These were dissolved together into $18 \mathrm{~mL}$ of a mixed solvent of 1,4-dioxane/DMF $(2: 1, \mathrm{v} / \mathrm{v})$ in a $50 \mathrm{~mL}$ three neck round bottom flask. The reaction 
solution was purged with nitrogen gas for $0.5 \mathrm{~h}$ and then reacted at $70{ }^{\circ} \mathrm{C}$ for $48 \mathrm{~h}$ under nitrogen atmosphere. The polymer was collected by solvating and precipitating in cool ethanol/acetone $(7: 1, \mathrm{v} / \mathrm{v}) 3$ times to obtain the purified amphiphilic tri-block copolymer. The feedstock ratios and yield \% of (co)polymers are given in Table2.1.

Scheme 2.3 shows the synthetic route to prepare the hydrophilic block (macro-CTA) and amphiphilic copolymer with carboxylic acid terminals.

Table 2.1. Summary of feedstock, $M_{N}$ and yield (\%) from the RAFT synthesis of the hydrophilic macro-CTA and tri-block copolymers.

\begin{tabular}{ccccc}
\hline Macro-CTA & & & & Yield \\
& {$[\mathrm{M}]_{\mathrm{o}}:[\mathrm{CTA}]_{\mathrm{o}}:[\mathrm{I}]_{\mathrm{o}}$} & $\mathrm{X}_{\mathrm{N}}^{\mathrm{a}}$ & $\mathrm{M}_{\mathrm{N}}^{\mathrm{a}}, \mathrm{Da}$ & $\%$ \\
\hline PVP & $1000: 5: 1$ & 78 & 8954 & 84.0 \\
PHEMA & $1000: 5: 1$ & 66 & 8981 & 97.4 \\
\hline PHEA & $1000: 5: 1$ & 74 & 8966 & 97.7 \\
\hline & Mass Ratio & & & \\
\hline Copolymer & (MMA): & & & Yield \\
& (Macro-CTA ):AIBN & & & $\%$ \\
\hline PVP-b-12PMMA-b-PVP & $12: 1: 0.0012$ & $78 / 382$ & 47200 & 53.1 \\
\hline PHEMA-b-12PMMA-b-PHEMA & $12: 1: 0.0012$ & $66 / 726$ & 81668 & 53.3 \\
PHEA-b-12PMMA-b-PHEA & $12: 1: 0.0012$ & $74 / 644$ & 73443 & 49.5 \\
\hline
\end{tabular}

a. $\mathrm{M}_{\mathrm{N}}$ by MALDI-TOF; $\mathrm{X}_{\mathrm{N}}$ units calculated as $X_{N}=\frac{M_{N}-M_{B D A T}}{M_{\text {Monomer }}}$.

b. $\mathrm{M}_{\mathrm{N}}$ calculated from ${ }^{1} \mathrm{H}$ NMR by ratio of integrated peaks. 

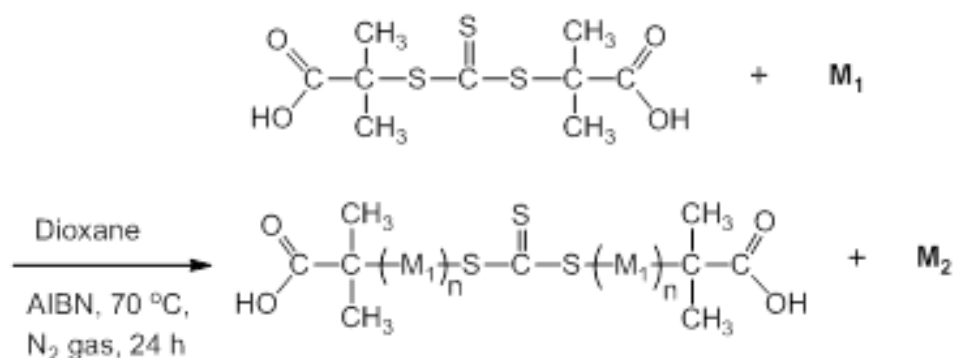

(a)

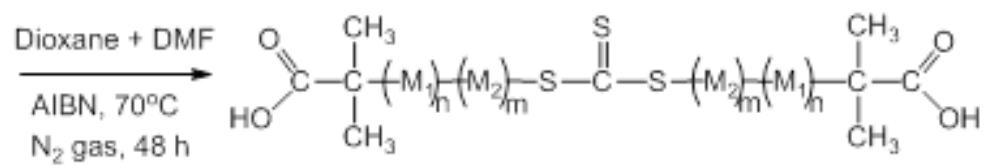

(b)

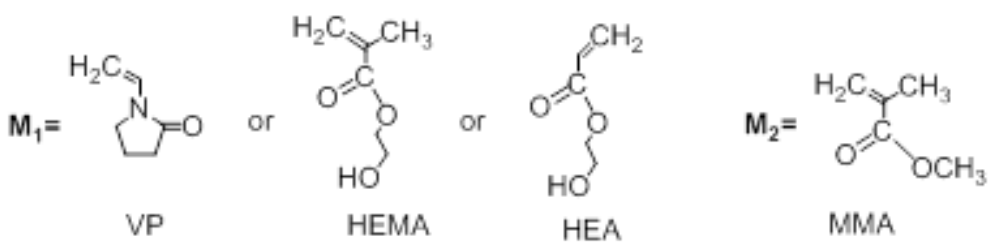

Scheme 2.3. Synthesis of (a) hydrophilic block macro-CTA and (b) the amphiphilic triblock copolymers, by RAFT polymerization using BDAT as CTA.

\subsubsection{Characterization of hydrophilic blocks and tri-block copolymers}

The molar mass of the hydrophilic block was determined from MALDI-TOF MS analysis using DMF as solvent and 2,5-dihydroxybenzoic acid (DHB) as the matrix compound. The polymer solution $(\sim 2 \mathrm{mg} / \mathrm{mL}$ in DMF) and a DHB solution $(20 \mathrm{mg} / \mathrm{mL}$ in DMF) were prepared separately, and then the polymer solution was added into the DHB solution at a ratio of $1: 10(\mathrm{v} / \mathrm{v})$. Then $2 \mu \mathrm{L}$ of the combined solution was dripped onto a ground steel plate and allowed to air dry over $48 \mathrm{~h}$. The sample plate was analyzed by MALDI-TOF MS (LP_14 KDa). The molecular weight $\left(\mathrm{M}_{\mathrm{N}}\right)$ and repeat units $\left(\mathrm{X}_{\mathrm{N}}\right)$ (calculated from $\mathrm{M}_{\mathrm{N}}$ ) of hydrophilic block are summarized in Table 2.1.

The amphiphilic copolymer was analyzed by ${ }^{1} \mathrm{H}-\mathrm{NMR}$, and the molecular weight $\left(\mathrm{M}_{\mathrm{N}}\right)$ was calculated by integrating the peak areas associated with the blocks. For 
example, PVP-b-12PMMA-b-PVP shows the peak area of $\delta: 3.34\left(-\mathrm{CH}_{2}-\mathrm{N}\right.$, from PVP block) and peak area of $\delta: 3.53\left(-\mathrm{OCH}_{3}\right.$ from PMMA block, - $\mathrm{CH}$ - from PVP block) are used to calculate the block ratio of $\mathrm{X}_{\mathrm{N}}(\mathrm{PVP}): \mathrm{X}_{\mathrm{M}}(\mathrm{PMMA})=1: 4.9$ based on the below equation.

$$
\frac{2 X n}{3 X m+X n}=\frac{\operatorname{Area}_{\left(-\mathrm{CH}_{2}-\mathrm{N}\right)}}{\text { Area }_{\left(-\mathrm{OCH}_{3},-\mathrm{CH}-\right)}}=\frac{4.49}{35.17}
$$

The block ratio $\left(\mathrm{X}_{\mathrm{N}}: \mathrm{X}_{\mathrm{M}}\right)$ of PHEMA-b-12PMMA-b-PHEMA and PHEA-b-12PMMA-b-PHEA were calculated in a similar manner from the ${ }^{1} \mathrm{H}-\mathrm{NMR}$ spectra (Figure 2.2S a,b and Figure 2.3S a,b, with calculation equation) and found to be 1:11 and 1:8.7 respectively. From these data the $X_{N}$ of the hydrophilic blocks and $X_{M}$ of the hydrophobic PMMA block for all three copolymers were found to be as follows: $(\mathrm{PVP})_{39}$-b-(PMMA) $)_{32}-\mathrm{b}-(\mathrm{PVP})_{39}, \quad$ (PHEMA) $)_{33}-\mathrm{b}-(\mathrm{PMMA})_{726}-\mathrm{b}-(\mathrm{PHEMA})_{33}$ and

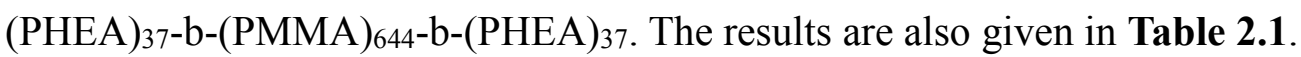

${ }^{1} \mathrm{H}-\mathrm{NMR}$ peak assignments for hydrophilic blocks and copolymers are given below.

PVP Block (in $\left.\mathbf{D}_{2} \mathbf{O}, \delta\right)$ : 1.46-1.61(- $\underline{\mathrm{C}}_{2}-$ from backbone), 1.90 (- $\underline{\mathrm{C}}_{2}-$ from vinylpyrrolidone ring), 2.18-2.32 (- $\underline{\mathrm{C}}_{2}-\mathrm{C}=\mathrm{O}$ from vinylpyrrolidone ring), $3.19\left(-\mathrm{C}_{2}-\mathrm{N}-\right.$ from vinylpyrrolidone ring), 3.52-3.66 (- $\underline{\mathrm{C}}-$ - from backbone). PHEMA Block (in

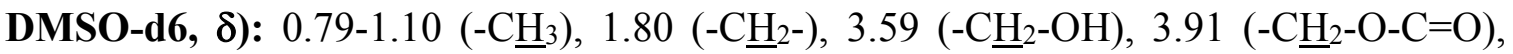

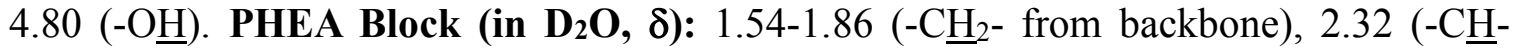
from backbone), $3.53(-\mathrm{O} \underline{\mathrm{H}}), 3.68\left(-\mathrm{C}_{2}-\mathrm{OH}\right), 4.08\left(-\mathrm{C}_{2}-\mathrm{O}-\mathrm{C}=\mathrm{O}\right)$.

The peak assignments for protons the ${ }^{1} \mathrm{H}-\mathrm{NMR}$ spectra of copolymers were made as shown below. PVP-b-12PMMA-b-PVP (in DMSO-d6, 8): 0.73-1.04 (- $\left.\underline{\mathrm{H}}_{3}\right), 1.40-1.82$

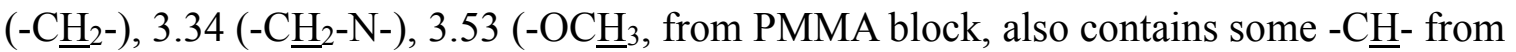

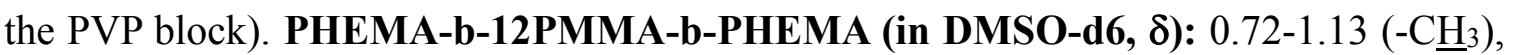
1.42-1.77 (- $\left.\underline{\mathrm{C}}_{2}-\right)$, 3.53 (-OC$\underline{H}_{3}$, from PMMA block, also contains $-\mathrm{C}_{2}-\mathrm{OH}$ from the PHEMA block), 3.87 (- $\underline{\mathrm{C}}_{2}-\mathrm{O}-\mathrm{C}=\mathrm{O}$ ). PHEA-b-12PMMA-b-PHEA (in DMSO-d6, $\delta$ ): 


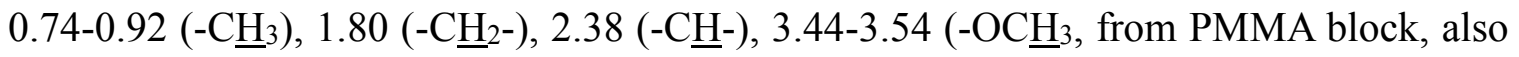
contains $-\underline{\mathrm{CH}}_{2}-\mathrm{OH}$ from the PHEA block), $3.98\left(-\underline{\mathrm{C}}_{2}-\mathrm{O}-\mathrm{C}=\mathrm{O}\right)$.

\subsubsection{Coupling of peptide to triblock copolymer}

The synthesized peptide was bonded to the triblock copolymer as shown in Scheme 2.4 using the following procedure. The copolymer $(0.25 \mathrm{~g})$ was dissolved in DIPEA/DMF (4.0 mL, 0.9 M DIPEA in DMF) at room temperature in a $25 \mathrm{~mL}$ three neck round bottom flask with magnetic stirring. The solution was degassed 15 min with nitrogen. HATU $(2.2 \mathrm{mg})$ was then added, to activate the terminal carboxylic acid groups of the copolymer, and stirred for $8 \mathrm{~min}$. P1 (0.022 g, $1.8 \times 10^{-2} \mathrm{mmol}, \sim 4$ molar excess over the terminal carboxylic acid groups) was dissolved separately in DMF $(2.0 \mathrm{~mL}$, in a glass vial), and then transferred via syringe to the reaction solution. The coupling reaction was carried out for 45 min under nitrogen atmosphere at room temperature. P2 was coupled separately to another batch of copolymer using the same procedure.

The peptide-functionalized copolymer was collected by precipitation into $40 \mathrm{~mL}$ of cold ethanol and centrifuged to remove un-reacted chemicals. The isolated solids were washed 3 times with $30 \mathrm{~mL}$ aliquots of cold ethanol and dried at $50{ }^{\circ} \mathrm{C}$ for $12 \mathrm{~h}$ under reduced pressure.

The peptide-functionalized copolymer conjugates are designated as follows: P1-PVP-b-12PMMA-b-PVP-P1, P1-PHEA-b-12PMMA-b-PHEA-P1, P2-PHEMA-b-12PMMA-b-PHEMA-P2 and respectively. The peptide-copolymer conjugates were characterized using FT-IR (Spectrum One, Perkin Elmer, Massachusetts, USA) to verify effective coupling reactions. 


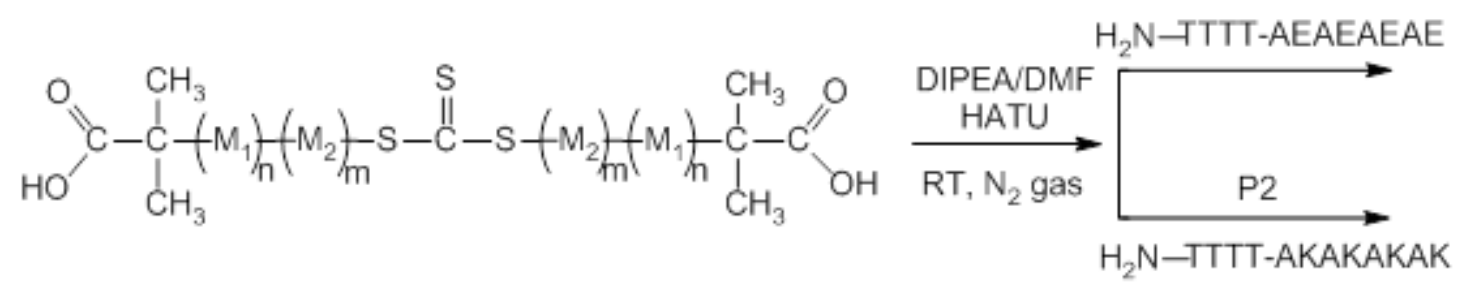

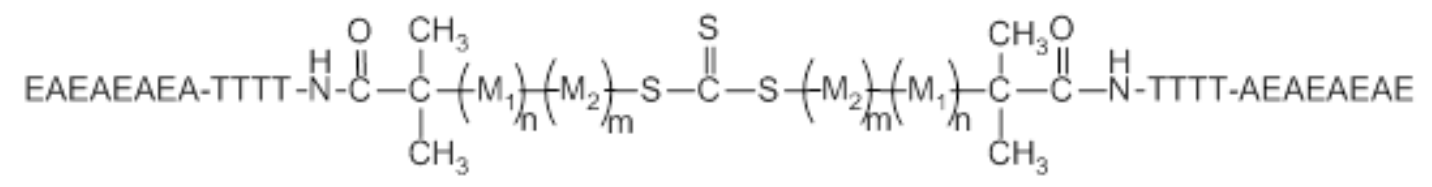

a. P1-copolymer conjugates

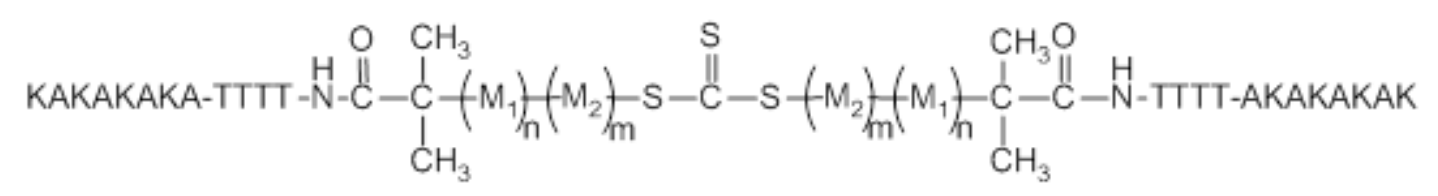

b. P2-copolymer conjugates

Scheme 2.4. Coupling reaction of $\mathrm{P} 1$ and $\mathrm{P} 2$ with amphiphilic copolymers.

\subsubsection{Self-assembly of peptide-copolymer conjugates in phosphate buffered saline solution (PBS) or deionized water (D.I. $\left.\mathrm{H}_{2} \mathrm{O}\right)$}

Peptide-copolymer conjugate self-assemblies were conducted in both sterile PBS

solution and D.I. $\mathrm{H}_{2} \mathrm{O}$. P1-PVP-b-12PMMA-b-PVP-P1 (11 $\left.\mathrm{mg}\right)$ and P2-PVP-b-12PMMA-b-PVP-P2 (11 mg) were dissolved together in DMSO (2 mL) by sonication and magnetic stirring to form a clear solution. The conjugate/DMSO solution was then slowly injected into PBS $(20 \mathrm{~mL}, \mathrm{pH}=7.4)$ via an insulin syringe (31 G syringe) with an injection speed of $\sim 0.4 \mathrm{~mL} / \mathrm{min}$ under magnetic stirring at $600 \mathrm{rpm}$. After the injection was completed, the suspension was stirred an additional $40 \mathrm{~min}$ to give a well-dispersed suspension. The nanoparticle suspension was allowed to settle for $10 \mathrm{~h}$, so that the self-assembled nanoparticles settled to the bottom layer giving a sponge-like appearance. The supernatant was replaced 4 times with fresh sterile PBS solution (or sterile D.I. $\mathrm{H}_{2} \mathrm{O}$ if the self-assembly was performed in D.I. $\mathrm{H}_{2} \mathrm{O}$ ) and then dialyzed $24 \mathrm{~h}$ in D.I. $\mathrm{H}_{2} \mathrm{O}$ to remove any DMSO residue. 
The self-assembled nanoparticle suspension was gently swirled and then $0.5 \mathrm{~mL}$ of the suspension was withdrawn using a $31 \mathrm{G}$ insulin syringe. The suspension was then slowly injected from the syringe while moving it across the surface of a freshly cleaned glass slide (washed by D.I. $\mathrm{H}_{2} \mathrm{O}$ and then immersed in $70 \% \mathrm{v} / \mathrm{v} E t O H / \mathrm{H}_{2} \mathrm{O}$ solution for $10 \mathrm{~h}$, and gently dried by kimwipe). As the syringe needle was moved slowly down the slide, a self-assembled nanoparticle fiber formed on the surface of the glass slide as the water in the PBS solution evaporated. This resulting nanoparticle fibers could be observed on the slide surface by optical microscopy (OLYMPUS BX60, Olympus America Inc., PA, USA), equipped with a camera (OPTIXCAM, Summit Series), power control (Olympus TH3) and observation software (TSview7). Self-assembly studies of peptide-functionalized PHEMA-b-12PMMA-b-PHEMA conjugates, and peptide-functionalized PHEA-b-12PMMA-b-PHEA conjugates were similarly carried out. The microscopy images of the self-assembled nanoparticle fibers and scaffolds are shown in Figure 2.4.

\subsubsection{Controlled release of Insulin from assembled nanoparticles}

Insulin was used as a model drug that was loaded into the different self-assembled nanoparticle compositions. Controlled release studies were performed for 22 days. Peptides- (P1- and P2-) functionalized PHEMA-b-12PMMA-b-PHEMA conjugates (1:1 $\mathrm{g} / \mathrm{g}$ ) were dissolved in DMSO $(3 \mathrm{~mL})$ to form a precursor solution $(14 \mathrm{mg}$ conjugate/mL DMSO), then insulin ( $0.063 \mathrm{mg}, 0.15 \mathrm{wt}$. \% based on conjugate matrix) was added to the precursor solution and mixed uniformly. The insulin-containing precursor solution was then slowly injected into sterile PBS to form self-assembled insulin-loaded conjugate nanoparticles, and allowed to settle for $10 \mathrm{~h}$.

Two types of controls were also tested. Peptide-functionalized PHEMA-b-12PMMA-b-PHEMA conjugate nanoparticles, without insulin, were prepared and used as controls to determine any 'background release' of peptides (P1 and P2) from 
the assembled conjugate nanoparticles. Also, a copolymer (PHEMA-b-12PMMA-b-PHEMA) without peptide-functionalization was loaded with the same quantity of insulin to give insulin-loaded copolymer nanoparticles to determine if the release rates of insulin from the peptide-conjugated nanoparticles differed from the non-peptide conjugated nanoparticles.

All self-assembled nanoparticle suspension was then dialyzed $24 \mathrm{~h}$ using dialysis tubing (Nominal MWCO 3,500, from Fisher Scientific) in PBS buffer solution, to remove free peptides and organic solvent. The assembled nanoparticle suspension was then collected and stored at $4{ }^{\circ} \mathrm{C}$.

The concentration of insulin-loaded conjugate nanoparticles, insulin-loaded copolymer nanoparticles, and conjugate nanoparticles without insulin, were all determined by gravimetric analysis, and found to be $1.8 \mathrm{mg} / \mathrm{mL}, 2.6 \mathrm{mg} / \mathrm{mL}$ and 2.0 $\mathrm{mg} / \mathrm{mL}$ respectively. For example, $3.0 \mathrm{~mL}$ of insulin-loaded conjugate nanoparticle suspension was vacuum dried to obtain $5.3 \mathrm{mg}$ solid mass, and thus the nanoparticle concentration was determined to be $1.8 \mathrm{mg} / \mathrm{mL}$. If using PBS solution, $3.0 \mathrm{~mL}$ of PBS solution was dried to get background mass, and thus net solid nanoparticle mass was obtained by taking off this background mass.

The desired quantity of insulin-loaded conjugate nanoparticles, insulin-loaded copolymer nanoparticles, and non-insulin-loaded conjugate nanoparticles, was measured based on the known concentration of these nanoparticles in PBS suspension, and transferred to a $15 \mathrm{~mL}$ centrifuge tube. After centrifugation to remove supernatant with free insulin, fresh D.I. $\mathrm{H}_{2} \mathrm{O}$ was added to set up controlled release tests carried out at 37 ${ }^{\circ} \mathrm{C}$ for 22 days. At the desired time intervals the supernatant with released insulin was separated from solid nanoparticles by centrifugation at $4000 \mathrm{rpm}$ for $15 \mathrm{~min}$. Then a specific amount of fresh sterile deionized water was added and the solids gently re-dispersed and left to stand until the subsequent release test. The concentration of the 
insulin in the collected supernatant was determined by micro-BCA protein assay according to the manual instructions.

The morphology of each of the different types of insulin-loaded conjugate nanoparticles was also characterized by optical microscopy to confirm nanoparticle fibers and 3D scaffolds, as shown in Figure 2.8.

\subsubsection{MTS cytotoxicity assayusing SW-620 cell lines}

The synthesized peptides, amphiphilic copolymers, and peptide-copolymer conjugates were tested for biocompatibility using SW-620 cell lines by standard MTS cytotoxicity assay. P1 was dissolved in DMSO/PBS (50 v/v \%) solution to form 2.8 $\mathrm{mg} / \mathrm{mL}$ of stock P1 solution, and P2 was dissolved in PBS solution to form $10.8 \mathrm{mg} / \mathrm{mL}$ of stock P2 solution. All three copolymer/DMSO precursor solutions were prepared and self-assembled in sterile D.I. $\mathrm{H}_{2} \mathrm{O}$, and then dialyzed for $24 \mathrm{~h}$ in D.I. $\mathrm{H}_{2} \mathrm{O}$ to form copolymer nanoparticle stock solutions with concentration ranging from $2.3 \mathrm{mg} / \mathrm{mL}$ to $2.7 \mathrm{mg} / \mathrm{mL}$. All three peptide-copolymer conjugate/DMSO precursor solutions were prepared and self-assembled into D.I. $\mathrm{H}_{2} \mathrm{O}$ and dialyzed $24 \mathrm{~h}$ in D.I. $\mathrm{H}_{2} \mathrm{O}$ to form conjugate nanoparticle stock solutions with concentration ranging from $2.5 \mathrm{mg} / \mathrm{mL}$ to 3.7 $\mathrm{mg} / \mathrm{mL}$.

SW-620 cells were seeded at a concentration of $\sim 1000$ cells per well in a 96-well culture plate and incubated for $16 \mathrm{~h}$ at $37{ }^{\circ} \mathrm{C}$ with $5 \% \mathrm{CO}_{2}$ in an humidified incubator. The media was removed and the cells were washed with fresh PBS the next day. Then fresh media with each sample solution was added to 96-well plate at a concentration of 1 , 10 and $100 \mu \mathrm{g} / \mathrm{mL}$ and each concentration was repeated with 6 replicates. Then the plate was incubated for $72 \mathrm{~h}$ at $37{ }^{\circ} \mathrm{C}$ with $5 \% \mathrm{CO}_{2}$ in the incubator. After $72 \mathrm{~h}$ of incubation, $20 \mu \mathrm{L}$ of MTS solution was added into each well and incubated again at $37{ }^{\circ} \mathrm{C}$ in the humidified incubator for $4 \mathrm{~h}$. Finally, the absorbance at $490 \mathrm{~nm}$ was recorded using an ELISA plate reader (BioTek Instruments Inc.). Three control solutions, PBS plus media, 
PBS/DMSO $50 \mathrm{v} / \mathrm{v} \%$ plus media, and pure media, were similarly subjected to the cytotoxicity test. For all samples, the final DMSO concentration was not more than $2 \%$ to avoid DMSO toxicity to cells. The samples are designated as follows: P1 and P2 are the two designed peptides; Copolymer NP1, NP2 and NP3 are nanoparticles assembled from copolymers of PVP-b-12PMMA-b-PVP, PHEMA-b-12PMMA-b-PHEMA, and PHEA-b-12PMMA-b-PHEA; Conjugate NP1, NP2 and NP3 are assembled conjugate nanoparticle fibers from peptide-copolymer conjugates of PVP-b-12PMMA-b-PVP, PHEMA-b-12PMMA-b-PHEMA, and PHEA-b-12PMMA-b-PHEA, respectively. 


\subsection{Results and Discussion}

\subsubsection{Design of peptides (P1 and P 2) for $\beta$-sheet self-assembly}

The rationale for the peptide design and coupling the peptides to amphiphilic triblock copolymers is to produce a peptide-directed self-assembly that undergoes multiple levels of assembly. Figure2.1a shows the complementary peptides labeled P1 and P2. When these are attached to amphiphilic copolymers, one batch conjugated to P1 and the other conjugated to P2 (labeled as P1-A-P1 and P2-B-P2 in Figure 2.1b), and the batches are combined, the chains undergo two levels of self-assembly. The amphiphilic block copolymer self-assembles into nanoparticles A and B, which need not be either the same size or same composition, while the complementary ionic peptides assemble these newly formed nanoparticles into continuous fibers of nanoparticles. These fibers can then further assemble to form a $\beta$-sheet in aqueous solution within the appropriate $\mathrm{pH}$ range by adding additional complementary peptide ( $\mathrm{P} 1$ and $\mathrm{P} 2$ with proper ratio. The broader implications of this are that multiple active agents can be introduced with appropriate compositions for desired release, both temporally and spatially.

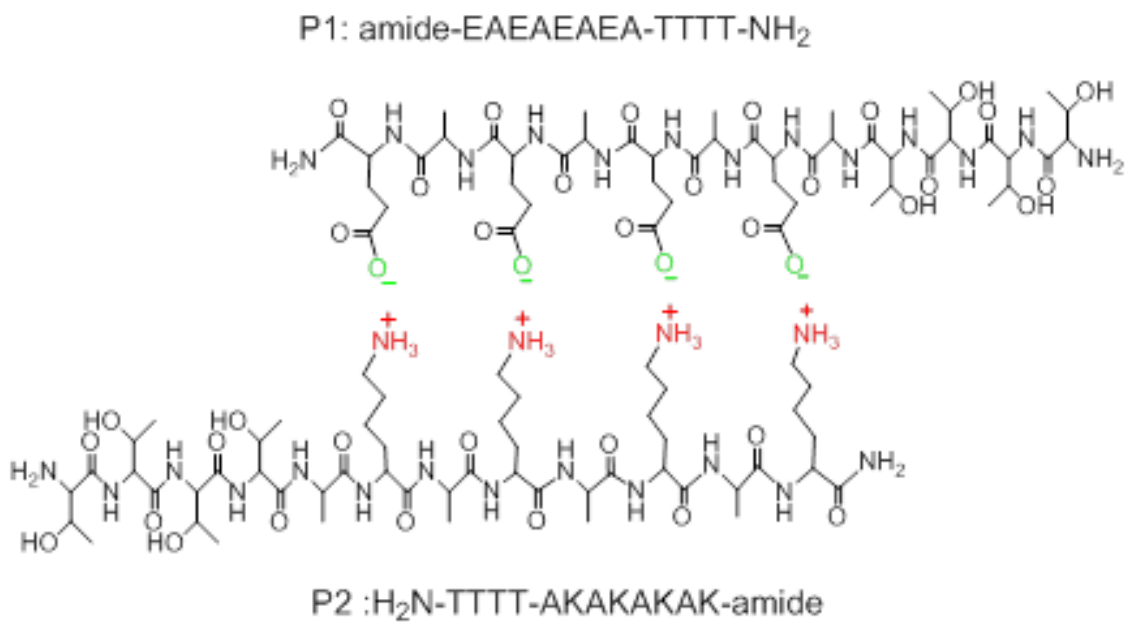

(a) 
Amphiphilic copolymer

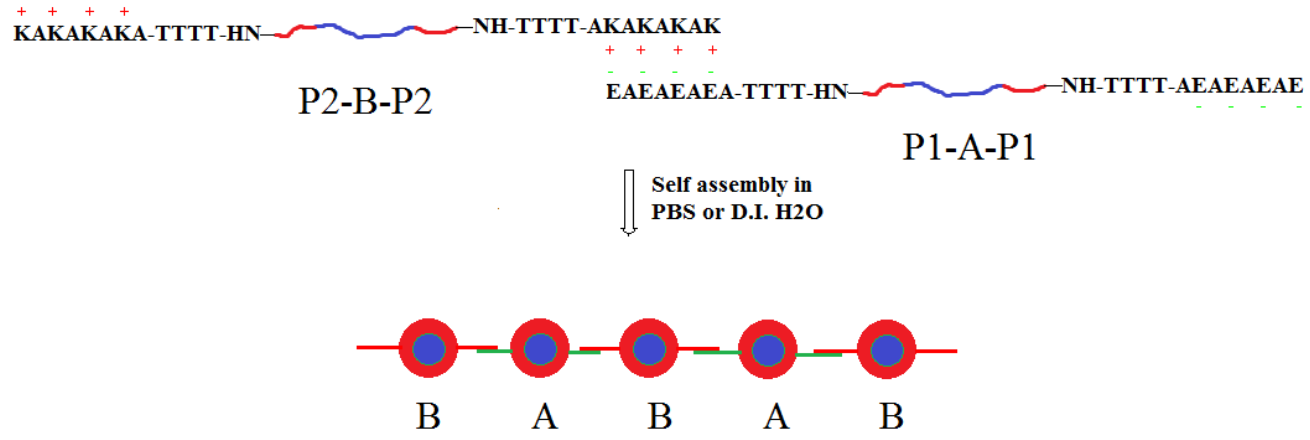

(b)

Figure 2.1. Illustration of (a) the complementary peptides, negatively charged P1 and positively charged $\mathrm{P} 2$, and (b) cartoon representation of two amphiphilic triblock copolymers, self assembling into core-shell nanoparticles A and B, and peptide linking of the nanoparticles into a polymeric "nanoparticle nanofiber" (1D) directed by self-assembly of P1 and P2. Higher assembly into 2D and 3D scaffolds is not shown.

Ionic complementary peptides showed excellent $\beta$-sheet assembly but were self-repulsive in aqueous solution (27). Using this principle, two peptide sequences, one positively charged and the other negatively charged (Figure 2.1a), were designed with alternating hydrophobic and ionic hydrophilic amino acids that could mutually self-assemble into $\beta$-sheet fibrils in aqueous solution.

The designed peptides were synthesized by semi-automated solid phase peptide synthesis strategy (section 2.2) and characterized by MALDI-TOF MS (Figure 2.1S a,b) to confirm the molecular weight of $1246.42\left[\mathrm{M}+\mathrm{Na}^{+}\right]$for $\mathrm{P} 1$ and $1220.01\left[\mathrm{M}+\mathrm{H}^{+}\right]$for P2, showing excellent agreement with the theoretic molecular weight of P1 (MW: 1223.25) and P2 (MW: 1219.45). The chemical structure of P1 and P2 were further confirmed by ${ }^{1} \mathrm{H}-\mathrm{NMR}$ analysis (Figure 2.2 a,b) to show desired chemical structure. 
P1: amide-EAEAEAEA-TTTT- $\mathrm{NH}_{2}$
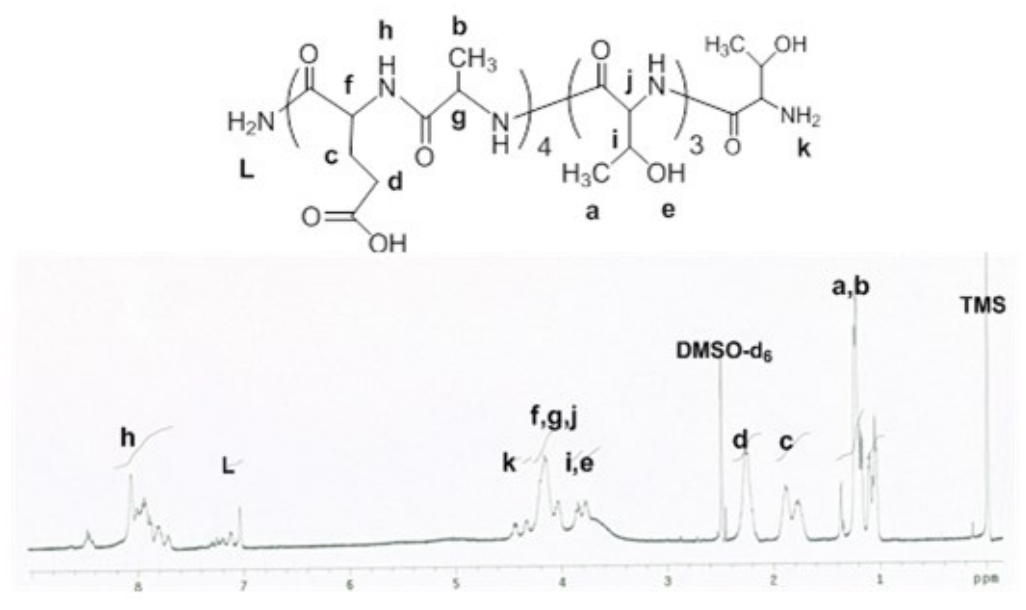

(a)

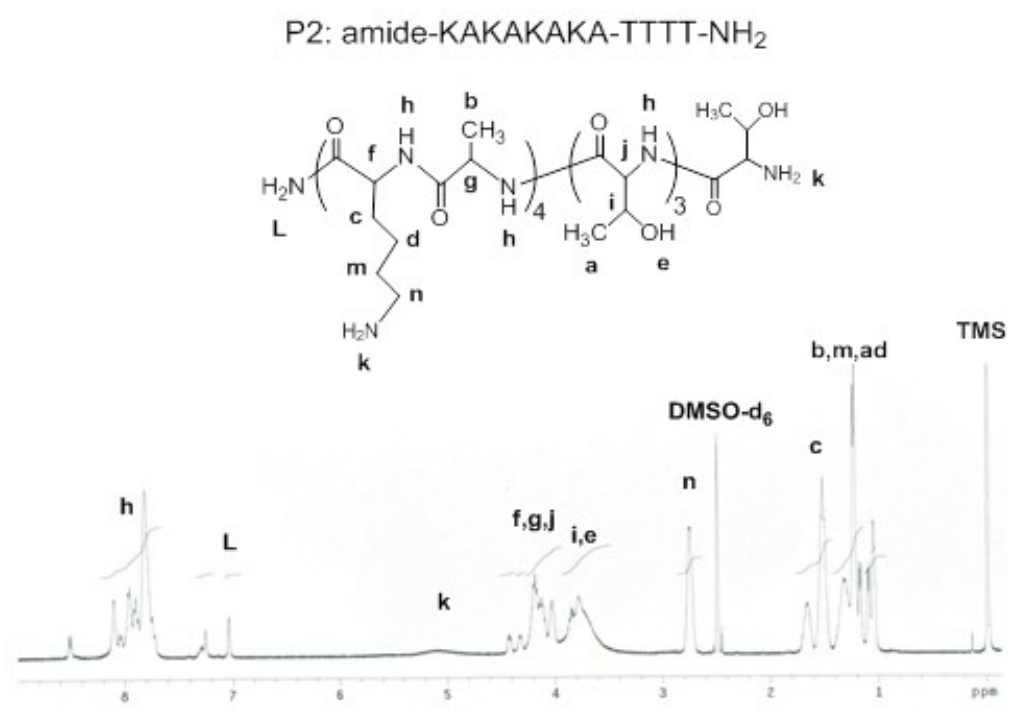

(b)

Figure 2.2. ${ }^{1} \mathrm{H}-\mathrm{NMR}(\mathrm{DMSO}-\mathrm{D} 6+1 \% \mathrm{TMS})$ spectra of the synthesized P1 (a) and P2 (b).

Each peptide sequence was designed to include two parts (Scheme 2.1), the self-assembly part bearing complementary electrostatic charges, and the spacer part, using hydrophilic amino acid (Threonine), that is designed to separate the self-assembly 
part from the amphiphilic block copolymer by increasing its solubility, and its mobility to facilitate coupling with the complementary peptide units. P1 is designed for negative charges from glutamic acid (E), which bears carboxylic acids that ionize when the $\mathrm{pH}$ is above 4.4. P2 has lysine (K), which has an amine side group that carries positive charges when the $\mathrm{pH}$ is below 10.0 (27). Therefore, glutamic acid and lysine will electrostatically interact with each other when the $\mathrm{pH}$ is between $4.4 \sim 10.0$ in aqueous (i.e. PBS) solution. This interaction contributes the main driving force for self-assembly, as illustrated in Figure2.1a. The hydrophobic alanine (A) also contributes to the self-assembly between P1 and P2 by burying its own hydrophobic domain of alanine side change.

The use of electrostatic interaction to drive the self-assembly is preferred because they are stronger than either hydrogen-bonding interactions or hydrophobic interactions, and so will dominate the assembly process (28). This is illustrated by the different self-assembly behavior of the designed peptides in DMF/PBS solution, shown in Figure 2.3. Figure 2.3a and $\mathbf{b}$ show a solution of $P 1$ and $P 2$ while Figure $3 c$ shows a combined solution of $\mathrm{P} 1$ and $\mathrm{P} 2$. The solution of $\mathrm{P} 1$ alone exhibits $\mathrm{H}$-bonding and can form viscous gelation when concentration is at $1.1 \mathrm{wt} . \%$, while P2 exhibits only a solution with significant flow at same concentration. The combination of P1 and P2 forms a viscous, non-flowing gel at a lower concentration $(0.55$ wt. $\%+0.55$ wt. $\%)$, showing stronger interaction than either P1 or P2 alone. 


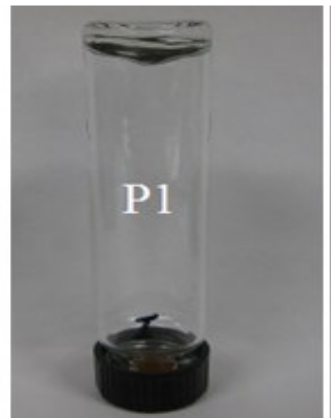

(a)

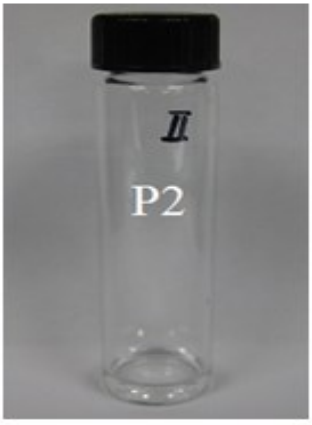

(b)

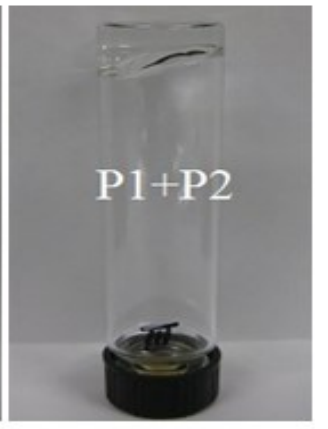

(c)

Figure 2.3. Photographs of the gels formed by $\mathrm{P} 1, \mathrm{P} 2$ and a mixture of $\mathrm{P} 1$ and $\mathrm{P} 2$ in DMF/PBS co-solvent (1:1 v/v) where (a) P1 (1.1 wt.\%) gels in $1 \mathrm{~mL}$ of DMF/PBS; (b) P2 (1.1 wt.\%) flows in $1 \mathrm{~mL}$ of DMF/PBS, able to flow; and (c) a strong gel is formed after mixing a $1 \mathrm{~mL}$ solution of P1 with a $1 \mathrm{~mL}$ of $\mathrm{P} 2$ solution (giving $0.55 \mathrm{wt} . \%$ of each peptide in $2 \mathrm{~mL}$ DMF/PBS).

\subsubsection{Synthesis and characterization of amphiphilic tri-block copolymers}

Reversible addition-fragmentation chain transfer (RAFT) polymerization was used to synthesize the amphiphilic tri-block copolymer with reactive carboxylic acid groups at both chain ends. This was done using BDAT as the chain-transfer agent (CTA) and AIBN as the initiator (Scheme 2.3) (29). BDAT was selected because it is an efficient CTA for many monomers. This gives us the ability to produce a range of ABA triblock copolymers to assemble core-shell nanoparticles that are suitable for many different active agents. Furthermore, BDAT is a highly efficient CTA giving reactive carboxylic acid terminals, which is required so that peptide conjugation is efficient, thereby giving efficient assembly into fibers.

Before the copolymer synthesis the structure and purity of BDAT was confirmed by ${ }^{1} \mathrm{H}-\mathrm{NMR}\left(\mathrm{in} \mathrm{CDCl}_{3}\right)$. The proton peaks of the $-\mathrm{CH}_{3}(\mathrm{~s}, 12 \mathrm{H})$ at $1.67 \mathrm{ppm}$ and $-\mathrm{COOH}(\mathrm{s}$, $2 \mathrm{H})$ at $13.0 \mathrm{ppm}$, are in agreement with the reported chemical structure (26). 
The first step of the polymerization for all the copolymers in this work was the synthesis of the hydrophilic block, so that the shell of the final copolymer nanoparticle was hydrophilic. Thus, after polymerizing the appropriate monomer, using BDAT and AIBN, to give the PVP, PHEMA, or PHEA in high yield (Table 2.1), the polymer was used as a macro-CTA to synthesize the PMMA hydrophobic inner block in the second step, giving the desired tri-block copolymer.

${ }^{1} \mathrm{H}$-NMR analysis confirmed the existence of all the appropriate peaks for the hydrophilic block and the block copolymer (Figure 2.4). For example, the ${ }^{1} \mathrm{H}-\mathrm{NMR}$ spectrum of PVP (in $\mathrm{D}_{2} \mathrm{O}$ ) (Figure 2.4a), shows peaks for protons d ( $\delta$ : 1.46-1.61), $\mathrm{f}(\delta$ : 2.18-2.32), $\mathrm{g}(\delta: 1.90), \mathrm{h}(\delta: 3.19)$ and e $(\delta: 3.52-3.66)$ with the ratio of the integrated areas of d:f:g:h:e $=23.11: 22.29: 23.16: 21.59: 9.85 \cong 2: 2: 2: 2: 1$. The number average molecular weight $\left(\mathrm{M}_{\mathrm{N}}\right)$ analyzed by MALDI-TOF MS (Table 2.1) was found to be similar for PVP and PHEA, but slightly lower for PHEMA. Accordingly the $\mathrm{X}_{\mathrm{N}}$, calculated from the $\mathrm{M}_{\mathrm{N}}$, was found to be 78,74 , and 66 for PVP, PHEA, and PHEMA respectively. Considering the symmetrical structure of BDAT, these repeat units are evenly distributed at both sides of CTA and therefore the structure of the macro-CTA is represented as: $\quad$ (PVP) ${ }_{39}$-BDAT-(PVP) 39, $_{37} \quad$ (PHEA) ${ }_{37-B D A T-(P H E A)} 37$ and (PHEMA) 33-BDAT-(PHEMA) 33 respectively.

The amphiphilic block copolymer was synthesized by combining the macro-CTA with MMA and fresh AIBN. The ratio of the blocks in the final tri-block copolymer was determined from ${ }^{1} \mathrm{H}$ NMR (Figure 2.4). An example calculation is given for PVP-b-PMMA-b-PVP (Figure 4b) in section 2.4 with equation to calculate the actual block ratio of $\mathrm{X}_{\mathrm{N}}(\mathrm{PVP}): \mathrm{X}_{\mathrm{M}}(\mathrm{PMMA})$ to be 1:4.9. The block ratio $\left(\mathrm{X}_{\mathrm{N}}: \mathrm{X}_{\mathrm{M}}\right)$ of PHEMA-b-PMMA-b-PHEMA and PHEA-b-PMMA-b-PHEA were calculated in a similar manner from the ${ }^{1} \mathrm{H}-\mathrm{NMR}$ spectra (Figure $2 \mathrm{~S}$ a,b and Figure $3 \mathrm{~S}$ a,b, with corresponding equation) and found to be 1:11 and 1:8.7 respectively. From these data the 
$\mathrm{X}_{\mathrm{N}}$ of the hydrophilic blocks and $\mathrm{X}_{\mathrm{M}}$ of the hydrophobic PMMA block for all three

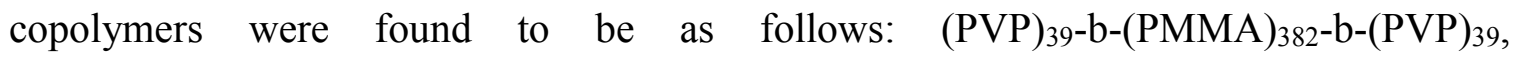

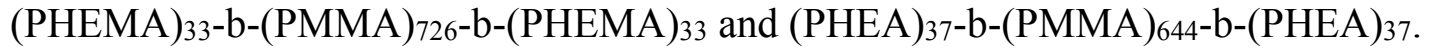

The high ratio of PMMA used relative to the hydrophilic monomer was chosen to ensure that sufficiently large core-shell nanoparticles would be obtained to allow the nanoparticles to be visualized by optical microscopy. In fact, the water-swollen particles that were produced when dripped into water were found to be spherical and possess a diameter near $1 \mu \mathrm{m}$, as shown in Figure 2.6. Smaller ratios of PMMA produce accordingly smaller particles that have a diameter between 100 to $500 \mathrm{~nm}$ by dynamic lighter scattering test.

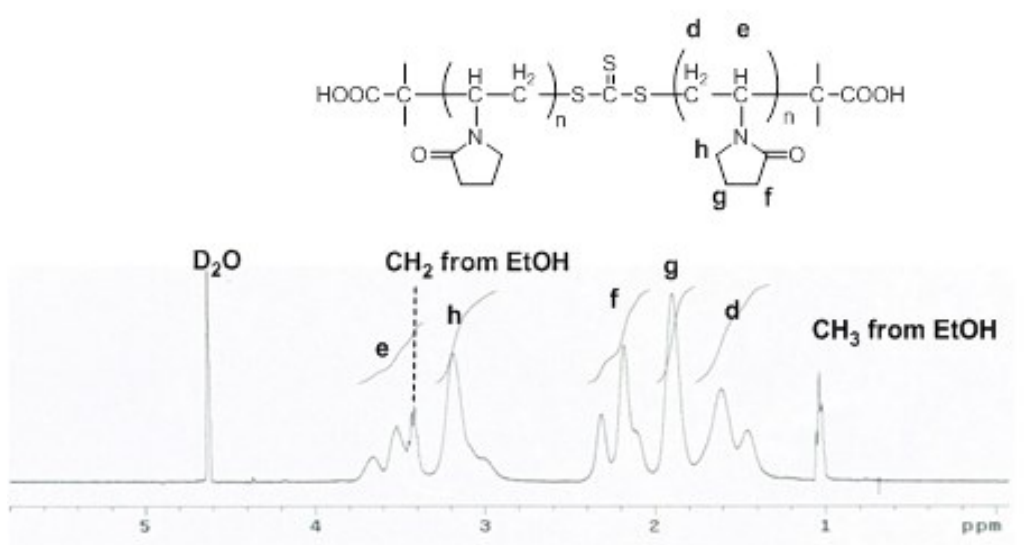

(a) 


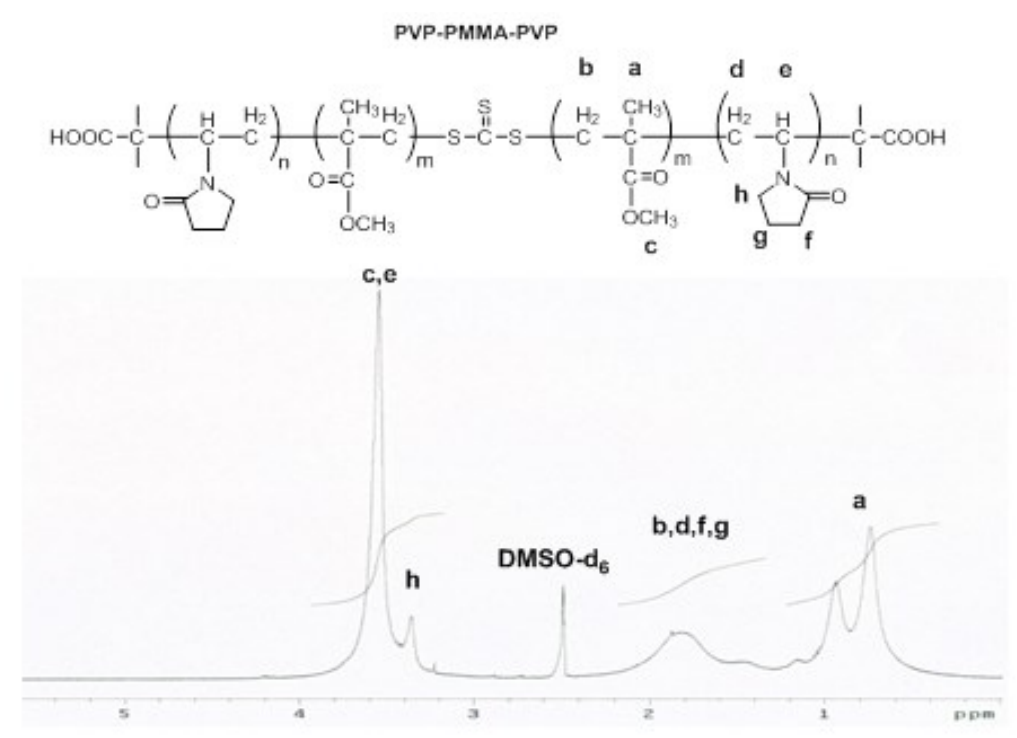

(b)

Figure 2.4. ${ }^{1} \mathrm{H}-\mathrm{NMR}$ spectra (in DMSO-d6) of (a) the synthesized hydrophilic polymer PVP and (b) tri-block copolymer PVP-b-12PMMA-b-PVP by RAFT polymerization.

\subsubsection{Coupling peptides to copolymers}

The carboxylic acid groups at the amphiphilic copolymer chain ends were coupled to the amine group of the P1 and P2 peptides is illustrated in Scheme 2.4. The peptide-functionalized copolymer conjugates were purified and vacuum-dried, and the coupling was confirmed by FT-IR (Figure 2.5).

Figure 2.5 shows the $\mathrm{P} 1$ and $\mathrm{P} 2$ absorption bands of the carbonyl stretching vibrations $(\mathrm{C}=\mathrm{O})$ at $\sim 1670 \mathrm{~cm}^{-1}$ and the amide nitrogen-hydrogen $(\mathrm{O}=\mathrm{C}-\mathrm{N}-\mathrm{H})$ bending vibrations at $\sim 1530 \mathrm{~cm}^{-1}$. The FT-IR spectra of the peptide-coupled copolymer conjugates also show the absorption peaks from peptides between $1526 \mathrm{~cm}^{-1}$ to $1678 \mathrm{~cm}^{-1}$, which differs from the polymer carbonyl absorption at $1720 \mathrm{~cm}^{-1}$. These same peptide bands were also seen in the other peptide-coupled conjugate polymers shown in the Supplemental Information (Figure 2.4S a,b).These data confirm the peptide coupling to the copolymers. 


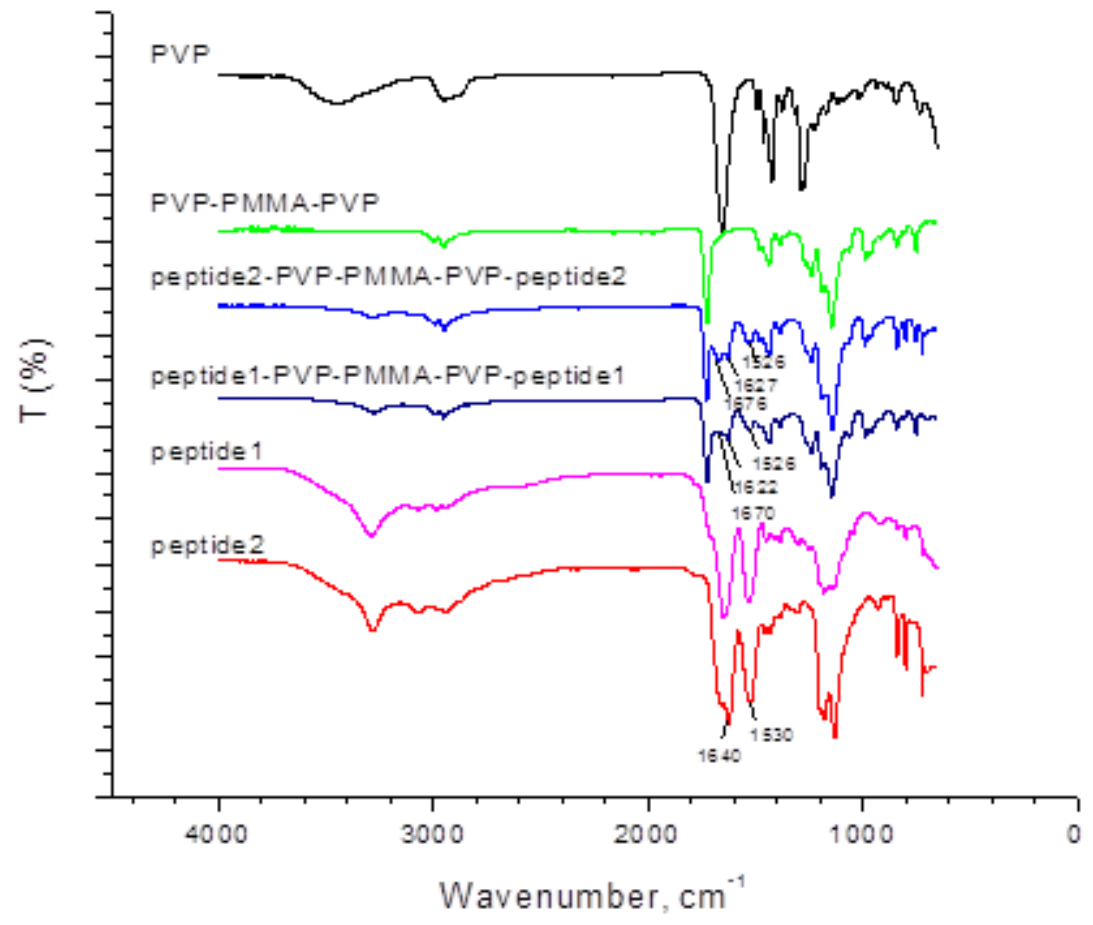

Figure 2.5. Comparison of FT-IR spectra of the synthesized copolymer, P1(Peptide1), P2(Peptide2), and Peptide-PVP-b-12PMMA-b-PVP conjugates.

\subsubsection{Self-assembly of peptide-copolymer conjugates in aqueous solution}

The purpose of the copolymer design is to prepare self-assembled controlled-release "nanoparticle fibers" that can subsequently self-assemble to produce 3D scaffolds as directed by $\beta$-sheet peptide assembly (Figure 2.1b). By this method, the mixing of two batches of complementary peptide-conjugated polymeric nanoparticles (A and $\mathrm{B})$ results in their self-assembly into an ordered arrangement of nanoparticle fibers and 3D scaffolds. This simple 2-part system has the advantage of allowing each nanoparticle to be customized for a desired active ingredient(s) and also allowing the nanoparticle fibers to simultaneously serve as a two-drug delivery system and flexible scaffolds for cell migration and proliferation. Using controlled ratios and sequential addition multi-drug 
delivery systems are achievable in the desired sequence within the resulting particle fibers.

Self-assembled nanoparticle fibers and 3D scaffolds, are shown for a two-drug delivery system in representative microscopic images in Figure 2.6 and 2.7, in aqueous media (PBS solution or D.I. $\mathrm{H}_{2} \mathrm{O}$ ). Figure 2.6a-c shows PVP-b-12PMMA-b-PVP, after the complementary peptide-conjugated nanoparticles were combined. The initially formed fibers are observed with $\mathrm{t} \sim 0-10 \mathrm{~h}$, with the extent of the assembly increasing further with $\mathrm{t}>10 \mathrm{~h}$ after combining the complementary peptide-copolymer conjugate pair, and finally a 3D assembly is observed. Figure 2.7 a,b shows PHEMA-b-12PMMA-b-PHEMA in deionized water with $\mathrm{t} \sim 10 \mathrm{~h}$, and (b) peptide-coupled PHEA-b-12PMMA-b-PHEA in PBS at $t \sim 10 \mathrm{~h}$. The self-assembly process appeared to progress somewhat more rapidly in PBS solution than in D.I. $\mathrm{H}_{2} \mathrm{O}$. This is thought to be due to the presence of the alkaline ions in PBS solution being beneficial to the self-complementary assembly of the designed peptides (3). Although PBS is often better than D.I. $\mathrm{H}_{2} \mathrm{O}$ for peptide dissolution, for these assembled nanoparticle fibers PBS buffer media facilitates the formation of long NP fibers on glass slide surfaces when compared to results using D.I. $\mathrm{H}_{2} \mathrm{O}$ media. There may be other differences between the two media, but in this work none were noted.

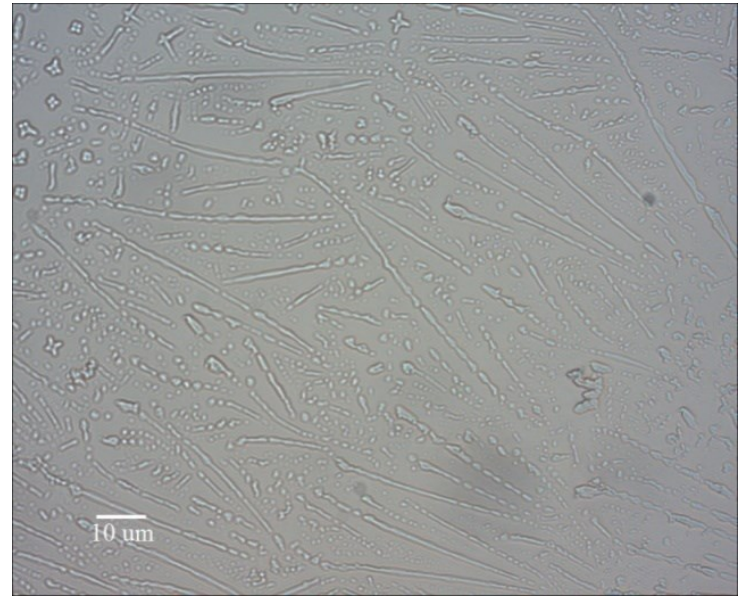

(a)

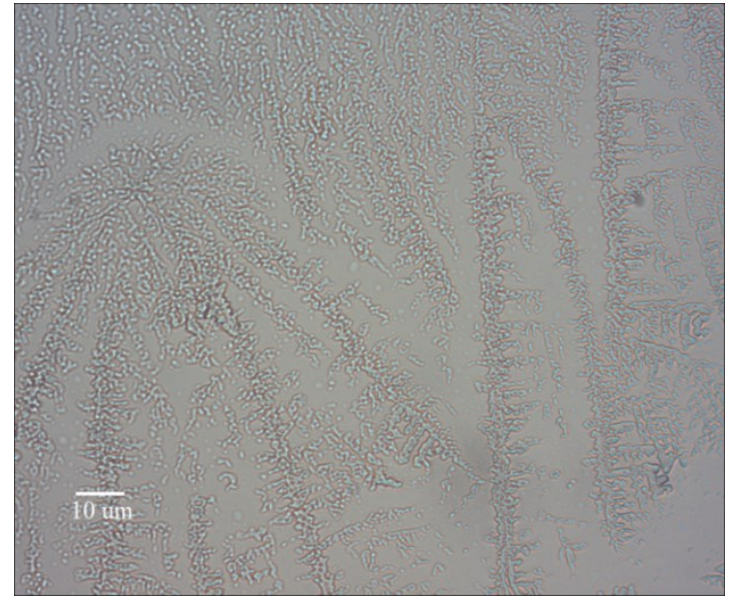

(b) 


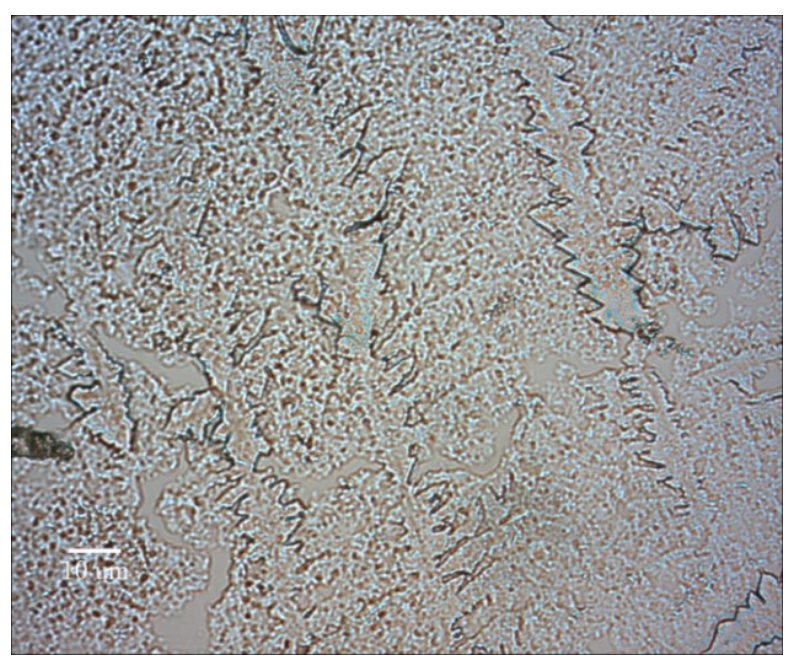

(c)

Figure 2.6. Conjugate NP fibers from peptide-coupled PVP-b-12PMMA-b-PVP in PBS with self-assembly times of (a) $\sim 10 \mathrm{~h}$ showing fibers, (b) $\mathrm{t}>10 \mathrm{~h}$, showing further assembly and (c) t $>10 \mathrm{~h}$, showing 3-D scaffolds.

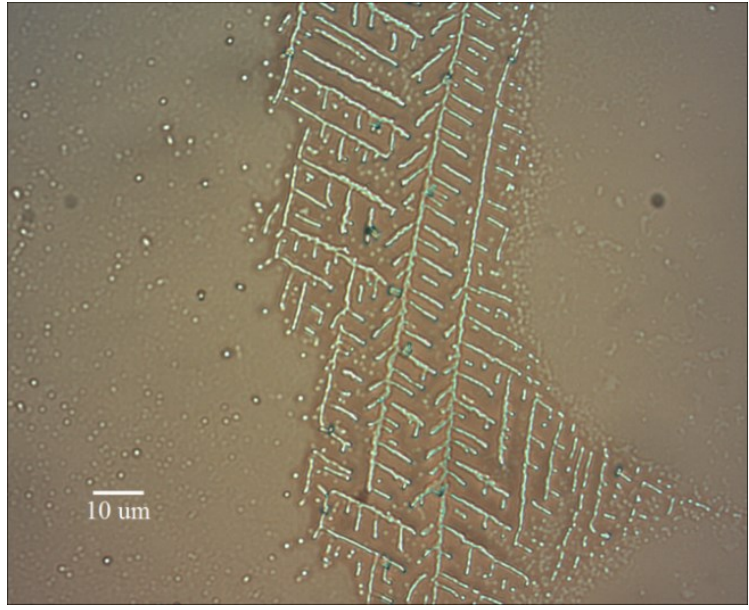

(a)

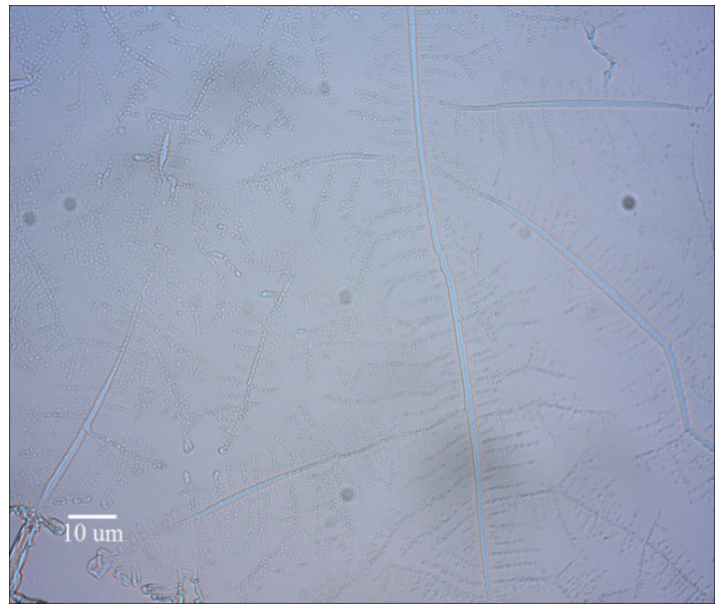

(b)

Figure 2.7. Conjugate NP fibers from (a) peptide-coupled PHEMA-b-12PMMA-b-PHEMA in deionized water with $\mathrm{t} \sim 10 \mathrm{~h}$, and (b) peptide-coupled PHEA-b-12PMMA-b-PHEA in PBS with $\mathrm{t} \sim 10 \mathrm{~h}$.

This approach has substantial versatility and is effectively a fundamental technology with significant control over each level of assembly. For example, any approach can be used to design the nano/microparticle composition and structure, as either a 
homopolymer or a copolymer, with the advantage of an amphiphilic copolymer being that it will self-assemble into a core-shell design. This allows the particle interior to be designed to be suitable for a desired cargo such as an active ingredient, and to release the active ingredient at a desired rate, while the particle exterior can be designed to be stable in the delivery medium. The core and shell can then be selected for an appropriate release rate for the specific active ingredient. The peptide assembly also offers significant level of control, not only in the design of the assembly motif and its stability, but also in how the assembly manipulates the nano/microparticle arrangement. The assembly can be designed to couple so that all the nano/microparticles can carry the same drug, but release the drug at very different rates over an extended release time, or different particles can be assembled so that multiple drugs can be released from different nano/micro particles with each particle being released at a rate appropriate for that drug. This can be done for small molecules or in principle for proteins.

Another block copolymer design was made using poly(ethylene glycol) methyl ether acrylate as the hydrophilic monomer to give a hydrophilic shell that readily forms a hydrogel. Our copolymer design, PPEG-b-12PMMA-b-PPEG, like the others, can serve as controlled release devices but in this case the outer shell, being ethylene glycol based, would resist protein adsorption and cell adhesion. The same peptide bonding motifs that are already described, $\mathrm{P} 1$ and $\mathrm{P} 2$, were used. The peptide-copolymer conjugates were placed in a DMF precursor solution $(13 \mathrm{mg} / \mathrm{mL})$ to self-assemble into a dense 3D nanoparticle scaffold by adding $2 \mathrm{~mL}$ of deionized water. The freeze-dried scaffold was characterized by FESEM and images are shown in Figure 2.8a. The same composition was tested, but with P1 and P2 simply being blended into the mixture rather than bonded to the amphiphilic PPEG-12-PMMA-PPEG copolymer (Figure 2.8b).The results clearly show the effect of bonding the peptides to the nanoparticles on the assembly process. The 
overall density of the scaffolding can be manipulated by changing composition and peptide length.

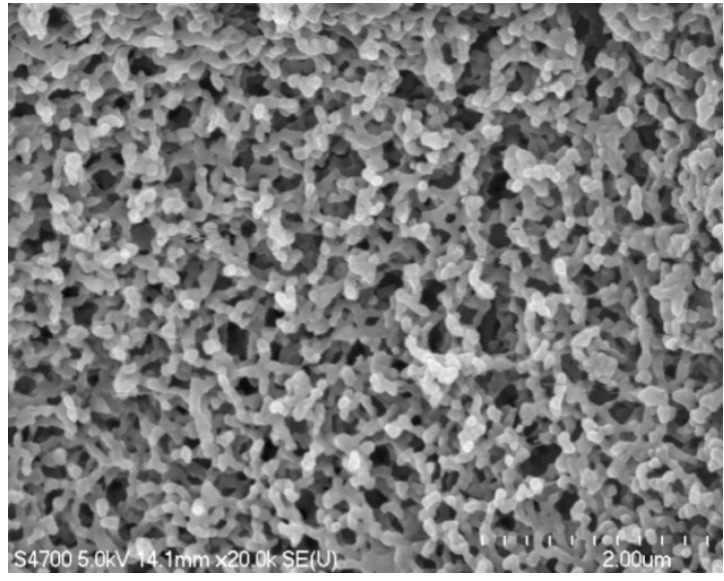

(a)

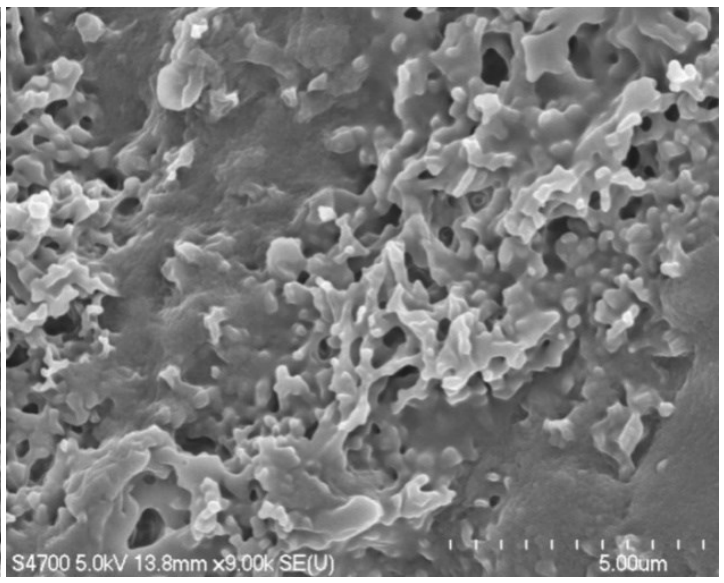

(b)

Figure 2.8. FESEM images show comparison of self-assembled scaffold morphologies (a) self-assembled from peptide-coupled PPEG-b-12PMMA-b-PPEG conjugates and (b) nanoparticle scaffolds control self-assembled from peptide physically mixed with PPEG-b-12PMMA-b-PPEG.

\subsubsection{Controlled release study of Insulin as model drug}

The advantage and rationale for the use of an amphiphilic block copolymer is to allow nanoparticles (or microparticles) to be prepared with a suitable composition to control the release rate for a desired drug, with the shell allowing the nano/microparticles to be stable in a given medium, and to serve as a tissue scaffold, or resist protein adsorption. In a preliminary test of the ability of assembled nanoparticles to serve as controlled release devices, insulin was used to test the ability to release a model protein.

Insulin was loaded into the synthesized peptide-copolymer conjugates at a theoretical content of 0.15 wt.\%. After combining aqueous solutions of complementary peptide-functionalized copolymer nanoparticles, each containing insulin, long 
self-assembled nanoparticle fibers and 3D scaffolds were formed, as the representative images show in Figure 2.9.

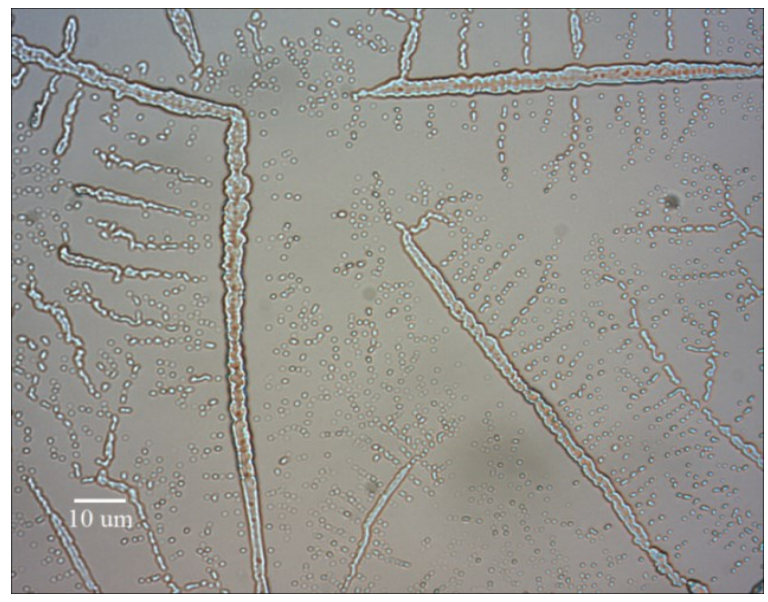

(a)

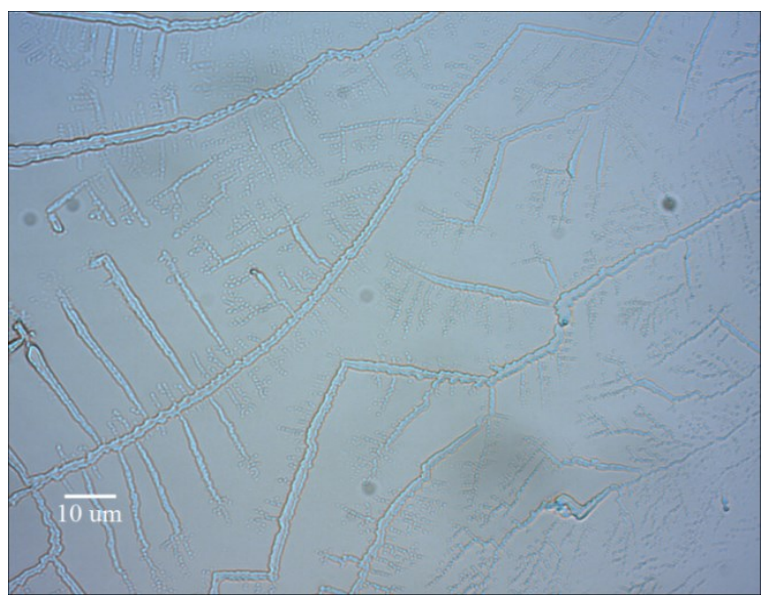

(c)

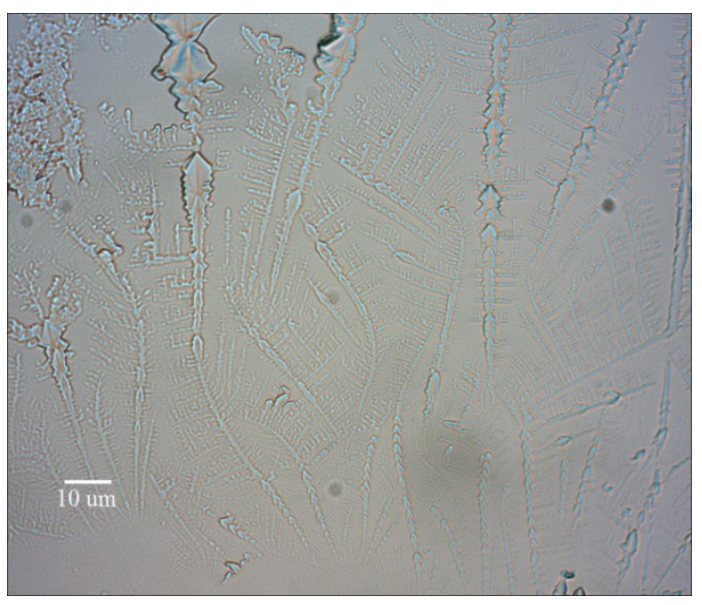

(b)

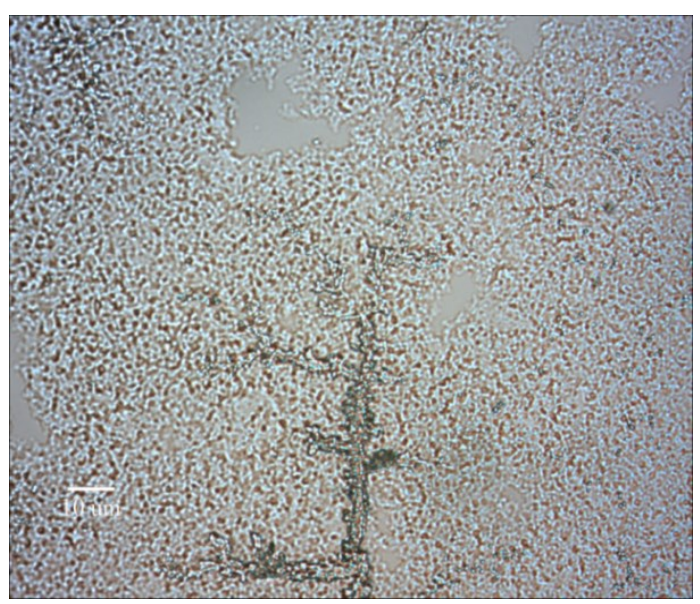

(d)

Figure 2.9. Optical microscopy images of insulin-loaded conjugate nanoparticle fibers and 3D scaffolds, self-assembled in PBS solution. Insulin-loaded nanoparticle fibers of (a) peptide-coupled PVP-b-12PMMA-b-PVP conjugates self-assembled in PBS with assembly time $\mathrm{t} \sim 10 \mathrm{~h} ; \quad$ (b) peptide-coupled PHEMA-b-12PMMA-b-PHEMA conjugates in PBS with $\mathrm{t} \sim 10 \mathrm{~h}$; (c) peptide-coupled PHEA-b-12PMMA-b-PHEA conjugates with $\mathrm{t} \sim 10 \mathrm{~h}$; and (d) representative insulin-loaded nanoparticle scaffolds at condensed phase with $\mathrm{t}>10 \mathrm{~h}$. Images of (a), (b), (c) were prepared on a glass slide surface by gently shaking self-assembled conjugate nanoparticles to form a uniform suspension and then 
transferring to the glass slide surface. Image (d) is of self-assembled nanoparticle scaffolds from peptide-coupled PVP-b-12PMMA-b-PVP conjugates with self-assembly time $>10 \mathrm{~h}$ without shaking the suspension, used as the representative morphology of $3 \mathrm{D}$ nanoparticle scaffolds.

The self-assembled insulin-loaded nanoparticle fibers from peptide coupled PHEMA-12PMMA-PHEMA were used in a controlled release test run for 22 days at 37 ${ }^{\circ} \mathrm{C}$. The released insulin solution was collected by centrifugation and the quantity was determined by micro-BCA test. The cumulative quantity of insulin released over 22 days is shown in Figure 2.10. The release is compared with insulin-loaded PHEMA-12PMMA-PHEMA nanoparticles alone, and for the peptides from peptide coupled PHEMA-12PMMA-PHEMA without insulin.

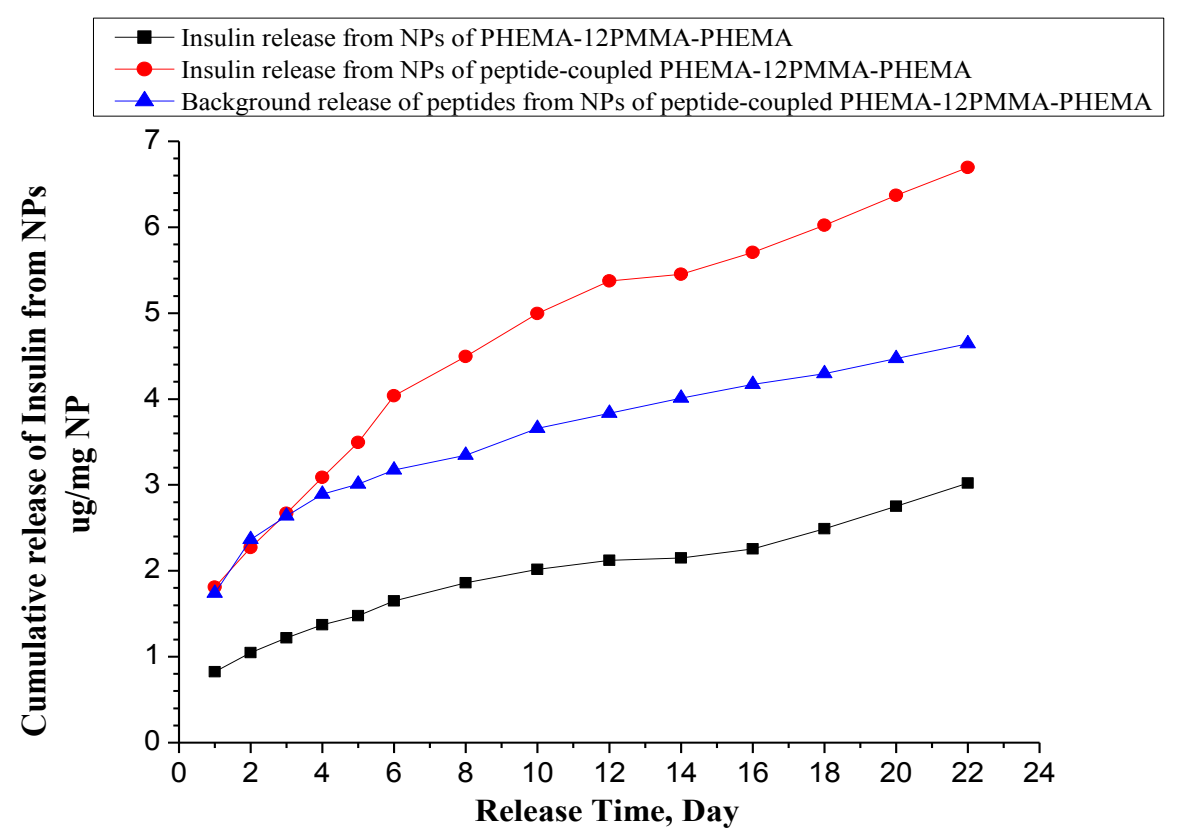

Figure 2.10. Comparison of insulin released from PHEMA-12PMMA-PHEMA copolymer nanoparticle control and assembled peptide-coupled 
PHEMA-b-12PMMA-b-PHEMA conjugate nanoparticle fibers tested by micro-BCA protein assay.

The results show a steady release of insulin from the assembled conjugate nanoparticle fibers over a three-week period. A 'background' release is measured (blue triangles) that is due to residual peptides (P1 and $\mathrm{P} 2)$ remaining in the formulation after the synthesis of the peptide-copolymer conjugates. Unfortunately, the assay does not differentiate the insulin from the residual peptides, but even accounting for this release, the data show that the conjugated peptide nanoparticle fibers give a controlled insulin release over a three-week period thatis slightly faster than the release from the copolymer nanoparticle controls.

\subsubsection{Biocompatibility study on SW-620 cell lines by MTS assay}

The cytotoxicity of the synthesized peptides, copolymers and peptide-copolymer conjugates were measured by MTS assay using SW-620 cell lines. The results are shown in Figure 2.11. The cells incubated with media alone or with PBS and/or DMSO were used as control and the results showed absorption intensity ranging from 0.64 to 0.88 at $490 \mathrm{~nm}$. When absorptions were compared to that of the controls, MTS test for all synthesized peptides, copolymers and peptide-copolymer conjugates showed similar or slightly higher absorption intensity at $490 \mathrm{~nm}$. These results suggest that SW-620 cells cultured in the presence of synthesized biomaterial solution or nanoparticle suspension with concentration range at $1 \sim 100 \mu \mathrm{g} / \mathrm{mL}$ are not adversely affected by these synthesized biomaterials. 
MTS assay of the synthesized peptide-copolymer conjugates, copolymers and peptides at cell concentration of $\sim 1000$ per well.

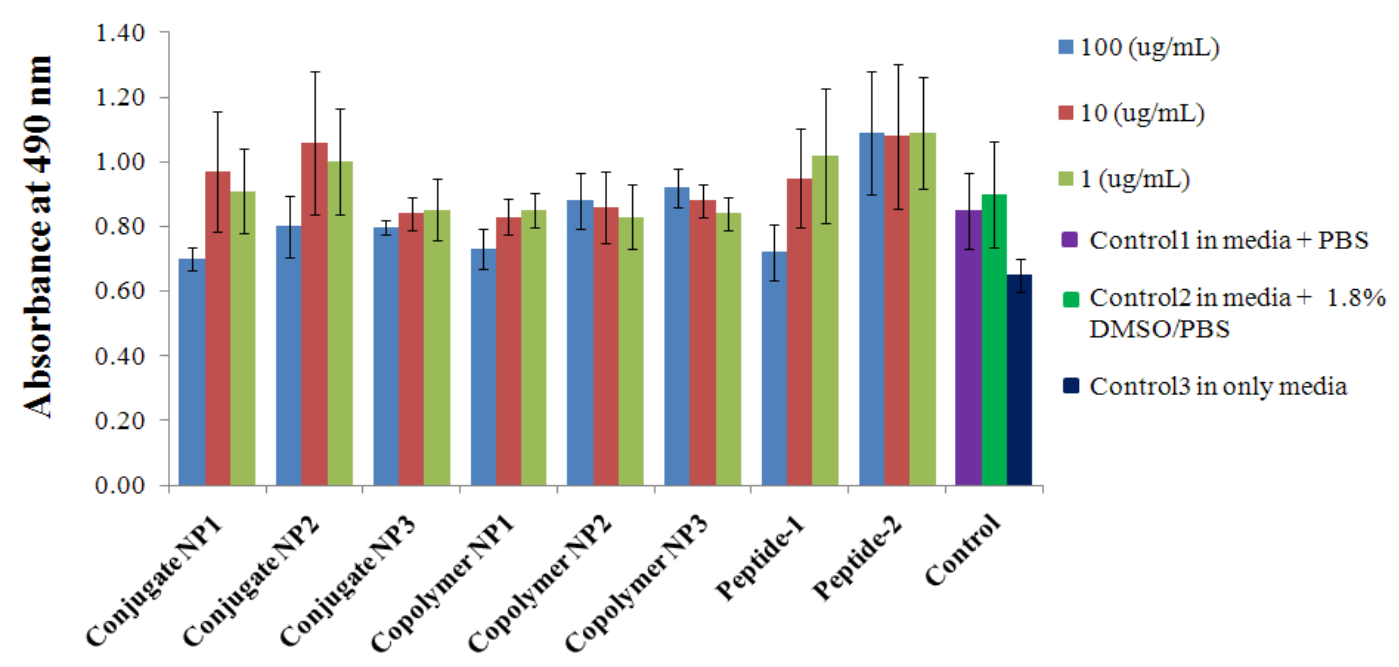

Figure 2.11. Cytotoxicity test on SW-620 cell lines by MTS assay.

Conjugates NP1, NP2 and NP3 represent self-assembled nanoparticles from peptide-coupled copolymers (PVP-b-12PMMA-b-PVP, PHEMA-b-12PMMA-b-PHEMA and PHEA-b-12PMMA-b-PHEA). Copolymer NP1, NP2 and NP3 are nanoparticles for the non peptide-coupled copolymers. Peptide- 1 and Peptide-2 are the synthesized peptides (P1 and P2).

\subsection{Conclusion}

A novel fundamental technology was proved in this work that showed how peptide-directed self-assembly can be used to direct the assembly of individual polymeric nanoparticles into fibers and 3D scaffolds. This technology combines the advantages of versatile controlled release systems and flexible scaffolds for tissue engineering. In this work a single drug, insulin, was tested, but the broader implications of this technology are that multiple drugs can be loaded into the scaffolding and the release rate of each drug can be separately controlled by the selection of the nanoparticles used to form the scaffold. This is because different polymer compositions and structures (e.g. core-shell 
morphology) can be employed, and the assembly of fundamentally different nano- and microparticles can be controlled. Although only three different copolymers were described in this work, the technology allows other polymers to be used, and as long as the peptides can be coupled to the terminals to induce the assembly process, polymers can be prepared using any step growth or chain growth process. 


\section{Main Findings in This Chapter:}

In this chapter, we proved the hypothesis that ionic complementary peptide-functionalized amphiphilic copolymer could self-assemble into polymeric nanoparticle fibers and 3D scaffolds with or without model drug loading. This was done by functionalizing several sets of amphiphilic triblock copolymers with two oppositely charged peptides (P1 and P2), including PVP-b-PMMA-b-PVP, PHEMA-b-PMMA-b-PHEMA and PHEA-b-PMMA-b-PHEA, with reactive carboxylic acid terminals synthesized by RAFT polymerization using BDAT as CTA. Controlled release study using insulin as model drug showed sustained release of insulin over 3 weeks from self-assembled nanoparticle scaffolds. Cytotoxicity test on SW-620 cell lines showed all synthesize peptides, copolymers and peptide-copolymer conjugates were biocompatible with SW-620 cell lines at concentration below $100 \mu \mathrm{g} / \mathrm{mL}$.

\section{Acknowledgements:}

The authors thank the Department of Chemistry for financial support of this project, and Mr. Jerry Anzalone of the Department of Material Science and Engineering for training in the use of optical microscopy analysis. 


\section{References}

[1] Loo Y, Zhang S, Hauser CAE. From short peptides to nanofibers to macromolecular assemblies in biomedicine.Biotechnol. Adv. 2012;30: 593-603.

[2] Zhang S, Holmes T, Lockshin C, Rich A. Spontaneous assembly of a self-complementary oligopeptide to form a stable macroscopic membrane. Proc. Natl. Acad. Sci. U. S. A. 1993; 90: 3334-38.

[3] Ramachandran S, Tseng Y, Yu B Y. Repeated rapid shear-responsiveness of peptide hydrogels with tunable shear modulus. Biomacromolecules 2005;6: 1316-21.

[4] Nagai Y, Unsworth LD, Koutsopoulos S, Zhang S. Slow release of molecules in self assembling peptide nanofiber scaffold. J. Controlled Release 2006;115:18-25.

[5] Koutsopoulos S, Unsworth LD, Nagai Y, Zhang S. Controlled release of functional proteins through designer self-assembling peptide nanofiber hydrogel scaffold. Proc. Natl. Acad. Sci. U. S. A. 2009;106:4623-8.

[6] Horii A, Wang X, Gelain F, Zhang S. Biological designer self-assembling peptide nanofiber scaffolds significantly enhance osteoblast proliferation, differentiation and 3-D migration. PLoS One 2007;2:e190.

[7] Gelain F, Unsworth LD, Zhang S. Slow and sustained release of active cytokines from self-assembling peptide scaffolds. J. Controlled Release 2010;145:231-9.

[8] Galler KM, Aulisa L, Regan KR, D'Souza RN, Hartgerink JD. Self-assembling multidomain peptide hydrogels: designed susceptibility to enzymatic cleavage allows enhanced cell migration and spreading. J. Am. Chem. Soc. 2010;132:3217-23.

[9]Petkar KC, Chavhan SS,Agatonovik-Kustrin S, Sawant KK. Nanostructured materials in drug and gene delivery: A review of the state of the art. Crit. Rev. Ther. Drug Carrier Syst. 2011; 28(2): 101-64.

[10] Hunter C, Elsom J, Wibroe P, Moghimi M. Polymeric particulate technologies for oral drug delivery and targeting: a pathophysiological perspective. 
Nanomed-Nanotechnol 2012; 8:S5-S20.

[11]Miller T, Hill A, Uezguen S, Weigandt M, GoepferichA. Analysis of immediate stress mechanisms upon injection of polymeric micelles and related colloidal drug Carriers: implications on drug targeting. Biomacromolecules 2012; 13: 1707-18.

[12]Ding H, Wang X, Zhang S, Liu X. Applications of polymeric micelles with tumor targeted in chemotherapy. J. Nanopart.Res.2012; 14:1254 (P1-13).

[13]Khanbabaie R, Jahanshahi M. Revolutionary impact of nanodrug delivery on neuroscience. Curr.Neuropharmacol. 2012; 10(4): 370-92.

[14]Vauthier C, Bouchemal K. Methods for the preparation and manufacture of polymeric nanoparticles. Pharm. Res. 2009; 26(5): 1025-58.

[15]Boyer C, Stenzel MH, Davis TP. Building nanostructures using RAFT polymerization.J. Polym. Sci. Pol. Chem. 2011; 49(3):551-95.

[16]Butruk B, Trzaskowski M, Ciach T. Fabrication of biocompatible hydrogel coatings for implantable medical devices using Fenton-type reaction. Mater. Sci. Eng., C 2012; 32:1601-09.

[17]Guiseppi-Elie A, Dong C, Dinu CZ. Crosslink density of a biomimetic poly(HEMA)-based hydrogel influences growth and proliferation of attachment dependent RMS 13 cells. J. Mater. Chem. 2012;22: 19529-39.

[18]Lin M, Xu P, Zhong W, Preparation, characterization, and release behavior of aspirin-loaded poly(2-hydroxyethyl acrylate)/silica hydrogels. J. Biomed. Mater. Res. B- 2012;100B(4):1114-20.

[19]Li Z, Kawamura K, Kawashita M, Kudo T, Kanetaka H, Hiraoka M. In vitro assessment of poly(methylmethacrylate)-based bone cement containing magnetite nanoparticles for hyperthermia treatment of bone tumor, J. Biomed.Mater.Res. A 2012;100A(10):2537-45.

[20]Nicolas J, Mantovani G, Haddleton DM. Living radical polymerization as a tool for 
the synthesis of polymer-protein/peptide bioconjugates. Macromol.Rapid Commun. 2007;28(10): 1083-111.

[21]Ryadnov MG, Cherny DI. Natively unfolded state for engineering nanoscale fibrillar arrays.Macromol.Biosci.2012; 12: 195-201.

[22]Sharma N, Top A, Kiick KL, Pochan DJ. One-dimensional gold nanoparticle arrays by electrostatically directed organization using polypeptide self-assembly.Angew.Chem.Int. Ed. 2009; 48: 7078-82.

[23]Tanaka M, Abiko S, Himeiwa T, Nakamura M, Koshikawa N, Kinoshita T. Two-dimensional self-assembly of amphiphilic peptide at the solid/water interface toward a facile method for metal nanoparticle alignment. Chem. Lett. 2012; 41: $1221-22$.

[24]Nisbet DR, Williams RJ. Self-assembled peptides: Characterisation and in vivo response. Biointerphases 2012; 7(1-4): 2.

[25]Webber MJ, Matson JB, Tamboli VK, Stupp SI. Controlled release of dexamethasone from peptidenanofiber gels to modulate inflammatory response. Biomaterials 2012; 33(28): 6823-32.

[26]Lai JT, Filla D, Shea R. Functional polymers from novel carboxyl-terminated trithiocarbonates as highly efficient RAFT agents. Macromolecules 2002; 35: 6754-56.

[27]Ulijn RV, Smith AM. Designing peptide based nanomaterials. Chem. Soc. Rev. 2008; 37: $664-75$.

[28]Zou D, Tie Z, Lu C, Qin M, Lu X, Wang M, Wang W, Chen P. Effects of hydrophobicity and anions on self-assembly of the peptide EMK16-II. Biopolymers 2009; 93(4): 318-29.

[29]Boyer C, Stenzel MH, Davis TP. Building nanostructures using RAFT polymerization.J. Polym. Sci. Pol. Chem. 2011; 49(3): 551-95. 
Smart Self-Assembly of Peptide-Functionalized Amphiphilic Copolymer Nanoparticles: Novel Fibers and 3D Scaffolds with Ability for Multiple Drug Loading and Independent Controlled Release

XiaochuDing ${ }^{\mathrm{a}}$, Trevor Moser ${ }^{\mathrm{b}}$, Qi Gao ${ }^{\mathrm{b}}$, TolouShokuhfar ${ }^{\mathrm{b}}$, Patricia A. Heiden ${ }^{\mathrm{a} *}$, aDepartment of Chemistry, Michigan Technological University, Houghton, MI 49931, USA

The idea for nanoparticle fibers was conceived by Dr. Heiden. The peptide designs, the experimental designs, and research were conducted by Mr. Xiaochu Ding, except for the cytotoxicity testsusing NIH3T3 cell linesthatwere conducted by Mr. Trevor Moser in Dr. Tolou Shokuhfar's lab, and the FESEM micrographs that were taken by Mr. Qi Gao.

Mr. Xiaochu Ding

Department of Chemistry

Michigan Technological University

Mr. Qi Gao

Department of Mechanical Engineering and Engineering Mechanics

Michigan Technological University
Mr. Trevor Moser

Department of Mechanical Engineering and Engineering Mechanics Michigan Technological University

Dr. Tolou Shokuhfar

Department of Mechanical Engineering and Engineering Mechanics Michigan Technological University

Dr. Patricia A. Heiden*

Department of Chemistry

Michigan Technological University 


\section{Chapter 3}

Smart Self-Assembly of Peptide-Functionalized Amphiphilic Copolymer Nanoparticles: Novel Fibers and 3D Scaffolds with Ability for Multiple Drug Loading and Independent Controlled Release XiaochuDing ${ }^{\mathrm{a}}$, Trevor Moser ${ }^{\mathrm{b}}$, Qi Gao ${ }^{\mathrm{b}}$, TolouShokuhfar ${ }^{\mathrm{b}}$, Patricia A. Heiden ${ }^{\mathrm{a} *}$, aDepartment of Chemistry, Michigan Technological University, Houghton, MI 49931, USA

${ }^{b}$ Department of Mechanical Engineering and Engineering Mechanics, Michigan Technological University, Houghton, MI 49931, USA

*Corresponding author: +1 906487 3452; fax: +1 9064872061.

Email address:paheiden@mtu.edu (P.A. Heiden).

This manuscript is in submission to Macromolecular Bioscience. 


\section{Abstract}

Conventional self-assembled peptide nanofiber scaffolds lack the ability to be simultaneously loaded with multiple drugs that have different chemical and physical properties, to allow individual controlover the release of each of the loaded drugs, and to provide uniform, or controlled but non-uniform (e.g. gradient), distribution of the drugs within the same scaffold system. In this work, a novel ionic complementary peptide-functionalized polymeric nanoparticle was designed and self-assembled into 1D, 2D, and 3D polymeric nanoparticle scaffolds. Such self-assembled nanoparticle scaffolds have the potential to overcome each of the above-described limitations of conventional scaffolds. This new method allows the assembly of individual nanoparticles into a continuous fiber scaffold. Because of this design, each nanoparticle composition can be selected to allow it to be loaded with the desired drug and release that drug at an appropriate rate. In principle this approach also gives control over the spatial distribution of the drug-containing nanoparticles to allowblocks of nanoparticles with a given drug, or nanoparticle gradients to be formed in thescaffolds, while also yielding an injectable system. This process gives unprecedented flexibility into the design and preparation of the scaffold. The self-assembled nanoparticle scaffold formation was confirmed by optical microscopy and FESEM micrographs. Several model drugs, including hydrophobic and hydrophilic small molecules, were loaded in the scaffold system showing controllable and sustainable release over 3 weeks. The self-assembled 2D scaffold membrane was incubated with fibroblast cells (NIH3T3 cell lines) in a culture dish that demonstrated non-toxicity and non-inhibition to the cell proliferation. This type of nanoparticle scaffold combines the advantages of the precision associated with peptide self-assembly and the versatility of polymeric nanoparticle controlled release systems for a new type of tissue engineering.

Keywords: peptide, self-assembly, nanoparticle scaffold, multi-drug controlled release 


\subsection{Introduction}

An efficient tissue growth is usually dependent on the delivery of various drugs (i.e. active biomedicine, growth factors) to cells within tissue regeneration. Thus there are important scientific relationships between tissue engineering and scaffolding system with drug delivery (1). For example, skin regeneration usually requires a complex delivery of growth factors and cytokines, such as fibroblast growth factor, keratinocyte growth factor, vascular endothelial growth factor and interleukin $1 \alpha$, to release within the wound bed to promote cell proliferation and migration to achieve wound healing $(\mathbf{2 , 3})$. Therefore, there is a need to fabricate a more sophisticated system with multi-drug delivery abilities within scaffolding for tissue engineering.

Conventional polymeric hydrogels (both synthetic and natural polymers) have been widely developed to incorporate a single drug in scaffold system for tissue regeneration, for example, physically or chemically incorporating bioactive ingredients in

poly(ethylene glycol) (PEG) hydrogels (4,5), poly(lactide)-b-poly(ethylene oxide)-b-poly(lactide) (PLA-b-PEO-b-PLA) or poly(lactic-co-glycol acid) (PLGA) hydrogels $(6,7)$, or natural biomaterial gels (e.g. alginate, collagen, chitosan, gelatin) (8-14). Most recently, Caicco et al. (15) developed a physically blended hydrogel composite system of hyaluronan-methylcellulose (HAMC) as for the localized delivery and sustainable release of CyclosporinA (CsA), a promising neuroprotective and neuroregenerative agent for neural stem/progenitor cells, for treatment of stroke. The authors incorporated $\mathrm{CsA}$ in HAMC gel by three different methods and compared the release rate. The three incorporation types include a solubilized type (CsA/acetonitrile solution mixed with HAMC gel), a particulate type (CsA solid particulate dispersed in MC solution and then embedded in HAMC gel), and polyl(actic-co-glycolic acid)(PLGA) microsphere-encapsulated CsA type (CsA encapsulated in PLGA microspheres and then embedded in HAMC gel). Interestingly, the in-vitro controlled release test showed that solubilized CsA type released from HAMC gel only for 2 days, particulate type expanded 
to 7-10 days, but CsA from PLGA microsphere-encapsulated type showed sustainable release over 21 to 28 days. Most importantly, the CsA released from PLGA microspheres retained bioactivity that was equivalent to that of fresh CsA when compared by a neurosphere assay (15). However, similarly with most of the other polymeric hydrogel scaffolds, this study is also limited to single drug incorporation.

Self-assembled peptide nanofiber scaffolds have been gaining in popularity compared to traditional scaffolding for tissue engineering because of non-toxicity, biodegradability, and the porosity of fibril structures is similar to extracellular matrix (ECM) for cell attachment. Also, these materials assemble in situ into a hydrogel at physiological environment (16). The first generation self-assembling designer peptide, EAK16-II (AEAEAKAKAEAEAKAK), was discovered in a yeast protein, zuotin (17). This type of ionic complementary peptide spontaneously self-assembles into stable $\beta$-sheets in aqueous conditions across a broad range of temperature and $\mathrm{pH}$, and even in the presence of a high concentration of the denaturing agents urea and guanidium hydrochloride $(\mathbf{1 8 , 1 9 )}$. The self-assembling forces are the hydrophobic interactions of alanine (A) domains and ion-pair interactions between negatively charged glutamic acid (E) side chains with positively charged lysine $(\mathrm{K})$ side chains. That this strong ion-pair interaction contributes to stable $\beta$-sheet formation was further supported by investigation of a complementary pair of designer peptides, the self-repulsive but mutually attractive peptide sequences that possessed positive charges (Ac-WKVKVKVKVK-amide) and negative charges (Ac-EWEVEVEVEV-amide) (20). On mixing this pair of complementary peptides, a rapid assembly into a viscoelastic hydrogel occurred at a concentration as low as $0.25 \mathrm{wt} . \%$. This hydrogel retained mechanical strength, even after repeated shear-induced breakdowns, due to the electrostatic interactions. The strong electrostatic and selective interaction between the opposite charges demonstrated one of the key merits of using ion-complementary $\beta$-sheet motifs. 
These designer self-assembling peptide hydrogels (e.g. EAK16-II, RADA16-I or II) have been widely used as both controlled drug delivery systems and 3D scaffolds. The drug delivery capability is typically done by physically incorporating a drug into the peptide scaffold during the gel formation $(\mathbf{2 1 , 2 2})$, but it can also be chemically bonded onto the C-terminal or N-terminal (23), or sometimes combination of both methods to

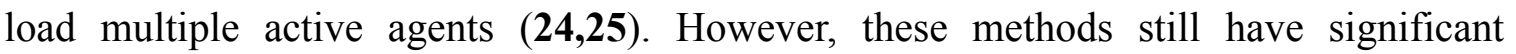
limitations with respect to the quantity of drug(s) that can be incorporated, but furthermore the incorporation of $\operatorname{drug}(\mathrm{s})$ may have an impact on the subsequent self-assembly process or the mechanical stability of the formed hydrogel, and there is very limited ability to control the release of drugs with different properties. Therefore, the hydrogel itself must be designed in conjunction with the specific drug(s) that will be incorporated. Moreover, the effective distribution of multiple drugs in scaffolds is not easy to achieve.

To address these limitations, we designed and reported a new type of ionic complementary peptide-directed self-assembly of polymeric nanoparticle fibers and 3D scaffolds to be a more powerful and versatile technique to allow multiple drug incorporation within a scaffolding system and to allow the release rate of each drug to be controlled appropriately (26). This new type of designed scaffold having multiple drug loading ability was achieved by functionalizing an amphiphilictriblock copolymer with two oppositely charged peptides (P1-ABA-P1 and P2-ABA-P2, ABA: triblock copolymer, $\quad \mathrm{P} 1$ : $\quad \mathrm{H}_{2} \mathrm{~N}-\mathrm{TTTT}-\mathrm{AEAEAEAE}-\mathrm{CONH}_{2} \quad$ and $\mathrm{P}$ : $\mathrm{H}_{2} \mathrm{~N}-\mathrm{TTTT}-\mathrm{AKAKAKAK}-\mathrm{CONH}_{2}$ ), respectively. While the peptides controlled the nanoparticle assembly the copolymer composition gave domains that self-assembled into polymeric micro or nanoparticles for use as drug carriers that also possess the peptide functionalities (P1-NP-P1 and P2-NP-P2). These nanoparticles then further self-assemble into 'nanoparticle fibers' and eventually lead to 3D scaffolds with a 'sponge-like' 
appearance in aqueous solution.

In the present work, we continue the study of the self-assembly behavior and test the versatility of the methodology with a series of designed peptide-functionalized copolymer (P1-ABA-P1 and P2-ABA-P2) with different particle size, and with additional peptides (P1 and P2) to adjust the scaffold porosity and mechanical stability. Multiple drug loading and control of the drug releasing rate from different nanoparticle compositions are demonstrated in this work using several small molecules as model drugs, including hydrophobic molecule (4',5'-dibromofluorecein), moderately hydrophobic molecule (nitrofurazone) and slightly water soluble hydrophilic molecule (amoxicillin). The biocompatibility test is conducted by incubating the self-assembled 2D scaffold membrane with fibroblast cells (NIH3T3 cell lines) in a humidified incubator at $37{ }^{\circ} \mathrm{C}$ for 2 weeks, showing non-toxicity and non-inhibition to the cell proliferation and migration. The self-assembly of peptide-functionalized nanoparticles with additional host peptides (P1 and P2) and with different concentration is also conducted to form nanoparticle composite peptide hydrogels, to compare mechanical stability and morphology. 


\subsection{Experimental Section}

\subsubsection{Materials}

All reagents for peptide synthesis were purchased from AAPPTec LLC (Louisville, KY) and used as received. Cleavage reagents, including trifluoroacetic acid (TFA, 99\%), thioanisole (99\%) and anisole (99\%), were purchased from Sigma-Aldrich, and 1,2-ethanedithiol (98.0\%) was from Fluka. 1-Vinyl-2-pyrrolidinone (VP) ( $\geq 99 \%)$, 2-hydroxyethyl methacrylate (HEMA) (97\%), methyl methacrylate (MMA) (99\%), 1,4-dioxane (99+\%), 2,2'-azobisisobutyronitrile (AIBN) (98\%), dimethyl formamide (DMF, 99.9\%) and phosphate buffered saline (Biotech) were purchased from Sigma-Aldrich. Ethyl alcohol (200 proof, anhydrous) was from PHARMCO-AAPER, and diethyl ether (anhydrous) and dimethyl sulfoxide (DMSO) were from Mallinckrodt Baker Inc. (Phillipsburg, USA). Model drugs of 4',5'-Dibromofluorescein (DBF) and cell adhesion peptide (RGDS) were from Sigma-Aldrich, nitrofurazone powder and amoxicillin capsules were from Jungle Laboratories Corporation and Shopko pharmacy (Made by Sandoz International, Germany, NDC code: 007881-2613-01). DBF and nitrofurazone were used as received, amoxicillin powder was removed from the capsules and was purified by dissolving in ethanol and removing undissolved components, and recrystallized before use. VP and HEMA were purified prior to use by passing through a neutral alumina column. MMA was distilled before use. All other reagents were used as received. Fibroblast cells (NIH3T3 cell line) were purchased from ATCC and cell media (DMEM/High Glucose) was from Hyclone Laboratories, Inc. (Utah, USA). Ultrapure deionized water $(>17.6 \mathrm{M} \Omega-\mathrm{cm})$ was obtained from MEG-PURE SYSTEM (MP-190 LC).

\subsubsection{Synthesis and self-assembly of ionic complementary peptides (P1 and P2)}

Two ionic complementary peptides, $\mathrm{P} 1\left(\mathrm{H}_{2} \mathrm{~N}-\mathrm{TTTT}\right.$-AEAEAEAE-amide $)$ and $\mathrm{P} 2$ $\left(\mathrm{H}_{2} \mathrm{~N}\right.$-TTTT-AKAKAKAK-amide), were synthesized by a standard semi-automatic solid 
phase peptide synthesis strategy (Endeavor 90I, AAPPTec LLC, Louisville, KY, USA), using Fmoc chemistry, on a rink amide resin. A detailed synthetic procedure, and characterization of the products by MALDI-TOF MS (Microflex LRF, BrukerDaltonics, Billerica, USA) and ${ }^{1} \mathrm{H}-\mathrm{NMR}$ (Varian Unity INOVA $400 \mathrm{MHz}$, McKinley Scientific, Sparta, NJ, USA)were reported elsewhere (26).

Peptide self-assembly was tested in aqueous solution using different salt $(\mathrm{NaCl})$ concentrations to study the effect of $\mathrm{NaCl}$ concentration on the self-assembly behavior of the designer peptides. Briefly, P1/deionized water solution $(11 \mathrm{mg} / \mathrm{mL})$ and $\mathrm{P} 2 /$ deionized water solution $(11 \mathrm{mg} / \mathrm{mL})$ were prepared by dissolving P1 in deionized water with 0.03 $\mathrm{mM}$ of $\mathrm{NaOH}(\mathrm{pH} 9.0)$ and $\mathrm{P} 2$ in deionized water with $0.03 \mathrm{mMHCl}(\mathrm{pH} 5.4)$. The solutions of P1 $(0.5 \mathrm{~mL})$ and $\mathrm{P} 2(0.5 \mathrm{~mL})$ were combined with magnetic stirring and sonicating for $30 \mathrm{~min}$ to give a uniform mixture, and then the $\mathrm{NaCl}$ concentration in the combined P1/P2 solution was adjusted to $0.03 \mathrm{mM}, 10 \mathrm{mM}$ and $30 \mathrm{mM}$ by adding the appropriate amount of the $\mathrm{NaCl}$ solution $(1 \mathrm{M})$. The combined $\mathrm{P} 1 / \mathrm{P} 2$ solution was allowed to self-assemble for $24 \mathrm{~h}$ at room temperature.

Approximately $10 \mu \mathrm{L}$ of each self-assembled peptide hydrogel was transferred onto the surface of a newly cleaned silicon wafer (cleaned by 10 wt.\% $\mathrm{HCl}$ and 10 wt.\% $\mathrm{NaOH}$ solutions each for $10 \mathrm{~h}$ respectively, and then washed by ethanol and deionized water several times with sonication) and air dried for $1 \mathrm{~h}$ at room temperature. The assembled peptide membrane was rinsed with $100 \mu \mathrm{L}$ of deionized water to remove unattached peptides and salt. The rinsing and washing process was repeated three times, and the specimen was allowed to air dry. Once dry, the peptide-covered wafer was coated with a platinum layer $(10 \mathrm{~nm}$ ) for FESEM characterization (Hitachi S-4700, Hitachi High Technologies America, Inc.).

In addition to testing the peptide self-assembly in aqueous solution at different salt concentrations, the process was studied at different total peptide concentrations of 5.5 
$\mathrm{mg} / \mathrm{mL}$ and $11 \mathrm{mg} / \mathrm{mL}(\mathrm{P} 1: \mathrm{P} 2=1: 1 \mathrm{wt} . / \mathrm{wt}$.$) at a singleNaCl concentration (10 \mathrm{mM})$. The self-assembled peptide gel was prepared on the silicon wafer surface in the same way as before and characterized by FESEM.

\subsubsection{Synthesis of amphiphilictriblock copolymers with reactive terminals by RAFT polymerization}

Amphiphilictriblock copolymers of PVP-b-PMMA-b-PVP and PHEMA-b-PMMA-b-PHEMA, were synthesized by RAFT polymerization usingS, $\mathrm{S}^{\prime}$-bis $\left(\alpha, \alpha^{\prime}\right.$-dimethylacetic acid) trithiocarbonate (BDAT) as chain transfer agent (CTA) and AIBN as initiator. This CTA gave reactive carboxylic acid terminals at both chain ends (HOOC-ABA-COOH). The synthesis of BDAT was described elsewhere (26).

The polymerization was done in two steps to build up the amphiphilic block copolymer. In the first step the hydrophilic block(PVP or PHEMA) was prepared in dioxaneat $70^{\circ} \mathrm{C}(24 \mathrm{~h}$ under nitrogen atmosphere) usingthe following ratio of reagents: $[\mathrm{M}]_{\mathrm{o}}:[\mathrm{BDAT}]_{\mathrm{o}}:[\mathrm{AIBN}]_{\mathrm{o}}=1000: 5: 1$. The product was precipitated in cool diethyl ether to obtain the PVP or PHEMA block as a solid. The precipitation was repeated 3 times to remove any unreacted monomer. The polymer containing the hydrophilic block was vacuum-dried at $50{ }^{\circ} \mathrm{C}$ for $12 \mathrm{~h}$. In the second step, the hydrophilic polymer was used as a macro-CTA to copolymerize with MMA in a dioxane/DMF $(4: 1 \mathrm{v} / \mathrm{v})$ solvent mixture with additional AIBN added $(0.0012 \mathrm{~g})$. The reaction was continued at $70{ }^{\circ} \mathrm{C}(48 \mathrm{~h})$ under nitrogen atmosphere to obtain the amphiphilic triblock copolymer. The as-made copolymer solution was purified by precipitating in cool diethyl ether 3 times to remove unreacted residues and vacuum-dried at $50{ }^{\circ} \mathrm{C}$ for $12 \mathrm{~h}$.

The length of the hydrophilic block was maintained as a constant (with actual $\mathrm{X}_{\mathrm{N}}=$ 78 for PVP and 64 for PHEMA, see Table 3.1) but the length of the PMMA block, synthesized in the second step, was varied to adjust the ratio of the hydrophilic to hydrophobic block in the nanoparticles. This resulted in different core sizes, as well as 
different nanoparticle sizes. The reactant ratios, and molar mass and yields of the copolymer products are summarized in Table 3.1.

Table 3.1. Reactant ratios and products (Macro-CTA and Copolymer) by RAFT.

\begin{tabular}{|c|c|c|c|c|}
\hline Macro-CTA & {$[\mathrm{M}]_{0}:[\mathrm{CTA}]_{0}:[\mathrm{I}]_{0}$} & $\mathbf{X}_{\mathbf{N}^{\mathbf{a}}}$ & $\mathbf{M N}_{\mathbf{N}}^{\mathrm{a}}, \mathbf{D a}$ & $\begin{array}{c}\text { Yield } \\
\%\end{array}$ \\
\hline PVP & $1000: 5: 1$ & 78 & 8954 & 84.0 \\
\hline PHEMA & 1000:5:1 & 64 & 8557 & 92.8 \\
\hline Copolymer & $\begin{array}{c}\text { Mass Ratio } \\
\text { (Macro-CTA )o:(MMA)o }\end{array}$ & $\mathbf{X}_{\mathbf{N}} / \mathbf{X}_{\mathbf{M}}$ & $\mathbf{M}_{\mathbf{N}}^{\mathbf{b}}, \mathbf{D a}$ & $\begin{array}{c}\text { Yield } \\
\%\end{array}$ \\
\hline PVP-b-1.5PMMA-b-PVP & $1: 1.5$ & $78 / 86$ & 17564 & 72.8 \\
\hline PVP-b-3PMMA-b-PVP & $1: 3$ & $78 / 186$ & 27576 & 70.2 \\
\hline PVP-b-6PMMA-b-PVP & $1: 6$ & $78 / 524$ & 61417 & 53.7 \\
\hline PHEMA-b-3PMMA-b-PHEMA & $1: 3$ & $64 / 102$ & 18769 & 65.8 \\
\hline
\end{tabular}

a. $\mathrm{M}_{\mathrm{N}}$ tested by MALDI-TOF; $X_{N}=\frac{M_{N}-M_{B D A T}}{M_{\text {Monomer }}}$.

b. $\mathrm{M}_{\mathrm{N}}$ calculated from ${ }^{1} \mathrm{H}$ NMR by ratio of integrated peaks.

\subsubsection{Characterization of hydrophilic block and tri-block copolymers}

The number average molecular weight $\left(\mathrm{M}_{\mathrm{N}}\right)$ of the hydrophilic block (PVP and PHEMA) was determined by MALDI-TOF MS and the number average degree of polymerization $\left(\mathrm{X}_{\mathrm{N}}\right)$ was calculated from the measured $\mathrm{M}_{\mathrm{N}}$. These results are given in Table 3.1. A detailed analysis of the MALDI-TOF MS spectra is described elsewhere (26). ${ }^{1} \mathrm{H}-\mathrm{NMR}$ spectra (performed in DMSO-d6) and peak assignments are shown in Figure 3.1 (a, b). The integrated peak area from the PVP and PMMA blocks gives the block ratio $\mathrm{X}_{\mathrm{PVP}}$ : $\mathrm{X}_{\mathrm{PMMA}}$ and thus the number average molecular weight of the triblock 
copolymer is determined. For example, analysis of PVP-b-1.5PMMA-b-PVP shows that theintegratedarea of the peak at $\delta: 3.14\left(-\mathrm{C}_{2}-\mathrm{N}\right.$, from PVP block) and the peak areas at

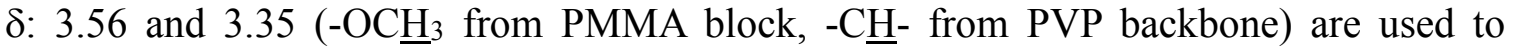
calculate the block ratio of $\mathrm{X}_{\mathrm{PVP}}: \mathrm{X}_{\mathrm{PMMA}}=1: 1.10$ according to equation (1).

$$
\frac{2 X_{P V P}}{3 X_{P M M A}+X_{P V P}}=\frac{\operatorname{Area}_{\left(-\mathrm{CH}_{2}-\mathrm{N}\right)}}{\operatorname{Area}_{\left(-\mathrm{OCH}_{3},-\mathrm{CH}-\right)}}=\frac{11.41}{24.49}
$$

The actual block ratios of other copolymers (PVP-b-3PMMA-b-PVP, PVP-b-6PMMA-b-PVP and PHEMA-b-3PMMA-b-PHEMA) are similarly calculated to be $1: 2.38,1: 6.72$ and 1:1.60. According to the known $X_{N}$ and the actual block ratios calculated, the $\mathrm{X}_{\mathrm{N}}$ and $\mathrm{M}_{\mathrm{N}}$ of tri-block copolymers are thus determined and listed in

\section{Table 3.1.}

The peak assignments for the ${ }^{1} \mathrm{H}-\mathrm{NMR}$ spectra of copolymers were made as shown below. PVP-b-PMMA-b-PVP (in DMSO-d6, $\delta$ ): 0.73-1.04 (- $\left.\underline{\mathrm{C}}_{3}\right), 1.40-1.82\left(-\mathrm{C}_{2}-\right)$,

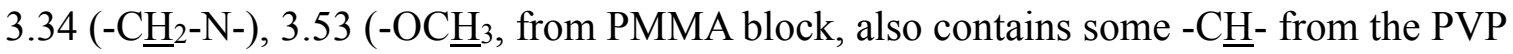

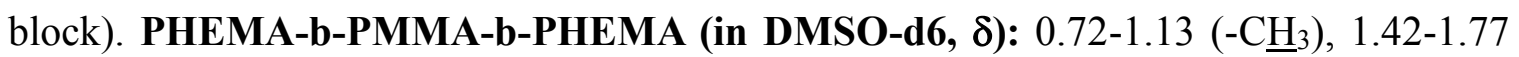
$\left(-\mathrm{CH}_{2}-\right), 3.53\left(-\mathrm{OC}_{3}\right.$, from PMMA block, also contains $-\underline{\mathrm{C}}_{2}-\mathrm{OH}$ from the PHEMA block), $3.87\left(-\mathrm{CH}_{2}-\mathrm{O}-\mathrm{C}=\mathrm{O}\right)$.

\subsubsection{Coupling reaction of copolymer with $\mathrm{P} 1$ and $\mathrm{P} 2$}

The synthesized peptides (P1 and P2) were coupled with the desired amphiphilic copolymer to form peptide-copolymer conjugates. The coupling reaction was performed betweenthe carboxylic acid terminals of the desiredpolymer and the amine terminalsof the desired peptide using 2-(7-aza-1-H-benzotriazol-1-yl)-1,1,3,3-tetramethylaminium (HATU) as the coupling agent, as shown in Scheme 3.1. The process for the PVP-b-6PMMA-b-PVP system is described in greater detail as an example. The copolymer $\left(1.0 \mathrm{~g}, \sim 1.6 \times 10^{-5} \mathrm{~mol}\right)$ was dissolved in DIPEA/DMF $(8.0 \mathrm{~mL}, 0.9 \mathrm{M}$ DIPEA 
in $\mathrm{DMF}$ ) at room temperature in a $25 \mathrm{~mL}$ three neck round bottom flask with magnetic stirring. The reaction solution was degassed for 15 min with nitrogen and then HATU $\left(0.012 \mathrm{~g}, \sim 3.2 \times 10^{-5} \mathrm{~mol}\right)$ was added to activate the carboxylic acid groups. P1 (0.10 $\mathrm{g}$, $\left.8.2 \times 10^{-5} \mathrm{~mol}\right)$ was pre-dissolved in DMF $(5.0 \mathrm{~mL})$ in a glass vial, and then transferred into the activated copolymer solution via syringe. The reaction was continued for $45 \mathrm{~min}$ at room temperature with magnetic stirring and nitrogen gas bubbling through solution. P2 was similarly coupled with a second batch of copolymer. The peptide-copolymer conjugate was precipitated in cool diethyl ether and centrifuged to remove un-reacted chemicals. The precipitation was repeated 3 times to obtain purified conjugate solids, and dried under reduced pressure for $12 \mathrm{~h}$ at $50{ }^{\circ} \mathrm{C}$ before storing in a sealed vessel in the refrigerator at $4{ }^{\circ} \mathrm{C}$.

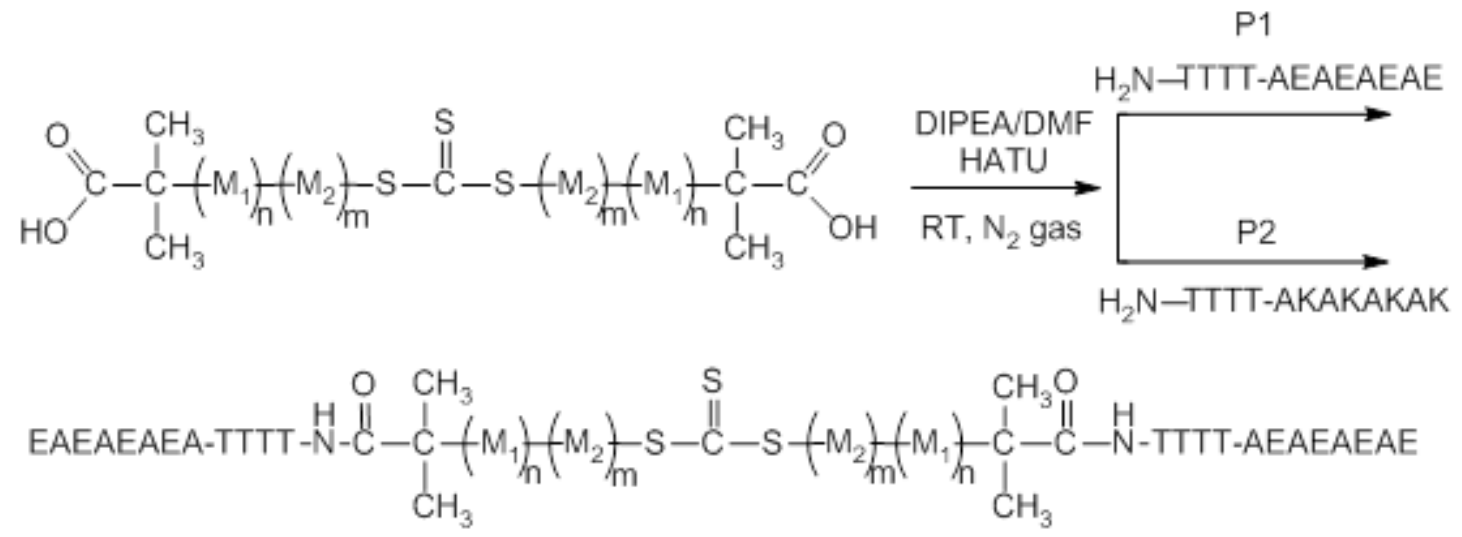

a. P1-copolymer conjugates

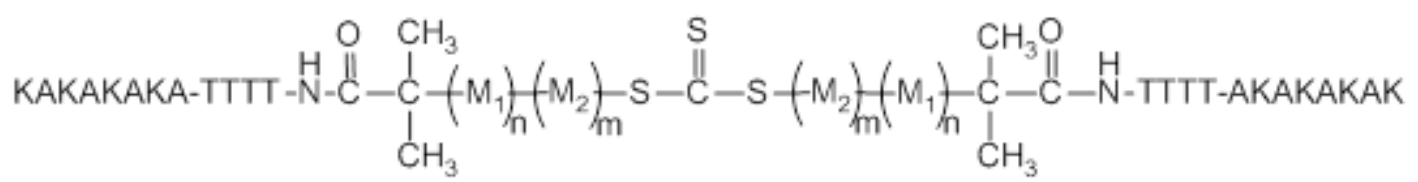

b. P2-copolymer conjugates

Scheme 3.1. Synthesis of peptide-copolymer conjugates by coupling reaction of peptide amine terminal with copolymer carboxylic acid terminals. 
Other copolymers that possessed different block ratios were coupled with P1 and P2 using the same procedure. The peptide-copolymer conjugates were characterized by FT-IR (Spectrum One, Perkin Elmer, Massachusetts, USA) to verify the coupling reaction. The sample designationsfor the peptide-functionalized copolymer conjugates are shown in Table 3.2.

\subsubsection{Self-assembly study of peptide-copolymer conjugates in aqueous solution}

All peptide-copolymer conjugates were first self-assembled in aqueous solution to form the peptide-functionalized nanoparticles (P1-NP-P1 and P2-NP-P2). Then the individual nanoparticles bearing complementary peptides (P1-NP-P1 and P2-NP-P2) were combined together where they then assembled into 1D nanoparticle fibers and eventually formed 3D scaffolds as directed by the ionic complementary assembly between $\mathrm{P} 1$ and $\mathrm{P} 2$.

The assembly process of the nanoparticles into nanoparticle fibers was accomplished in three steps as follows. First the P1-copolymer conjugates $(20 \mathrm{mg})$ were dissolved in DMSO $(2 \mathrm{~mL})$ to give a clear precursor solution, and then the precursor solution was slowly injected $(0.4 \mathrm{~mL} / \mathrm{min})$ into weakly basic deionized water $(10 \mathrm{~mL}, \mathrm{pH} 9.0$, adjusted by $1 \mathrm{M} \mathrm{NaOH}$ solution) via a $31 \mathrm{G}$ syringe while being stirred at $600 \mathrm{rpm}$ by a magnetic stirrer to self-assemble into a P1-NP-P1 suspension. After completing the injection of the precursor solution, the magnetic stirring was continued for $30 \mathrm{~min}$ and then sonicated 2 min to form a stable peptide-nanoparticle suspension. In the second step, the $\mathrm{P} 2$-copolymer conjugates were first solvated in DMSO to form a precursor solution and then added into weakly acidic deionized water $(10 \mathrm{~mL}$, pH 5.4, adjusted by $1 \mathrm{M} \mathrm{HCl})$ to form a P2-NP-P2 suspension and sonicated for $2 \mathrm{~min}$.

The purpose of forming the nanoparticle suspensions in weakly basic ( $\mathrm{pH} 9.0)$ and weakly acidic ( $\mathrm{pH}$ 5.4) deionized water was to completely ionize the carboxylic acid side groups from $\mathrm{P} 1$ and amine side groups from $\mathrm{P} 2$ to bear negative and positive charges 
respectively. This gave two separate suspensions of nanoparticles bearing complementary peptides that were self-repulsive ensuring no assembly until the two separate suspensions are combined.

Table 3.2. Hydrodynamic diameter of the peptide-copolymer conjugate nanoparticles by DLS and self-assembly time for each nanoparticle pair.

\begin{tabular}{lllc}
\hline Peptide-Copolymer Conjugate & $\begin{array}{c}\text { Assembled } \\
\text { NP name }\end{array}$ & $\begin{array}{c}\text { NP Size } \\
\text { (nm) }\end{array}$ & $\begin{array}{c}\text { Assembly } \\
\text { time (h) }\end{array}$ \\
\hline P1-PVP-b-1.5PMMA-b-PVP-P1 & P1-NP1-P1 & 178 \pm 72 & 3 \\
P2-PVP-b-1.5PMMA-b-PVP-P2 & P2-NP1-P2 & & 6 \\
\hline P1-PVP-b-3PMMA-b-PVP-P1 & P1-NP2-P1 & $227 \pm 97$ & 10 \\
P2-PVP-b-3PMMA-b-PVP-P2 & P2-NP2-P2 & \\
\hline P1-PVP-b-6PMMA-b-PVP-P1 & P1-NP3-P1 & $580 \pm 264$ & 6 \\
P2-PVP-b-6PMMA-b-PVP-P2 & P2-NP3-P2 & & \\
\hline P1-PHEMA-b-3PMMA-b-PHEMA-P1 & P1-NP4-P1 & & \\
P2-PHEMA-b-3PMMA-b-PHEMA-P2 & P2-NP4-P2 & & \\
\hline
\end{tabular}

Table 3.2 shows the hydrodynamic diameter of the individual nanoparticle-peptide conjugates before scaffold assembly, and the time allowed for scaffold assembly after the complementary nanoparticle conjugates are combined. When larger nanoparticle conjugates were used the allowed assembly time was increased. For example, the stable P1-NP1-P1 suspension was injected into the stable P2-NP1-P2 suspension with gentle magnetic stirring at $400 \mathrm{rpm}$ for $30 \mathrm{~min}$ to give a uniformly mixed suspension. Within 10 minutes after the stirring was discontinued there was visual evidence of assembly with the appearance of a 'sponge-like' phase that was settling at the bottom of the vial. The allowed assembly time ranged from 3-10 h, with longer times being allowed for the larger nanoparticles. 
The self-assembled nanoparticle scaffolds from smaller peptide-nanoparticles (from P1-NP1-P1 and P2-NP1-P2) and larger peptide-nanoparticles (from P1-NP3-P1 and P2-NP3-P2) were applied to silicon wafers to allow observation by FESEM. Briefly, the assembled nanoparticles $(100 \mu \mathrm{L})$ were transferred onto a cleaned silicon wafer surface (cleaned by $10 \mathrm{wt} . \% \mathrm{HCl}$ solution and $10 \mathrm{wt} . \% \mathrm{NaOH}$ solution for $10 \mathrm{~h}$ respectively, and then washed by ethanol and deionized water several times with sonication). The nanoparticle layer on silicon wafer surface was air dried to leave an assembled nanoparticle membrane (designated as a $2 \mathrm{D}$ scaffold) and then vacuum dried at $50{ }^{\circ} \mathrm{C}$ for $6 \mathrm{~h}$. The nanoparticle membrane was coated with platinum $(\mathrm{Pt}, \sim 5 \mathrm{~nm})$ prior to FESEM characterization.

\subsubsection{Self-assembly study of peptide-functionalized nanoparticles with additional peptides}

A self-assembly study of the peptide-functionalized nanoparticles was performed in the presence of excess"free" peptides (P1 and P2) to determine if their presence effected the porous structure and mechanical stability of the final 3D nanoparticle scaffolds. P1-NP3-P1 and P2-NP3-P2 suspensions (Table 3.2) were similarly prepared in deionized water as described before, and the nanoparticle suspensions were dialyzed $24 \mathrm{~h}$ to remove organic solvent in $2 \mathrm{~L}$ of fresh weak basic deionized water ( $\mathrm{pH} 9.0$ for P1-NP3-P1) and weak acidic deionized water ( $\mathrm{pH} 5.4$ for P2-NP3-P2) with replacing fresh deionized water every $12 \mathrm{~h}$. The concentration of both purified nanoparticle suspensions was gravimetrically determined to be $2.6 \mathrm{mg} / \mathrm{mL}$.

Then $0.5 \mathrm{~mL}$ of P1-NP3-P1 suspension was well mixed with $0.5 \mathrm{~mL}$ of P1 solution (11 $\mathrm{mg} / \mathrm{mL}$ in deionized water with $\mathrm{pH}$ at 9.0 ) to form a mixed suspension of P1-NP3-P1/P1 (1.3 mg/mL of nanoparticle with $5.5 \mathrm{mg} / \mathrm{mL}$ of P1). A suspension of P2-NP3-P2/P2 (same concentration as P1-NP3-P1/P1) was similarly prepared. Then the P1-NP3-P1/P1 suspension was combined with the P2-NP3-P2/P2 suspension by magnetic 
stirring at $400 \mathrm{rpm}$ for $2 \mathrm{~h}$, and allowed to settle during self-assembly to form nanoparticle/peptide co-assembled composite hydrogel after adjusting $\mathrm{NaCl}$ concentration to be $10 \mathrm{mM}$ by adding $1 \mathrm{M} \mathrm{NaCl}$ solution. The concentration of the nanoparticle/peptide composite hydrogel was $1.3 \mathrm{mg} / \mathrm{mL}$ of nanoparticle with $5.5 \mathrm{mg} / \mathrm{mL}$ of total peptides (P1 and P2). Using same procedure, $1.3 \mathrm{mg} / \mathrm{mL}$ of nanoparticle with 11 $\mathrm{mg} / \mathrm{mL}$ and $22 \mathrm{mg} / \mathrm{mL}$ of total peptides were prepared to compare their stability and porous structure of 3D nanoparticle/peptide hydrogel scaffolds.

$10 \mu \mathrm{L}$ of the self-assembled nanoparticle/peptide composite hydrogel was dropped onto a cleaned silicon wafer surface to air dry, giving2Dnanoparticle/peptide membranes. The membrane was then rinsed with $100 \mu \mathrm{L}$ of fresh deionized water 3 times to remove un-bonded peptides and salts and again allowed to air dry. Another $10 \mu \mathrm{L}$ of the composite hydrogel $(1.3 \mathrm{mg} / \mathrm{mL}$ nanoparticle with $22 \mathrm{mg} / \mathrm{mL} \mathrm{P1}$ and P2) was placed in a silicon wafer and dried under reduced pressure at room temperaturewithout washing with deionized water in order to compare the $3 \mathrm{D}$ morphologies of the co-assemblednanoparticle/peptide scaffold because vacuum drying can quickly remove solvent to retain a $3 \mathrm{D}$ structure, but air drying only gives a compact $2 \mathrm{D}$ membrane. Allsamples were then coated with a layer of platinum $(5 \mathrm{~nm})$ for FESEM characterization.

\subsubsection{Controlled release test with hydrophobic fluorescein as model drug}

A controlled release study was performed for 23 days in deionized water using 4',5'-dibromofluorescein (DBF) as model for a small hydrophobic drug. The peptide-copolymer conjugates together with the DBF were dissolved in DMSO to form two host solutions, DBF/P1-PVP-b-1.5PMMA-b-PVP-P1 (38 mg +0.2 wt.\% $\mathrm{DBF}) / \mathrm{DMSO}(4 \mathrm{~mL})$ solution and DBF/P2-PVP-b-1.5PMMA-b-PVP-P2 (38 mg +0.2 wt.\% DBF)/DMSO (4mL) solution. The DBF/P1-copolymer host solution was then 
slowly injected into weakly basic deionized water $(10 \mathrm{~mL}, \mathrm{pH} 9.0)$ to give a self-assembled DBF-loaded P1-NP1-P1 suspension. The DBF/P2-copolymer host solution was similarly injected into weakly acidic deionized water $(10 \mathrm{~mL}, \mathrm{pH} 5.4)$ to give a self-assembled DBF-loaded P2-NP1-P2 suspension. The DBF-loaded P1-NP1-P1 suspension was then injected (23G syringe) into the DBF-loaded P2-NP1-P2 suspension with gentle magnetic stirring at $400 \mathrm{rpm}$ for $1 \mathrm{~h}$ to give a uniform mixture. The mixed nanoparticle suspension was allowed to self-assemble into a3Dnanoparticle scaffold at the bottom of the vial.

The concentration of the DBF-loaded nanoparticle scaffold suspension was gravimetrically determined. This was done bygently shaking the suspensionto gain a uniform dispersion, and then $2.0 \mathrm{~mL}$ of the suspension was measured and gently heated to remove solvent to obtain a dimensionally stable dry mass $(7.0 \mathrm{mg})$. The remainder of the nanoparticle suspension was then centrifuged to isolate the uncaptured DBF in the supernatant. The nanoparticle scaffoldsolids were also collected and washed with fresh deionized water $(2.0 \mathrm{~mL}$ each time $)$ and centrifuged at $4000 \mathrm{rpm}$ for $10 \mathrm{~min}$ to isolateany additionaluncaptured DBFthat had been adsorbed onto the nanoparticle surface. This washing and centrifuging process was repeated two additional times, and all the supernantants were combined to aid in collecting all uncaptured DBF. The combined supernatant was evaporated in a dark environment, and the isolated DBF was then dissolved in a solution of deionized water/ethanol $(1: 1, \mathrm{v} / \mathrm{v})$ and transferred into a volumetric flask to allow the uncaptured DBF to be quantified by fluorescence spectrometry. Thewashed DBF-loaded nanoparticle solids were then dispersed in $3.0 \mathrm{~mL}$ of fresh deionized water and set up at $37 \pm 1{ }^{\circ} \mathrm{C}$ for a controlled release test. At each time interval, the released DBFsolution was isolated from the solid nanoparticles by centrifugation at $4000 \mathrm{rpm}$ for $10 \mathrm{~min}$, and then the DBF-containing supernatant was collected for testing. Then $3.0 \mathrm{~mL}$ of fresh deionized water was added to replace the 
collected fluid and thecontrolled release test on the nanoparticles was continued. An equivalent volume of ethanol was added to each liquid aliquot collected from the sample being tested so that the releasedDBFwas in a 1:1 water/ethanol solution foranalysis.

Separately, a series of standard DBF solutionswere prepared using deionized water/ethanol $(1: 1, \mathrm{v} / \mathrm{v})$ to givea standard curve to quantify uncaptured DBF and released DBF from the nanoparticle scaffolds. The uncapturedDBFsolution, the released DBFsample solution, and standard DBF solution, were analyzed by fluorescence spectrometer (SPEX FLUOROLOG equipped with SPEX $16810.22 \mathrm{~m}$ Spectrometer and SPEX dM 3000 power controller, HORIBA Scientific, USA) with slit setup at $0.50 \mathrm{~mm}$ and $\lambda_{\text {emission }}$ intensity collected at $532 \mathrm{~nm}$. The concentration of uncapturedDBFand each released DBFsample were quantitatively determined according to the standard curve and Beer's Law (Figure 3.1S, supplementary data). And the actual loading content (wt. \%) of DBF in nanoparticles was calculated based on equation (2).

$$
\text { Actual } \quad w t . \%=\frac{W_{\text {intial drug }}-W_{\text {uncaptured drug }}}{W_{\text {drug-loaded nanopartile }}} \times 100 \%
$$

All other peptide-copolymer conjugates were loaded with model drug of DBF using the same procedure, and the actual loading content (wt. \%) was determined. The results are shown in Table 3.3. The cumulative release of DBF (wt.\%) based on initially loaded DBF amount was calculated and results are shown in Figure 3.10. 
Table 3.3. Loading efficiency of model drug (DBF) in different nanoparticles.

DBF

\begin{tabular}{|c|c|c|c|c|}
\hline & Scaffold/NPs & Theo. Loading & Act. Loading & Loading \\
\hline & & Wt. $\%$ & Wt. \% & Efficiency\% \\
\hline & P1-NP1-P1 & & & \\
\hline S-1 & P2-NP1-P2 & 0.20 & 0.19 & 95 \\
\hline & P1-NP2-P1 & & & \\
\hline S-2 & & 0.20 & 0.16 & 80 \\
\hline & P2-NP2-P2 & & & \\
\hline & P1-NP3-P1 & 020 & 010 & 05 \\
\hline ד & P2-NP3-P2 & 0.20 & 0.13 & בני \\
\hline$C_{1}{ }_{1}$ & P1-NP4-P1 & & & \\
\hline דים & P2-NP4-P2 & 0.20 & 0.10 & 30 \\
\hline
\end{tabular}

\subsubsection{Multiple-drug loading and controlled release test using nitrofurazone and amoxicillin as model drugs}

Controlled release studies were also done to test the simultaneous release of multiple drugs contained within one self-assembled scaffold system. These tests used nitrofurazone as a moderately hydrophobic model drug and amoxicillin asahydrophilic model drug. P1-NP-P1 and P2-NP-P2 were separately loaded with 5.0 wt.\% of nitrofurazone and $5.0 \mathrm{wt} . \%$ of amoxicillin respectively using the same procedure as for DBF loading.

The two drug-loaded nanoparticle suspensions (P1-NP-P1 and P2-NP-P2) were then mixed together to self-assemble into a two-drug loaded nanoparticle scaffold. The nanoparticle scaffold concentration, uncaptured drug quantities and controlled release tests were performed using the procedures described for DBF, except the controlled release test time wasonly $96 \mathrm{~h}$ due to the rapid release of the hydrophilic model drug, 
amoxicillin. The amount of uncaptured and released drugs was quantified using an HPLC equipped with a UV-vis detector (SPD-20AT/SPD-20A, SHIMADZU Corporation, Kyoto, Japan). An example of calibration curve of nitrofurazone and amoxicillin for quantitative analysis is shown in Figure 3.2S a,b (supplementary data). The actual quantity of nitrofurazone and amoxicillin that were loaded into the nanoparticles is given in Table 3.4. The cumulative release of both drugs (wt.\% based on initially loaded amount) was determined and these results are shown in Figure 3.11.

Table 3.4. Loading efficiency of model drugs (Nitrofurazone and Amoxicillin) in different nanoparticles.

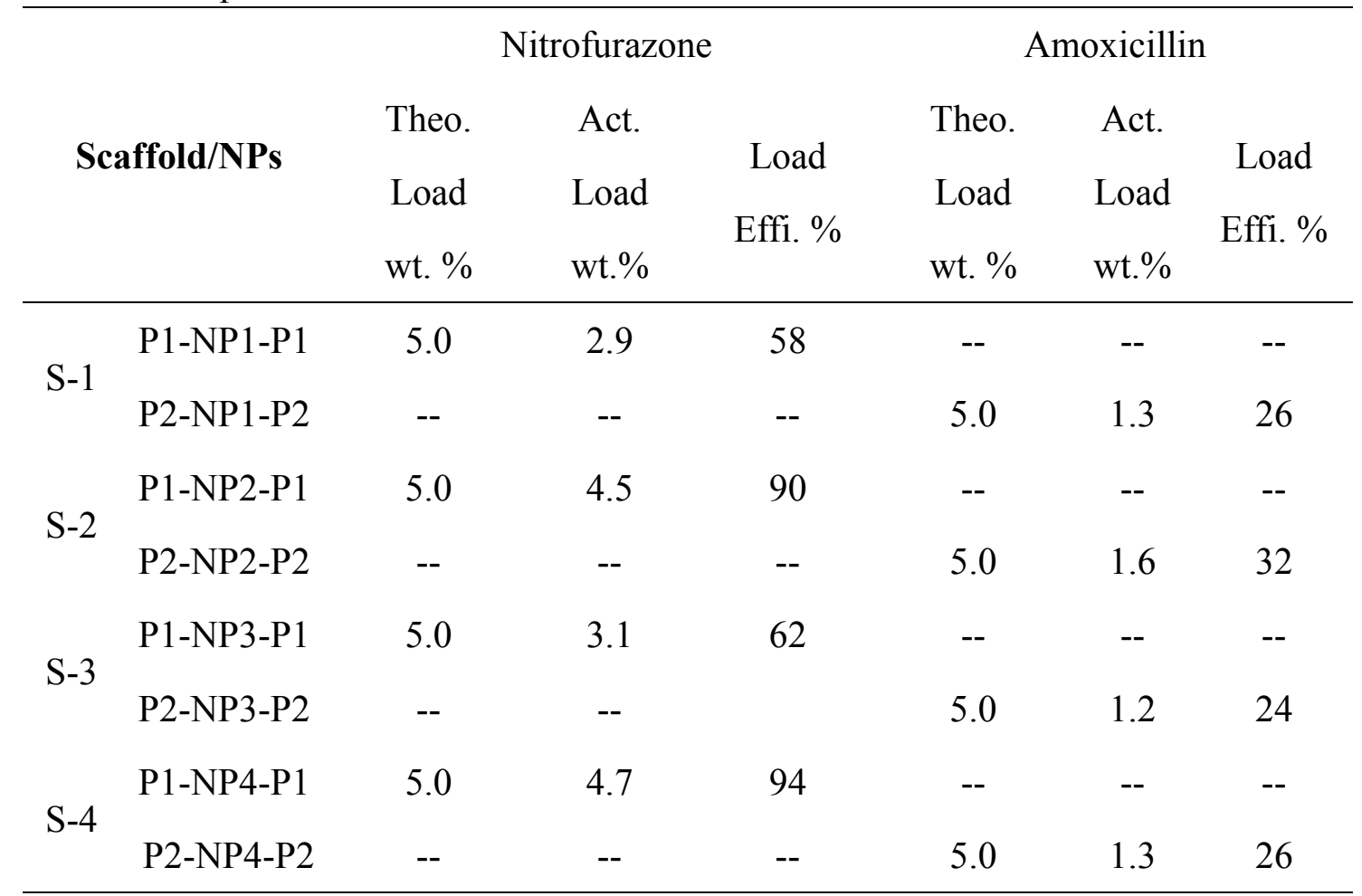

\subsubsection{Cytotoxicity test on NIH3T3 cell lines}

Nanoparticle scaffolds that were self-assembled from P1-NP(x)-P1 and P2-NP(x)-P2 (Table 3.2, NP(x) represents NP1, NP2, NP 3, or NP4), possessing different particle sizes and/or compositions, were tested for biocompatibility with NIH3T3 fibroblast cells. This 
testing was done by incubating NIH3T3 fibroblast cellsin a glass culture dish that already possessed an assembled 2D scaffoldingmembraneon the bottom of the dish. The study was done to test the effects of the different scaffolds on cell viability and activity.

Each self-assembled nanoparticle scaffold (Table 3.3, S-1 to S-4) was similarly prepared using the same self-assembly procedure. For example, the P1-NP1-P1 suspension was self-assembled from P1-PVP-b-1.5PMMA-b-PVP-P1/DMSO (40 $\mathrm{mg} / 4 \mathrm{~mL})$ precursor solution in weakly basic deionized water $(10 \mathrm{~mL}, \mathrm{pH} 9.0)$, and the P2-NP1-P2 suspension was prepared from P2-PVP-b-1.5PMMA-b-PVP-P2/DMSO (40 $\mathrm{mg} / 4 \mathrm{~mL})$ precursor solution but in weakly acidic deionized water $(10 \mathrm{~mL}, \mathrm{pH} 5.4)$. The two suspensions were then coupled together with gentle magnetic stirring at $400 \mathrm{rpm}$ for $30 \mathrm{~min}$ and settled for $3 \mathrm{~h}$ to obtain scaffold sample S-1. Other scaffold samples (S-2 to S-4) were prepared using the same method, but longer assembly time was allowed for bigger nanoparticles. All scaffold samples were dialyzed using a dialysis tube (Nominal MWCO 3,500, from Fisher Scientific) in PBS solution for $48 \mathrm{~h}$ to remove organic solvent and free peptide (P1 and P2). Fresh PBS (1L for each sample) was replaced for dialysis at every $24 \mathrm{~h}$ interval. The purified nanoparticle scaffold was then transferred into a centrifuge tube $(15 \mathrm{~mL})$ and stored at $4{ }^{\circ} \mathrm{C}$ for application.

The concentration of the purified scaffold was gravimetrically determined. $2.0 \mathrm{~mL}$ of uniformly dispersed scaffold suspension (S-1) was measured and gently heated to remove solvent, leaving dry mass of $7.2 \mathrm{mg}$ after taking off the background mass of $2.0 \mathrm{~mL}$ of dried PBS. So the scaffold concentration of S-1 was determined to be $3.5 \mathrm{mg} / \mathrm{mL}$. Other nanoparticle scaffold samples (S-2, S-3 and S-4) were determined to be 2.9, 3.0, and 3.6 $\mathrm{mg} / \mathrm{mL}$ respectively.

Based on the known concentrations, approximately $500 \mu \mathrm{g}$ of dry scaffold mass were transferred into each pre-sterilized glass culture dish. The suspension drop was air dried 
at room temperature overnight, leaving an assembled scaffold membrane (a circle layer with diameter of $\sim 2 \mathrm{~cm}$ ) on the dish bottom.

Fibroblast cells were cultured in cell media and passaged at $\sim 95 \%$ confluency so that the cells were able to be cultured for extended periods of time. Cell concentration was determined to be $1.5 \times 10^{5}$ cells $/ \mathrm{mL}$ by cytometry. To each culture dish containing $\mathrm{S}-1$ to S-4scaffold membrane, fibroblast cell seeds $\left(\sim 1.5 \times 10^{5}\right.$ cells $)$ were transferred onto the membrane surface and incubated for $\sim 15 \mathrm{~min}$ at $37{ }^{\circ} \mathrm{Cwith} 5 \% \quad \mathrm{CO}_{2}$ in a humidified incubator, and then $5 \mathrm{~mL}$ cell media was slowly added to avoid directly flushing onto the membrane. The culture dish was then incubated at $37{ }^{\circ} \mathrm{C}$ with $5 \% \mathrm{CO}_{2}$ in the incubator and media was refreshed after $12 \mathrm{~h}$ and then replaced every $24 \mathrm{~h}$. The cell growth status was observed every $24 \mathrm{~h}$ using optical microscopy (Nikon Eclipse Ti, Nikon Instruments Inc., USA) and the incubation was allowed to proceedfor 7 days.

In order to improve cell adhesion ability, cell adhesion peptides (RGDS, 1 wt.\% based on peptide-copolymer conjugates) were physically encapsulated into nanoparticles using the same procedure that was used to load the model drugs, and similarly purified by dialysis in fresh PBS.The RGDS-loaded nanoparticle scaffold membrane was similarly prepared on a glass dish, air dried and then incubated with fibroblast cells using same procedure. Both cell proliferation and migration were observed every $24 \mathrm{~h}$ by optical microscopy. 


\subsection{Results and Discussion}

\subsubsection{Rationale for peptide design and study of peptide self-assembly}

The purpose of this project was to design a new type of self-assembled polymeric nanoparticle scaffold with far greater versatility than other scaffolding systems, including multi-drug loading abilities and the ability to vary mechanical strength of the scaffolding, for tissue engineering. The methodology is outlined in Figure 3.1, which demonstrates the use of 2 different nanoparticles assembled into an alternating pattern. The ends of two batches of amphiphilic copolymers are separately functionalized with the our designed peptides (P1 and P2) to form peptide-copolymer conjugates, one carrying negative charges from P1, and the other carrying positive charges from P2. These peptide-copolymer conjugates undergo two-levels of self-assembly in aqueous solution. The first level is the assembly of the core-shell nanoparticle itself, which would be done separately. In principle any biologically acceptable composition could be selected for a desired drug to control its release and a desired nanoparticle size (influenced by the ratio of hydrophilic to hydrophobic monomers and polymer molecular weight) and a desired mechanical strength (influenced by the same variables). For best control a single drug is incorporated into a single nanoparticle, and the numbers of each nanoparticle type would control the total "dose" of each drug. Once the drugs are the appropriate batches of peptide-functionalized nanoparticles are combined to self-assemble into nanoparticle fibers directed by ionic complementary assembly between P1 and P2, and finally, depending on the conditions and assembly time allowed, form 3D nanoparticle scaffolds with multi-drug loading.

As already described, the advantages of this nanoparticle self-assembly approach includes: (1)The ability to incorporate multiple drugs, regardless of that drug's hydrophilicity or hydrophobicity, within the scaffolding; (2)The ability to control the distribution of the different drugs within the scaffold; (3) The ability to separately control 
the release of each drug from a nanoparticle whose composition can be designed for that specific drug; (4)The ability to adjust the porosity and mechanical strength of the final hydrogel; and (5) The ability to introduce this system into a patient by injection followed by controlled self-assembled of the scaffold in situ.

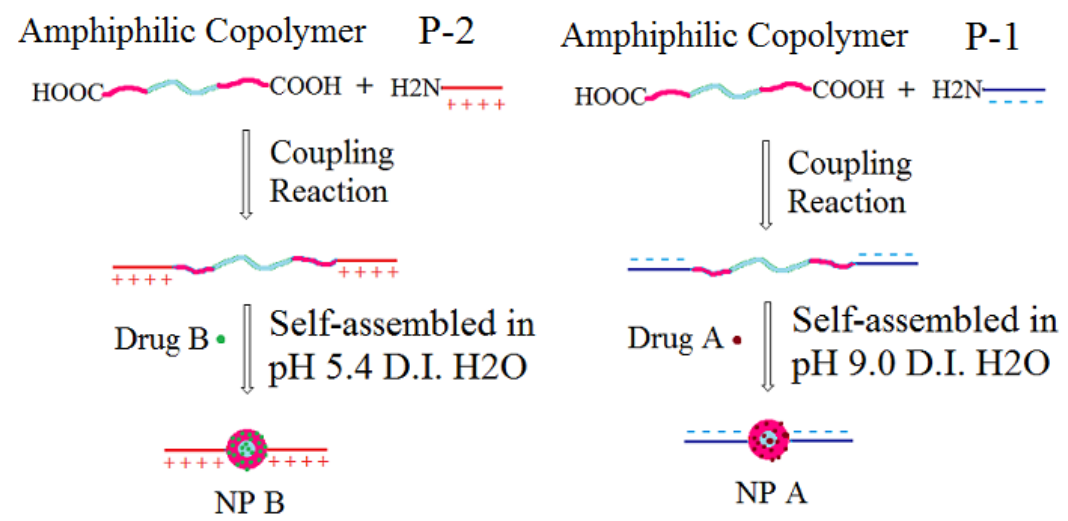

NP A coupled with NP B

by peptide-directed assembly

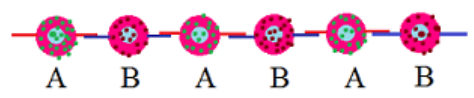

Figure 3.1. General procedure shows fabrication of polymeric nanoparticle scaffold with multi-drug loading abilities.

The key step to achieve these features is the two designed ionic complementary peptides (P1 and P2) with opposite charges that can assemble into $\beta$-sheets in aqueous solution with appropriate $\mathrm{pH}$ range. The two peptides were synthesized by semi-automatic solid phase peptide synthesis strategy with sequence of $\mathrm{H}_{2} \mathrm{~N}$-TTTT-AEAEAEAE-amide (P1) and $\mathrm{H}_{2} \mathrm{~N}$-TTTT-AKAKAKAK-amide (P2). The spacer part (TTTT) is designed to decrease the steric hindrance of copolymer domain to the assembly units (AEAEAEAE for P1 and AKAKAKAK for P2) and thus increase the mobility of the assembly units, and also to increase the solubility of the peptides in aqueous solution. 
The synthesized peptides were characterized by MALDI-TOF MS, ${ }^{1} \mathrm{H}-\mathrm{NMR}$ to confirm the synthesized sequence and chemical structure matching well with theoretical ones. It was also demonstrated that P1 and P2 in PBS/DMF $(1: 1 \mathrm{v} / \mathrm{v})$ at total peptide concentration of $11 \mathrm{mg} / \mathrm{mL}$ showed self-repulsive but mutually attractive self-assembly behavior. All of the work was concretely presented in previous report (26).

Considering biological applications of the self-assembled scaffold, here we further study the self-repulsive but mutually attractive property of the designer peptides (P1 and P2) in aqueous solution with different salt $(\mathrm{NaCl})$ concentration, because it was reported that alkaline salt concentration could significantly affect ionic peptide self-assembly behavior by forming ion-pair bridge between opposite charges, or mask the ion-pair interaction if excessive cation/anion existed (27).

Here, the self-assembly of P1 and P2 was conducted by dissolving P1 in sterile deionized water ( $\mathrm{pH} 9.0$, adjusted by $1 \mathrm{M} \mathrm{NaOH}$ solution) and $\mathrm{P} 2$ in sterile deionized water ( $\mathrm{pH} 5.4$, adjusted by $1 \mathrm{M} \mathrm{HCl}$ solution). Each peptide forms clear aqueous solution with excellent solubility more than $22 \mathrm{mg} / \mathrm{mL}$, but when the two peptide solutions are combined together, the self-assembly is triggered to form hydrogel after settling for $3 \mathrm{~h}$ even the concentration is as low as $5.5 \mathrm{mg} / \mathrm{mL}$. The results are shown in Figure 3.2.

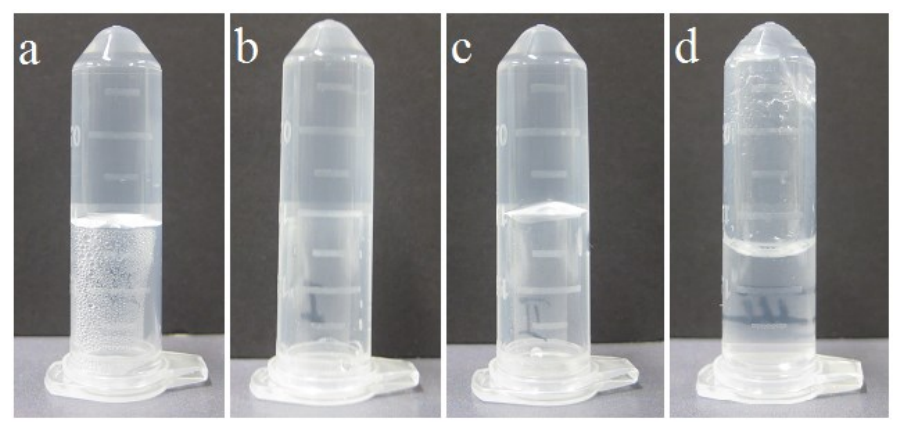

Figure 3.2. Gels formed from self-assembly of P1 and P2 (1:1 wt./wt.) at different total peptide concentration and salt concentration. (a) Gel of $\mathrm{P} 1+\mathrm{P} 2$ at $5.5 \mathrm{mg} / \mathrm{mL}$ with $\mathrm{NaCl}$ concentration of $10 \mathrm{mM}$. (b) Gel of $\mathrm{P} 1+\mathrm{P} 2$ at $11 \mathrm{mg} / \mathrm{mL}$ with $\mathrm{NaCl}$ concentration of 0.03 
mM. (c) Gel of P1+P2 at $11 \mathrm{mg} / \mathrm{mL}$ with $\mathrm{NaCl}$ concentration of $10 \mathrm{mM}$. (d) Gel of $\mathrm{P} 1+\mathrm{P} 2$ at $11 \mathrm{mg} / \mathrm{mL}$ with $\mathrm{NaCl}$ concentration of $30 \mathrm{mM}$.

The gel formation was compared at different $\mathrm{NaCl}$ concentration, which showed that $\mathrm{P} 1$ self-assembles with $\mathrm{P} 2$ most quickly at a10 $\mathrm{mMNaCl}$ concentration, but the gel is weakened when the $\mathrm{NaCl}$ concentration increases to $30 \mathrm{mM}$ (Fig. 3.2d). A self-assembled peptide gel, at a total concentrationofpeptide of $11 \mathrm{mg} / \mathrm{mL}(\mathrm{P} 1+\mathrm{P} 2,1: 1 \mathrm{wt} . / \mathrm{wt}$.) and with $\mathrm{NaCl}$ concentration of $10 \mathrm{mM}$, was dropped on a silicon wafer surface and air dried to form a membrane, that was then coated with $\mathrm{Pt} / \mathrm{Pd}$ layer $(5 \mathrm{~nm})$, was observed by FESEM. The images are shown in Figure 3.3.

Figure 3.3 shows the snowflake-like morphology on top with some smooth membrane on bottom of the self-assembled peptide membrane before rinsing with deionized water. But after rinsing with deionized water to remove the salt, the self-assembled morphology was converted to amorphous aggregation (Fig. 3.3b). This change is mainly due to the high water solubility of the designer peptides (P1 and P2). The high water solubility of peptides usually leads to unstable self-assembled structure and requires high critical concentration to form nanofiber structure $(\mathbf{1 7 , 1 8})$. This appears to be the main reason for our designer peptides that only show certain extent of assembly structure but not very stable nanofiber structure due to its high water solubility and short assembly units (only 8 repeat units, P1: AEAEAEAE and P2: AKAKAKAK). 


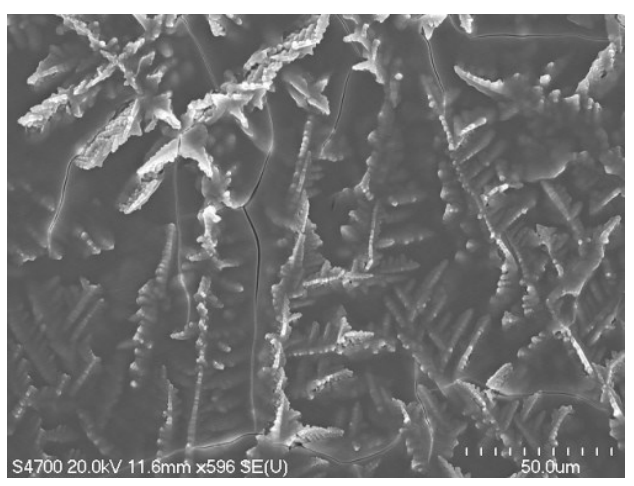

(a)

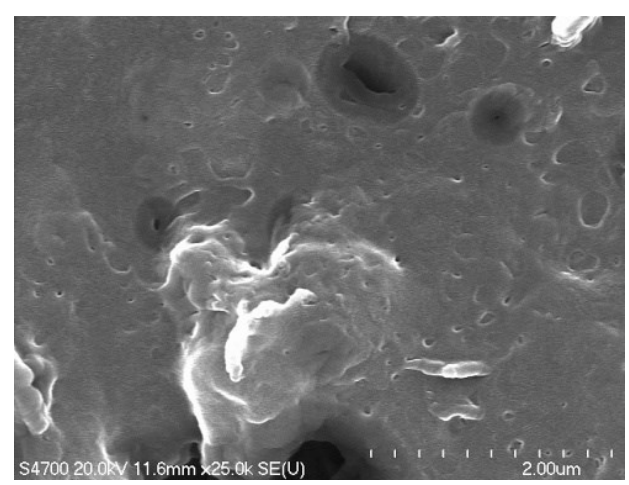

(b)

Figure 3.3. FESEM micrograph of self-assembled peptide membrane of $\mathrm{P} 1+\mathrm{P} 2$ (no nanoparticle conjugates are present) at a total concentration of $11 \mathrm{mg} / \mathrm{mL}$ with $\mathrm{NaCl} 10$ mM. (a) Membrane without rinsing with deionized water. (b) Membrane rinsing with deionized water.

\subsubsection{Synthesis of amphiphilic copolymer with different ratio of hydrophilic and hydrophobic blocks}

In previous work we synthesized peptide-functionalized amphiphilic copolymer (PVP-b-12PMMA-b-PVP or PHEMA-b-12PMMA-b-PHEMA, hydrophilic to hydrophobic block ratio=1:12:1) to self-assemble into peptide-funcitonalized polymeric microparticle (P1-NP-P1 and P2-NP-P2) with a hydrodynamic size of $\sim 1 \mu \mathrm{m}$, to study their self-assembly behavior in aqueous solution to form 1D nanoparticle fibers and 3D scaffolds directed by ionic complementary interaction between P1 and P2 (26). The present work continues that study, and expands its goals to better understand the effect of nanoparticle size on self-assembled scaffold morphology, mechanical stability, and to prove individual control over the release rate of different drugs within the scaffold.

To do this, amphiphilic copolymers with different block ratio are designed and synthesized by reversible addition-fragmentation chain transfer (RAFT) polymerization 
using $\mathrm{S}, \mathrm{S}^{\prime}$-bis $\left(\alpha, \alpha^{\prime}\right.$-dimethylacetic acid) trithiocarbonate (BDAT) as the effective chain-transfer agent (CTA) (28). The different block ratio is designed to form nanoparticles with different particle size. The theoretical block ratio of hydrophilic to hydrophobic block (PVP:PMMA) was ranged from 1:1.5:1 to 1:6:1 (Table 3.1). The synthesized copolymers were characterized by ${ }^{1} \mathrm{H}-\mathrm{NMR}$ to determine the actual block ratio, as shown in Figure 3.4. As Figure 3.4a shows, the integrated peak ratio at $\sigma: 3.14$ ( $-\underline{\mathrm{C}}_{2}-\mathrm{N}$, h from PVP block) to $\delta: 3.56$ and $3.35\left(-\mathrm{OCH}_{3}\right.$, a from PMMA block, and -C -,e from PVP backbone ) increases as block ratio of PVP:PMMA increase. Using equation (1) and the integrated peak area ratio, the actual block ratio of hydrophilic to hydrophobic block (XPV:X repeat units $\left(\mathrm{X}_{\mathrm{M}}\right)$ of PMMA block and the copolymer molecular weight $\left(\mathrm{M}_{\mathrm{N}}\right)$ were calculated according to the repeat units number of $X_{N}$ and $X_{M}$. The actual block ratio and molecular weight of PHEMA-b-PMMA-b-PHEMA was similarly calculated according to the ${ }^{1} \mathrm{H}-\mathrm{NMR}$ analysis (Fig. 3.4b). The results are shown in Table 3.1. The self-assembled polymeric nanoparticle sizes with different block ratio are tested by DLS and shown in Table 3.2. 


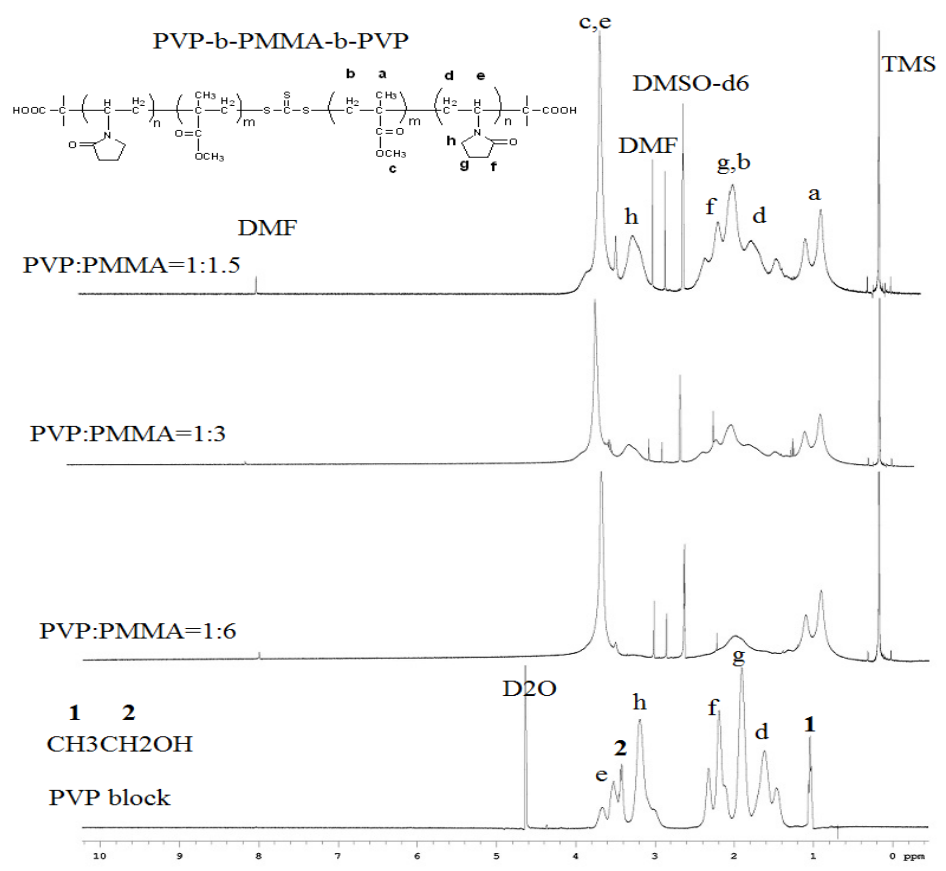

(a)

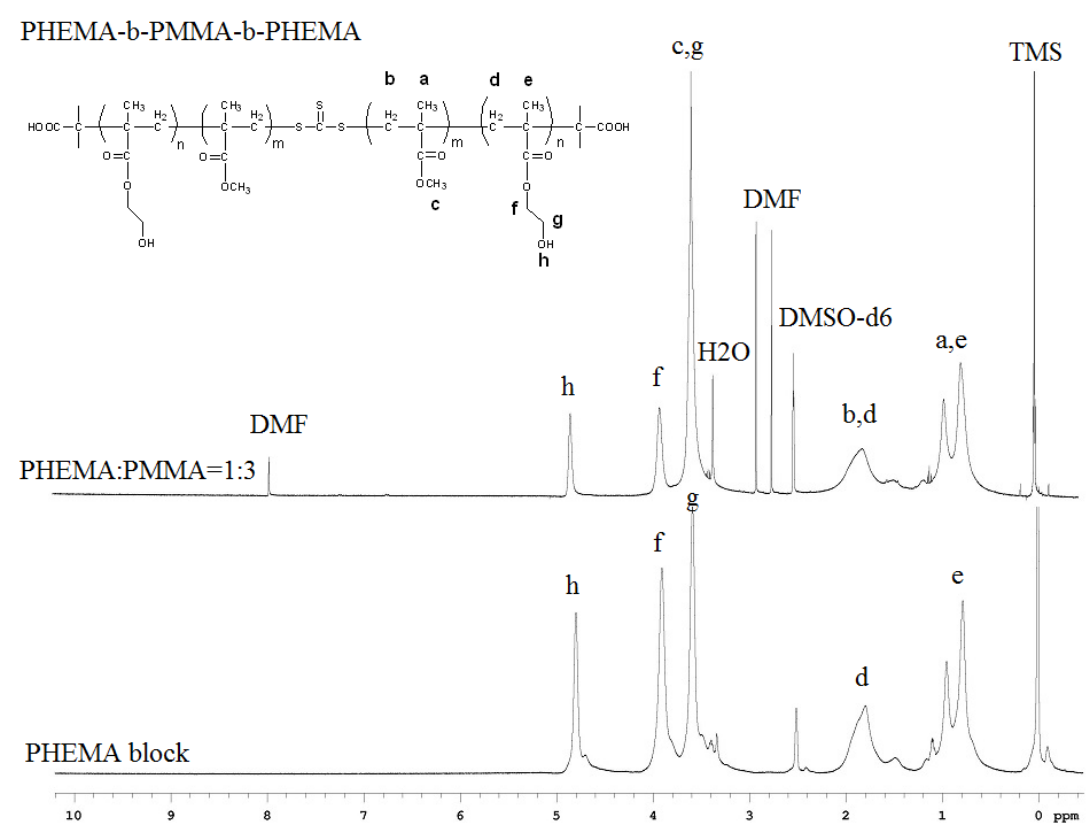

(b)

Figure 3.4. ${ }^{1} \mathrm{H}-\mathrm{NMR}$ spectra of amphiphilic copolymers with different block ratio. (a) PVP and PVP-b-PMMA-b-PVP with block ratio of PVP:PMMA from 1:1.5 to 1:6; (b) PHEMA and PHEMA-b-PMMA-b-PHEMA with block ratio of PHEMA:PMMA=1:3. 


\subsubsection{Coupling reaction of copolymer with peptides}

Each copolymer is then coupled with peptides (P1 and P2) to form P1-copolymer and P2-copolymer conjugates via the coupling reaction between peptide amine terminals and copolymer carboxylic acid terminals, as Scheme 3.1 shows. The peptide-copolymer conjugates were purified by precipitation in cool diethyl ether followed by centrifugation and drying under reduced pressure. The coupling reaction was qualitatively verified by FTIR, as shown in Figure 3.5.

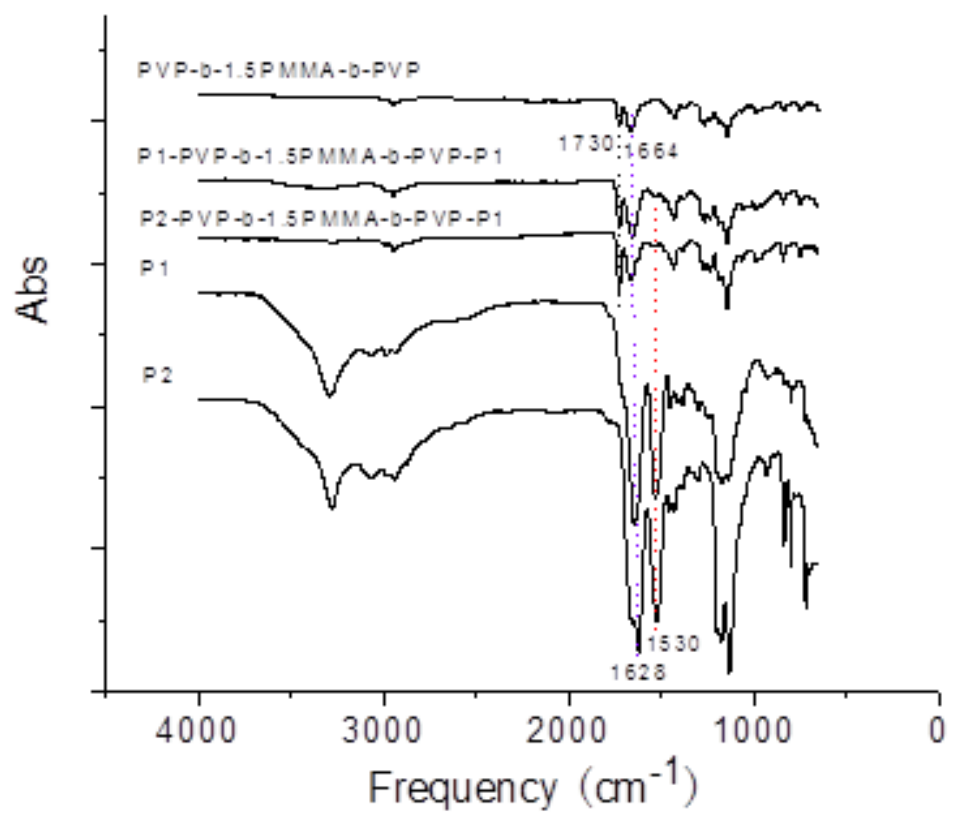

(a) 


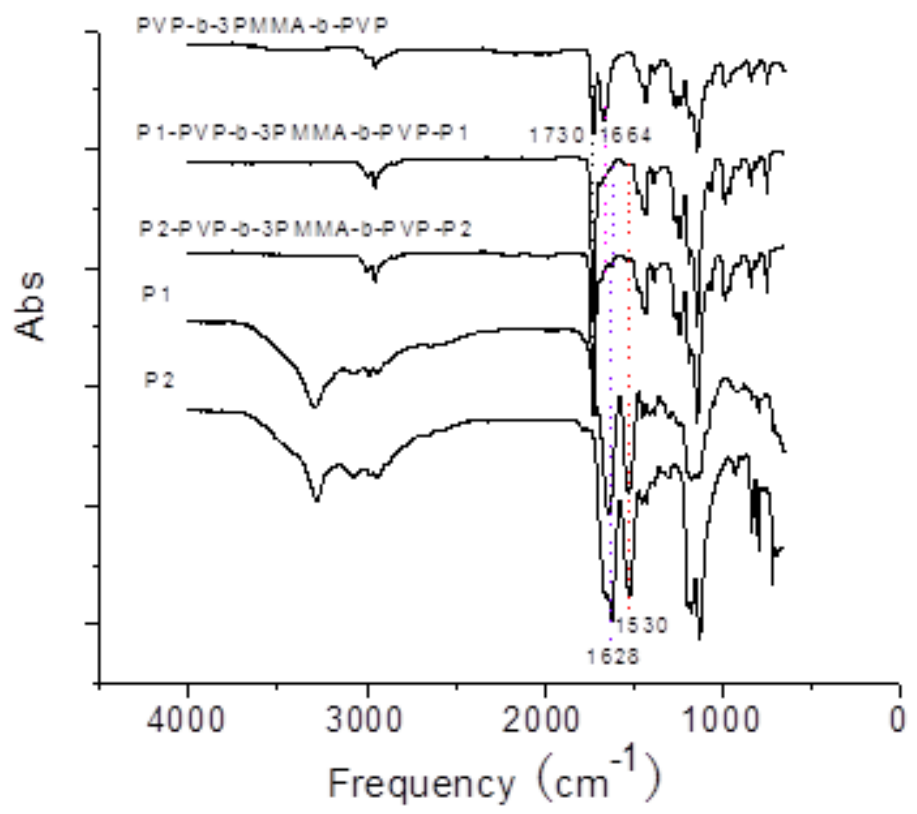

(b)

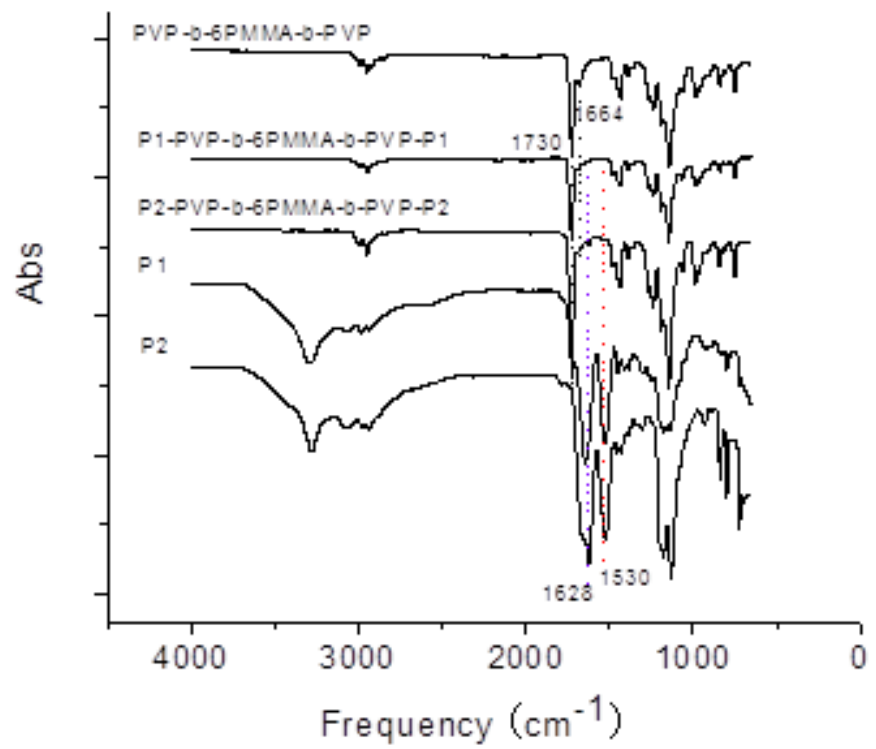

(c) 


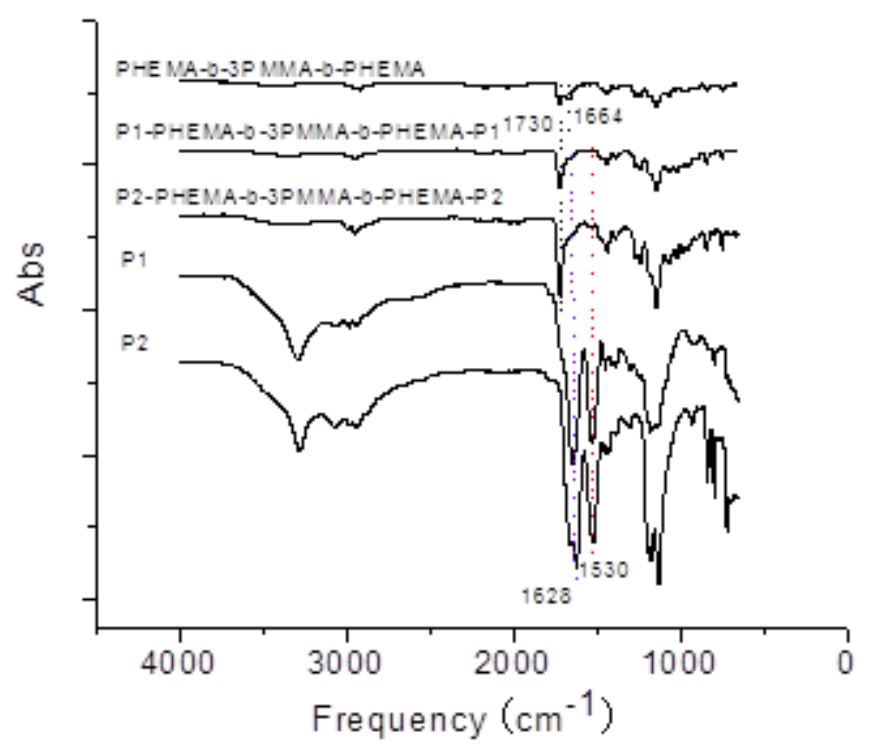

(d)

Figure 3.5. Comparison of FT-IR spectra of peptides (P1 and P2), copolymer and peptide-copolymer conjugates. (a) PVP-b-1.5PMMA-b-PVP coupled with P1 and P2. (b) PVP-b-3PMMA-b-PVP coupled with P1 and P2. (c) PVP-b-6PMMA-b-PVP coupled with P1 and P2. (d) PHEMA-b-3PMMA-b-PHEMA coupled with P1 and P2.

As Figure 3.5 shows, FT-IR spectra of copolymers show two carbonyl absorption peaks at $1730 \mathrm{~cm}^{-1}$ from the PMMA block and $1664 \mathrm{~cm}^{-1}$ from the PVP block. When the hydrophilic block ratio (PVP or PHEMA) is adjusted from 1:6 to 1:1.5, the absorption intensity at $1664 \mathrm{~cm}^{-1}$ also increase.After the copolymer is coupled with peptides ( $\mathrm{P} 1$ and P2), both P1- and P2-copolymer conjugates show weak absorption peaks at approximately $1628 \mathrm{~cm}^{-1}$ and $1530 \mathrm{~cm}^{-1}$, and also feature absorption peaks of the peptide amide bonds from P1 and P2. Also, the original absorption peak at $1664 \mathrm{~cm}^{-1}$ from the hydrophilic block (PVP or PHEMA) is weakened or covered by peptide absorption. These results qualitatively confirm the effective coupling reaction between copolymer with peptides to form peptide-copolymer conjugates. 


\subsubsection{Self-assembly study of peptide-functionalized nanoparticles}

The purpose of this work is to fabricate self-assembled 'nanoparticle fibers' that can subsequently self-assemble to produce 3D scaffolds as directed by $\beta$-sheet peptide assembly with multi-drug loading abilities (Fig. 3.1). In previous work we already showed that peptide-functionalizedmicroparticles with particle size of $\sim 1 \mu \mathrm{m}$ could effectively assemble to form 1D nanoparticle fibers and eventually lead to 3D scaffolds after condensation (Fig. 3.6).

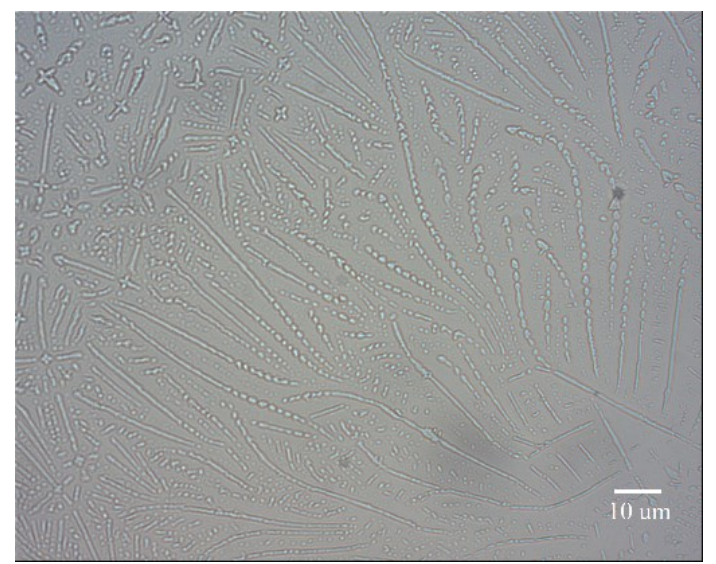

(a)

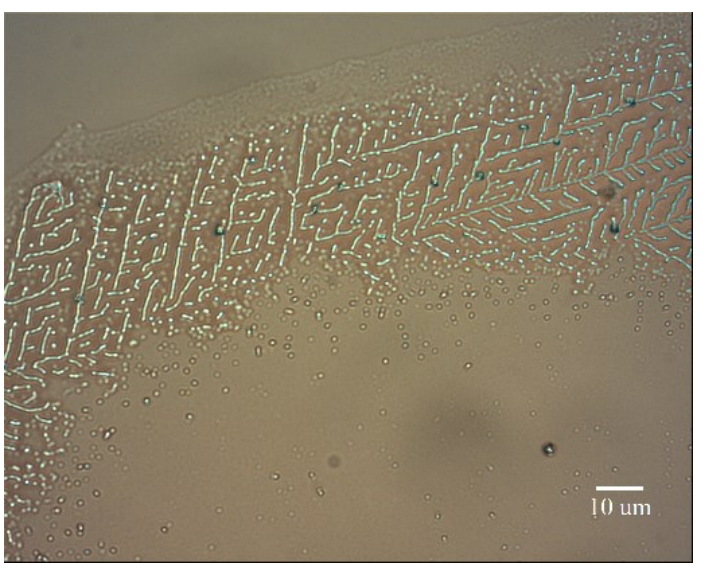

(c)

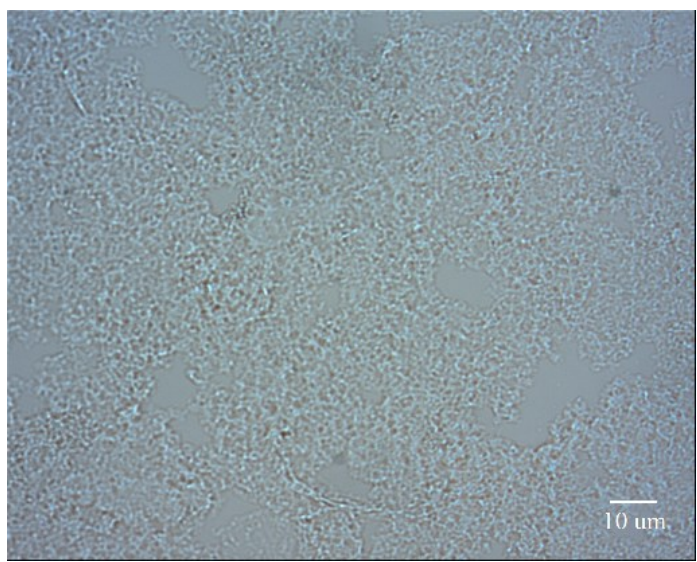

(b)

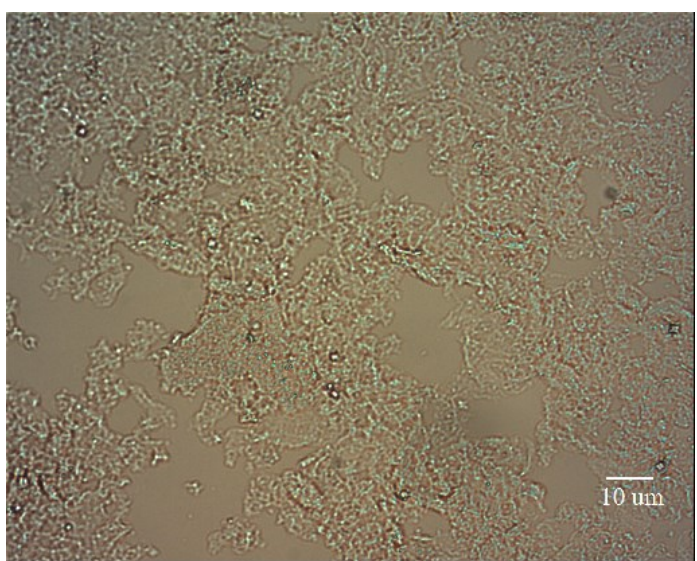

(d)

Figure 3.6. Optical microscopy images of self-assembled 1D nanoparticle fibers and 3D nanoparticle scaffolds on glass slide surfaces. Microparticle fibers and scaffolds from 
peptide-functionalized PVP-b-12PMMA-b-PVPconjugates are shown in(a) and (b). Microparticle fibers and scaffolds from peptide-functionalized PHEMA-b-12PMMA-b-PHEMA conjugates are shown in(c) and (d).Both 1D nanoparticle fibers wereprepared by gently shaking self-assembled nanoparticle suspension (10 $\mathrm{h}$ assembling time) and then transferring via $31 \mathrm{G}$ syringe onto glass slide surface with slowly moving syringe needle down along slide surface. Both 3D scaffolds were prepared without shaking self-assembled nanoparticle suspension.

The effect of particle size on the self-assembly behavior, the assembled scaffold morphology and its mechanical stability were also studied. The self-assembled nanoparticles from various peptide-copolymer conjugates with different block ratios (Table 3.1) were tested by dynamic light scattering (DLS) with particle size ranging from $178 \pm 72 \mathrm{~nm}$ to $580 \pm 264 \mathrm{~nm}$ as the length of the hydrophobic block (PMMA block) was increased (Table 3.2). The self-assembly process between P1-NP-P1 and P2-NP-P2 is illustrated in Figure 3.7.
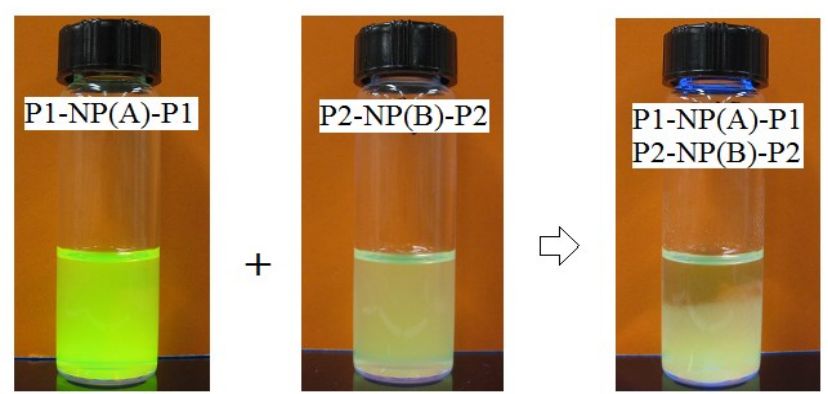

Figure 3.7. Self-assembly process between $\mathrm{P} 1-\mathrm{NP}_{\mathrm{A}}-\mathrm{P} 1$ and $\mathrm{P} 2-\mathrm{NP}_{\mathrm{B}}-\mathrm{P} 2$ with $\mathrm{DBF}$ loading as indicator.

The peptide-functionalized nanoparticle pair $\left(\mathrm{P} 1-\mathrm{NP}_{\mathrm{A}}-\mathrm{P} 1\right.$ and $\left.\mathrm{P} 2-\mathrm{NP}_{\mathrm{B}}-\mathrm{P} 2\right)$ were loaded with DBF as an indicator to verifythe self-assembly. As Figure 3.7 shows, initially stable $\mathrm{P} 1-\mathrm{NP}_{\mathrm{A}}-\mathrm{P} 1$ and $\mathrm{P} 2-\mathrm{NP}_{\mathrm{B}}-\mathrm{P} 2$ suspensions are coupled together with magnetic stirring. The self-assembly between these two nanoparticles begins once 
magnetic stirring is stopped. It is directed by the ionic complementary assembly between $\mathrm{P} 1$ and P2, and forms a 'sponge-like' bottom layer in the vial. Our study shows that the self-assembly proceeds more quickly for smaller nanoparticles $(3 \mathrm{~h}$ for P1-NP1-P1 and P2-NP1-P2) than for bigger particles (10 h for P1-NP3-P1 and P2-NP3-P2) (Table 3.2).

The self-assembled 2D nanoparticle scaffold membrane was prepared by transferring $\sim 100 \mu \mathrm{L}$ of the 'sponge-like' layer onto the silicon wafer surface and air dried, which was then coated with $5 \mathrm{~nm} \mathrm{Pt} / \mathrm{Pd}$ layer. FESEM micrographs of the scaffold membrane with different particle size are shown in Figure 3.8.

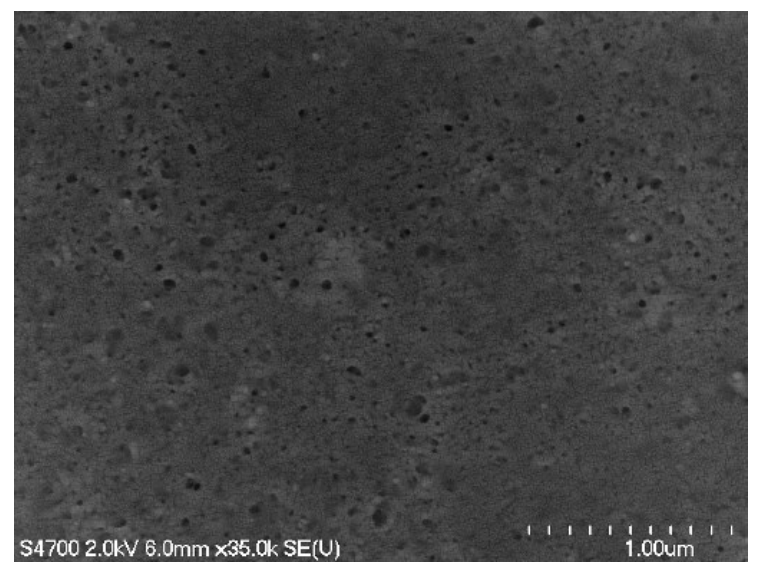

(a)

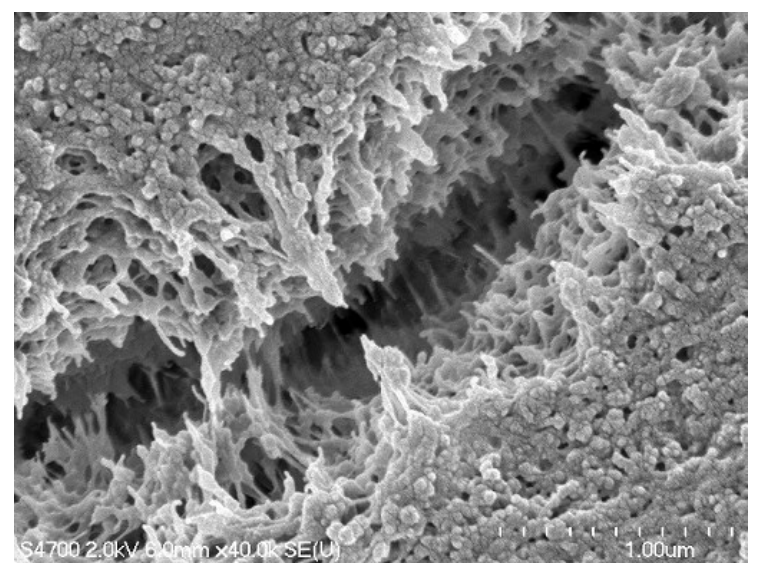

(c)

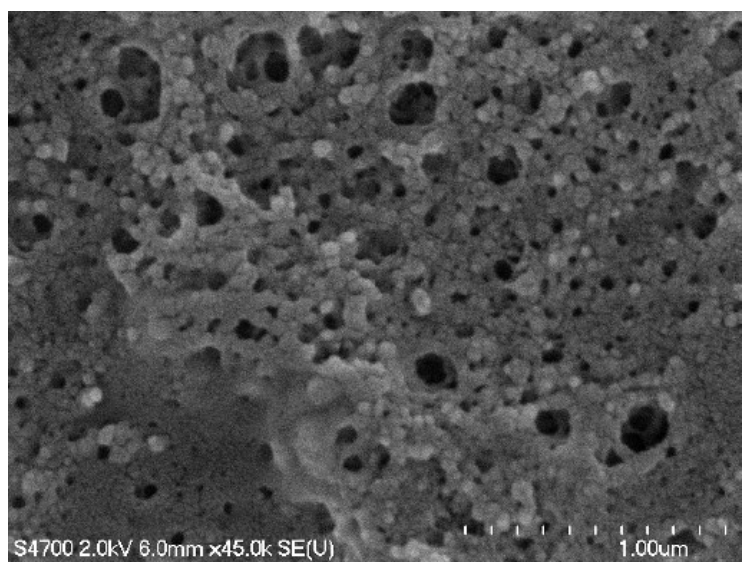

(b)

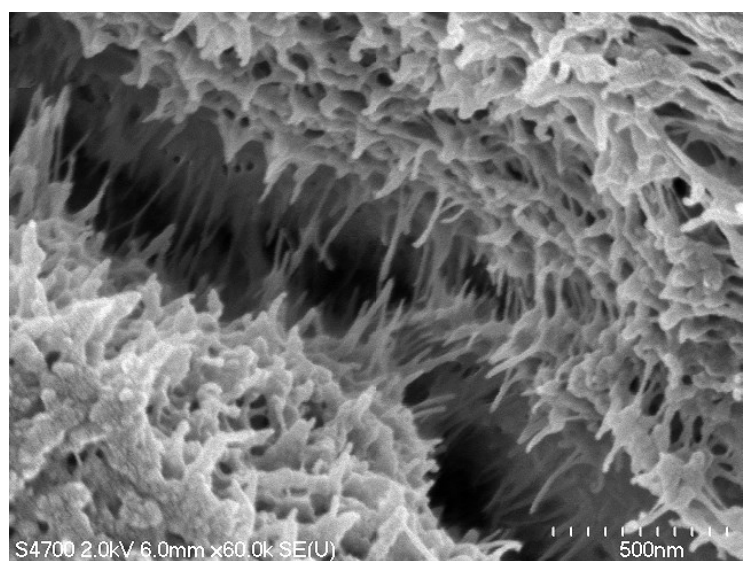

(d)

Figure 3.8. FESEM micrographics of self-assembled nanoparticle membrane on silicon wafer surface after being air dried. (a) Scaffold membrane assembled from P1-NP1-P1 
and P2-NP1-P2; (b) Scaffold membrane assembled from P1-NP3-P1 and P2-NP3-P2; (c) Cleaved scaffold interface morphology from P1-NP3-P1 and P2-NP3-P2; (d) Scaffold loaded with 0.2 wt.\% of model drug (DBF) within nanoparticles.

Figure 3.8 shows the morphologies of the assembled 2D scaffold membrane on silicon wafer surfaceand air dried under room temperature. The smaller nanoparticles (P1-NP1-P1 and P2-NP1-P2, DLS size: 178 $\pm 72 \mathrm{~nm}$ ) self-assembled to form more compact membrane with smooth surface and smaller pores (Fig. 3.8a), while bigger nanoparticles (P1-NP3-P1 and P2-NP3-P2, DLS size: 580 $264 \mathrm{~nm}$ ) assembled to formmembrane with rough surface and bigger pores (Fig. 3.8b). However, the micrographics from the cleaved interface clearly show 3D fibrous structure inside of the scaffold membrane with or without loading of model drug (DBF) (Fig. 3.8c, d). The FESEM micrographics also show that the dried nanoparticle size is about 20-50 nm for smaller nanoparticles and 50-100 $\mathrm{nm}$ for bigger nanoparticles, which is much smaller than the size tested by DLS, mainly due to hydrodynamic size of wet nanoparticles and slight aggregation in aqueous solution.

On comparing themorphologies ofscaffold membranesassembledfrom smaller and larger nanoparticles (Fig. 3.8a and b) it is not difficult to understand that smaller nanoparticles undergo faster self-assembly than the larger ones (Table 3.2) because smaller nanoparticlescan move more easily to be directed by electrostatic interactions between $\mathrm{P} 1$ and $\mathrm{P} 2$, and the nanoparticles themselves have stronger inter-particle interaction force. Therefore, the smaller nanoparticles self-assemble to form more compact scaffolds with smaller pores, but are more mechanically stable than those from larger nanoparticles. Therefore there is a balance between nanoparticle size and mechanical stability and pore size for such assembled nanoparticle scaffolds when they are to be used for tissue engineering.

\subsubsection{Self-assembly study of peptide-functionalized nanoparticle with additional}




\section{peptides}

In order to further increase the mechanical stability and combine the advantage of self-assembling peptide scaffolds, peptide-functionalized nanoparticles (P1-NP3-P1 and P2-NP3-P2, total concentration $1.3 \mathrm{mg} / \mathrm{mL}$ ) are co-assembled with additional peptides (P1 and $\mathrm{P} 2)$ with total peptide concentration $(\mathrm{P} 1: \mathrm{P} 2=1: 1 \mathrm{wt} . / \mathrm{wt}$.$) ranged from 5.5 \mathrm{mg} / \mathrm{mL}$ to $22 \mathrm{mg} / \mathrm{mL}$. The co-assembled composite gel status is shown in Figure 3.9. From left to right, as the peptide concentration increases, the stability of the co-assembled composite gels are correspondingly increased by forming gels, but self-assembled nanoparticle suspension control (Fig. 3.9a) shows at flow status with 'sponge-like appearance'. The stability of composite gels is compared by sonicating 30 seconds, only nanoparticles co-assembled with $22 \mathrm{mg} / \mathrm{mL}$ of peptides remained stable gel status (Fig. 3.9d), while the composite gels with low peptide concentration was broken into viscous solution status (Fig. 3.9b,c).

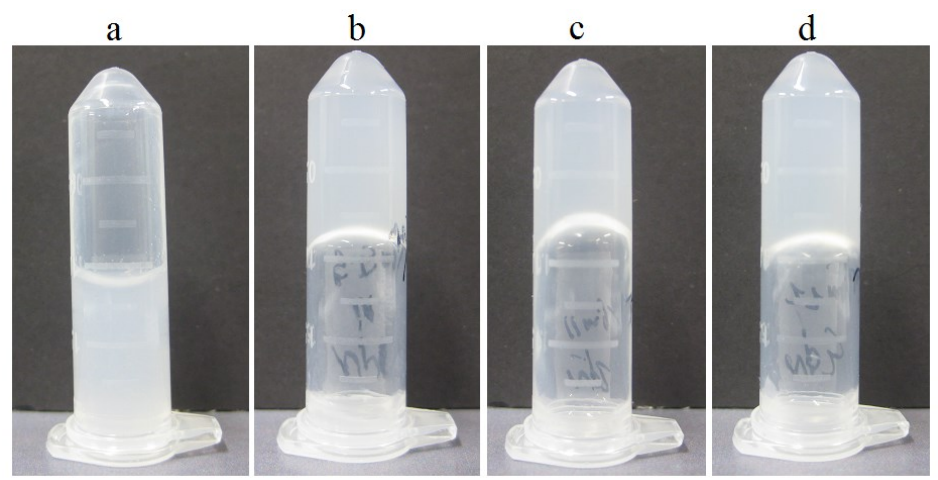

Figure 3.9. Peptide-functionalized nanoparticles (P1-NP3-P1 and P2-NP3-P2) co-assembled with additional peptides (P1+P2, 1:1 wt./wt.) to form composite peptide gels. (a) Self-assembled nanoparticle control without peptides; (b) With total peptide concentration at $5.5 \mathrm{mg} / \mathrm{mL}$; (c) With total peptide concentration at $11 \mathrm{mg} / \mathrm{mL}$; (d) With total peptide concentration at $22 \mathrm{mg} / \mathrm{mL}$.

The self-assembled morphologies of P1-NP3-P1 and P2-NP3-P2 with additional 
peptides are further characterized by FESEM microscopy, as shown in Figure 3.10. FESEM micrographics of Figure 3.10 a,b,c are prepared by dropping composite gels on silicon wafer surface and air dried under room temperature with washing away un-bonded peptides by rinsing with deionized water $(3 \times 100 \mu \mathrm{L})$. While Figure 3.10 d,e,f are morphologies of composite gel co-assembled with $22 \mathrm{mg} / \mathrm{mL}$ of peptides without washing and dried at room temperature under reduced pressure in a vacuum oven.

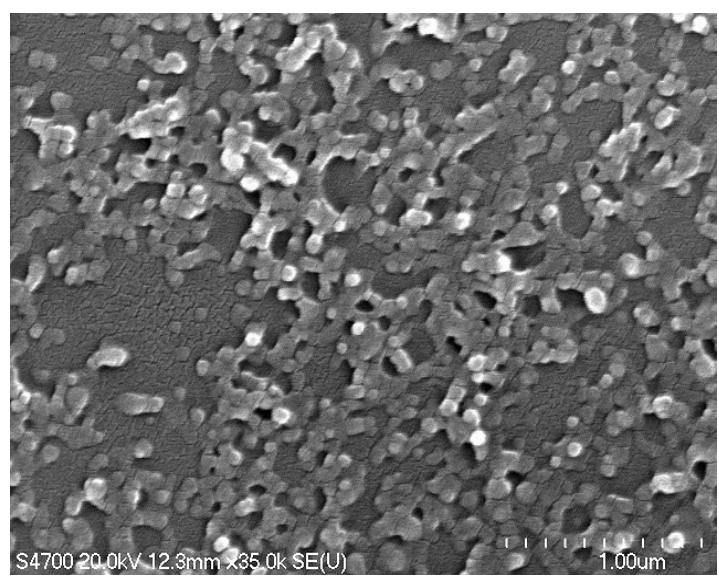

(a)

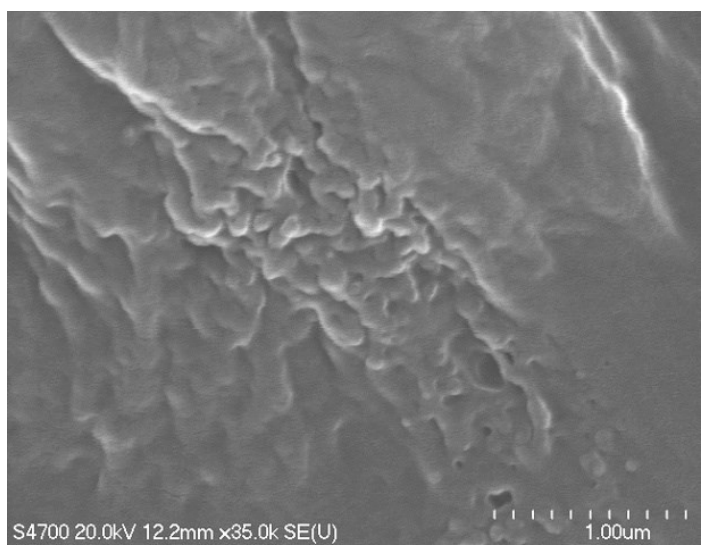

(b)

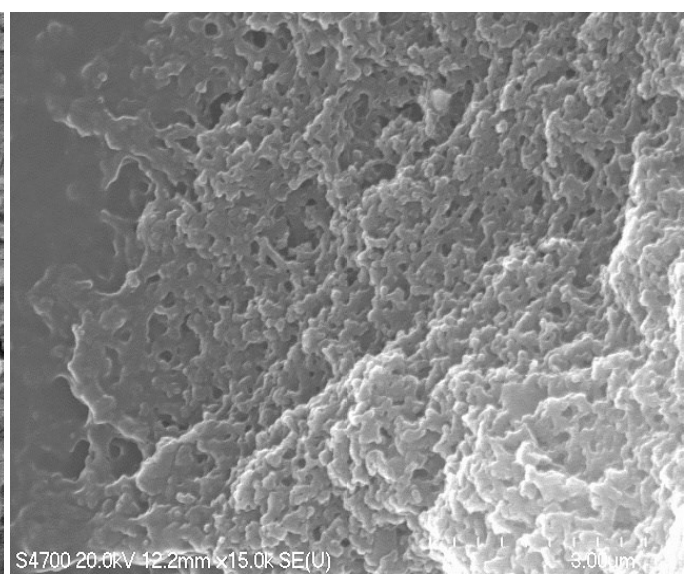

(b)

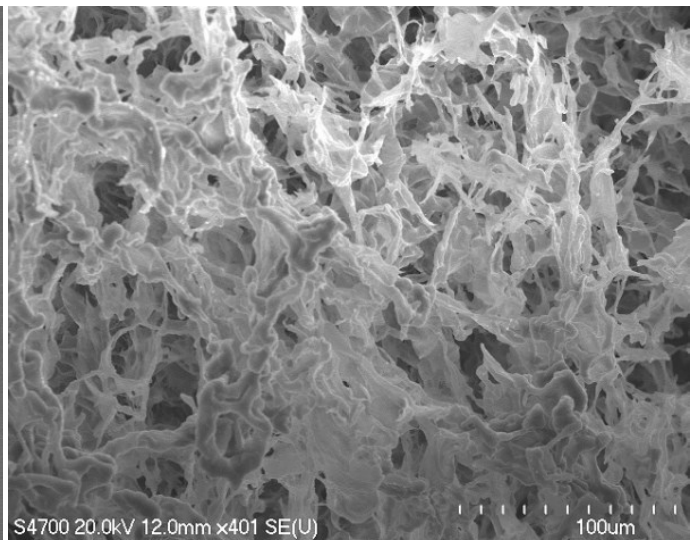

(d) 


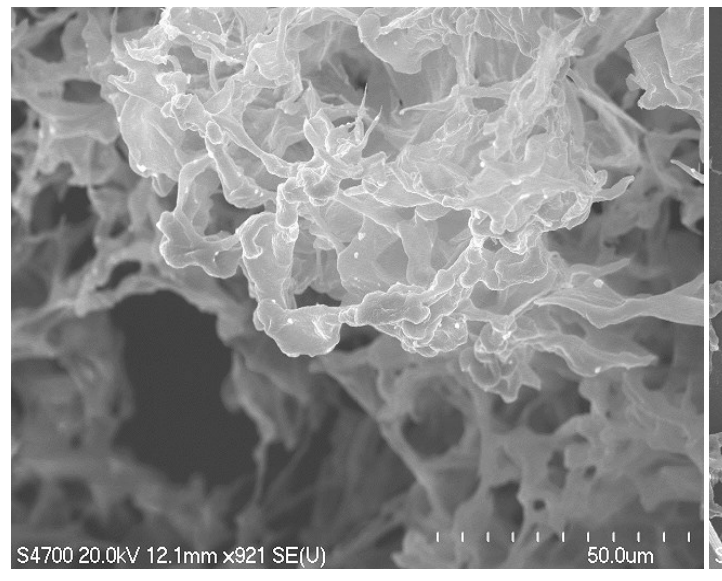

(e)

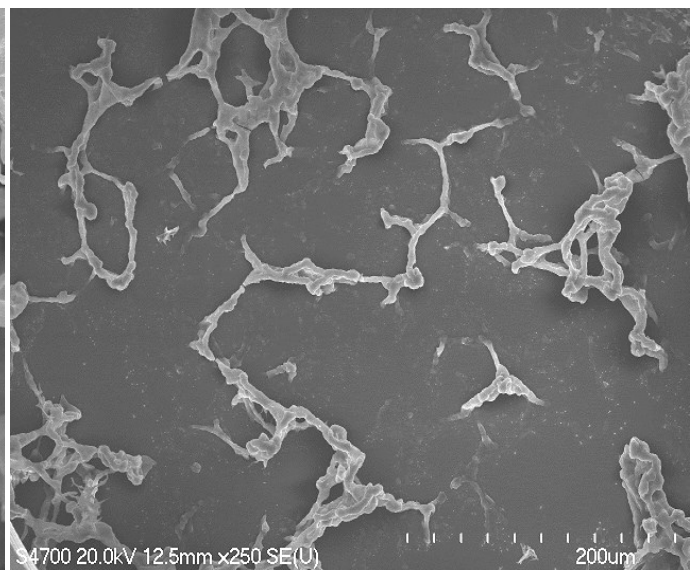

(f)

Figure 3.10. FESEM micrographs of the co-assembled composite peptide gels prepared by assembling of peptide-functionalized nanoparticles (P1-NP3-P1 and P2-NP3-P2) with additional ionic complementary peptides (P1 and P2) at different total peptide concentration and then transferring onto silicon wafer surface.(a) Co-assembled gel with peptide concentration at $5.5 \mathrm{mg} / \mathrm{mL}$; (b) Co-assembled gel with peptide concentration at $11 \mathrm{mg} / \mathrm{mL}$; (c) Co-assembled gel with peptide concentration at $22 \mathrm{mg} / \mathrm{mL}$.(a), (b) and (c) are gel membranes on silicon wafer surface after air drying and washing with deionized water. (d), (e) and (f) are representative morphologies of co-assembled gel with peptide concentration at $22 \mathrm{mg} / \mathrm{mL}$ without washing and dried at room temperature under reduced pressure.

Comparing the morphologies of self-assembled membrane on silicon wafer surface by air drying and washing with deionized water, the self-assembled nanoparticles without additional peptides formed compact porous 3D nanoparticle scaffolds with fiber structure (Fig. 3.8 b,c).However, the co-assembled composite gels with additional peptides at concentration from $5.5 \mathrm{mg} / \mathrm{mL}$ to $22 \mathrm{mg} / \mathrm{mL}$ appear that assembled nanoparticles are embedded into peptide layers without apparent 3D network structure after washed with 
deionized water (Fig. 3.10 a,b,c), even though the membrane is stably stick onto silicon wafer surface. Again, the conversion to amorphous aggregation morphology is caused by water washing due to high water solubility of the designer peptides ( $\mathrm{P} 1$ and $\mathrm{P} 2)$. This is evidenced by comparing the morphology change of the co-assembled composite gel with $22 \mathrm{mg} / \mathrm{mL}$ of peptides before and after washing with deionized water, and air dried or dried under reduced pressure (Fig. 3.10 c compared with d,e,f). It is shown that the co-assembled composite gel membrane is amorphous aggregation without any 3D network structure (Fig. 3.10c) if washed deionized water and air dried, but Figure 3.10 d,e,f clearly show the 3D fibrous structurewith somewhat film structure if without washing and dried under reduced pressure.

Interestingly, there are several new features from this co-assembled composite gel scaffoldwith total peptide concentration at $22 \mathrm{mg} / \mathrm{mL}$ comparing with other reported ionic complementary peptide nanofiber scaffold. At first, it shows fiber structure withmicroscale diameter, much larger than conventionalself-assembling peptide nanofiber (29), even though our co-assembled fiber diameter is not in uniform size. Secondly, the single fiber length is ranging from tens to hundreds micrometer, also much longer than traditional peptide nanofibers with length from hundreds nanometer to several micrometers (29). Thirdly, the 3D scaffolddominant structure is from self-assembling peptide scaffolds but with microscale porous structure, no apparent nanoparticle scaffolds is visible from both FESEM images. This is considered that peptide-functionalized nanoparticles are effectively co-assembled with host peptides and uniformly distributed in peptide scaffolding system. These new features probably will expand the peptide scaffold applications where requires larger porosity with longer diameter, ease of multiple drug loading capability and controlled release rate of each drug. 


\subsubsection{Controlled release test of multi-drug models}

The purpose of this project is to design self-assembled polymeric nanoparticle scaffolds with the ability to load different types of drugs allowing for controlled release for each drug. In prior work insulin was used as macromolecular model drug and loaded into nanoparticle scaffolds ( 0.15 wt. $\%$ loading quantity) without destroying the self-assembled structure. This system showed sustainable release over 3 weeks (26). To further study multi-drug loading abilities and the simultaneous sustainable release of each drug, several compounds with different properties are used as model drugs and loaded in nanoparticle scaffolds, and the controlled release was tested. The compounds were DBF as a hydrophobic small molecule model, nitrofurazone as a less hydrophobic small molecule model and amoxicillin as hydrophilic small molecule model drug.

The theoretic and actual loading levels of the model drugs in each type of nanoparticles are summarized in Table 3.3 and Table 3.4. The cumulative release of each model drug from the self-assembled nanoparticle scaffolds is shown in Figure 3.11.

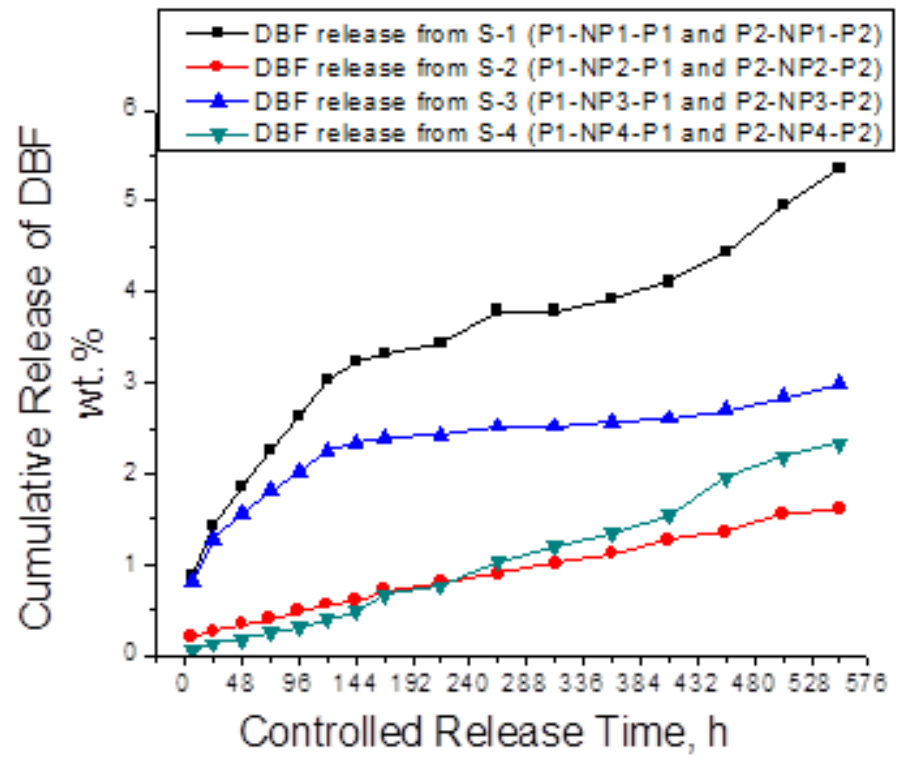

(a) 


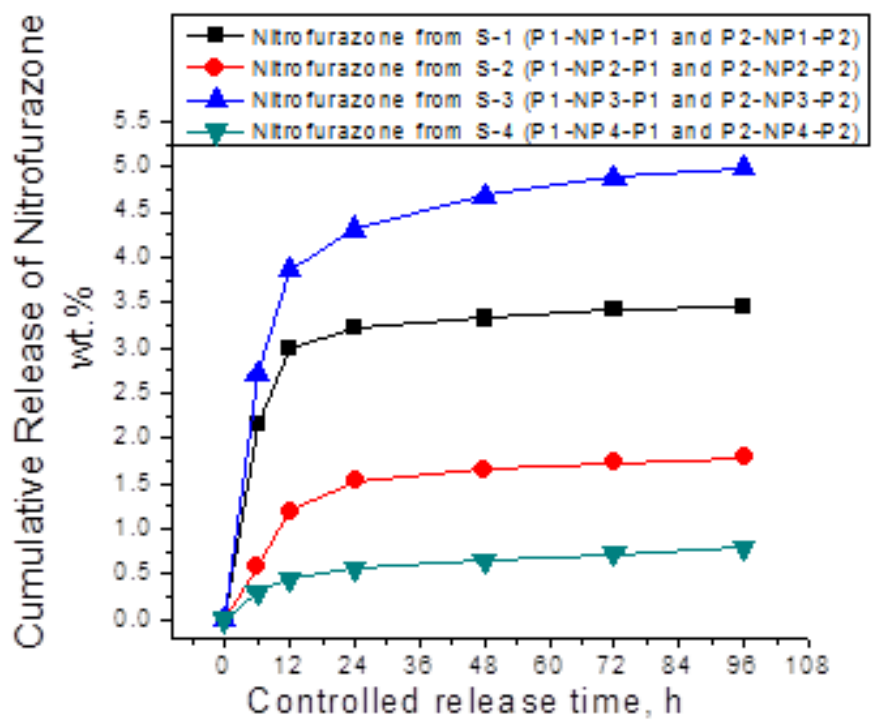

(b)

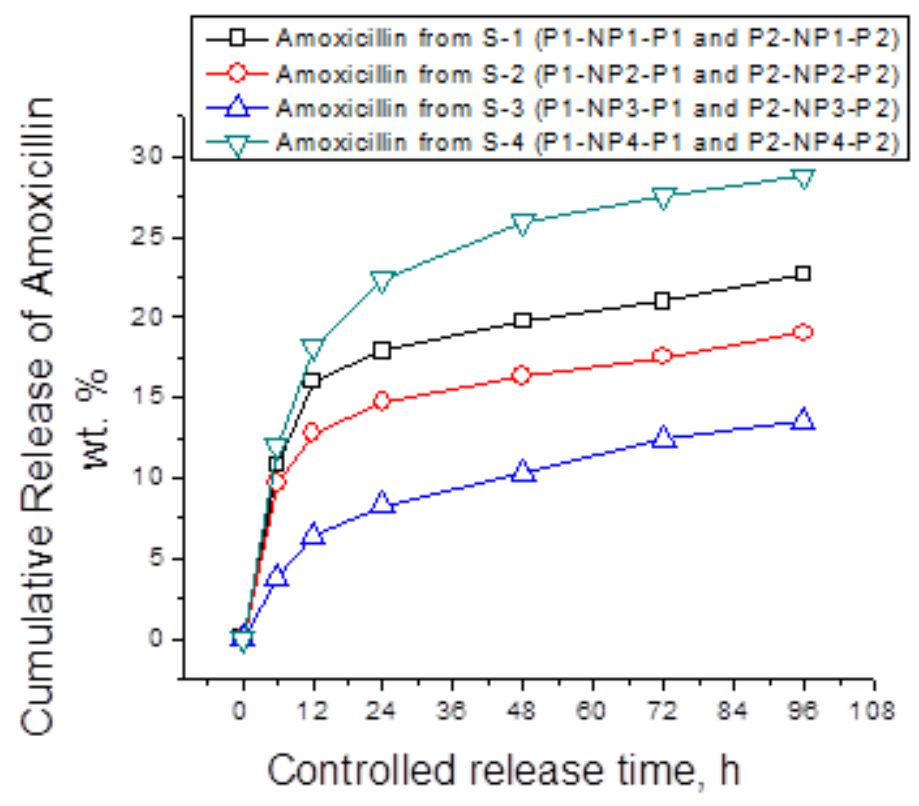

(c)

Figure 3.11. Cumulative release (wt.\%) of model drugs from self-assembled nanoparticle scaffolds with different particle size and composition.(a) Cumulative release of DBF from various assembled scaffolds; (b) Simultaneous cumulative release of nitrofurazone from 
self-assembled scaffolds; (c) Simultaneous cumulative release of amoxicillin from self-assembled scaffolds.

DBF is first used as hydrophobic small molecule model drug and loaded in both P1-NP-P1 and P2-NP-P2 to perform controlled release test over 3 weeks from self-assembled scaffolds to determine long release period. The theoretical loading quantity is set up to $0.2 \mathrm{wt} . \%$ and high loading efficiency is achieved between 85 to $95 \%$ for these hydrophobic small molecules. The releasing profile in Figure 3.11a shows only 1.5 to 5.5 wt.\% of DBF released from scaffold over 3 weeks, and smaller nanoparticles generally released more quickly than bigger ones, except S-3 showing faster than from S-2 (Table 3.3, S-3 has bigger particle size than S-2).

Simultaneous loading of multiple drugs and governing release rate of each drug from same scaffold is one of the main objectives of this project. Here we use nitrofurazone (less hydrophobic small molecule) and amoxicillin (slight water soluble hydrophilic small molecule) as model drugs to load in P1-NP-P1 and P2-NP-P2 separately, and then couple these two peptide-functionalized nanoparticles to self-assemble into scaffold to perform simultaneous controlled release test of both drugs from same scaffold. For these two model drugs, the theoretical loading quantity is increased to $5.0 \mathrm{wt} . \%$ with actual loading efficiency ranging from 58 to $94 \%$ for nitrofurazone and from 24 to $32 \%$ for amoxicillin. Again, the high loading efficiency for less hydrophobic small molecules (nitrofurazone) is still achieved though the loading quantity is increased to $5.0 \mathrm{wt} . \%$. While loading efficiency for hydrophilic small molecule (amoxicillin) is relatively reduced due to its water solubility.

Figure 3.11 b,c show the simultaneous releasing profiles from the self-assembled nanoparticle scaffolds. Two main results can be read from their releasing profiles: (1) Hydrophilic molecules (amoxicillin) release more quickly than hydrophobic molecules (nitrofurazone); (2) The general trends are that smaller nanoparticles release drugs more 
quickly than bigger nanoparticles $(\mathrm{S}-1>\mathrm{S}-2>\mathrm{S}-3)$, where hydrophobic core (PMMA) is increased for these nanoparticle scaffolds, except nitrofurazone released from S-3 showed inverse trend (Fig. 3.11 b).

While comparing release behavior of nitrofurazone and amoxicillin from S-2 and S-4 (self-assembled from peptide-functionalized PVP-b-3PMMA-b-PVP and PHEMA-b-3PMMA-b-PHEMA), the nanoparticle composition of hydrophilic shell is changed, but the particle size is very close, the releasing behavior shows significant difference. It appears that hydrophilic shell of PHEMA enhanced amoxicillin release rate, but reduced nitrofurazone release. This is most likely due to hydrogen bonding interaction with drugs from hydroxyl groups of PHEMA shell, while PVP shell does not possess such groups. This means the shell property is another factor to govern release rate of drugs with different hydrophilicity, but not only be controlled by hydrophobic core domain and particle size.

Even though the detailed release rate of drugs from core-shell nanoparticles is complexly influenced by several factors, such as drug properties, actual loading quantity, core-shell ratio, porous structure and shell properties, the basic principle is that hydrophobic molecules (i.e. DBF and nitrofurazone) prefer to be encapsulated into hydrophobic core (i.e. PMMA) of the self-assembled core-shell polymeric nanoparticles, while hydrophilic molecules (i.e. amoxicillin) are more likely to be captured in hydrophilic shell (i.e. PVP or PHEMA). So the general trends are that the bigger hydrophobic core domain more easily encapsulates with hydrophobic molecules, while the hydrophilic molecules are more likely to be captured in more hydrophilic shell domain. The releasing rate of hydrophobic drugs (i.e. DBF or nitrofurazone) is mainly governed by diffusion through hydrophobic core to hydrophilic shell and then entering into aqueous media, so that they release more quickly from smaller nanoparticles than bigger ones. While the hydrophilic drugs (i.e. amoxicillin) are mostly captured in 
hydrophilic shell and thus have shorter diffusion route to aqueous media, showing higher releasing rate, but release rate can be affected by both diffusion speed and molecular interaction force with shell functional groups.

\subsubsection{Cytotoxicity test with NIH3T3 cell lines}

The cytotoxicity test of the self-assembled scaffolds with different particle size and composition was conducted by incubating Fibroblast cells (NIH3T3 cell lines) with self-assembled nanoparticle scaffold membrane ( $\sim 500 \mu \mathrm{g}$ for each scaffold membrane) in each glass culture dish with cell media in a humidified incubator with $5 \% \mathrm{CO}_{2}$ at $37{ }^{\circ} \mathrm{C}$. The cell growth behavior was monitored by optical microscopy observation every $24 \mathrm{~h}$ with total testing period for one week. At $72 \mathrm{~h}$, the optical microscopy images of the cell proliferation status in each culture dish were recorded and the results are shown in Figure

\subsection{2.}

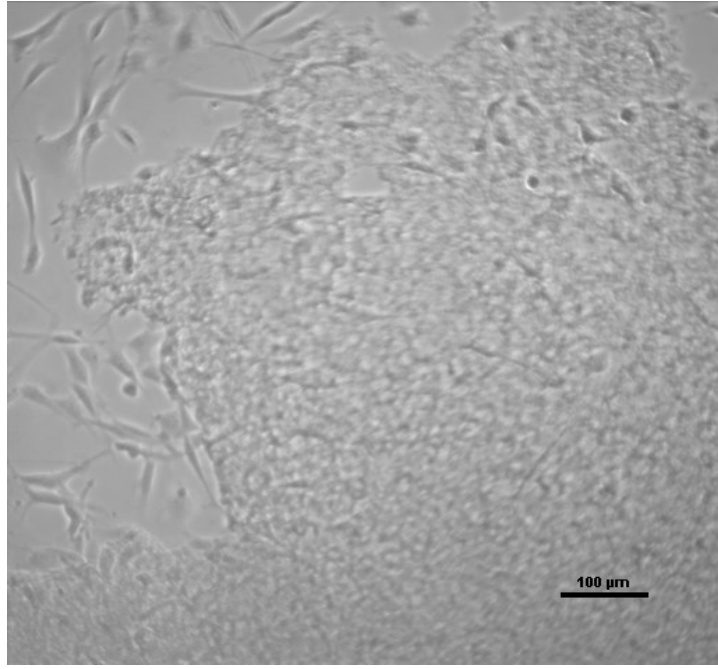

(a)

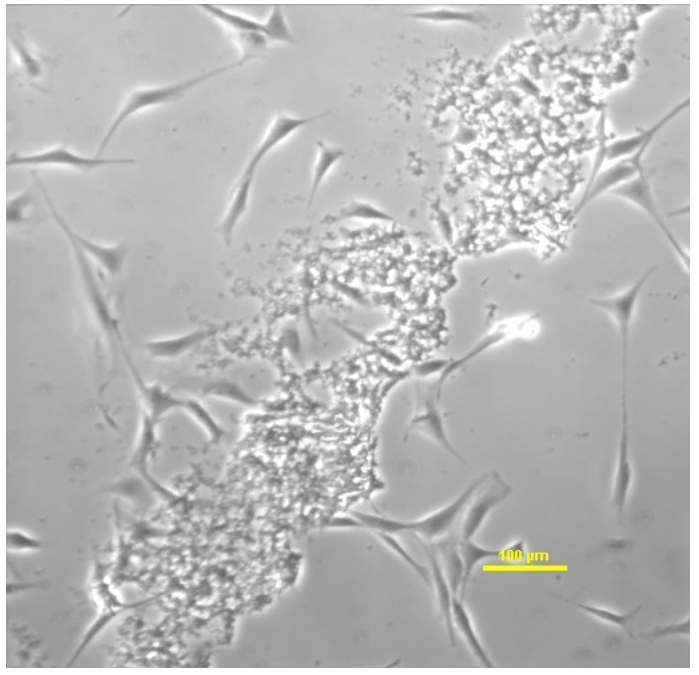

(b) 


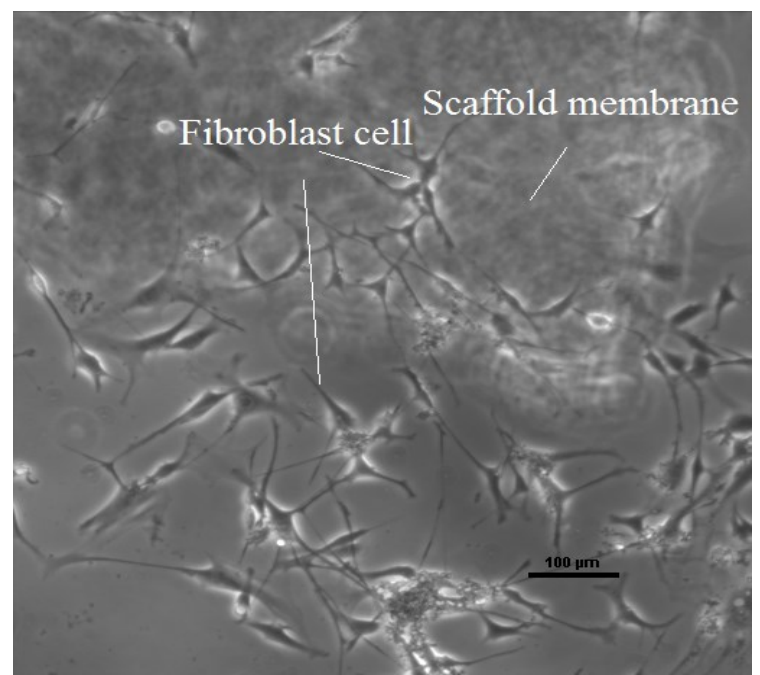

(c)

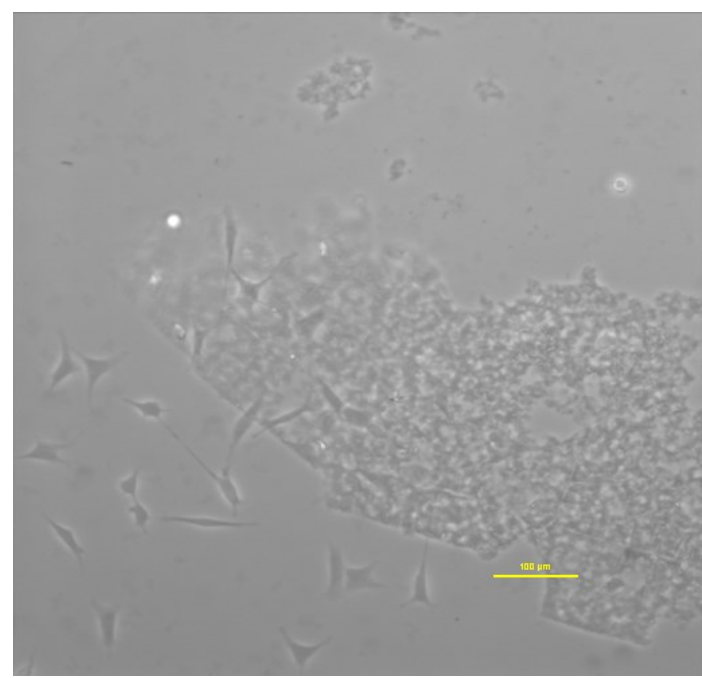

(d)

Figure 3.12. Cytotoxicity tests of an assembled scaffold membrane with Fibroblast cells in a glass culture dish. The micrographs were recorded after incubating for $72 \mathrm{~h}$ in a humidified incubator with $5 \% \mathrm{CO}_{2}$ at $37^{\circ} \mathrm{C}$. The nanoparticle scaffold samples in each culture dish are: (a) S-1 from P1-NP1-P1and P2-NP1-P2; (b) S-2 from P1-NP2-P1and P2-NP2-P2; (c) S-3 from P1-NP3-P1and P2-NP3-P2; (d) S-4 from P1-NP4-P1 and P2-NP4-P2 (see Table 3.3). Scale bar $100 \mu \mathrm{m}$.

Figure 3.12 shows that the nanoparticle scaffold membranes, prepared with different particle sizesand copolymer compositions (S-1 to S-4, Table 3.3) showedno toxicity nor did they inhibit fibroblast cell growth at concentrations of $\sim 500 \mu \mathrm{g}$ scaffold $/ 5 \mathrm{~mL}$ cell media. All theself-assembled scaffold membrane remained stable in the cell media throughout the incubation period, except S-3 (assembled from P1-NP3-P1 and P2-NP3-P2, Fig. 3.12 c), which broke down into small pieces after incubating $72 \mathrm{~h}$ in cell media. However, optical microscopy images also showed that the fibroblast cells did not effectively adhere to this membrane surface for migration and proliferation.

In order to improve cell adhesion to membrane surface, we tried to physically incorporate cell adhesion peptides (RGDS) into nanoparticles and then self-assemble into 
2D membrane for cell tests.Here we loaded peptides (RGDS, 1 wt.\%) in nanoparticles of both P1-NP2-P1 and P2-NP2-P2, and P1-NP4-P1 and P2-NP4-P2 (Table 3.3), to self-assemble into RGDS-loaded S-2 and S-4. The peptide-loaded scaffold membrane was similarly prepared and incubated with fibroblast cells in a humidified incubator for 1 week with optical microscopy observation every $24 \mathrm{~h}$. Figure 3.13 shows optical micrographics taken after incubating 108 h.Again, both scaffold membrane did not show toxicity or inhibition to cell growth with stable structure. But unfortunately, our study shows that physical incorporation of cell adhesion peptides in nanoparticle scaffold did not apparently improve cell adhesion efficiency to membrane surface.

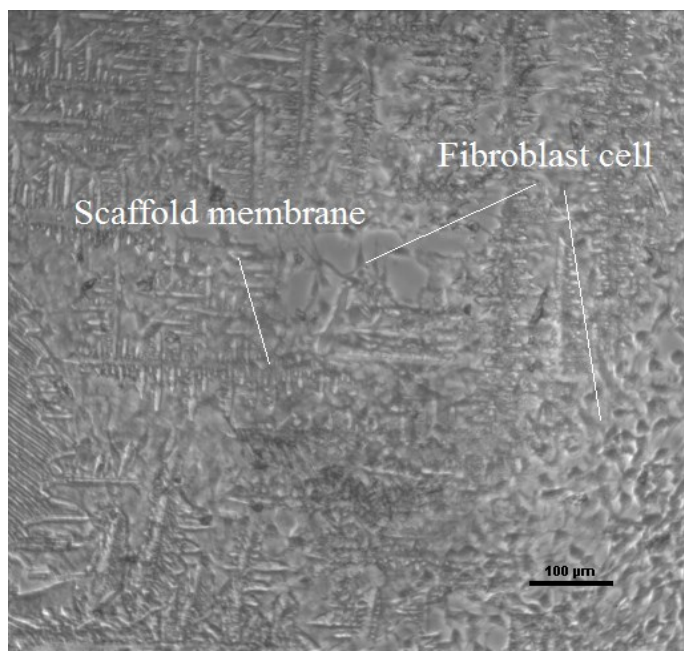

(a)

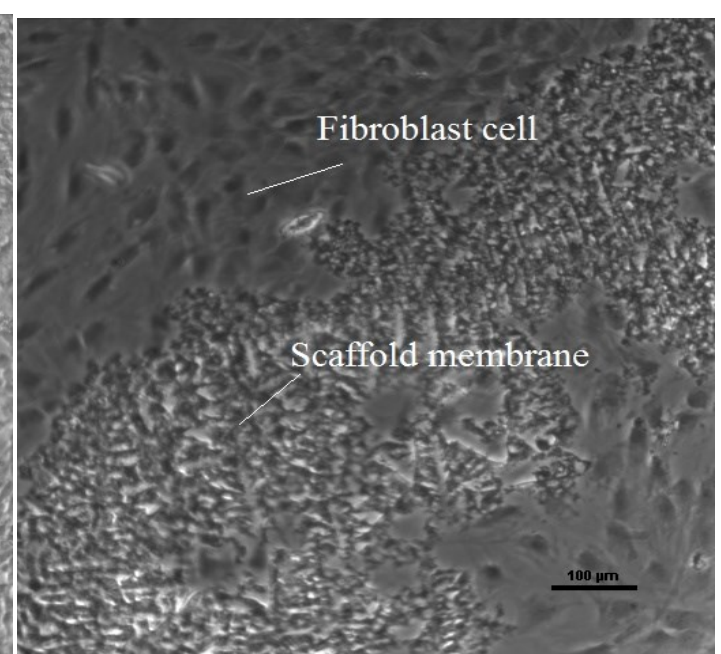

(b)

Figure 3.13. Fibroblast cell proliferation and migration on self-assembled nanoparticle membrane surface after the nanoparticle was physically incorporated with cell adhesion peptides (RGDS). (a) S-2 self-assembled from P1-NP2-P1 and P2-NP2-P2; (b) S-4 self-assembled from P1-NP4-P1 and P2-NP4-P2.

\subsection{Conclusion}

A novel type of self-assembled fibrous nanoparticle scaffolding system was demonstrated that employs peptide-functionalized polymeric nanoparticles that 
self-assemble into continuous 'nanoparticle-fibers' in aqueous solution. This type of nanoparticle scaffold possesses all the advantages of nanoparticles' abilities to contain and control the release of active ingredients (demonstrated here with model hydrophobic and hydrophilic drugs) with the ability of peptides to assemble into controlled 1D, 2D, and 3D structures. Combining these capabilities into a single "device" allows the simultaneous incorporation of multiple drugs, each with the desired quantity and release rate appropriate for that drug, along with the ability to obtain a uniform distribution of these drugs in a scaffolding system, or if desired a non-uniform distribution, and couples these abilities with the flexibility of hydrogel scaffold if desired. The porosity of the nanoparticle scaffolds, and the release rate of incorporated drugs, can be controlled by adjusting polymeric nanoparticle size and composition. This can be accomplished by any controlled polymerization route, but was done hereby RAFT polymerization. The versatility of this fundamental technique can be expanded to other biocompatible amphiphilic copolymers as long as it contains reactive end groups that are able to couple with ionic complementary peptides. This system can also co-assemble with host ionic complementary peptides to form nanoparticle-peptide composite gel scaffolds, which can further broaden the applications of polymeric controlled drug delivery system and peptide nanofiber scaffolds for tissue engineering. Our studies show that optimization of the scaffold surface is required to improve cell adhesion to nanoparticle surface s, which can be accomplished by immobilizing cell adhesion ligands on polymeric nanoparticle surfaces. In this work we also noted that the designer peptide self-assembly is easily converted into an amorphous aggregate after washing with aqueous solution, which was due to an overly water-soluble peptide structure, so alternative designer peptides can be designed with less solubility to increase the mechanical stability and lower the critical concentration to form stable self-assembled peptide nanofibers with enhanced mechanical strength. 


\section{Main Findings in This Chapter:}

Amphiphilic triblock copolymers were synthesized by RAFT polymerization using BDAT as CTA. These amphiphilic copolymers were shown to self-assemble into core-shell nanoparticles, tobe functionalized with ionic complementary peptides (P1 and $\mathrm{P} 2$ ), and then to undergo furtherself-assembly into fibrous nanoparticle scaffolds with porosity and morphology depending on nanoparticle size. The nanoparticle scaffolds were prepared with different model drugs that were hydrophobic and hydrophilic, and that could be loaded into different sets of peptide-functionalized nanoparticles and self-assembled into nanoparticle scaffolds. Controlled release tests showed simultaneous release with different releasing rates from the scaffolding system, and that the releasing rate of these model drugs were effectively governed by particle size and composition. The peptide-functionalized nanoparticles can also co-assemble with host ionic complementary peptides (P1 and P2) to form composite peptide hydrogels, showing uniform distribution of nanoparticles in peptide hydrogel scaffolds with microscale diameter and in a fiber length that is hundreds ofmicromemters long, which is much larger scale than traditional peptide nanofiber scaffolds. The designed nanoparticle scaffolds with different particle size and shell composition demonstratedexcellent biocompatibility with NIH3T3 fibroblast cell lines and also possessed excellent stability in cell media throughout the cell test period.

\section{Acknowledgements:}

The authors thank the Department of Chemistry for financial support of this project, and Dr. Dave Chesney for training to use HPLC, Dr. Qili Hu for training to use fluorescence spectrometer, Mr. Jerry Anzalone of the Department of Material Science and Engineering for training in the use of optical microscopy analysis. 


\section{References:}

[1]. Ruth R. Chen, David J. Mooney, Polymeric growth factor delivery strategies for tissue engineering, Pharmaceutical Research, 2003,20 (8), 1103-1112.

[2]. M. J. Whitaker, R. A. Quirk, S. M. Howdle and K. M. Shakesheff, Growth factor release from tissue engineering scaffolds, Journal of Pharmacy and Pharmacology, 2001, 53: $1427-1437$.

[3]. Aurore Schneider, Jonathan A. Garlick and Christophe Egles, Self-assembling peptide nanofiber scaffolds accelerate wound healing, PLoS ONE, 2008, (1), e1410.

[4]. Jason A Burdick, Mariah N Mason, Adrian D Hinman, Kevin Thorne, Kristi S Anseth, Delivery of osteoinductive growth factors from degradable PEG hydrogels influences osteoblast differentiation and mineralization, Journal of Controlled Release, 2002, 83(1),53-63.

[5]. Niancao Chen, Zhaoyang Zhang, BoonchoySoontornworajit, Jing Zhou, Yong Wang, Cell adhesion on an artificial extracellular matrix using aptamer-functionalized PEG hydrogels, Biomaterials, 2012, 33(5), 1353-1362.

[6]. Christine T. Schwall and Ipsita A. Banerjee, Micro- and Nanoscale Hydrogel Systems for Drug Delivery and Tissue Engineering, Materials 2009, 2, 577-612.

[7]. Yihong Gong, Lijuan He, Jun Li, Qingliang Zhou, Zuwei Ma, ChangyouGao, JiacongShen, Hydrogel-filled polylactide porous scaffolds for cartilage tissue engineering, Journal of Biomedical Materials Research Part B: Applied Biomaterials, 2007, 82B(1), 192-204.

[8]. Steven M. Jay, Benjamin R. Shepherd,James P. Bertram, Jordan S. Pober and W. Mark Saltzman, Engineering of multifunctional gels integrating highly efficient growth factor delivery with endothelial cell transplantation, PNAS, 2010,107(10), 4669-4674.

[9]. Jenna M. Shapiro and Michelle L. Oyen, Hydrogel composite materials for tissue engineering scaffolds, JOM, 2013,65(4), 505-516. 
[10]. Silviya P. Zustiak, Yunqian Wei, and Jennie B. Leach. Protein-Hydrogel Interactions in Tissue Engineering: Mechanisms and Applications, Tissue Engineering Part B: Reviews. 2013, 19(2): 160-171.

[11]. Xiaoran Li, Zhifeng Xiao, Jin Han, Lei Chen, Hanshan Xiao, Fukai Ma, XianglinHou, Xing Li, Jie Sun, Wenyong Ding, Yannan Zhao, Bing Chen, Jianwu Dai, Promotion of neuronal differentiation of neural progenitor cells by using EGFR antibody functionalized collagen scaffolds for spinal cord injury repair, Biomaterials, 2013, 34(21), $5107-5116$.

[12]. Samit K. Nandi, BiswanathKundu, DebabrataBasu, Protein growth factors loaded highly porous chitosan scaffold: A comparison of bone healing properties, Materials Science and Engineering: C, 2013, 33(3), 1267-1275.

[13]. Marta Michalska, KatarzynaKaplinska, MarekMirowski, AndrzejBodek, Kazimiera H. Bodek,Evaluation of the use of fibrin and microcrystalline chitosan membranes as carriers for transforming growth factor Beta-1, Journal of applied polymer science, 2013, 127(5), 3506-3513.

[14]. Ran Ito, Naoki Morimoto, LiemHieu Pham, TsuguyoshiTaira, Katsuya Kawai, and Shigehiko Suzuki, Efficacy of the controlled release of concentrated platelet lysate from a collagen/gelatin scaffold for Dermis-Like tissue regeneration, Tissue Engineering Part A. 2013, 19(11-12): 1398-1405.

[15]. Matthew J. Caicco, Michael J. Cooke, Yuanfei Wang, AnupTuladhar, Cindi M. Morshead, Molly S.Shoichet, A hydrogel composite system for sustained epi-cortical delivery of Cyclosporin A to the brain for treatment of stroke, Journal of Controlled Release 166 (2013) 197-202.

[16]. Shuguang Zhang, FabrizioGelain and Xiaojun Zhao, Designer self-assembling peptide nanofiber scaffolds for 3D tissue cell cultures, Seminars in Cancer Biology, 2005, $15,413-420$. 
[17]. Zhang S, Holmes TC, Lockshin C, Rich A. Spontaneous assembly of a self-complementary oligopeptide to form a stable macroscopic membrane. Proc. Natl. Acad. Sci. U. S. A. 1993; 90: 3334-38.

[18]. Shuguang Zhang, FabrizioGelain and Xiaojun Zhao, Designer self-assembling peptide nanofiber scaffolds for 3D tissue cell cultures, Seminars in Cancer Biology, 2005, $15,413-420$.

[19]. Zhang S, Lockshin C, Cook R, Rich A. Unusually stable beta-sheet formation of an ionic self-complementary oligopeptide. Biopolymers, 1994, 34, 663-72.

[20]. Ramachandran S, Tseng Y, Yu B Y. Repeated rapid shear-responsiveness of peptide hydrogels with tunable shear modulus.Biomacromolecules2005;6: 1316-21.

[21]. Nagai Y, Unsworth LD, Koutsopoulos S, Zhang S. Slow release of molecules in self assembling peptide nanofiber scaffold. J. Controlled Release 2006;115:18-25.

[22]. Koutsopoulos S, Unsworth LD, Nagai Y, Zhang S. Controlled release of functional proteins through designer self-assembling peptide nanofiber hydrogel scaffold. Proc. Natl. Acad. Sci. U. S. A. 2009;106:4623-8.

[23]. Horii A, Wang X, Gelain F, Zhang S. Biological designer self-assembling peptide nanofiber scaffolds significantly enhance osteoblast proliferation, differentiation and 3-D migration. PLoSOne 2007;2:e190.

[24]. Gelain F, Unsworth LD, Zhang S. Slow and sustained release of active cytokines from self-assembling peptide scaffolds. J. Controlled Release 2010;145:231-9.

[25]. Galler KM, Aulisa L, Regan KR, D'Souza RN, Hartgerink JD. Self-assembling multidomain peptide hydrogels: designed susceptibility to enzymatic cleavage allows enhanced cell migration and spreading. J. Am. Chem. Soc. 2010;132:3217-23.

[26]. Xiaochu Ding, JagadeeshJanjanam, AshutoshTiwari, Martin Thompson, Patricia A. Heiden, Studies of Novel Self-Assembly Structures from Peptide-Functionalized Polymeric Micro- and Nanoparticles for Tissue Engineering, 2013, in submission. 
[27].DaweiZou, Zouxiu Tie, Chunmei Lu, Meng Qin, Xiaomei Lu, Mu Wang, Wei Wang, P. Chen, Effects of hydrophobicity and anions on self-assembly of the peptide EMK16-II, Biopolymers, 2010, 93(4), 318-329.

[28]. Boyer C, Stenzel MH, Davis TP. Building nanostructures using RAFT Polymerization.J.of Polymer Science Part A-Polymer Chemistry 2011;49(3):551-595.

[29] P. Chen, Self-assembly of ionic-complementary peptides: a physicochemical viewpoint. Colloids and Surfaces A: Physicochemical and Engineering Aspects, 2005,261(1-3),3-24. 


\title{
Efficient One-Pot Synthesis and Loading of Self-Assembled Amphiphilic
}

\section{Chitosan Nanoparticles for Low-Leaching Wood Preservation}

\begin{abstract}
Xiaochu Ding ${ }^{1}$, Dana L. Richter ${ }^{2}$, Laurent M. Matuana ${ }^{3}$, and P.A. Heiden ${ }^{1 *}$
${ }^{1}$ Department of Chemistry and ${ }^{2}$ School of Forest Resources and Environmental Science, Michigan Technological University, Houghton, Michigan 49931

The initial idea of this chapter was conceived by Dr. Heiden. Theresearchand experimental designs were by Mr. XiaochuDing, except for the soil jar decay testing, which was conducted by Dr. Dana L. Richter in his lab with the assistance of Xiaochu Ding, and the SEM micrographs, that were taken by Mr. Owen Mills.Dr. L. Matuana assisted with data analysis. This research was funded by the United States Environmental Protection Agency Science to Achieve Results (STAR) Program, Grant Number GR832371.
\end{abstract}

Mr. Xiaochu Ding

Department of Chemistry

Michigan Technological University
Dr. Dana L. Richter

School of Forest Resources and Environmental Science

Michigan Technological University

Dr. Laurent M. Matuana

Dr. Patricia A. Heiden*

Department of Packaging

Department of Chemistry

University of Michigan 


\section{Chapter 4}

Efficient One-Pot Synthesis and Loading of Self-Assembled Amphiphilic Chitosan Nanoparticles for Low-Leaching Wood Preservation

Xiaochu Ding ${ }^{1}$, Dana L. Richter ${ }^{2}$, Laurent M. Matuana ${ }^{3}$, and P.A. Heiden ${ }^{1 *}$

${ }^{1}$ Department of Chemistry and ${ }^{2}$ School of Forest Resources and Environmental Science, Michigan Technological University, Houghton, Michigan 49931

Corresponding author. Patricia A. Heiden, Xiaochu Ding, Tel.: +1 9064873452

Email address: paheiden@mtu.edu, xding@mtu.edu

This paper is published in Carbohydrate Polymers, 86(1), 58-64 (2011). 


\begin{abstract}
A simple, one-step and one-pot method was used to synthesize amphiphilic self-assembling chitosan-g-PMMA nanoparticles $(\sim 100 \mathrm{~nm}$ diameter by SEM, but $\sim 150-200 \mathrm{~nm}$ in water by DLS), containing $\sim 25-28 \mathrm{wt} \%$ ( $\sim 82-93 \%$ capture efficiency) of the fungicide tebuconazole. The matrix composition was selected to be environmentally low impact, while the nanoparticle preparation conditions were designed to ensure the nanoparticles sufficiently small to be able to penetrate the pit-pores of solid wood. These nanoparticles were delivered into southern pine sapwood blocks at target fungicide retentions of $0.2,0.4$ and $0.8 \mathrm{~kg}$ tebuconazole $/ \mathrm{m}^{3}$ wood. SEM analysis of a $19 \times 19 \times 455$ $\mathrm{mm}$ nanoparticle-treated wooden stake confirmed penetration throughout the interior of the treated stake. Leaching studies confirmed that biocide introduced into sapwood via nanoparticle carriers leached only about $9 \%$ as much fungicide as solution-treated controls, while soil jar tests showed the nanoparticle-treated wood blocks effectively protected the wood from biological decay when tested against G. trabeum, a brown rot fungus.
\end{abstract}

Keywords: controlled release, chitosan-g-PMMA nanoparticle, amphiphilic nanoparticle, core-shell structure, tebuconazole, biocide leach 


\subsection{Introduction}

Amphiphilic block and graft copolymers are routinely used to prepare self-assembling core-shell nanoparticles. Such copolymers are readily prepared from synthetic polymers, biopolymers, or combinations of both (1-8). Polysaccharides and proteins are well-suited for preparing grafted amphiphilic copolymer nanoparticles because of the large number of polymerizable groups they possess along their backbone.

Chitosan is possibly the most commonly selected polysaccharide for grafted amphiphilic copolymer nanoparticles. Its repeat units bear either a primary amine group or an amide group if that unit is not hydrolyzed. The large number of amine groups can be utilized in either grafting-to or grafting-from reactions. A recent review describes the synthesis, properties, and uses of many different chitosan amphiphiles $(\mathbf{8 , 9})$. One "grafting from" reaction uses these amines to react with peroxides under mild conditions

yielding amine radicals that can efficiently react with hydrophobic monomers to form amphiphilic core-shell nanoparticles (1).

Chitosan's biocompatibility, biodegradability, and antimicrobial activity are why it is employed in many biomedical and cosmetic applications. A recent review describes the synthesis, properties, and uses of many different chitosan amphiphiles (8). However, high-value applications still account for most nanoparticle uses, improvements in technology as well as consumer trends are allowing nanoparticle uses to penetrate commodity areas as well. For example, one recent publication describes amphiphilic chitosan micelles for the controlled release of rotenone (9). However, that used a multi-step synthesis and the encapsulation efficiency was low. Another report, from 2003 described controlled release of agrochemicals from chitosan (10), but those were microparticles.

The objective of this project was to study the beneficial effects of using nanoparticles in wood preservation. We had previously studied controlled release nanoparticles in solid 
wood, prepared using synthetic polymers under dilute conditions in a solvent-displacement route (11-13). The nanoparticle-treated wood was tested against both a brown and white rot fungus, and biological efficacy compared well with the solution-treated controls. At that time no work was done to test the biocide leaching.

The rationale for employing controlled release nanoparticles to deliver organic biocides into solid wood was the hypothesis that a controlled biocide release would maintain an effective protection of the solid wood and might also reduce biocide leaching into the environment. The significance of reducing biocide leaching from treated wood is that when biocide leaches from wood it leaves the wood less well-protected from biological attack, and the leached biocide can have detrimental effects on the environment into which it is released. If less biocide is lost to the environment, then potentially the wood could be effectively protected with less biocide, which is beneficial to both the environment and to the cost of the preserved wood. In this application solid nanoparticles are favored over liquid micelles, which are more easily delivered into wood than solid particles, because of the desire to control the biocide release and to avoid the use of surfactant. The amphiphilic design is preferred because this allows a hydrophobic core composition to be used to manipulate the release rate while the shell can be hydrophilic to give a stable suspension in water. The nanometer size, preferably below $150 \mathrm{~nm}$, is required because of the anatomy of solid wood, where the small size is required to penetrate the pit pores to enter into the wood interior.

Our recent study supported the hypothesis of reduced biocide leach using gelatin-g-PMMA nanoparticles (1), but ungrafted gelatin complicated analysis of the data, and the gelatin may have promoted biological decay within the wood. The purpose of this paper is to prove the hypothesis that decreased biocide leach occurs by use of controlled-release nanoparticles, to quantify the decrease in biocide leaching, to show that wood preservation is not decreased, and to confirm nanoparticle penetration into 
wood interior on larger wood specimens than those used in standard soil jar studies. Here we prepared fungicide-containing chitosan-g-PMMA core-shell nanoparticles using the grafting approach outlined in Scheme 4.1. Chitosan was selected as the shell material despite the fact that the NPs could be prepared at only $\sim 2-5 \mathrm{wt} \%$ polymer solids in an aqueous medium containing acetic acid, because it was speculated that chitosan might enhance wood preservation, while gelatin, which can be used to prepare nanoparticles by this approach at $10 \mathrm{wt} \%$ solids appeared to contribute to biological decay and made analysis more difficult.

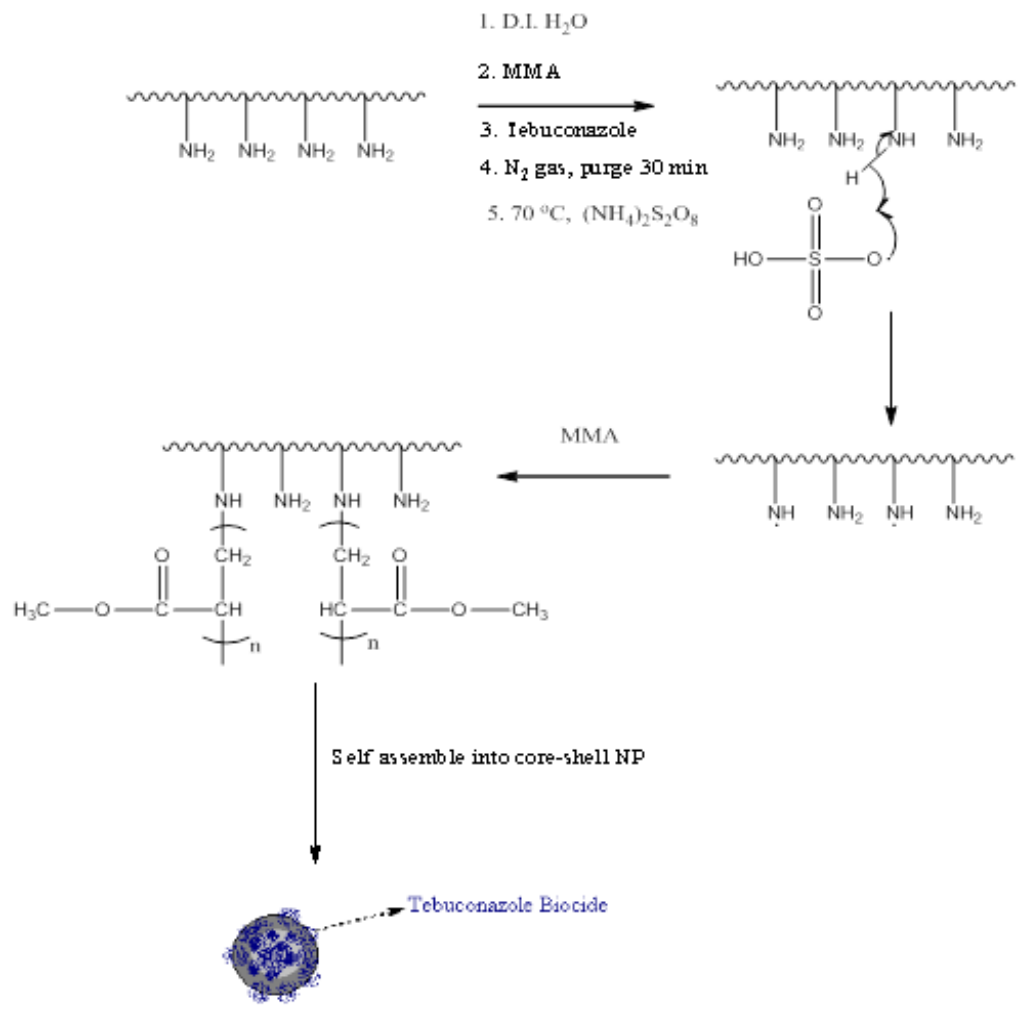

Scheme 4.1. Synthetic route producing self-assembled chitosan-g-PMMA nanoparticles. 


\subsection{Experimental Part}

\subsubsection{Materials}

Chitosan ( $\sim 70 \%$ deacetylation) from crab shell was donated by Cochin University of Science and Technology (Cochin, India). Tebuconazole fungicide was donated by Lanxess Corporation (Pittsburg, PA). Methyl methacrylate (MMA, 99.0\%) and 2-hydroxyethylmethacrylate (HEMA, 97\%) were from Sigma Aldrich (Milwaukee, USA) and were distilled prior to use. Ammonium persulfate (APS, 95\%) and benzophenone (99\%) were from Mallinckrodt Chemical Works (St. Louis, USA) and Sigma Aldrich, and were used as received. Fungal tests used Gloeophyllum trabeum (ATCC 11539), a basidomycete brown rot wood decay fungus. Wood blocks were 19x19x19 mm, unless otherwise noted, and were cut from southern pine sapwood in the MTU School of Forest Resources and Environmental Science.

\subsubsection{Preparation of Nanoparticles}

Nanoparticles were prepared using a modification of the methods reported by Li (14) and Qian (15). Briefly, chitosan was dissolved in deionized water ( $\left.\mathrm{DI} \mathrm{H}_{2} \mathrm{O}\right)$ containing acetic acid (0.67 $\mathrm{g}$ acetic acid/g chitosan). For example, $0.5 \mathrm{~g}$ chitosan was dissolved in $0.43 \mathrm{wt} \% \mathrm{AcOH}$ to give $75 \mathrm{~mL}$ of solution at $50{ }^{\circ} \mathrm{C}$. The reaction solution was purged with nitrogen gas for $0.5 \mathrm{~h}$ prior to the addition of MMA (MMA to Chitosan 2:1 w/w). The total concentration of chitosan and MMA ranged from 1-5 \% w/w. Tebuconazole (30 $\mathrm{w} \%$ based on the combined mass of chitosan and MMA) was dissolved in about $5 \mathrm{~mL}$ of acetone and then added dropwise to the reaction solution under magnetic stirring. After mixing for $10 \mathrm{~min}$, the reaction temperature was increased to $70{ }^{\circ} \mathrm{C}$. APS $(0.037 \% \mathrm{w} / \mathrm{v})$, dissolved in a small amount of deionized water, was then added to initiate the grafting reaction. The reaction was kept at $70{ }^{\circ} \mathrm{C}$ with magnetic stirring at $400 \mathrm{rpm}$ for $24 \mathrm{~h}$. This procedure was also used to prepare nanoparticles without tebuconazole by skipping the tebuconazole addition step. The yield of chitosan-g-PMMA NPs without tebuconazole 
ranged from $\sim 94-99 \%$ with a grafting efficiency of $\sim 74-87 \%$.

\subsubsection{Nanoparticle Size}

The particle size of as-made nanoparticles was determined (in aqueous suspension) by Dynamic Light Scattering (Coulter NP4 Plus, Beckman Coulter, Fullerton, CA) and by SEM (Shimadzu QP5050A, Shimadzu Corporation, Germany) using freeze-dried nanoparticles.

\subsubsection{Nanoparticle Composition}

Nanoparticle composition (without tebuconazole) was determined by gravimetric analysis. The as-made nanoparticle suspension was collected, gently heated to evaporate most solvent, and then vacuum dried at $50{ }^{\circ} \mathrm{C}$ for $48 \mathrm{hrs}$. The dried NPs were extracted with $3 \times 15 \mathrm{~mL}$ of chloroform to separate any PMMA homopolymer, and then $3 \times 20 \mathrm{~mL}$ of warm deionized water containing $0.67 \mathrm{~g}$ acetic acid to separate any ungrafted chitosan. The extracts were vacuum dried at $50{ }^{\circ} \mathrm{C}$ for $48 \mathrm{hrs}$ to obtain the mass of PMMA and ungrafted chitosan. The residual mass was chitosan-g-PMMA.

\subsubsection{Tebuconazole Content in Nanoparticles}

An aliquot of the tebuconazole-containing nanoparticle suspension was weighed, gently heated to remove most solvent, and then vacuum dried at $50{ }^{\circ} \mathrm{C}$ for $48 \mathrm{hrs}$ to get the initial mass of tebuconazole-containing NPs. The tebuconazole component was extracted from the dried NPs using $3 \times 15 \mathrm{~mL}$ of ethanol, and the combined extracts were then vacuum dried at $40{ }^{\circ} \mathrm{C}$ to get the tebuconazole mass. The solid extracts were confirmed to be pure tebuconazole by ${ }^{1} \mathrm{H}$ NMR. The calculation of the actual content of tebuconazole in NP suspension and the needed amount of the as-made NPs suspension to treat wood blocks by pressure-treatment is shown in equations (1) and (2): 
(1) Tebuconazole $\%=\frac{\text { Mass }(\text { Teb. })}{\text { Mass(suspension })} \times 100 \%$

(2) Mass $($ suspension $)=\frac{\operatorname{Re} \text { tention }(T \arg \text { et }) \times V(\text { woodblocks })}{\text { Tebuconazole } \%}$

\subsubsection{Delivery Efficiency into Wood Blocks}

Wood blocks were submerged in alcohol for $24 \mathrm{hrs}$ to remove some soluble extractives and wood sawdust near the surface pores, which would interfere with GC-MS analysis and gravimetric analysis. Then wood blocks were treated with NP suspensions in accordance with procedures given as the standard method in Wood Pressure Treatment (AWPA E11-97) (16). The quantity of as-made nanoparticle suspension needed to deliver target retentions of $0.2,0.4$ or $0.8 \mathrm{~kg}$ tebuconazole $/ \mathrm{m}^{3}$ wood, assuming quantitative delivery, was taken and diluted to $90 \mathrm{~mL}$. The $90 \mathrm{~mL}$ volume was required to sufficiently cover 6 wood blocks having dimensions of 19x19x19 mm when these blocks were placed in a beaker. The wood blocks were covered with a plastic mesh and aluminum blocks to keep them submerged throughout the treatment process. The beaker was then transferred into the pressure cylinder and subjected to a pressure treatment consisting of a partial vacuum of less than $25 \mathrm{mmHg}$ for $0.5 \mathrm{~h}$, followed by pressurization to $100 \mathrm{psi}$ for $1 \mathrm{~h}$. Specimens were removed, and the remaining suspension was transferred into a pre-weighed aluminum dish and heated to dryness to determine the NP mass that was not absorbed by wood. Because some extractives are absorbed into the suspension during the wood treatment, a measurable residue also results from treating wood "blanks" with water. Therefore the measured mass from wood "blanks" is used to adjust the undelivered mass from the NP-treated wood specimens. Therefore the delivery efficiency percentage is calculated as:

(3) $\%$ Delivery $=[($ dry NP mass in wood - average mass loss from blanks $) /$ initial dry NP mass] x 100 


\subsubsection{Leaching Tests}

The leaching test was performed according to The American Wood Preservers Association Standard (AWPA E11-97) (16). The leachate was collected in a beaker and heated at $80{ }^{\circ} \mathrm{C}$ to remove water, and then acetone was added to the dried leachate. The beaker was sealed for $3 \mathrm{hrs}$ to completely extract the tebuconazole from the leachate. Then the acetone solution was transferred to a volumetric flask and a known quantity of benzophenone was added to the solution for use as an internal standard to allow a quantitative analysis by GC-MS.

\subsubsection{GC-MS Analysis}

AGas Chromatograph/Mass Spectrometer (Shimadzu 5050A, Shimadzu Corporation, Germany) equipped with a programmed-temperaturevaporizer was used to measure the amount of leached tebuconazole collected as described. MS withelectron-impact (EI) ionization (electronenergy $70 \mathrm{eV}$ ) was performed in selectedion monitoring (SIM) mode. The injection temperature and volume were $280{ }^{\circ} \mathrm{C}$ and $1 \mu \mathrm{L}$, which was $100 \%$ delivered into chromatography column under a flow rate of $1 \mathrm{~mL} \mathrm{He} / \mathrm{min}$ as the carrier gas. The oven temperature, initially at $50{ }^{\circ} \mathrm{C}$, was raised to $100{ }^{\circ} \mathrm{C}$ and held for 3 min to remove solvent. Then the MS detector began to identify the analyte while ramping the temperature at $10{ }^{\circ} \mathrm{C} / \mathrm{min}$, and finally holding $5 \mathrm{~min}$ at $325^{\circ} \mathrm{C}$ to remove possible residues in column. The quantitative analysis was based on the peak areas from mass chromatograph. The internal response factor (IRF) was firstly identified by a standard solution with a known amount of tebuconazole (TEB) and benzophenone (BEN). The IRF was calculated according to equation 4:

(4) $I R F=\frac{\operatorname{Area}(B E N) \times \operatorname{Amount}(T E B)}{\operatorname{Amount}(B E N) \times \operatorname{Area}(T E B)}$

After determining the IRF, the leached tebuconazole amount could be calculated based on the internal standard method according to equation 5 . 
(5) Amount $(T E B)=\frac{\operatorname{Area}(T E B) \times \operatorname{Amount}(B E N) \times I R F}{\operatorname{Area}(B E N)} \times V($ solution $)$

\subsubsection{Soil Jar Decay Test}

Treated southern pine wood blocks $(19 \times 19 \times 19 \mathrm{~mm})$ were dried at $40{ }^{\circ} \mathrm{C}$ for $24 \mathrm{~h}$. The mass was measured to $\pm 0.005 \mathrm{~g}$. All blocks were exposed to the brown rot fungus, Gloeophyllum trabeum ATCC 11539 for 12 weeks. Decay testing was done using American Wood Preservers Association (AWPA) testing method E-10-07, "Standard Method of Testing Wood Preservatives by Laboratory Soil Block Cultures”. 


\subsection{Results and Discussion}

\subsubsection{Study of Reaction Conditions}

NP diameter typically shows a strong dependency on the concentration of the medium in which they are made regardless of if they are made by reaction, solvent-displacement or precipitation. To be used as controlled release devices in solid wood an NP diameter below $150 \mathrm{~nm}$ was desired for good penetration into the wood interior. Consequently, the first study tested the effect of the reaction concentration on NP diameter (Fig.4.1). Because the NPs will be introduced into the wood in aqueous medium, the diameter of the water-swollen NPs will affect their ability to penetrate the wood interior. Figure 4.1 shows the particle size of the water-swollen NPs, measured by DLS, of chitosan-g-PMMA NPs (designated C2M, indicating that the polymer matrix was made using a mass ratio of 2 parts of MMA to 1 part chitosan) after $24 \mathrm{~h}$ of reaction at concentrations, based on polymer solids. The polymer solids concentration ranged from 1.5 up to $5 \mathrm{wt} \%$ in acidic $\mathrm{H}_{2} \mathrm{O}(0.43 \mathrm{wt} \% \mathrm{AcOH})$. The results show that a concentration of $2 \mathrm{wt} \%$ or less is required to afford nanoparticles with a water-swollen diameter below $200 \mathrm{~nm}$. The C2M water-swollen diameter was $\sim 167 \pm 56 \mathrm{~nm}$ and typically increased by $\sim 20-30 \mathrm{~nm}$ when prepared with biocide. Therefore, $2 \mathrm{wt} \% \mathrm{C} 2 \mathrm{M}$ in D.I. $\mathrm{H}_{2} \mathrm{O}$ was used as the maximum acceptable concentration for these studies, although the diameter was larger than desired. 


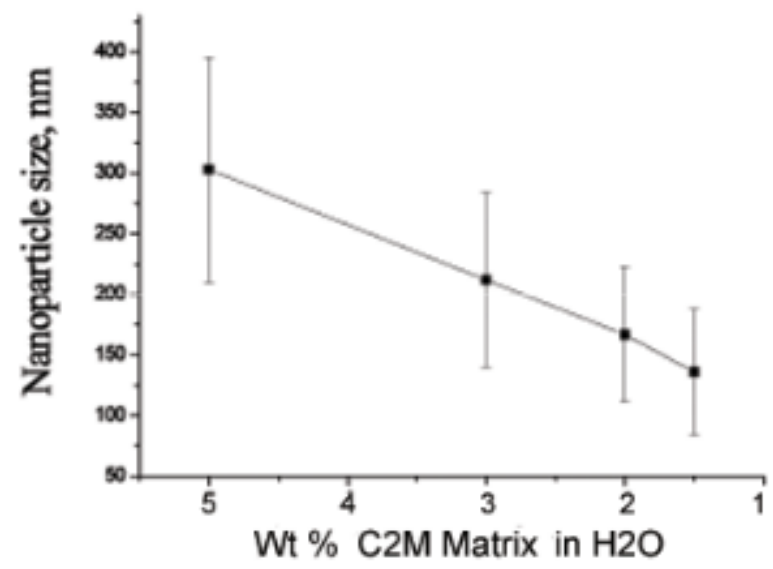

Figure 4.1. Particle size (by DLS) of C2M NPs as a function of reaction concentration.

The particle size can be decreased by increasing the protonation of the chitosan amine groups (by increasing acetic acid from $0.67 \mathrm{~g}$ to $2.0 \mathrm{~g} / \mathrm{g}$ chitosan). At either concentration of acetic acid the diameter of the NPs can be further decreased by sonication. For example, the final NP diameter of C2M NPs made at $0.67 \mathrm{~g}$ of acetic acid per gram of chitosan resulted in decreasing the diameter from $167 \pm 56 \mathrm{~nm}$ to $132 \pm 48 \mathrm{~nm}$. Interestingly, the NP diameter was not significantly altered by the ratio of the MMA to chitosan in the range we studied (1,2 or 3 parts MMA to 1 part chitosan). The NPs with 1, 2, and 3 parts of MMA to 1 part of chitosan (CM, C2M, and C3M respectively), prepared at $2 \mathrm{wt} \%$ increased by $\sim 22 \mathrm{~nm}$ as MMA content increased from 1:1 to 3:1 with respect to chitosan.

Interestingly, changing the ratio of MMA to chitosan does not significantly affect particle size, though it does increase the extent of PMMA homopolymer, as shown in Figure 4.2. The increase in the extent of homopolymerization is coincident to a slight decrease in the extent of grafting to chitosan. The amount of ungrafted chitosan is relatively constant. The ungrafted chitosan is probably the major contributor to the water-swollen diameter of the nanoparticles, which are typically $\sim 50-100 \mathrm{~nm}$ larger than the diameter observed by SEM. 


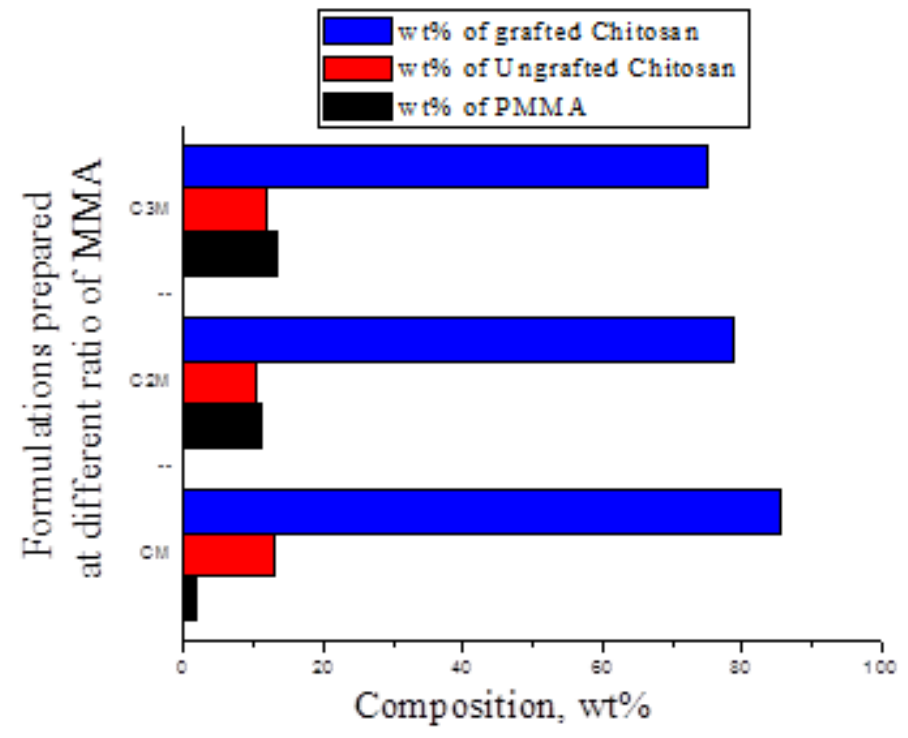

Figure 4.2. Composition of CM, C2M, and C3M NPs (142 $\pm 60,167 \pm 56$ and $166 \pm 51 \mathrm{~nm}$, respectively when prepared at $2 \mathrm{wt} \%$ in $75 \mathrm{~mL}$ D.I. $\mathrm{H}_{2} \mathrm{O}$ with $0.43 \mathrm{wt} \% \mathrm{AcOH}$ ).

\subsubsection{Characterization of Fungicide-Containing Nanoparticles}

In the second study the amount of tebuconazole introduced into the NPs and the effect of the tebuconazole on NP diameter was determined. In this work we arbitrarily selected a target tebuconazole quantity to be $30 \%$ of the final NP mass. Table 4.1 shows that the tebuconazole is incorporated at $\sim 93 \%$ yield. This high incorporation efficiency of the water-insoluble biocide supports the conclusion that this polymerization does not follow an emulsion polymerization route, but proceeds in a micellar route, leading to a final solid NP. The final suspension, after sonication, affords water-swollen C2M-tebuconazole NPs of $\sim 150 \mathrm{~nm}$.

One important advantage of using this radical grafting approach to amphiphilic copolymer NPs is that it is easy to "tune" the core composition from hydrophobic (only MMA) to increasingly hydrophilic by increasing the HEMA content. Even small changes in core hydrophilicity can alter the biocide release rate, which is diffusion controlled. 
Here the core composition was changed slightly by replacing $2 \mathrm{wt} \%$ of MMA monomer with HEMA monomer, giving C2MH NPs. The results, shown in Table 4.1, show the water-swollen $\mathrm{C} 2 \mathrm{MH}$-tebuconazole NPs have a larger diameter than that found for C2M-tebuconazole NPs (189 \pm 74 compared to $149 \pm 57 \mathrm{~nm})$. However, SEM found no significant difference in the average diameter of these NPs. The tebuconazole incorporation efficiency remained high, but was less than that obtained for C2M NPs, down from $\sim 93 \%$ to $\sim 82 \%$. This indicates that increasing the hydrophilicity of the system compromises the efficiency of the micelle "capture" of tebuconazole. Again, the high entrapment efficiency shows that this polymerization proceeds by a micellar route (17) rather than a typical emulsion route. That is, the biocide was present within a chitosan-stabilized micelle together with the acrylic monomer(s) at the start of the polymerization. Possibly the HEMA content allowed more tebuconazole to be retained in the shell and this was lost during NP isolation.

Table 4.1. NP size and tebuconazole content in $\mathrm{C} 2 \mathrm{M}$ and $\mathrm{C} 2 \mathrm{MH}$ NPs

\begin{tabular}{|ccccc|}
\hline Sample & $\begin{array}{c}\text { NP size,nm } \\
\text { (DLS/SEM) }\end{array}$ & $\begin{array}{c}\text { NPs } \\
(\mathbf{g})\end{array}$ & $\begin{array}{c}\text { Tebuconazole } \\
(\mathbf{g})\end{array}$ & $\begin{array}{c}\text { Tebuconazole, } \\
\mathbf{w t} \% \\
\text { (Theo./Actual) }\end{array}$ \\
\hline C2M-30\% Teb. & $149 \pm 57 / 100 \sim 150$ & 0.3500 & 0.0972 & $30.0 / 27.8$ \\
C2MH-30\%Teb. & $189 \pm 74 / 100 \sim 150$ & 0.5100 & 0.1257 & $30.0 / 24.6$ \\
\hline
\end{tabular}

\subsection{Wood Leaching and Wood Preservation}

\subsubsection{Nanoparticle delivery into wood blocks}

Aqueous suspensions of tebuconazole-containing C2M and C2MH NPs (C2M-Teb and $\mathrm{C} 2 \mathrm{MH}-\mathrm{Teb}$ ) were prepared and used to pressure-treat southern pine sapwood blocks according to the standard method of wood pressure treatment (AWPA E11-97) (16). The 
delivered NP mass was determined by measuring the undelivered mass, and the retention of tebuconazole in the wood blocks was calculated from the absorbed NP mass, as described in the Experimental. The results are shown in Table 4.2.

Table 4.2.Nanoparticle delivery and tebuconazole retention in wood blocks.

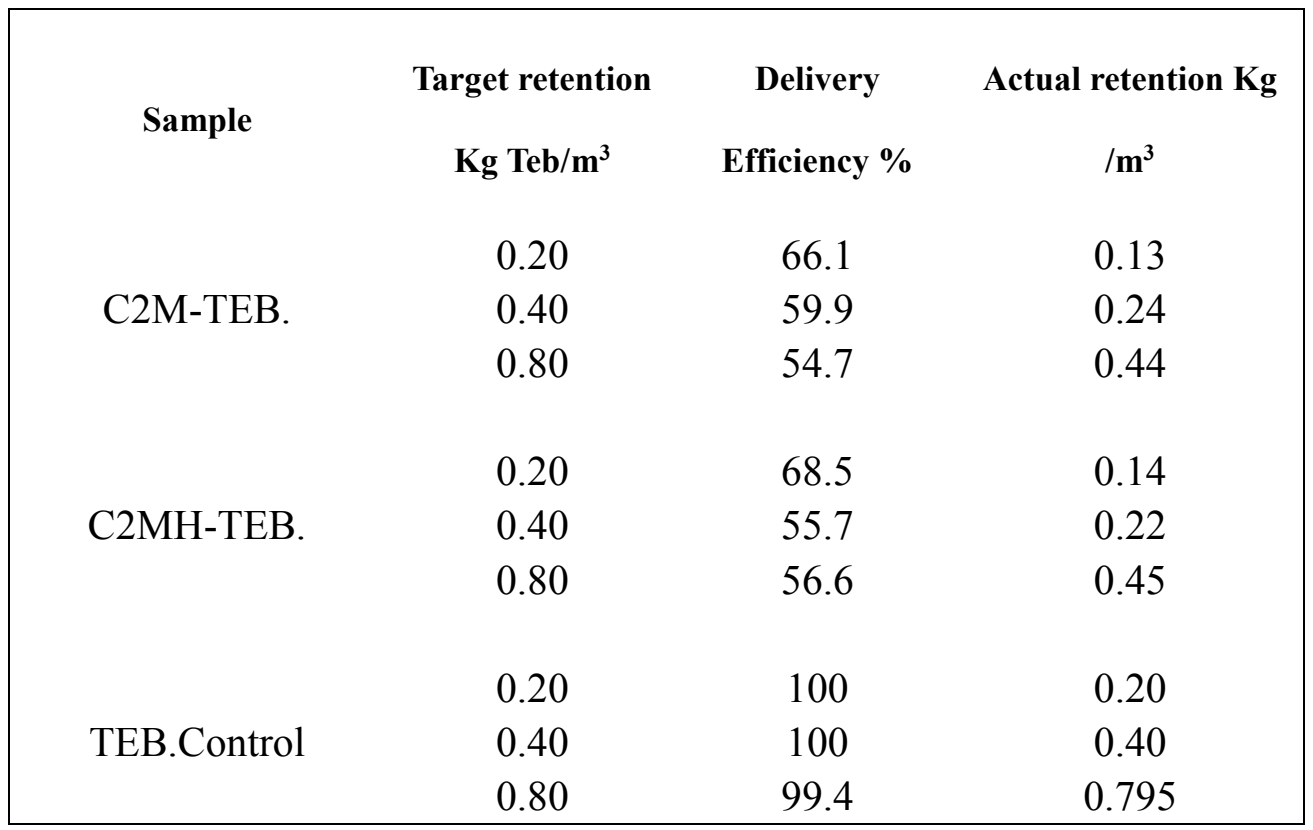

Previous studies with nanoparticle-treated southern pine sapwood (1,11-13) showed good biological efficacy and good penetration, but treatment was limited to $19 \times 19 \times 19$ $\mathrm{mm}$ blocks. One of the objectives of this work was to further prove good penetration of wood, using large wooden "field" stakes $(19 \times 19 \times 455 \mathrm{~mm})$. Several stakes were treated and sectioned to verify penetration of the wood interior is achieved even on larger wooden parts.

Figure 4.3 a shows an image of a treated field stake. Despite NP aggregates reducing delivery efficiency, good penetration of the wood stake was achieved as shown by FESEM images (Fig. $4.3 \mathrm{~b}$ and $4.3 \mathrm{c}$ ) showing interior sections. Figure $4.3 \mathrm{~b}$ shows a region deep in the interior of the wood stake while Figure $4.3 \mathrm{c}$ shows a section nearer 
the surface.

(a)

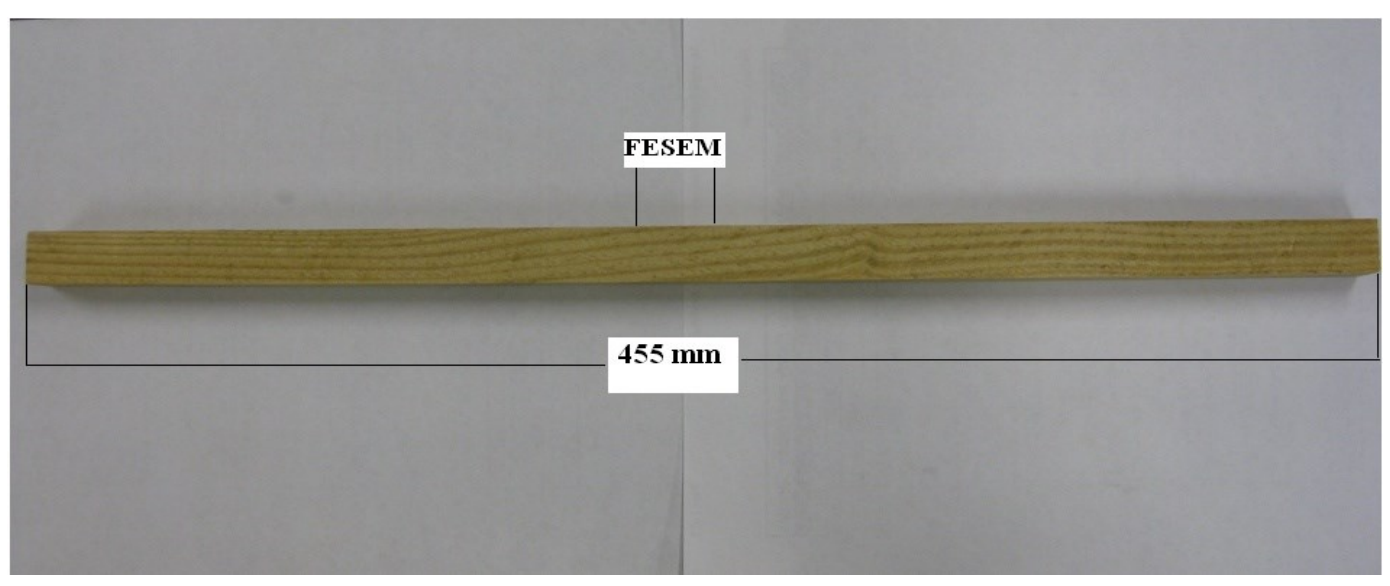

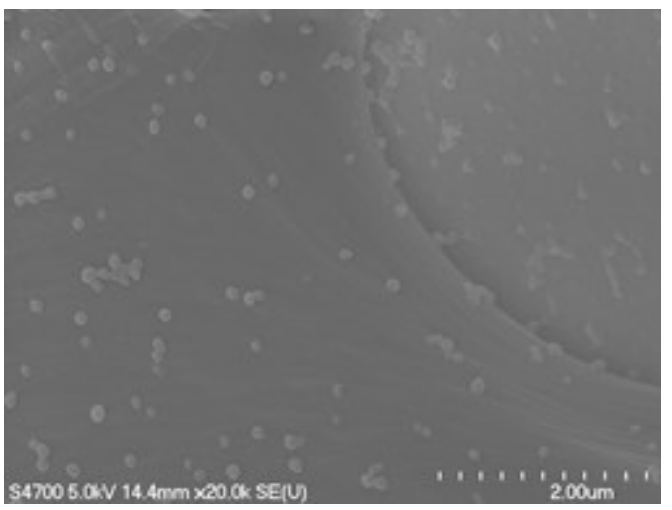

(b)

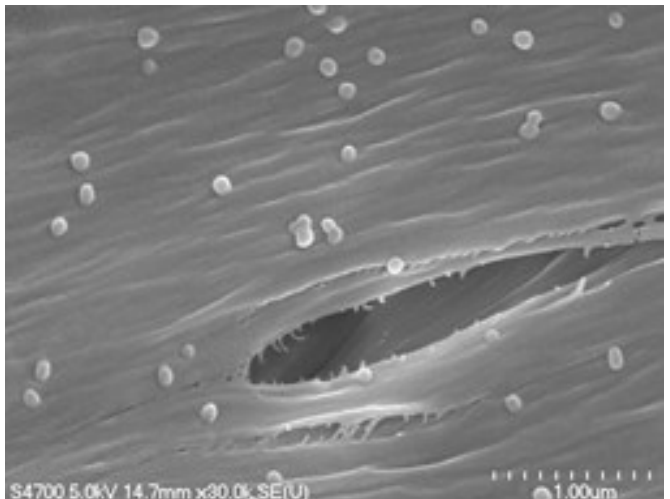

(c)

Figure 4.3. A wooden stake after NP treatment is shown, with a central section indicated (a, top); after the indicated central section is cut, then specimens suitable for FESEM are taken from two locations, one deep in the interior (b, bottom left) and from near the surface (c, bottom right).

\subsubsection{Leaching from wood blocks}

The primary purpose of this work was to prove the hypothesis that use of controlled-release biocide-containing NPs will reduce biocide leaching without compromising wood preservation. Wood blocks were treated to give theoretical 
biocide-retentions of $0.2,0.4$, and $0.8 \mathrm{~kg}$ tebuconazole $/ \mathrm{m}^{3}$ sapwood. Figure 4.4a-b shows the measured biocide leach from solution-treated tebuconazole controls with leach from wood treated with C2M-TEB and C2MH-TEB NPs at theoretical loadings of 0.4 and 0.8 $\mathrm{kg} / \mathrm{m} 3$ wood respectively. In both cases the solution-treated control leached significantly more tebuconazole than the nanoparticle-treated wood, and the C2MH-TEB nanoparticle-treated wood leached more tebuconazole than the C2M-TEB nanoparticle-treated wood.

Based on the cumulative leach the C2MH-TEB treated wood typically leached about two to three times the amount of tebuconazole as the C2M-TEB treated wood. Because the retentions of the $\mathrm{C} 2 \mathrm{MH}-\mathrm{TEB}$ and $\mathrm{C} 2 \mathrm{M}-\mathrm{TEB}$ treated sapwood were similar the leaching data are comparable with each other, but not with the solution-treated specimens. Therefore, Figure $4.4 \mathrm{c}$ re-plots the leach data for TEB-solution treated wood with the nanoparticle-treated wood, where the actual retentions are all close to $0.4 \mathrm{~kg} / \mathrm{m}^{3}$. Figure 4.4c plots the leach from a TEB-solution treated wood with an actual tebuconazole content of $0.4 \mathrm{~kg} / \mathrm{m}^{3}$, on the same axes as the C2MH-TEB and C2M-TEB nanoparticle-treated wood systems $\left(0.8 \mathrm{~kg} / \mathrm{m}^{3}\right.$ target retention) with actual retentions of 0.45 and $0.44 \mathrm{~kg} / \mathrm{m}^{3}$ respectively. Despite the nanoparticle-treated wood having higher tebuconazole retentions than the solution-treated control, it releases $\sim 2300 \mu \mathrm{g}$ of tebuconazole compared to $\sim 200 \mu \mathrm{g}$ for the C2M-TEB treated wood, and yet, which means that the C2M-TEB treated-wood leached less than $9 \%$ of the amount of biocide compared to the solution control, and, as the next section shows, did not sacrifice wood preservation based on results from a soil jar test. 


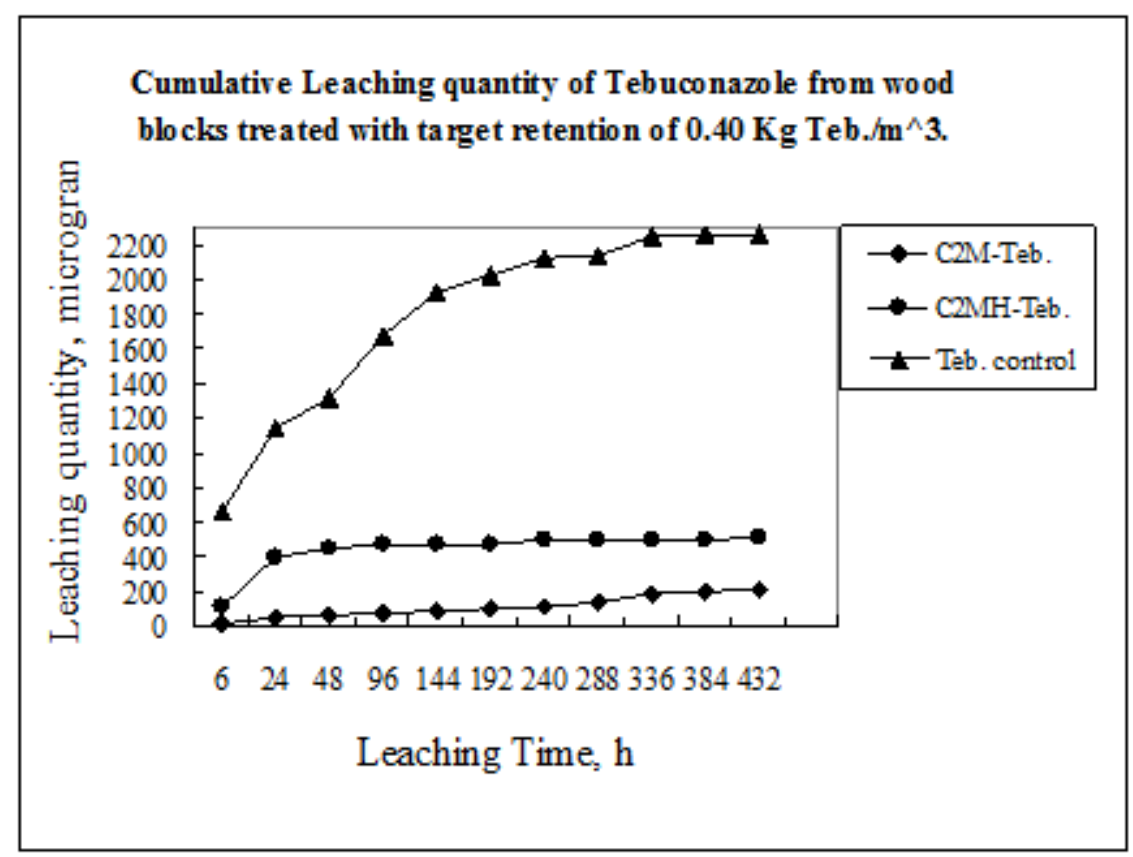

(a)

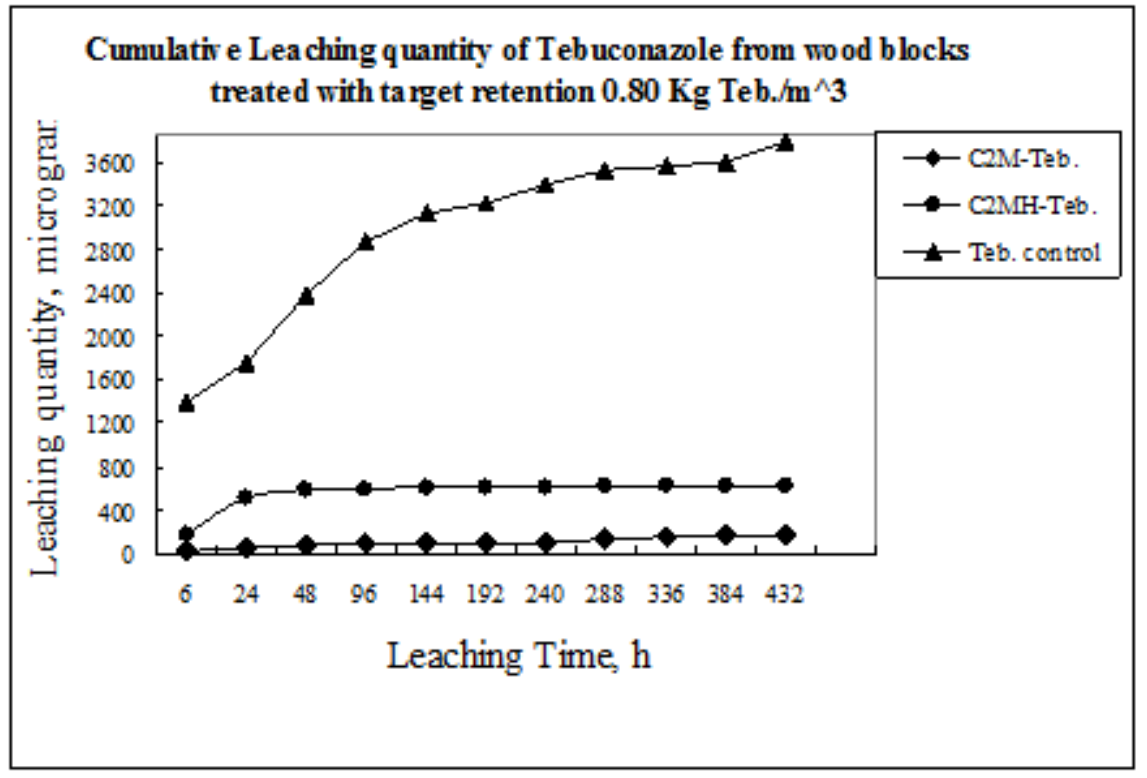

(b) 


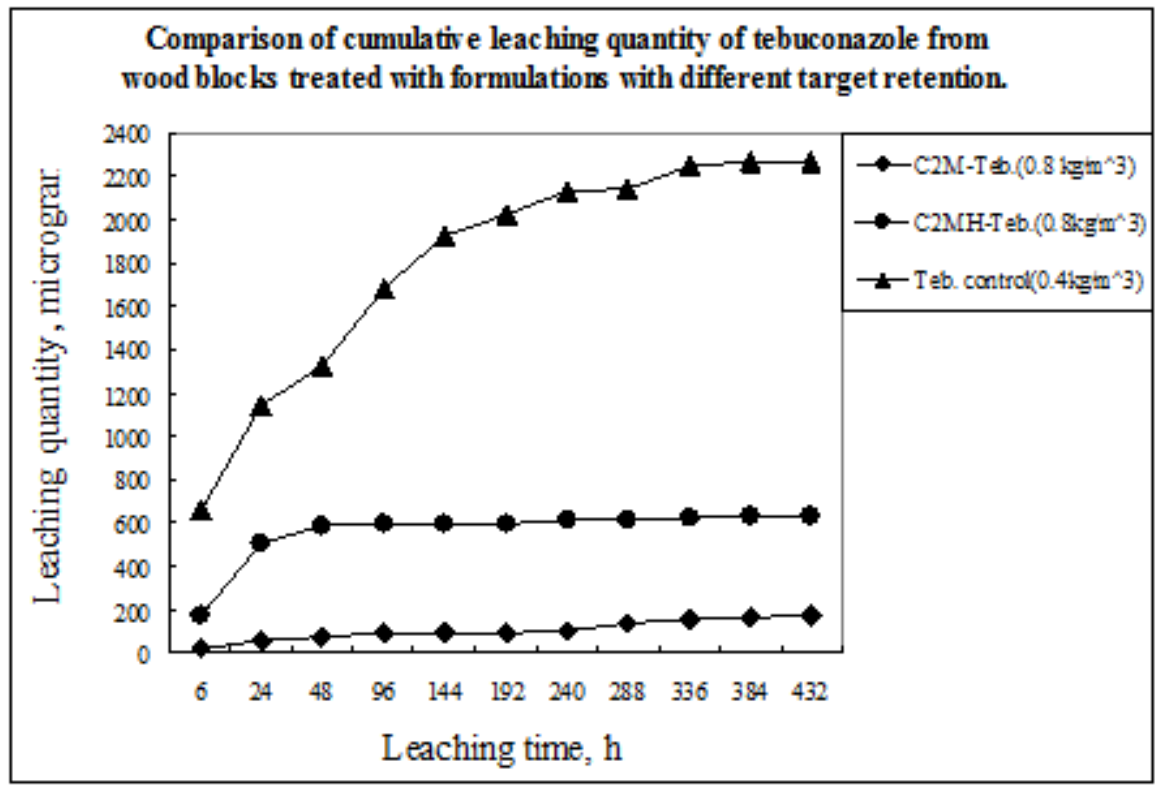

(c)

Figure 4.4. Cumulative leaching quantity of a.i. from wood blocks treated with TEB solution control, C2M-TEB and C2MH-TEB NP formulations.

\subsubsection{Wood Preservation Efficacy By Soil Jar Decay Test}

Soil jar decay tests were performed on already-leached wood blocks treated with C2M-TEB and C2MH-TEB NPs and a solution treated tebuconazole control, according to the AWPA standard method. The decay test results are shown in Figure 4.5. 


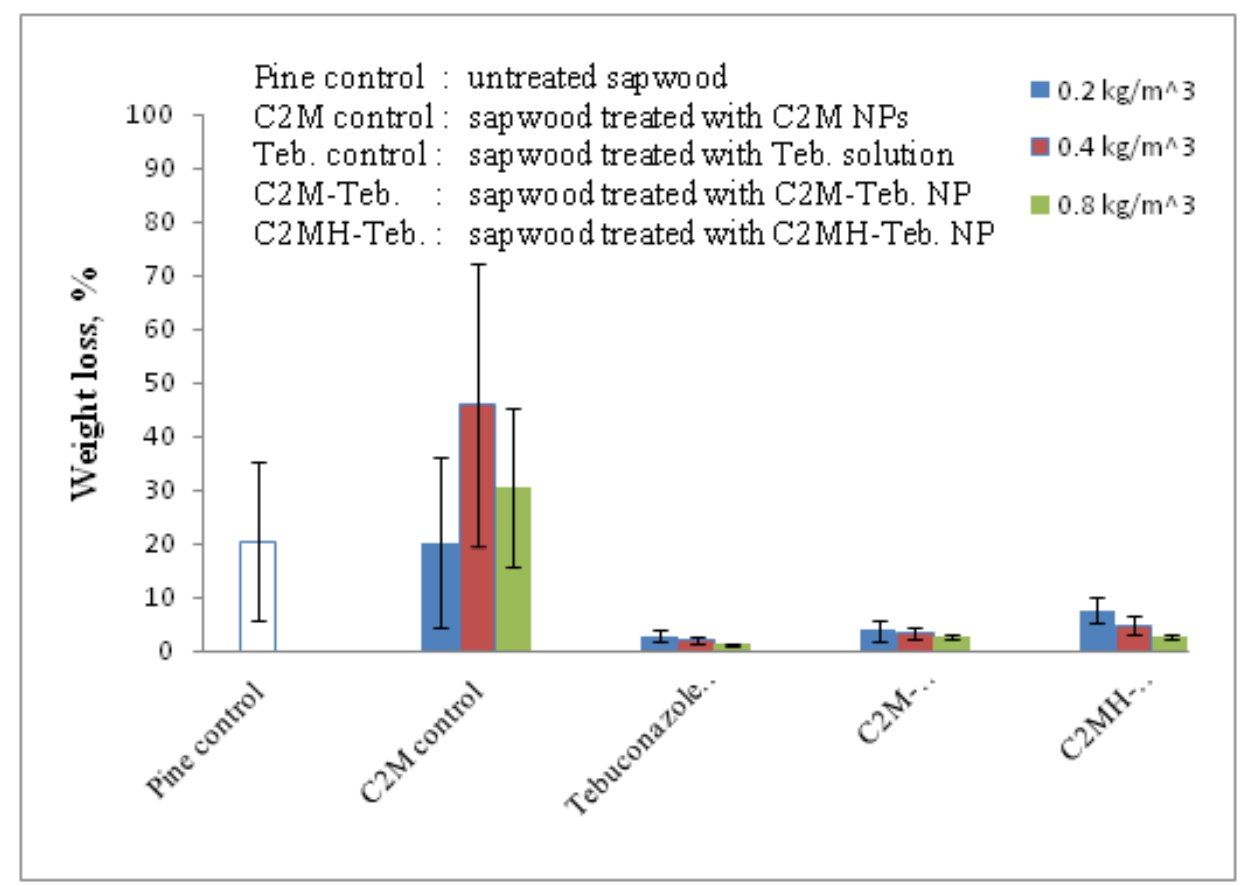

Figure 4.5. Weight loss of leached southern pine sapwood from soil jar decay tests. Theoretical retentions were $0.2,0.4$, and $0.8 \mathrm{~kg}$ tebuconazole $/ \mathrm{m}^{3}$ wood. For $0.2 \mathrm{~kg} / \mathrm{m}^{3}$ wood actual tebuconazole retentions were $0.2,0.13$, and $0.14 \mathrm{~kg} / \mathrm{m}^{3}$ for TEB, C2M-TEB, and $\mathrm{C} 2 \mathrm{MH}-\mathrm{TEB}$ respectively. For $0.4 \mathrm{~kg} / \mathrm{m}^{3}$ wood actual tebuconazole retentions were $0.4,0.24$, and $0.22 \mathrm{~kg} / \mathrm{m}^{3}$ for TEB, C2M-TEB, and C2MH-TEB respectively. For 0.8 $\mathrm{kg} / \mathrm{m}^{3}$ wood actual tebuconazole retentions were $0.795,0.44$, and $0.45 \mathrm{~kg} / \mathrm{m}^{3}$ for TEB, C2M-TEB, and C2MH-TEB respectively.

The weight loss of the $\mathrm{C} 2 \mathrm{M}$ controls (wood treated with $\mathrm{C} 2 \mathrm{M}$ nanoparticles that contain no biocide) may be slightly greater than the untreated pine controls when the nanoparticle "blanks" are introduced at higher levels. If this difference is real than chitosan, like gelatin, may slightly promotes fungal decay by G. trabeum, but the standard deviations are high, so the trend is not definitive. The weight loss for the tebuconazole-containing nanoparticle treated specimens show no significant difference in the preservation efficacy at any retention with any formulation except the C2MH-TEB 
treated wood at a theoretical retention of $0.2 \mathrm{~kg} / \mathrm{m}^{3}$, but with actual retention of 0.14 $\mathrm{kg} / \mathrm{m}^{3}$. Therefore, the tebuconazole introduced in controlled-release NPs retain efficacy against $G$. trabeum, and does so with substantially less leach than tebuconazole introduced in solution.

\subsection{Conclusion}

A simple, efficient, and versatile one-step route was used to prepare self-assembling and biocide-containing core-shell nanoparticles from chitosan grafted with acrylic monomers. The method gives high biocide capture efficiency and a high yield of nanoparticles $(\sim 150 \mathrm{~nm})$ that can be delivered into southern pine sapwood. SEM investigation of nanoparticle-treated field stakes showed nanoparticles penetrated throughout the wood interior. The biocide-containing nanoparticles, C2M-Teb, captured $93 \%$ of the available biocide during their preparation, effectively protected sapwood from biological attack, and showed only $\sim 9 \%$ of the biocide leach as wood blocks treated with a tebuconazole solution. Using this method the nanoparticles' core composition can be manipulated by replacing portions of MMA with other acrylic monomers during the nanoparticle synthesis. This allows the core composition to be appropriately "tuned" for other biocides. When just 2\% of the MMA was replaced with HEMA the nanoparticles possessed a more hydrophilic core, resulting in slightly larger nanoparticles. The wood blocks treated with these nanoparticles leached much more biocide than the $\mathrm{C} 2 \mathrm{M}$ nanoparticle-treated wood, but it was still only $\sim 26 \%$ of the biocide leached by the solution-treated tebuconazole control wood blocks. This shows that very small changes in the core-composition can effectively tune biocide release and minimize biocide leaching. The results demonstrate this method can efficiently incorporate the biocide, significantly reduce biocide leach without compromising wood preservation, and the core-composition is easily altered to allow the nanoparticles to be "tuned" appropriately for other organic biocides. 


\section{Main Findings in This Chapter:}

The biocide-loaded chitosan-g-PMMA nanoparticlesfor low leaching wood preservation were prepared by one-pot synthesis route with biocide capturing efficiency as high as $93 \%$. The biocide leached from treated wood via nanoparticle was only $9 \%$ leached as much quantity as from solution control. The reduced biocide leaching did not compromise wood protection efficacy against biological decay. FESEM analysis confirmed the nanoparticles effectively penetrated throughout wooden stake with dimension of 19x19x445 mm. High biocide capturing efficiency, controllable release rate from nanoparticles, simple and one-pot synthesis route, effective protection efficacy, and cost-effective match the market requirements of using nanotechnology for wood preservation.

\section{Acknowledgements}

The author fully thanks the publisher (Elsevier) for generously offering the author the rights to reuse this published article in my Dissertation without needing further permission.

This work was funded by the United States Environmental Protection Agency Science to Achieve Results (STAR) Program, Grant Number GR832371. The authors thank Dr. Peter Laks, Mr. Glen Larkin, and Mr. Erik Keranen, at MTU School of Forest Resources and Environmental Science, for preparing and pressure treating the wood blocks and Mr. Owen Mills, Department of Materials Science and Engineering at MTU, for the SEM images. 


\section{References:}

1. Salma, U., Chen, N., Richter, D.L.,Filson, P.B., Dawson-Andoh, B., Matuana, L., Heiden, P. (2010),Amphiphilic Core/Shell Nanoparticles to Reduce Biocide Leaching From Treated Wood, 1 - Leaching and Biological Efficacy. Macromolecular Materials and Engineering, 295(5), 442-450.

2. Kakizawa, Y., Nishio, R., Hirano, T., Koshi, Y.,Nukiwa, M.,Koiwa, M., Michizoe, J.,Ida,N. (2010), Controlled release of protein drugs from newly developed amphiphilic polymer-based microparticles composed of nanoparticles. Journal of Controlled Release, 142, 8-13

3. Craparo, E. F., Teresi, G.,Ognibene, M. C., Casaletto, M. P., Bondi', M. L.,Cavallaro, G. (2010), Nanoparticles based on novel amphiphilic polyaspartamide copolymers .J.Nanopart Res, 12, 2629-2644.

4. Kuskov, A. N, Voskresenskaya, A., Goryachaya, A. V., Shtilman, M. I., Spandidos, D. A, Rizos, A. K ,Tsatsakis, A. M. (2010), Amphiphilic poly-N-vinylpyrrolidone nanoparticles as carriers for non-steroidal anti-inflammatory drugs: Characterization and in vitro controlled release of indomethacin.International Journal of Molecular Medicine, 26(1), $85-94$.

5. Kamps, A.C., Sanchez-Gaytan, B.L., Hickey, R.J., Clarke, N.,Fryd, M., \& Park, S.J.(2010), Nanoparticle-Directed Self-Assembly of Amphiphilic Block Copolymers. Langmuir, 26(17), 14345-14350.

6. Leonardis, P.D., Mannina, L., Diociaiuti, M.,Masci, G.(2010), Atom transfer radical polymerization synthesis and association properties of amphiphilic pullulan copolymers grafted with poly(methyl methacrylate). Polymer International, 59(6), $759-765$.

7. Jiang, M., Wu, Y., He, Y., Nie, J.(2010), Synthesis and characterization of an amphiphilic hyperbranched poly(amine-ester)-co-D,L-lactide (HPAE-co-PLA) 
copolymers and their nanoparticles for protein drug delivery. Journal of Applied Polymer Science, 117(2),1156-1167.

8. Aranaz, I., Harris, R.,\&Heras, A.(2010), Chitosan Amphiphilic Derivatives. Chemistry and Applications.Current Organic Chemistry, 14, 308-330.

9. Lao, B., Zhang,Z., Xu, H.H.,Jiang,G. (2010), Novel amphiphilic chitosan derivatives: Synthesis, characterization and micellarsolubilization of rotenone. Carbohydrate Polymers,82,1136-1142.

10. Boehm A.L.,Martinon I., Zerrouk R., Rump E., \&Fessi H.(2003), Nanoprecipitation technique for the encapsulation of agrochemical active ingredients Source: Journal of microencapsulation, 20(4),433 -441.

11. Liu, Y., Laks, P., \&Heiden. P.,(2002), Controlled Release of Biocides in Solid Wood. Part 1. Efficacy against Gloeophyllumtrabeum, a Brown Rot Wood Decay Fungus. $J$. Appl. Polym. Sci ,86, 596.

12. Liu, Y., Laks, P., \&Heiden. P.,(2002), Controlled Release of Biocides in Solid Wood. Part 2. Efficacy against Trametesversicolor and Gloeophyllumtrabeum Wood Decay Fungi J. Appl. Polym. Sci. 86, 608.

13. Liu, Y., Laks, P., \&Heiden. P., (2002), Controlled Release of Biocides in Solid Wood; Part 3. Preparation and Characterization of Surfactant-Free Nanoparticles. J. Appl. Polym. Sci.86, 615.

14. Li,P., Zhu,J., Sunintaboon,P., \&Harris, F.W.(2002),New Route to Amphiphilic Core-Shell Polymer Nanospheres: Graft Copolymerization of Methyl Methacrylate from Water-Soluble Polymer Chains Containing Amino Groups. Langmuir,18 (22), $8641-8646$.

15. Qian,F., Cui, F., Ding, J., Tang, C., \& Yin, C.(2006), Chitosan Graft Copolymer Nanoparticles for Oral Protein Drug Delivery: Preparation and Characterization, Biomacromolecules, 7, 2722-2727. 
16. American Wood-Preservers Association Book of Standards, 1998. Standard Method of Testing Wood Preservatives by Laboratory Soil-Block Culture E10-91. Branbury, Texas, E10-91. 375-385.

17. Kreuter. J. (1978), Nanoparticles and Nanocapsules-New Dosage Forms in the Nanometer Size Range, Pharm ActaHelv. 53(2). 
Comparing Leaching of Different Copper Oxide Nanoparticles and Ammoniacal Copper Salt from Wood

Xiaochu Ding ${ }^{\text {a }}$, Martha Barajas Meneses ${ }^{a}$, Soha M. Albukharia, Dana L. Richter ${ }^{b}$, Laurent M. Matuana ${ }^{\mathrm{c}}$, Patricia A. Heiden ${ }^{\mathrm{a}, *}$

a Department of Chemistry, Michigan Technological University, Houghton, MI 49931, USA

b School of Forest Resources and Environmental Science, Michigan Technological University, Houghton, MI 49931, USA c School of Packaging, Michigan State University, East Lansing, MI 48824, USA

The initial idea for this work was conceived by Dr. Heiden. All the experiments in this chapter were design and conducted by Mr. Xiaochu Ding except the following: the Soil Jar Decay test which was conducted by Dr. Dana Richter in his lab, and parts of leaching test were conducted by Soha M. Albukhari and Martha Barajas Meneses, who were Ph.D. students in the same research group at the time this work was done, and Dr. Laurent Matuana who analyzed copper content in the treated wood.

Mr. Xiaochu Ding

Department of Chemistry

Michigan Technological University

Ms. Soha M. Albukhari

Department of Chemistry

Michigan Technological University

Dr. Laurent M. Matuana

School of Packaging

Michigan State University
Dr. Martha Barajas Meneses

Department of Chemistry

Michigan Technological University

Dr. Dana L. Richter

School of Forest Resources and Environmental Science

Michigan Technological University

Dr. Patricia A. Heiden*

Department of Chemistry

Michigan Technological University 


\section{Chapter 5}

Comparing Leaching of Different Copper Oxide Nanoparticles and Ammoniacal Copper Salt from Wood

Xiaochu Ding a , Martha Barajas Meneses ${ }^{a}$, Soha M. Albukhari ${ }^{\mathrm{a}}$, Dana L. Richter ${ }^{\mathrm{b}}$, Laurent M. Matuana ${ }^{\mathrm{c}}$, Patricia A. Heiden ${ }^{\mathrm{a}, *}$

a Department of Chemistry, Michigan Technological University, Houghton, MI 49931, USA

b School of Forest Resources and Environmental Science, Michigan Technological University, Houghton, MI 49931, USA

c School of Packaging, Michigan State University, East Lansing, MI 48824, USA

*Corresponding author. Tel.: +1 906487 3452; Fax: +1 9064872061.

E-mail address: paheiden@mtu.edu (Patricia A. Heiden)

This research was funded by the United States Environmental Protection Agency Science to Achieve Results (STAR) Program, Grant Number GR832371.

This chapter is in press in the journal Macromolecular Materials and Engineering, 2013, DOI: 10.1002/mame.201200439. 


\begin{abstract}
Copper-based nanoparticle wood preservatives typically contain a mixture of nanoparticle sizes. We wanted to know if nanoparticle size was a significant effect in quantity of leach and if the leachate contained nanoparticles. To do this we prepared copper oxide nanoparticles with uniform diameters of $10 \mathrm{~nm}$ and $50 \mathrm{~nm}$, and measured the leachate from southern pine sapwood treated with these nanoparticles. We also studied the effect of polymer stabilizers on nanoparticle leaching. It was found that significant numbers of nanoparticles leached from the treated wood. The trends showed that nanoparticle size was a major factor in leaching. The $50 \mathrm{~nm}$ nanoparticles leached slightly less than a soluble copper salt control, but $10 \mathrm{~nm}$ nanoparticles leached substantially more than the control. Polymer stabilizers increased leaching. The cumulative leaching, calculated as $\%$ loss of initial $\mathrm{Cu}^{2+}$ retention, was $15.6 \%, 14.3 \%$, and $12.3 \% \mathrm{Cu}^{2+}$ for $10 \mathrm{~nm}$ nanoparticles stabilized with a slightly acidic stabilizer, a slightly basic stabilizer, and unstabilized $10 \mathrm{~nm}$ nanoparticles, versus only $6.7 \%$ for the quaternary ammonium control. An effort to determine if $10 \mathrm{~nm}$ nanoparticles had greater efficacy against gloeophyllum trabeum brown rot fungus failed due to unexpectedly low retention levels. These findings indicate that nanoparticle size is a major factor in leaching and that nanoparticles themselves can leach from treated wood.
\end{abstract}

Keywords: biocide, copper oxide, leaching, nanoparticle, wood preservation 


\subsection{Introduction}

Solid wood intended for exterior use must be protected from biological decay by treatment with wood preservatives (1). Copper chromated arsenic (CCA) was long the dominant preservative used to treat wood for home applications, but as CCA was being phased out for use around the home, alternative forms of copper were developed. These newer copper-based preservatives, including the so-called "micronized" copper, were recently reviewed (2).

Soluble forms of copper, such as the ammoniacal copper systems (ACQ, A, B, C, and D), copper amine systems and copper azole systems, as well as water-borne and oil-borne copper naphthalene systems, are now typically used. However, "micronized" copper particle systems are making significant inroads in the wood preservative industry. These solid copper salts may be delivered in aqueous suspensions that may include other biocide components, along with unspecified polymeric dispersants (2). The term "micronized" suggests larger particles, but these commercial systems appear to be much smaller with many particles less than $100 \mathrm{~nm}$ in diameter (3) so they should more properly be termed "nanocopper" systems. For example, Osmose's copper carbonate formulations are thought to be comprised of nanoparticles with an average diameter of approximately 80,120 , and $250 \mathrm{~nm}(4)$. A formulation with an average diameter of approximately $40 \mathrm{~nm}$ for "refractory" wood species also exists (5).

Use of bioactive nanoparticles has been debated both for concerns about the environmental implications and some disagreement about "threshold levels" (minimal level required for biological effect) of copper-based nanoparticles. Also, questions remain about if these nanoparticles are more effective for protection against fungal attack than soluble systems. Copper-based nanoparticles are often claimed to have higher efficacy and lower leach than soluble forms of copper wood preservatives. However, the published literature on "nanocopper" is somewhat limited and not always in agreement. 
Different commercial systems tested against Gloeophyllum trabeum report threshold values as low as $0.6 \mathrm{~kg} \mathrm{CuO} / \mathrm{m}^{3}$ wood to as high as $4.2 \mathrm{~kg} \mathrm{CuO} / \mathrm{m}^{3}$ wood. Some research has shown that these copper systems have greater efficacy than traditional soluble copper systems, while other research notes that greater efficacy is not observed against all species (2). For example, Clausen and co-workers found that copper-based nanoparticles (from Nanoarc) were more effective in soil jar tests than soluble copper salts against $G$. trabeum and T. versicolor, but had only similar or less effect when tested against Antrodia sp., various species of mold, and termites (Reticulitermes flavipes kollar) (6). Some reports using Formosan termites showed similar efficacy between nanoparticle and soluble copper systems (2). Environmental concerns with nanoparticle copper systems stem largely from uncertainties about the environmental fate of the nanoparticles $(\mathbf{4 , 7 - 9})$ and awareness of the toxicity of copper oxide nanoparticles to non-target species (10). Studies that address biocide leaching from wood treated with nanoparticle copper systems appear to be largely confined to the patent literature (11), although published studies do address the fate of nanoparticles under various conditions. For example, two recent studies described the fate of copper oxide nanoparticles in soils $(\mathbf{1 2 , 1 3 )}$. Those researchers found that the nanoparticles tended to aggregate, but many factors influenced their mobility in soils, including dissolved organic matter and ionic strength. Another recent publication addressed the fundamental issues affecting the aggregation and deposition of engineered nanomaterial in aquatic environments (14). Benn and Westerhoff also showed that Ag nanoparticles could leach from socks during washing, and that significant differences in leaching occurred based on the type of sock tested, which suggested that the manufacturing process strongly influenced nanoparticle leaching (15).

As stated above, toxicity of nanoparticles against different species is also in question. For example, antimicrobial activity of different nanoparticles, including $\mathrm{CuO}$, was found 
against the beneficial soil microbe Pseudomonas putida KT2440. Nano-CuO and solvated copper ions both had toxicity against this microbe, but the bulk form (micro meters to macro size) of $\mathrm{CuO}$ was non-toxic (16). The authors also cited a report that claimed that $\mathrm{ZnO}$ nanoparticles of $8 \mathrm{~nm}$ were more toxic to $S$. aureus than nanoparticles of 50-70 nm (17).

Outside of the patent literature and abstracts from professional meetings little research on leaching of $\mathrm{CuO}$ nanoparticles is published. The most detailed information found was a review by Freeman and McIntyre (2)that referenced professional meetings and workshops, and more recently by Kartal and co-workers (6). These works described results from commercial systems with proprietary compositions that were not always specific about nanoparticle size. It was also unclear if these formulated systems contained additives that had biological activity or that may have enhanced adhesion to wood, i.e. "fixation". If fixation was promoted in these systems the mechanism was likely to be based on approaches similar to those in conventional copper systems. Those systems often use amines that promote adhesion through chemical interactions with acid and phenolic groups of lignin, or use ethanolamine to form a 5-member ring complex with solubilized copper ions that also preferentially interact with lignin $(\mathbf{2 , 1 8})$. We are not aware of reports of nanoparticles themselves being leached from treated wood, but if this occurs, it seems that there is a probability of leaching being affected not only by the treating composition but also wood species and treating conditions.

Answering all these questions is beyond the scope of this paper. Our objective here is to answer the following basic questions regarding copper-based nanoparticle preservatives: does nanoparticle-treated wood leach copper as nanoparticles; if nanoparticles are leached is nanoparticle size a factor; do polymer stabilizers affect the amount of copper leached; and can extremely small $\mathrm{CuO}$ nanoparticles give biological efficacy against fungal attack at extremely low levels of treatment? 
To answer these questions we treated pine sapwood with $\mathrm{CuO}$ nanoparticles, synthesized using procedures known to give nanoparticles with uniform size (19), and a soluble copper salt (ACQ) as a control. We collected and measured leachate by standardized methods and used Transmission Electron Microscopy (TEM) to both verify the uniformity of nanoparticle size initially, and to check the leachate for the presence of $\mathrm{CuO}$ nanoparticles. The effect of nanoparticle size on leaching was studied using 10 and 50 $\mathrm{nm} \mathrm{CuO}$ nanoparticles. To study the effects of polymer additives on leaching we stabilized some $\mathrm{CuO}$ nanoparticles with a slightly basic polyvinylpyrrolidone (PVP) and others with a slightly acidic modified chitosan. To determine if the nanoparticles have biological effect at low "loading" levels of $\mathrm{CuO}$ nanoparticles in the treated pine sap wood at levels below the typical minimal treatment levels for copper which is often taken as $2 \mathrm{~kg} \mathrm{Cu}^{2+} / \mathrm{m}^{3}$ of wood. 


\subsection{Experimental Section}

\subsubsection{Materials}

Poly(vinylpyrrolidone) $\left(\mathrm{M}_{\mathrm{w}} \sim 24,000 \mathrm{~g} / \mathrm{mol}\right)$, glycidyltrimethylammonium chloride (GTMAC), sodium hydroxide (pellets, 97+\%), copper (II) acetate monohydrate ( 98+\%) and acetic acid (99.7+ \%) were purchased from Sigma-Aldrich. Chitosan (70\% deacetylation) was donated by Cochin University of Science and Technology (Cochin, India). Copper standard solution ( $1 \mathrm{mg} \mathrm{Cu} \mathrm{Cu}^{2+} / \mathrm{mL}$ in $2 \% \mathrm{HNO}_{3}$ aqueous solution), $\mathrm{H}_{2} \mathrm{SO}_{4}$ conc. (98.0\%), and hydrogen peroxide (30\% aqueous solution) were purchased from ACROS ORGANICS, VWR, and Mallinckrodt Chemicals, respectively. All reagents were used as received. ACQ solution $\left(15 \mathrm{mg} \mathrm{Cu}^{2+} / \mathrm{mL}\right)$ and wood blocks cut to dimensions of $19 \mathrm{~mm}$ x $19 \mathrm{~mm}$ x $19 \mathrm{~mm}$ from southern pine sapwood were donated by MTU School of Forest Resources and Environmental Science, and was used as received. Fungal tests used Gloeophyllum trabeum (ATCC 11539), a basidiomycete brown rot wood decay fungus.

\subsubsection{Preparation of Water-Soluble Modified Chitosan (HTCC)}

A water-soluble chitosan derivative, [(2-hydroxy-3-trimethylammonium)propyl] chitosan chloride (HTCC) was synthesized according to the reported procedure (20), where chitosan (3.0 g, 70\% deacetylated) was dispersed in $30 \mathrm{~mL}$ of deionized (D.I.) water at $85^{\circ} \mathrm{C}$ in a round bottom flask equipped with condenser and magnetic stirring bar. GTMAC (10.7 $\mathrm{mL}, 55.5 \mathrm{mmol})$ was then added to the chitosan suspension in 3 portions $(3.6 \mathrm{~mL} \times 3)$ at $2 \mathrm{~h}$ intervals. The reaction was maintained for $24 \mathrm{~h}$ at $85{ }^{\circ} \mathrm{C}$ with vigorous magnetic stirring. The resulting yellowish solution was diluted with $50 \mathrm{~mL}$ of D.I. water and allowed to stand for $6 \mathrm{~h}$. The product was collected from the upper layer by precipitating in cold acetone. The product was re-dissolved in D.I. water and precipitated two additional times to purify it. The solid product was dried under vacuum 
at $50{ }^{\circ} \mathrm{C}$ for $12 \mathrm{~h}$ to yield $7.57 \mathrm{~g}$ of water-soluble chitosan derivative. Scheme 5.1 shows the chemical reaction.

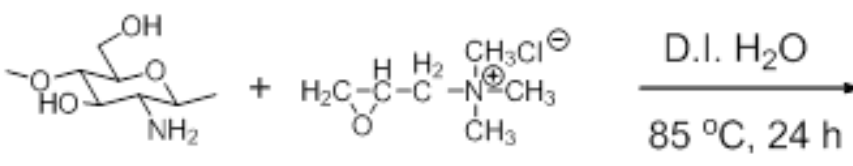

Chitosan
GTMAC

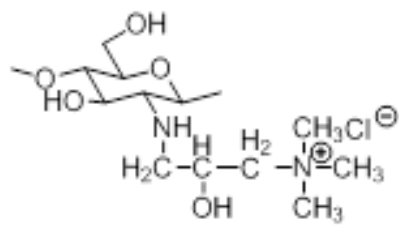

HTCC

Scheme 5.1. Synthesis of [(2-hydroxy-3-trimethylammonium) propyl] chitosan (HTCC), a water-soluble chitosan derivative.

\subsubsection{Preparation of Polymer-Stabilized and Unstabilized CuO Nanoparticles}

$\mathrm{CuO}$ nanoparticles $(\sim 10 \mathrm{~nm})$ were prepared using a modification of reported methods $(\mathbf{1 9 , 2 1 , 2 2})$. Glacial acetic acid $(1.0 \mathrm{~mL}, 17.5 \mathrm{mmol})$ was added to aqueous copper acetate $(300 \mathrm{~mL}, 0.02 \mathrm{M})$ at room temperature. The temperature was increased to $100{ }^{\circ} \mathrm{C}$, and then finely ground sodium hydroxide $(1.0 \mathrm{~g}, 25 \mathrm{mmol})$ was quickly added with vigorous magnetic stirring. The reaction was continued for $6 \mathrm{~h}$ to yield a dark suspension. The $\mathrm{CuO}$ nanoparticles were collected by centrifugation at $4000 \mathrm{rpm}$, washed with D.I. water (3 x $10 \mathrm{~mL}$ ) to remove the unreacted chemicals, and re-dispersed in D.I. water by sonication. $\mathrm{CuO}$ nanoparticles with $\sim 10 \mathrm{~nm}$ diameter were produced as long as the concentration of the copper acetate solution was $0.02-0.12$ and the temperature and molar ratio of copper acetate : acetic acid : sodium hydroxide were constant. The purified $\mathrm{CuO}$ nanoparticle suspension was diluted with D.I. water to $100 \mu \mathrm{g} / \mathrm{mL}$ for TEM analysis (JEOL JEM-4000FX, Tokyo, Japan) and showed a uniform size of $\sim 10 \mathrm{~nm}$. CuO nanorods with $\sim 50 \mathrm{~nm}$ diameter were prepared by changing the feedstock ratio and temperature. At $50{ }^{\circ} \mathrm{C}, 2.5 \mathrm{~g}(62.5 \mathrm{mmol})$ of sodium hydroxide was added to $300 \mathrm{~mL}$ of $0.1 \mathrm{M}$ aqueous solution $(30 \mathrm{mmol})$ of copper acetate mixed with $2.5 \mathrm{~mL}$ (40 mmol) of glacial acetic acid. The reaction mixture was maintained at $50{ }^{\circ} \mathrm{C}$ for $30 \mathrm{~min}$ and then increased to $\sim 120{ }^{\circ} \mathrm{C}$ for $5 \mathrm{~h}$. The as-made suspension was cooled down to room 
temperature, and the $\mathrm{CuO}$ nanoparticles were collected by centrifugation, washed with D.I. water (3 x $10 \mathrm{~mL})$ and centrifuged at $4000 \mathrm{rpm}$ to remove unreacted chemical and isolate small size nanoparticles, and re-dispersed in D.I. water. The purified $\mathrm{CuO}$ nanorod suspension was similarly diluted to $100 \mu \mathrm{g} / \mathrm{mL}$ for TEM analysis, and showed nanorods with $\sim 50 \mathrm{~nm}$ length and $\sim 10 \mathrm{~nm}$ diameter.

Polymer-stabilized $\mathrm{CuO}-10$ nanoparticles were prepared the same way as the unstabilized nanoparticles above except in the presence of HTCC or PVP as a stabilizer. For example, HTCC (0.96 g, theoretical wt. ratio of 1:1 HTCC:CuO) was dissolved in a $0.04 \mathrm{M}$ aqueous solution of copper acetate (12 mmol in $300 \mathrm{~mL}$ D.I. water) mixed with glacial acetic acid $(2.0 \mathrm{~mL}, 35 \mathrm{mmol})$ in a $500 \mathrm{~mL}$ round bottom flask. The mixture was vigorously stirred for $1 \mathrm{~h}$ at $100{ }^{\circ} \mathrm{C}$ to give a clear blue solution. Then finely ground $\mathrm{NaOH}$ pellets $(2.0 \mathrm{~g}, 50 \mathrm{mmol})$ were quickly added and the reaction was kept for $6 \mathrm{~h}$ at $100^{\circ} \mathrm{C}$ with vigorous magnetic stirring. After cooling down to room temperature the HTCC-stabilized $\mathrm{CuO}$ nanoparticles were similarly collected by centrifugation, washed with D.I. water $(3 \times 10 \mathrm{~mL})$, and then re-dispersed in D.I. water by sonication to form a stable HTCC-stabilized $\mathrm{CuO}$ nanoparticle suspension. TEM micrographs showed a uniform particle size of $\sim 10 \mathrm{~nm}$.

HTCC-stabilized CuO-50 nanoparticles were prepared in the same way as CuO-50 except in the presence of HTCC. Particle size by TEM was $\sim 50 \mathrm{~nm}$.

The nanoparticles are designated as follows: $\mathrm{CuO}$ nanoparticles without polymer stabilizing are designated $\mathrm{CuO}-10$ and $\mathrm{CuO}-50$ for the $10 \mathrm{~nm}$ and $50 \mathrm{~nm}$ nanoparticles respectively, while $\mathrm{CuO}$ nanoparticles with a PVP polymer stabilizer are PVP-CuO-10, and HTCC stabilized nanoparticles are HTTC-CuO-10 or HTTC-CuO-50, depending on the nanoparticle size. The synthesis is illustrated in Scheme 5.2. 


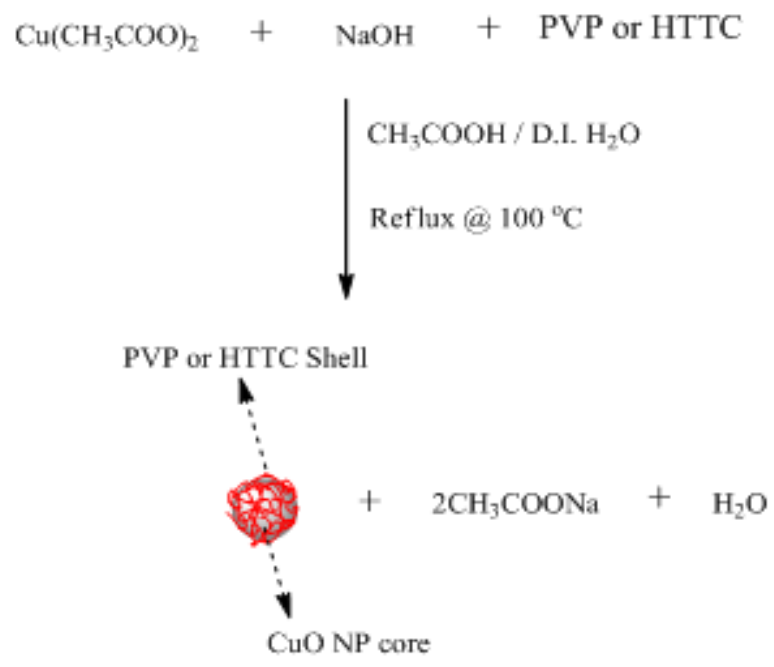

Scheme 5.2. Synthesis of polymer-stabilized $\mathrm{CuO}$ nanoparticles by aqueous thermal hydrolysis using D.I. water as the solvent and heating at reflux.

\subsubsection{Preparation of ACQ}

ACQ was prepared for us in the Department of Forest Resources and Environmental Science using the following standard procedure. Copper carbonate (equivalent to $68.75 \%$ $\mathrm{CuO}, 931.4 \mathrm{~g}$ ), didecyldimethylammonium chloride (DDAC, 80\% solution, $399.6 \mathrm{~g}$ ), and ethanolamine $(100 \%, 1760 \mathrm{~g})$ were added to water $(908.1 \mathrm{~g})$ and stirred to give a solution that is $24 \%$ in the active ingredient. The prepared ACQ solution was then diluted with D.I. water to a solution with a concentration of $15 \mathrm{mg} \mathrm{Cu}^{2+} / \mathrm{mL}$.

\subsubsection{Measurement of $\mathrm{Cu}^{2+}$ Concentration}

The $\mathrm{Cu}^{2+}$ concentration in the suspensions and ACQ solution was measured by atomic absorption spectrophotometry (BUCK Scientific Model 200A, BUCK Scientific Inc, USA). Briefly, $2.0 \mathrm{~mL}$ of as-made nanoparticle suspensions or ACQ solution was transferred to a beaker and heated to dryness, followed by adding concentrated $\mathrm{H}_{2} \mathrm{SO}_{4}(3$ $\mathrm{mL})$ and $\mathrm{H}_{2} \mathrm{O}_{2}(2 \mathrm{~mL}, 30 \%$ solution) to digest under gentle boiling until a clear light blue solution was obtained. The digested sample was transferred to a $250 \mathrm{~mL}$ volumetric flask and D.I. water was added to scale. A series of copper standard solutions was prepared to 
give the calibration curve shown in Figure 5.1. The concentration of $\mathrm{Cu}^{2+}$ was calculated using Beer's law and found to be $4.6 \mathrm{mg} \mathrm{Cu}^{2+} / \mathrm{mL}$ (CuO-10 control), $4.8 \mathrm{mg} \mathrm{Cu}^{2+} / \mathrm{mL}$ (PVP-CuO-10), $3.8 \mathrm{mg} \mathrm{Cu}{ }^{2+} / \mathrm{mL}$ (HTTC-CuO-10), $5.6 \mathrm{mg} \mathrm{Cu}^{2+} / \mathrm{mL}$ (CuO-50 control), $6.8 \mathrm{mg} \mathrm{Cu}^{2+} / \mathrm{mL}$ (HTCC-CuO-50) and $15 \mathrm{mg} \mathrm{Cu}^{2+} / \mathrm{mL}$ (ACQ solution).

\subsubsection{Treatment of Wood Blocks}

Wood blocks (19x19x19 mm) were extracted in warm alcohol for $24 \mathrm{~h}$ to remove soluble extractives and wood sawdust near the surface pores and then dried at $40{ }^{\circ} \mathrm{C}$ for $48 \mathrm{~h}$ in order to achieve maximum delivery of $\mathrm{CuO}$ nanoparticles. The pre-treated wood blocks were then treated with a nanoparticle suspension or ACQ solution according to the standard method of Wood Pressure Treatment (AWPA E11-97). The quantity of nanoparticle suspension needed to deliver target retentions of $0.64,2.0$, and $4.0 \mathrm{~kg} \mathrm{Cu}^{2+}$ / $\mathrm{m}^{3}$ wood was measured and the suspension was then diluted to $90 \mathrm{~mL}$ by D.I. water. The volume of $90 \mathrm{~mL}$ was required to sufficiently cover 6 wood blocks having dimensions of $19 \mathrm{~mm}$ x $19 \mathrm{~mm}$ x $19 \mathrm{~mm}$ when these blocks were placed in a beaker. The wood blocks were covered with a plastic mesh and aluminum blocks to keep them submerged throughout the pressure-treatment process. The beaker was transferred into the pressure cylinder and subjected to a pressure treatment consisting of a partial vacuum of less than $25 \mathrm{~mm} \mathrm{Hg}$ for $30 \mathrm{~min}$, followed by pressurization to $100 \mathrm{psi}$ for $1 \mathrm{~h}$. The specimens were removed from cylinder, and dried at $40{ }^{\circ} \mathrm{C}$ for $48 \mathrm{~h}$ and then dried at air atmosphere under room temperature for $24 \mathrm{~h}$. The mass of each dry block was weighed, and the density of each treated dry block was calculated according to equation (1):

$$
\rho_{\text {Block }}=\frac{M_{\text {Block }}}{V_{\text {Block }}}
$$

Where the volume for each block was: $V_{\text {Block }}=19 \times 19 \times 19\left(\mathrm{~mm}^{3}\right)=6859\left(\mathrm{~mm}^{3}\right)$.

Wood blocks treated for desired target retentions were digested to determine the actual copper retention. The wood blocks were first ground into powder, and a known 
quantity of that powder was weighed out and identified as $M_{\text {Sample }}$. Each sample was digested by adding the appropriate amount of $\mathrm{H}_{2} \mathrm{SO}_{4}$ (conc.) and hydrogen peroxide (30\% aqueous solution). Then the samples were heated until all solids were digested to form a clear solution, then transferred into a volumetric flask, and diluted to scale using D.I. water. Previously prepared standard copper solutions were analyzed by atomic absorption spectrophotometer to give a calibration curve. Then sample solutions were analyzed to measure the concentration of copper ion $\left(\mathrm{C}_{\mathrm{Cu}}{ }^{2+}\right)$ and the actual concentration of copper was calculated according to the calibration curve and Beer's law. The actual retention of $\mathrm{Cu}^{2+}$ in each block was calculated by equation (2):

$$
\text { Actual Retention }=\frac{C_{\mathrm{Cu}^{2+}} \times V_{\mathrm{Cu}^{2+}} \times \rho_{B l o c}}{M_{S \text { a mple }}}
$$

A wooden stake with dimensions of $19 \mathrm{~mm}$ x $19 \mathrm{~mm}$ x $455 \mathrm{~mm}$ was also treated with PVP-stabilized $\mathrm{CuO}$ nanoparticles. A central section was cut out and FESEM analysis (Hitachi S-4700, Hitachi High Technologies America, Inc.) was performed to confirm effective penetration of nanoparticles throughout the interior of the treated stakes.

\subsubsection{Leaching Tests}

Leaching tests were performed according to The American Wood Preservers Association Standards (AWPA E11-97) (23). Leachate was collected in a beaker and heated at $80^{\circ} \mathrm{C}$ to remove water. $\mathrm{H}_{2} \mathrm{SO}_{4 \text { (conc.) }}(\sim 4 \mathrm{~mL})$ and $\mathrm{H}_{2} \mathrm{O}_{2}(2-4 \mathrm{~mL}, 30 \%$ solution) were added to digest the solids (the digestion reagent quantities were determined according to the amount of leachate). The beaker was covered with a watch glass and heated to boiling until the digestion was completed and a clear solution formed. The digestion dissolves solid nanoparticles so $\mathrm{Cu}^{2+}$ ions can be detected. Due to the small quantity and small size of the nanoparticles we did not attempt to separate $\mathrm{CuO}$

nanoparticles to quantify the relative numbers of nanoparticles versus $\mathrm{Cu}^{2+}$ ions in the 
leachate because the relative error would be too high.

The digested solution was transferred into a volumetric flask $(250.00 \mathrm{~mL}$ volumetric flask for high concentration leachate, $50.00 \mathrm{~mL}$ or $25.00 \mathrm{~mL}$ volumetric flask for low concentration leachate), and D.I. water was added to the scale. A series of $\mathrm{Cu}^{2+}$ standard solutions was prepared for calibration curves by atomic absorption analysis (Figure 5.1). By this method, leachate was collected at regular leaching intervals, and the concentration (X value) of $\mathrm{Cu}^{2+}$ in the leachate was measured by its absorbance (Y value) on the calibration curve regression equation, so the quantity of the $\mathrm{Cu}^{2+}$ of each leachate was obtained by equation (3).

$$
\text { Amount }_{\mathrm{Cu}^{2+}}=X \times V_{\text {Sample solution }}
$$

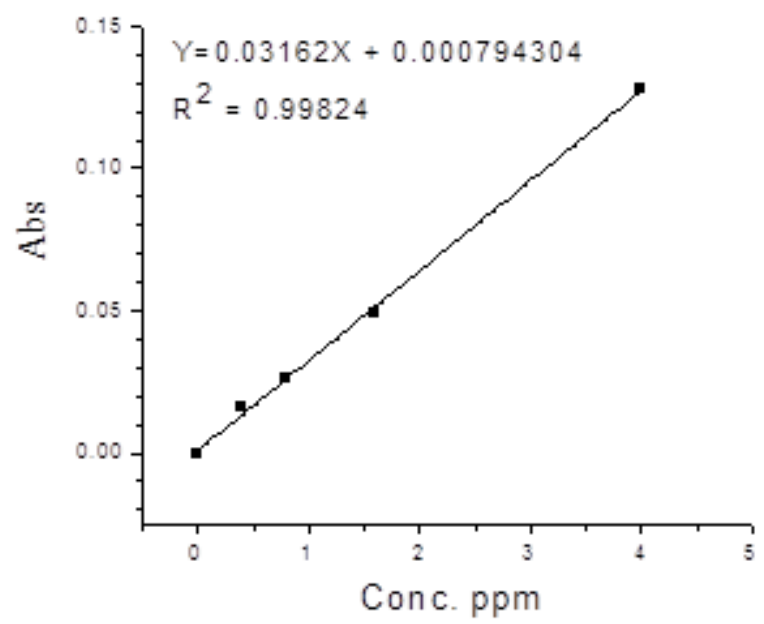

Figure 5.1. A standard curve for analysis of copper in nanoparticle suspensions or leachate by atomic absorption spectrophotometry.

\subsubsection{Soil Jar Decay Test}

Treated wood blocks (southern pine sapwood, $19 \mathrm{~mm} \times 19 \mathrm{~mm} \times 19 \mathrm{~mm}$ ) were dried at $40{ }^{\circ} \mathrm{C}$ for $24 \mathrm{~h}$. Another batch of wood blocks were leached in water for three weeks, and then dried at $40{ }^{\circ} \mathrm{C}$ for $24 \mathrm{~h}$. The mass of the dried wood blocks was measured to $\pm 0.0005 \mathrm{~g}$. 
These two batches were designated as "unleached" and "leached" specimens. All blocks were exposed to the brown rot fungus, Gloeophyllum trabeum ATCC 11539 for 12 weeks using American Wood Preservers Association (AWPA) testing method E-10-07, "Standard Method of Testing Wood Preservatives by Laboratory Soil Block Cultures”. 


\subsection{Results and Discussion}

\subsubsection{Preparation and Characterization of Polymer-Stabilized CuO Nanoparticles}

Both alco-thermal (21) and aqueous-thermal (19) methods were initially tested as routes to give $\mathrm{CuO}$ nanoparticles. The aqueous-thermal method was selected to use here because it gave nanoparticles with a uniform size that were relatively stable even without a polymer stabilizer. $\mathrm{CuO}$ nanoparticles of $\sim 10 \mathrm{~nm}$ are designated as $\mathrm{CuO}-10$, and when prepared in the presence of either PVP or HTCC polymer-stabilized nanoparticles are obtained that are designated as PVP-CuO-10 and HTTC-CuO-10, respectively (Scheme 5.2).

The aqueous-thermal method is reported to give $10 \mathrm{~nm}$ nanoparticles when the copper acetate concentration was $0.02 \mathrm{M}$. We obtained spherical $10 \mathrm{~nm}$ diameter nanoparticles as long as the copper acetate concentration in the aqueous solution was kept between $0.02-0.12 \mathrm{M}$, and the ratio of copper acetate : acetic acid : sodium hydroxide was kept constant. When polymer stabilizer was used the ratio of PVP or HTCC to CuO was maintained at 1:1 w/w. The size and shape of the nanoparticles prepared under these conditions was verified by TEM (Fig.5.2a, b).Suspensions of PVP-stabilized and HTCC-stabilized $\mathrm{CuO}$ nanoparticles appeared to be stable for several months at a concentration of $3.8-4.8 \mathrm{~g} \mathrm{CuO} / \mathrm{L}$.

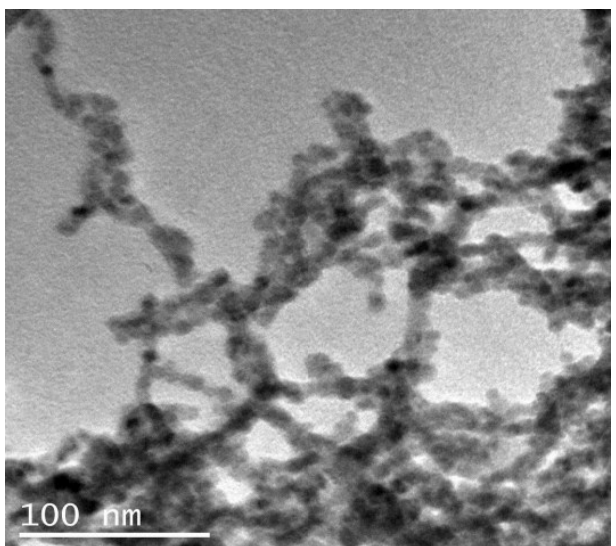

(a)

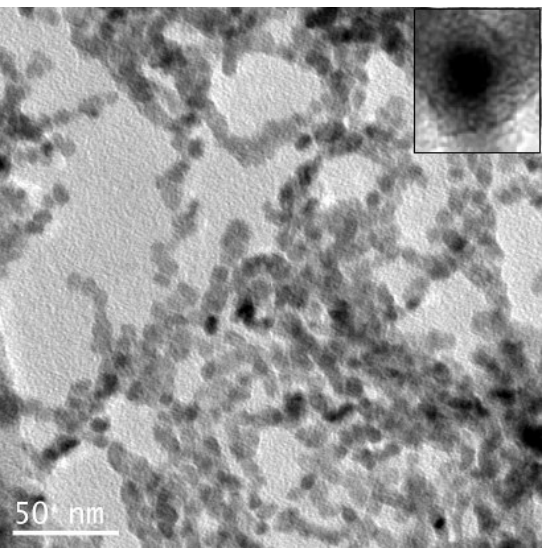

(b)

Figure 5.2. Representative TEM images of (a) unstabilized CuO-10 nanoparticles and (b) 166 
PVP-stabilized CuO-10 nanoparticles, prepared by aqueous-thermal hydrolysis.

Based on these studies the only variable that had a significant effect on the final size of the $\mathrm{CuO}$ nanoparticles was the concentration of the initial copper salt and the reaction temperature. $\mathrm{CuO}$ nano-rods with a length of $\sim 50 \mathrm{~nm}$ and a diameter of $\sim 10 \mathrm{~nm}$ were made by adding sodium hydroxide at $50{ }^{\circ} \mathrm{C}$ and heating at $120{ }^{\circ} \mathrm{C}$ using copper acetate concentration at $0.1 \mathrm{M}$. In the presence of $\mathrm{HTCC}$ or PVP a stable polymer-stabilized $\mathrm{CuO}$ nano-rod was prepared. TEM images of CuO-50 and HTCC-CuO-50 nano-rods are shown in Figure 5.3 a,b respectively.

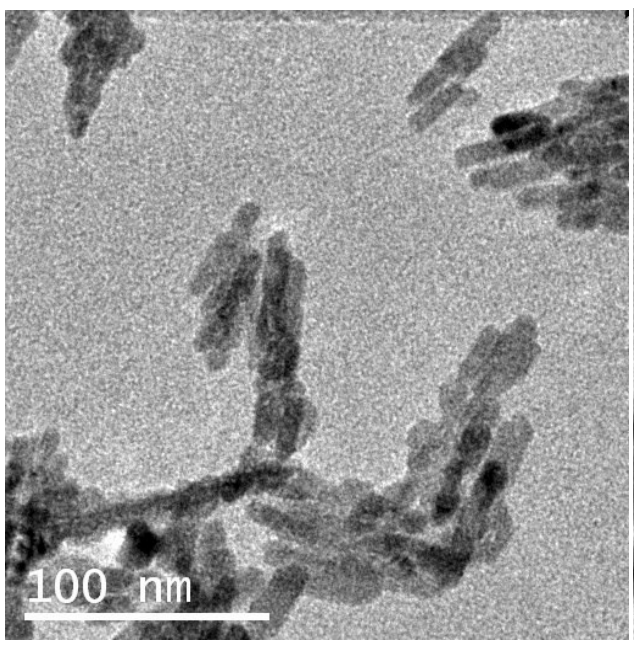

(a)

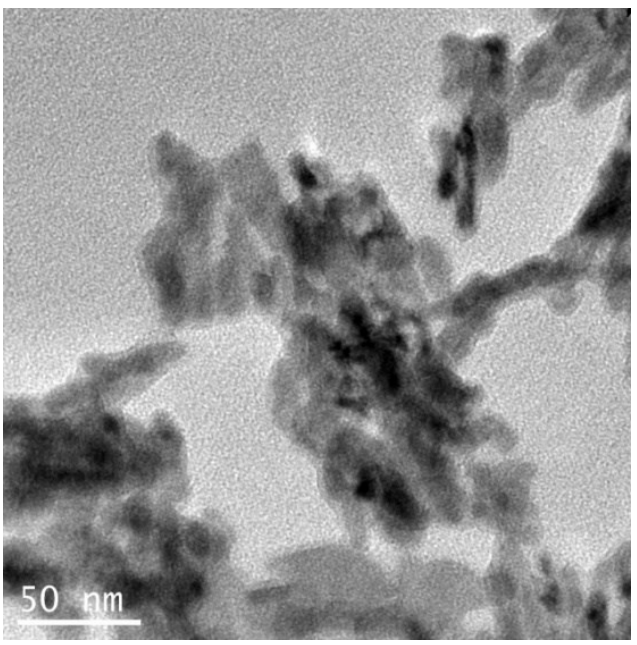

(b)

Figure 5.3.TEM images of (a) CuO-50 nano-rods and (b) HTCC-CuO-50 nano-rods.

\subsubsection{Nanoparticle and ACQ Retention in Wood Blocks}

PVP-stabilized $\mathrm{CuO}$ nanoparticles, HTCC-stabilized $\mathrm{CuO}$ nanoparticles, and unstabilized $\mathrm{CuO}$ nanoparticle suspensions were prepared and used to pressure-treat southern pine sap wood blocks $(19 \mathrm{~mm} \times 19 \mathrm{~mm} \times 19 \mathrm{~mm})$ according to the standard method of wood pressure treatment (AWPA E11-97). Target retentions ranged from 2.0 to $4.0 \mathrm{~kg} \mathrm{Cu}^{2+} / \mathrm{m}^{3}$ wood. Similarly, an ACQ solution control $\left(15 \mathrm{mg} \mathrm{Cu}^{2+} / \mathrm{mL}\right)$ was used to give the same target retention in southern pine sapwood blocks. The various nanoparticle suspensions appeared stable the actual delivery efficiency in the wood was 
usually $\sim 30-45 \%$ and only $48.1 \%$ for the ACQ control. The data do not suggest a significant impact of the polymer on the retention of the treated wood. The low retention is attributed to the high dilution of the suspensions needed to cover the wood blocks with fluid during the pressure-treatment process. The results are summarized in Table 5.1, showing retentions as both $\mathrm{kg}$ of $\mathrm{Cu}^{2+}$ per $\mathrm{m}^{3}$ wood and $\mathrm{CuO}$ per $\mathrm{m}^{3}$ wood. It should be noted that as a "legacy" of traditional wood treatment formulations in some circles copper content is often reported as if it exists as $\mathrm{CuO}$ even if it is added as $\mathrm{CuCO}_{3}$ or as a soluble copper salt. That is why in Table 5.1 we show ACQ as $\mathrm{Cu}^{2+}$ and as if it were $\mathrm{CuO}$ even though it is a soluble salt, and for comparison with our actual $\mathrm{CuO}$ nanoparticle retentions.

Table 5.1. Delivery efficiency and $\mathrm{Cu}^{2+} / \mathrm{CuO}$ retention in wood blocks.

\begin{tabular}{|c|c|c|c|}
\hline Sample & $\begin{array}{c}\text { Target retention } \\
\mathrm{Kg} / \mathbf{m}^{3} \\
\mathrm{Cu}^{2+} / \mathrm{CuO}\end{array}$ & $\begin{array}{c}\text { Delivery } \\
\text { Efficiency } \\
\%\end{array}$ & $\begin{array}{c}\text { Actual retention } \\
\mathrm{Kg} / \mathbf{m}^{3} \\
\mathrm{Cu}^{2+} / \mathrm{CuO}\end{array}$ \\
\hline $\begin{array}{l}\text { PVP-CuO-10 } \\
10 \mathrm{~nm} \text { Nanoparticles }\end{array}$ & $2.0 / 2.5$ & 44.8 & $0.896 / 1.12$ \\
\hline $\begin{array}{l}\text { HTCC-CuO-10 } \\
10 \mathrm{~nm} \text { Nanoparticles }\end{array}$ & $2.0 / 2.5$ & 44.0 & $0.880 / 1.10$ \\
\hline $\begin{array}{l}\text { CuO-10 } \\
10 \mathrm{~nm} \text { Nanoparticles }\end{array}$ & $2.0 / 2.5$ & 40.1 & $0.802 / 1.00$ \\
\hline CuO-50 & $2.0 / 2.5$ & 33.2 & $0.660 / 0.825$ \\
\hline $50 \mathrm{~nm}$ Nanoparticles & $4.0 / 5.0$ & 30.3 & $1.21 / 1.513$ \\
\hline $\begin{array}{l}\text { HTCC-CuO-50 } \\
50 \mathrm{~nm} \text { Nanoparticles }\end{array}$ & $2.0 / 2.5$ & 38.1 & $0.760 / 0.950$ \\
\hline ACQ Control & $2.0 / 2.5$ & 48.1 & $0.962 / 1.203$ \\
\hline
\end{tabular}

Despite the lower than expected delivery efficiency, nanoparticle penetration throughout the wood was verified by FESEM. PVP-CuO-10 nanoparticles were used to treat southern pine field stakes $(19 \mathrm{~mm} \times 19 \mathrm{~mm} \times 455 \mathrm{~mm})$ and a central section of the stake was sectioned out and visualized by FESEM to confirm effective penetration 
throughout the wood. Figure 5.4a shows PVP-CuO-10 nanoparticles absorbed onto the pit pairs from a region nearer the wood surface. Figure 5.4b is an image from deep in the interior of the wooden stake.

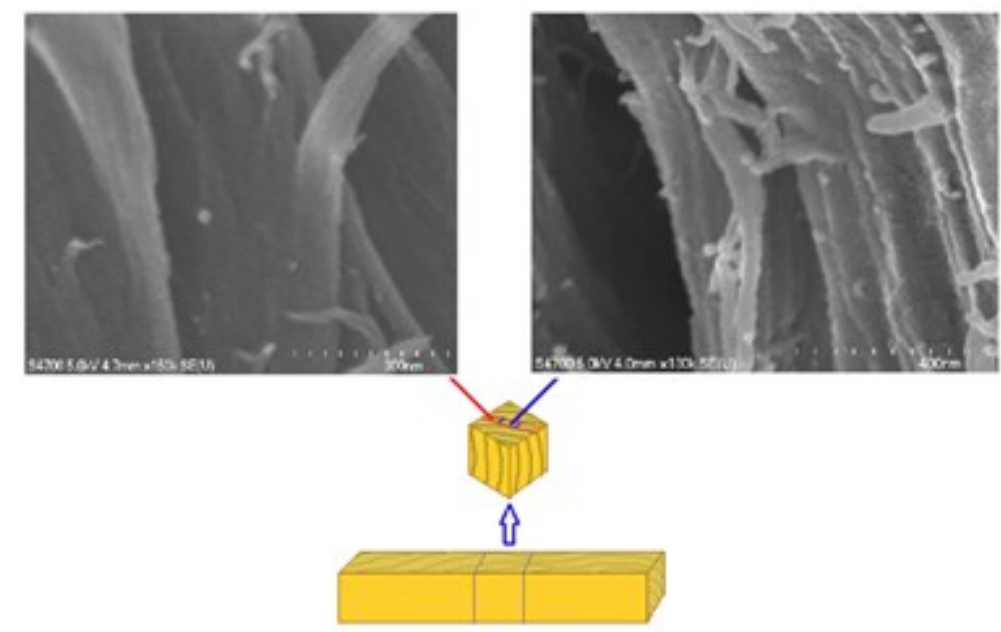

Figure 5.4. FESEM micrographs of wood sectioned from the center portion of a $19 \mathrm{~mm} \mathrm{x}$ $19 \mathrm{~mm}$ by $455 \mathrm{~mm}$ southern pine field stake showing PVP-CuO-10 nanoparticles (a) near the surface (scale bar $300 \mathrm{~nm}$ ) and (b) deep in the interior (scale bar $400 \mathrm{~nm}$ ).

\subsubsection{Leaching Tests}

The results of the AWPA standard leaching tests are shown in Figure $\mathbf{5 . 5}$ and reported as a percentage of $\mathrm{Cu}^{2+}$ based onthe original retention, regardless of if the copper was introduced as $\mathrm{CuO}$ or as ACQ. Several significant findings can be seen from these data.

First, based on our results, the size of the $\mathrm{CuO}$ nanoparticles is the most significant factor affecting leaching. Wood specimens treated with $10 \mathrm{~nm}$ nanoparticles (solid symbols in Figure 5.5), all leached significantly more copper than was released from the wood treated with $50 \mathrm{~nm} \mathrm{CuO}$ nanoparticles. Second, the polymer stabilizers used here (the weakly acidic HTCC and the weakly basic PVP) enhanced leaching compared to the unstabilized $\mathrm{CuO}$ nanoparticles, with unstabilized $\mathrm{CuO}-10$ nanoparticles leaching 12.3\% of its original retention, compared $15.6 \%$ of the original retention of the weakly acidic 
HTCC-CuO-10 nanoparticles and $14.3 \%$ of the weakly basic PVP-stabilized CuO-10 nanoparticles. The HTCC-capping also promoted leaching in the CuO-50 nanoparticles (hollow triangle symbols in Figure 5.5) when compared to the ACQ salt (hollow black diamonds) The unstabilized $\mathrm{CuO}-50 \mathrm{~nm}$ nanoparticles leached only approximately $5 \%$ of the copper compared to $6.7 \%$ for the ACQ control and approximately $7 \%$ for the HTCC-stabilized CuO-50 nanoparticles.

We are aware of only one other publication, by Kartal and co-researchers, that addresses leaching of copper oxide nanoparticles (6) and those authors found favorable leach resistance of copper and zinc oxide nanoparticles compared to their more soluble equivalents. They used commercial nanoparticle formulations so the exact composition, nanoparticle size, and uniformity of size were not provided. However, given that commercial formulations all appear to use nanoparticles that are larger than $\mathrm{CuO}-10$, our results are consistent with those reported findings, which are that nanoparticles leach slightly less than soluble salts, and polymer stabilizers (here HTCC and PVP) facilitate leaching. However, the results with $\mathrm{CuO}-10$ nanoparticles indicate that nanoparticle size is a factor to be considered in leaching. We can only speculate that the reason the $\mathrm{CuO}-10$ nanoparticles leach so much more than the CuO-50 nanoparticles is simply that their small size makes them more mobile than $\mathrm{CuO}-50$ and so the solid phase nanoparticles leach out of the wood more readily than solubilized $\mathrm{Cu}^{2+}$ ions or $50 \mathrm{~nm}$ nanoparticles. It is also likely that $\mathrm{CuO}-10$ and $\mathrm{CuO}-50$ nanoparticles were retained on the surface and these are contributors to leachate residues. Whatever the reason may be, the final result from this small study remains the same: $\mathrm{CuO}-10 \mathrm{~nm}$ nanoparticles leach significantly more than $\mathrm{CuO}-50 \mathrm{~nm}$ nanoparticles. 


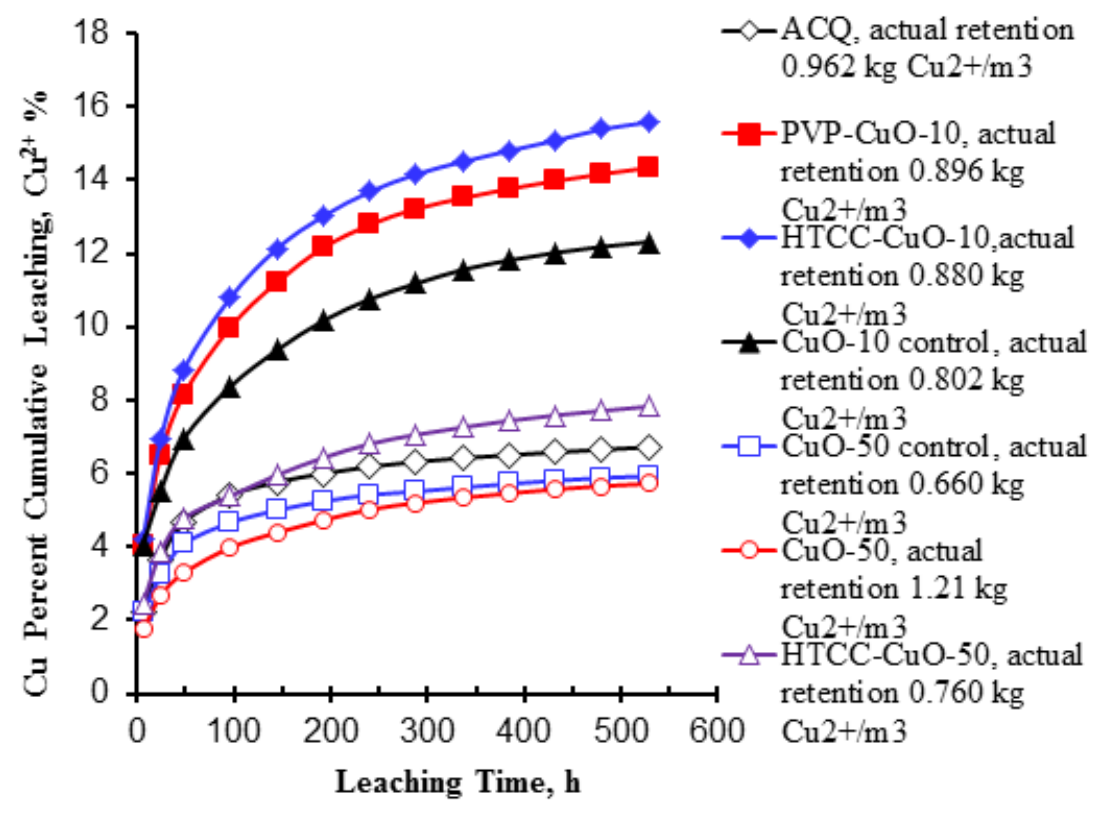

Figure 5.5. Cumulative leaching of $\mathrm{Cu}^{2+}$ from wood blocks (plotted as a percent of original retention).

We also wanted to know if the nanoparticles themselves were leaching, TEM micrographs (Fig.5.6a) the presence of significant numbers of nanoparticles in the leachate and EDS analysis (Fig.5.6b) confirmed that these were copper. We suspect that the polymer stabilizers increased the leaching of nanoparticles, perhaps simply by preventing aggregation of nanoparticles within the wood, making them more mobile, but we were not able to prove this. A similar trend was observed for HTCC-CuO-10 nanoparticles with the confirming TEM and EDS given in Figure 5.6c and 5.6d, respectively. 


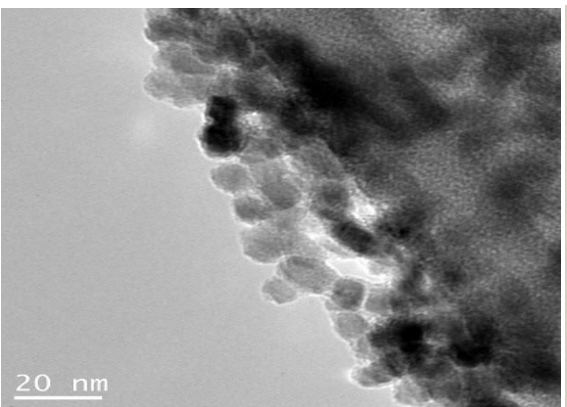

(a)

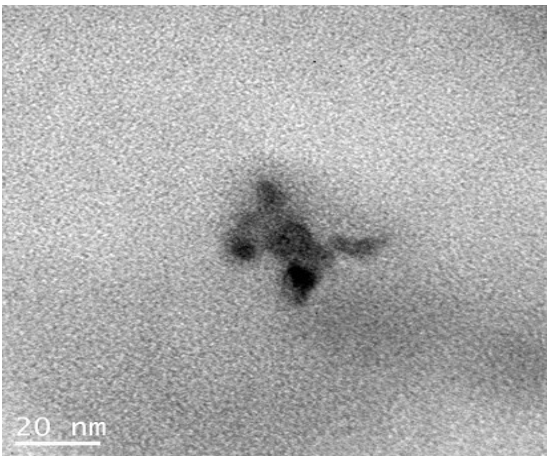

(c)

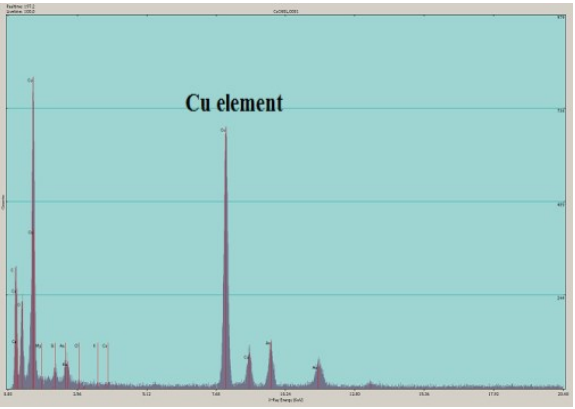

(b)

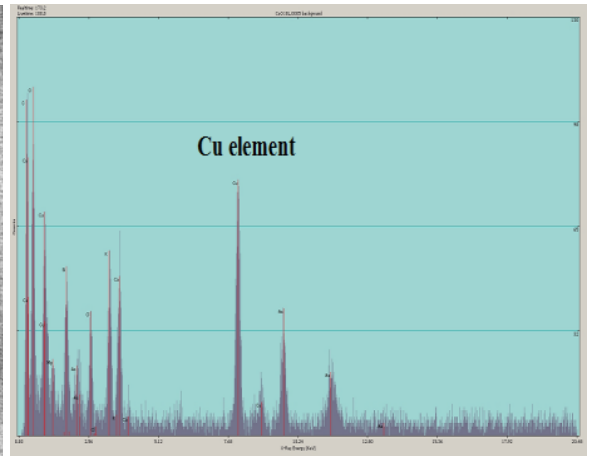

(d)

Figure 5.6. TEM images of $\mathrm{CuO}$ nanoparticles found in leachate from polymer-stabilized CuO-10 nanoparticle and unstabilized CuO-10 nanoparticle treated wood blocks and verified by EDS to be $\mathrm{CuO}$.(a) $\mathrm{CuO}-10$ nanoparticles in leachate burst released after the first 24 h; (b) its EDS spectrum; (c) HTCC-CuO-10 nanoparticles in leachate collected between $24 \mathrm{~h}$ and $48 \mathrm{~h}$; (d) its EDS spectrum.

The significance of these results lies in the perception that smaller nanoparticles are desirable as a means to enhance biological effect, and that less soluble forms of wood preservatives are thought of as a means to reduce biocide leaching. However, this study shows that smaller $(10 \mathrm{~nm})$ nanoparticles are more prone to leaching than a soluble copper salt. Besides the still unresolved concerns of nanoparticles released into the environment, increased leaching is also undesirable because loss of the preservative reduces the longevity of the preserved wood. Another significant result was that $50 \mathrm{~nm}$ 
nanoparticles were less susceptible to leaching than the $10 \mathrm{~nm}$ nanoparticles, indicating that if enhanced efficacy is achieved with smaller nanoparticle size then there will be an optimal size that achieves the best efficacy without increased susceptibility to leaching. Finally, this work showed that both a weakly acidic (HTCC) and a weakly basic (PVP) polymer stabilizer enhanced the leaching of the $10 \mathrm{~nm}$ nanoparticles, and HTCC also slightly enhanced the leaching of the $50 \mathrm{~nm}$ nanoparticles. PVP was not evaluated with the larger nanoparticles, though the fact that wood is slightly acidic suggests PVP, or an even more basic stabilizer, might give better results than we observed. Therefore, nanoparticle stabilizers and/or post-conditioning protocols should be tested with different sizes of nanoparticles, to determine if they are as effective with smaller nanoparticles as they are with larger ones, to determine if they reduce leaching or perhaps even promote leaching of the nanoparticles.

\subsubsection{Biological Efficacy against G. trabeum in Soil Jar Decay Tests}

The final objective of this work was to determine if $10 \mathrm{~nm}$ nanoparticles, due to their very high surface area, might afford protection against fungal attack at very low treating levels. Wood specimens were treated with PVP-CuO-10 and ACQ as a control. The retention efficiency was lower than expected so the actual biocide content was lower than intended, at $0.127 \mathrm{~kg} \mathrm{Cu}^{2+} / \mathrm{m}^{3}$ wood for the PVP-CuO-10 and $0.188 \mathrm{~kg} \mathrm{Cu}^{2+} / \mathrm{m}^{3}$ wood for the ACQ when the target retention was $0.32 \mathrm{~kg} / \mathrm{m}^{3}$. No significant biological effect was evident at this level, nor was any significant difference detected on comparing the ACQ and PVP-CuO-10 nanoparticles (Fig. 5.7). Higher retentions were not attempted because of the significant leaching of the polymer-stabilized and unstabilized CuO-10 nanoparticles. 


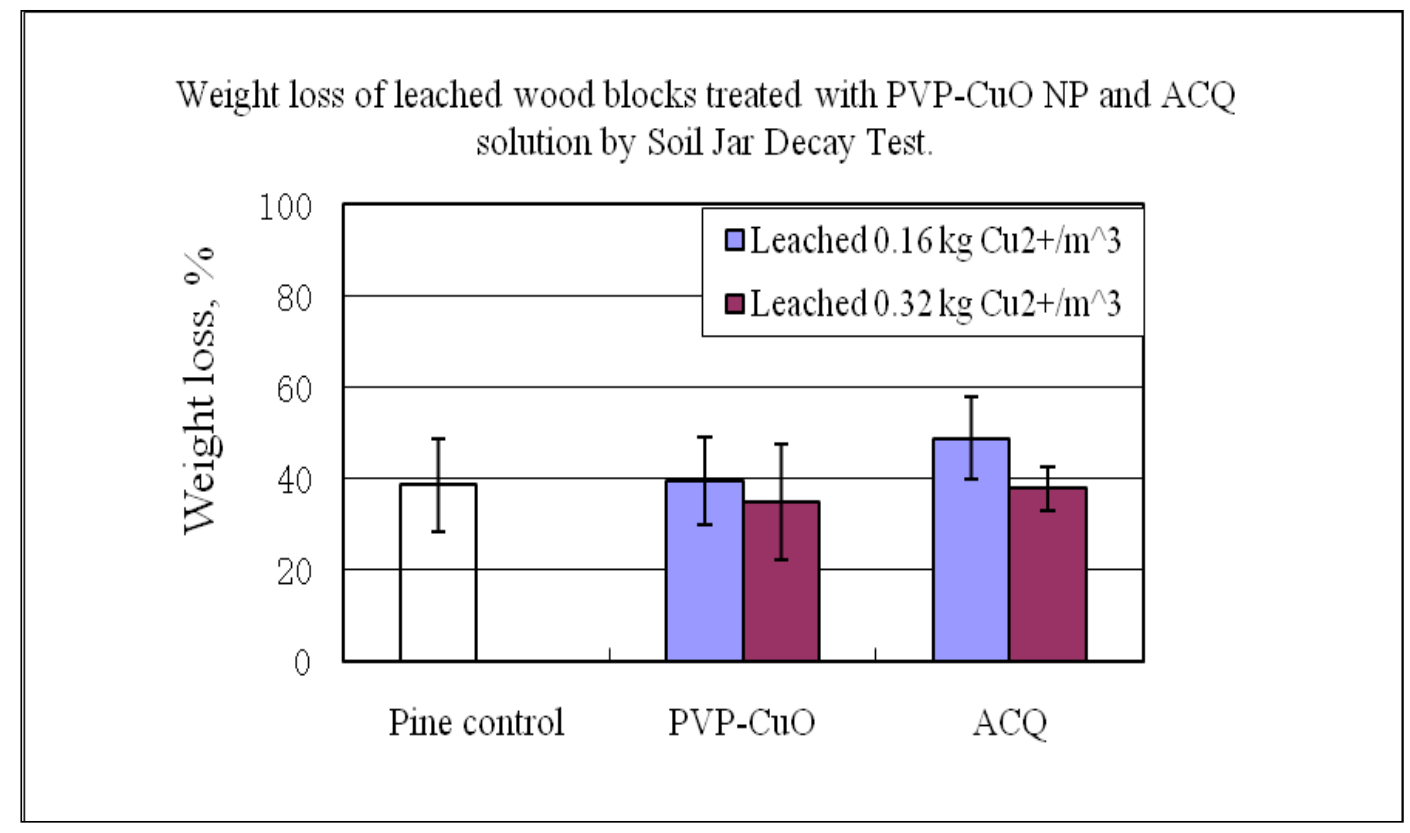

(a)

Weight loss of unleached wood blocks treated with PVP-CuO NP and ACQ solution by Soil Jar Decay Test.

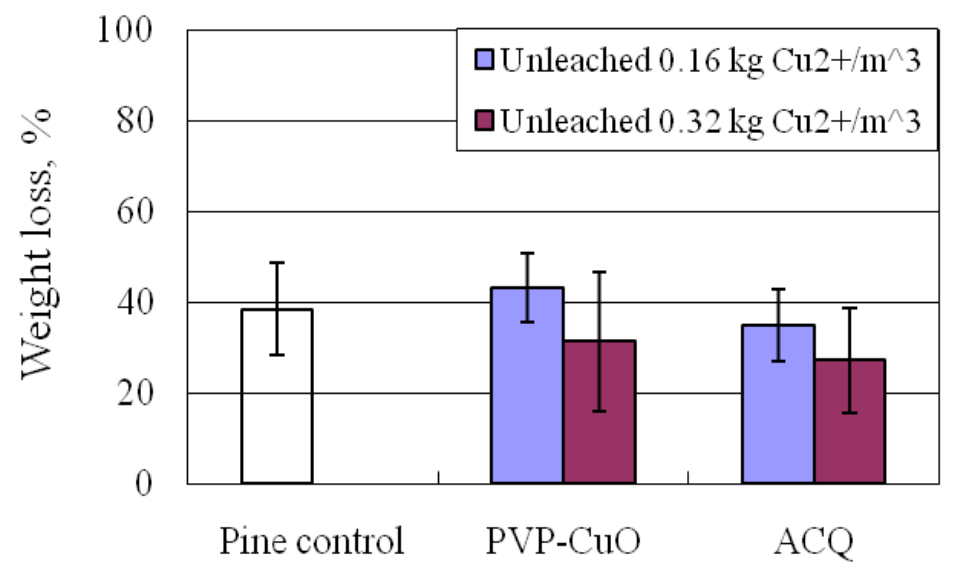

(b)

Figure 5.7. Weight loss of southern pine sapwood treated with PVP-CuO-10 nanoparticles and ACQ measured using soil jar decay tests of (a) leached wood blocks, and (b) unleached wood blocks. The target retentions shown are based on $\mathrm{Cu}^{2+}$. 


\subsection{Conclusion}

This research produced three major findings concerning leaching of copper from nanoparticle-treated wood, though it must be noted that we used small sample sizes. These are: nanoparticles are leached in significant numbers from pine sapwood treated with $10 \mathrm{~nm}$ or $50 \mathrm{~nm}$ copper oxide nanoparticles, that nanoparticle size is a significant factor in the relative abundance of leaching, and that polymer stabilizers enhance nanoparticle leaching. Due to the high potential error inherent in trying to separate nanoparticles of this size, we did not determine if all the released copper was in the form of nanoparticles, but both the total leaching and the abundance of nanoparticle leaching was significantly higher for $\mathrm{CuO}-10$ nanoparticles than for $\mathrm{CuO}-50$ nanoparticles. We found that both weakly acidic (HTCC) and weakly basic (PVP) polymer stabilizers increased nanoparticle leaching compared to the un-stabilized nanoparticles of the same size. An attempt was made to determine if the $\mathrm{CuO}-10$ nanoparticles had greater biological effect than the larger $\mathrm{CuO}-50$ nanoparticles. However, our nanoparticle retention levels were below the minimum required for any significant effect to be determined, so we did not answer that question. Although neither the environmental fate nor negative environmental impact of different "nano copper" based wood preservatives are yet known, the major implications from this work are that larger "nano copper" nanoparticles would be more advantageous than otherwise similar treatments using smaller "nano copper" because the greater leaching will likely leave the wood less well-protected. 


\section{Main Findings in This Chapter:}

Copper oxide nanoparticles with uniform size distribution of $\sim 10 \mathrm{~nm}$ and $\sim 50 \mathrm{~nm}$ were prepared by thermal hydrolysis with or without presence of polymer stabilizers. Leaching test of copper oxide nanoparticle-treated wood showed that copper leached more quantity from $\sim 10 \mathrm{~nm}$ treated wood than soluble copper salt (ACQ) control, but $\sim 50$ $\mathrm{nm}$ nanoparticle treated one leached slightly less copper than ACQ control. The higher quantity of copper leaching from nanopartcile-treated wood was confirmed by TEM and EDS analysis that the nanoparticles themselves were leached from treated wood. It was found that both weakly acidic (HTCC) and weakly basic (PVP) polymer stabilizers increased nanoparticle leaching compared to the un-stabilized nanoparticles of the same size. Biological activity of $\sim 10 \mathrm{~nm}$ copper oxide nanoparticle was compared with ACQ-solution treated wood, but nanoparticle retention levels were below the minimum required for any significant effect to be determined. The major implications from this work are that reasonably larger copper oxide nanoparticles would be more advantageous than otherwise similar treatments using smaller nanoparticles because the greater leaching from smaller nanoparticle treatment would sacrifice wood protection period. Our research shows the trends that nanoparticle size is main factor to affect copper leaching. 


\section{Acknowledgements:}

The author thanks the publisher (John Wiley and Sons) for offering permission without charge to reuse this published article in my Dissertation. Permission License Number: 3152331180412.

This work was funded by the United States Environmental Protection Agency Science to Achieve Results (STAR) Program, Grant Number GR832371 and by The Department of Chemistry at Michigan Technological University. The authors thank Dr. Peter Laks, Mr. Glen Larkin, and Mr. Erik Keranen, at MTU School of Forest Resources and Environmental Science, for preparing and pressure treating the wood blocks and stakes used in this work, and Mr. Owen Mills, Department of Materials Science and Engineering at MTU for the TEM images. The authors also thank Dr. Ning Chen for the FESEM images. 


\section{References}

[1] A.Q. Nyrud, A. Roos, M. Rodbotten, Can J. For. Res. 2008, 38, 1385-1396.

[2] M. H. Freeman, C.R. McIntyre, Forest Products Journal2008, 58, 6-27.

[3] P. Evans, H. Matsunaga, M. Kiguchi, Nature Nanotech2008, 3, 577.

[4] J. Hansen, G. A. Kimbrell, Letter to Stehen A. Owens, Office of Chemical Safety and Pollution. International Center for Technology Assessment2010.

[5] P. A. Heiden, Personal Communication, Osmose Technical Representative2010.

[6] S.N. Kartal, F. Green III, C. A. Clausen, International Biodeterioration and Biodegradation 2009, 63, 490.

[7] K. Sissel, National Science Foundation funds research into environmental fate of nanoparticles. Chemical Week 2004, 66(28), 7.

[8] A. B. Boxall, K. Tiede, Q. Chaudhry, Nanomedicin2007, 2(6), 919.

[9] I. K. Koponen, K. A. Jensen, T. Schneider, Journal of Exposure Science and Environmental Epidemiology2011, 21(4), 408.

[10] H. L. Karlsson, P. Cronholm, J. Gustafsson, L. Moller, Chemical Research In Toxicology2008, 21(9), 1726.

[11] H. W. Richardson, R. L. Hodge, Particulate wood preservative and method for producing same, EP1651401B1, European patent 2009.

[12] S. W. Jeong, S. D. Kim, J. Environ. Monit.2009, 11( 9), 1595.

[13] T. Ben-Moshe, I. Dror, B. Berkowitz, Chemosphere2010, 81, 387.

[14] A. R. Petosa, D. P. aisi, I. R. Quevedo, M. Elimelech, N. Tufenkji, Environ. Sci. Technol. 2010, 44, 6532.

[15] T. M. Benn, P. Westerhoff, Environ. Sci. Technol. 2008, 42(11), 4133.

[16] P. Gajjar, B. Pettee, D.W. Britt, W. Huang, W.P. Johnson, A. J. Anderson, J. Biological Engineering2009,3(9),1.

[17] N. Jones, B. Ray, K.T. Ranjit, A.C. Manna, Microbial lett.2008, 279(1), 71. 
[18] J. Zhang, D. Kamden, Holzforschung2000, 54, 119.

[19] J. Zhu, D. Li, H. Chen, X. Yang, L. Lu, X. Wang, Materials Letters 2004, 58, 3324.

[20] S. H. Lim, S. M. Hudson, Carbohydrate2004, 339, 313.

[21] T. Kida, T. Oka, M. Nagano, Y. Ishiwata, X. Zheng, Journal of the American Ceramic Society2007, 90(1), 107.

[22] H. Zhang, U. Siegert, R. Liu, W. Cai, Nanoscale Res Lett.2009, 4, 705.

[23] American Wood-Preservers Association Book of Standards 1998, Standard Method of Testing Wood Preservatives by Laboratory Soil-Block Culture E10-91. Branbury Texas, E10-91, 375. 


\section{Chapter 6}

\section{Future Work}

In chapter 2 and 3, the self-assembly study of peptide-functionalized amphiphilic copolymer confirmed the hypothesis that ionic complementary peptides could direct peptide-functionalized polymeric nanoparticles to self-assemble into nanoparticle fibers and 3D scaffolds by ionic complementary self-assembly between two oppositely charged $\beta$-sheet peptides. The 1D nanoparticle fiber has a length range from tens to hundreds micrometer. Smaller nanoparticle self-assemble into more compact and stable 3D scaffolds than bigger nanoparticles, but with less porosity.

The self-assembly of the designed peptides (P1: H2N-TTTT-AEAEAEAE-amide and P2: H2N-TTTT-AKAKAKAK-amide) in aqueous solution (PBS and neutral deionized water) were also studied. Results showed that reasonable salt $(\mathrm{NaCl})$ concentration facilitated the peptide self-assembly to form a stable membrane with a distinct morphology, but no nanofiber structure was observed. And if the self-assembled peptide membrane was washed with deionized water to remove salt, the morphology was altered and gavean amorphous aggregate, which is attributed to high water solubility of the designed peptides (P1 and P2).

Based on the analysis of the data from these studies, in future work, several aspects can be further conducted to improve the self-assembly system for practical application.

1. Reduce the spacer parts of both $\mathrm{P} 1$ and $\mathrm{P} 2$ from four units to two units, and increase self-assembly units from 8 to 12 , so the peptide structure for P1 and P2 are shown as: $\quad \mathrm{P} 1 \quad\left(\mathrm{H}_{2} \mathrm{~N}-\mathrm{TT}-\mathrm{AEAEAEAEAEAE-amide}\right)$ and $\mathrm{P} 2$ $\left(\mathrm{H}_{2} \mathrm{~N}-\mathrm{TT}\right.$-AKAKAKAKAKAK-amide). Reduce threonine $(\mathrm{T})$ and increase self-assembly units, alanine (A), glutamic acid (E) and lysine (K) can increase the stability of the self-assembled $\beta$-sheets structure, and also can perform self-assembly in a lower peptide concentration. Or we can substitute valine (V) or leucine (L) for alanine (A) to increase the 
hydrophobicity of P1 and P2, but still keep same self-assembly unit numbers to form P1: H2N-TTTT-VEVEVEVE-amide and P2: H2N-TTTT-VKVKVKVK-amide. So self-assembly of P1 and P2 with new sequence can also form more stable $\beta$-sheets structure at lower peptide concentration.

2. Synthesize $\mathrm{P} 2$ using another type of resin to keep the amine protection group after P2 is cleaved from resin, so that when P2 is used to couple with copolymer, only the peptide amine terminal is reacted with the carboxylic acid group of copolymer terminal. Because lysine also has an amine group on the side chain, without a protection group, some coupling will occur at thelysine amine to form a branched structure (Figure 6.1). Such a branch structure detrimental to the subsequent assembly between P1 and P2.

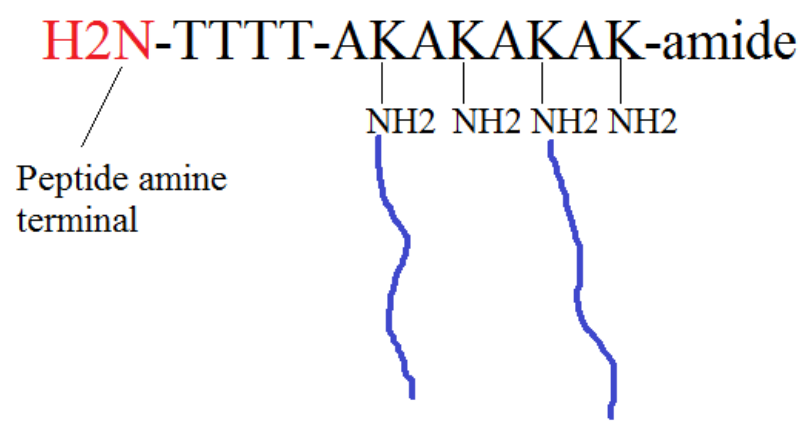

Figure 6.1. Branch structure if copolymer is coupled with lysine amine groups but not the peptide amine terminal.

3. Cytotoxicity tests showed that NIH3T3 cells did not effectively adhere to the self-assembled 2D nanoparticle membrane surface. In order to increase cell adhesion to scaffolds, both 2D and 3D, the nanoparticle surface probably needs modification with some types of cell adhesion peptides or other type of receptor to increase cell adhesion. The surface modification can be done by functionalizing cell receptors (i.e. cell adhesion peptides) with vinyl groups and then copolymerizing them with hydrophilic monomer to build up a hydrophilic block,so the cell receptors are formed on the hydrophilic shell of the 
nanoparticle surface. The nanoparticle surface functionalization with receptors is shown in Figure 6.2.
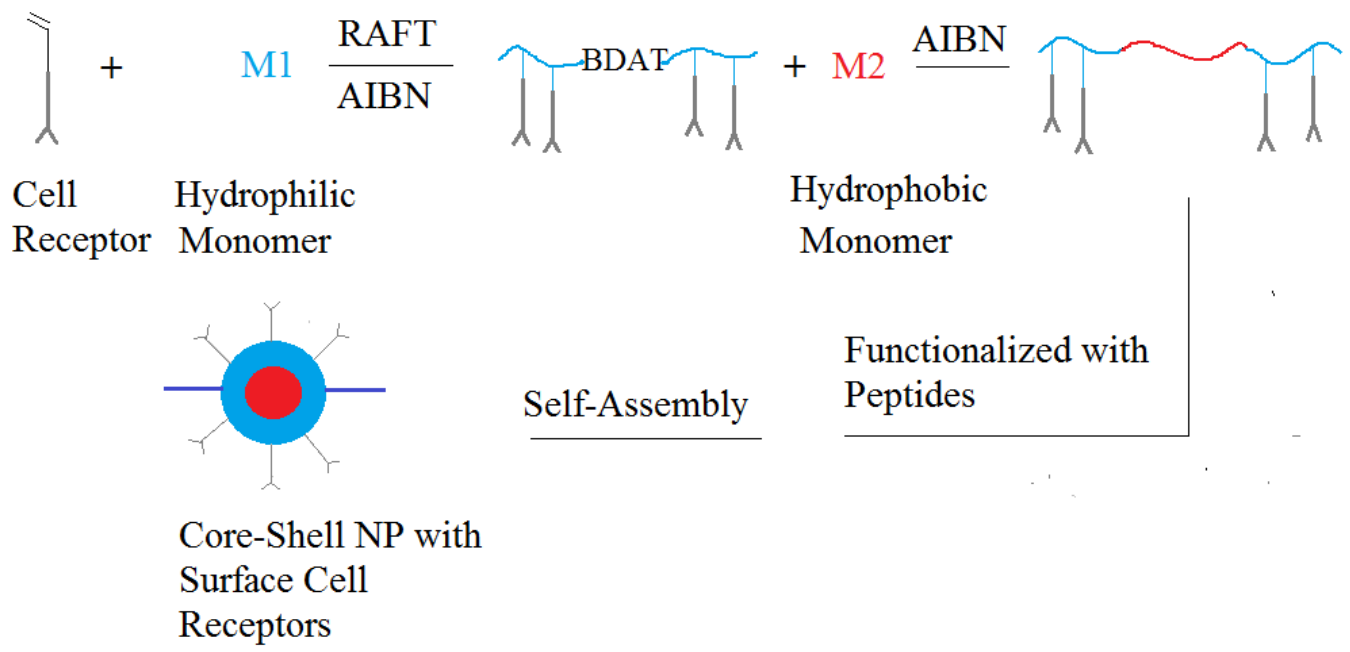

Figure 6.2. Cartoon shows covalently incorporate cell receptors in hydrophilic block to self-assemble into core-shell nanoparticle with cell receptors on nanoparticle surface.

Such surface functionalized nanoparticles can interact with cell surfaces to increase cell adhesion. If the self-assembled nanoparticle scaffold is mixed with a specific cell type, it is possible that the cells themselves will also interact with nanoparticles to form in-situ self-assembled 3D scaffolds. Then the incorporated drugs released from the nanoparticles can stimulate cell proliferation and migration to guide cells to develop into the desired mature cell type for specific tissue regeneration applications.

4. Techniques for determining bioactivity are needed if bioactive drugs are incorporated and released from the designed solid nanoparticles. Also, specific quantitative analysis techniquesare needed to determine the release rate of bioactive drugs from nanoparticle. These two steps are important if this self-assembled nanoparticle scaffold is used to load growth factors for tissue regeneration because most growth factors 
are required to remain bioactive so its functionality will stimulate cell activities to work normally. If multiple growth factors are simultaneously loaded, maybe a more advanced technique is required to determine release rate and bioactivities of each growth factor.

5. In vitro cell tests with self-assembled scaffolds with or without loading of drugs also must be done. This is important to know the cell growth behavior inside the scaffolds, and also monitor the stability of the scaffolds when culturing in cell media.

6. Possible toxicity of decomposed components from scaffolds must be tested before the scaffolds are tested in vivo.

The above work was not done by the author because offinancial and time limitations, but the work described above is expected to improve the design and efficacy of the scaffolds and achieve better control of the self-assembled structure. 


\section{Appendix A}

\section{Supplementary Data for Chapter 2}

Studies of Novel Self-Assembly Structures from Peptide-Functionalized

Polymeric Micro- and Nanoparticles for Tissue Engineering

Xiaochu Ding, Jagadeesh Janjanam, Ashutosh Tiwari, Marty Thompson, Patricia A. Heiden*,

Department of Chemistry, Michigan Technological University, Houghton, MI 49931, USA

*Corresponding author: +1 906487 3452; Fax: +1 9064872061.

Email address: paheiden@mtu.edu (P.A. Heiden).

1. MALDI-DOF MS analysis of the designed peptides (a) P1 and (b) P2.

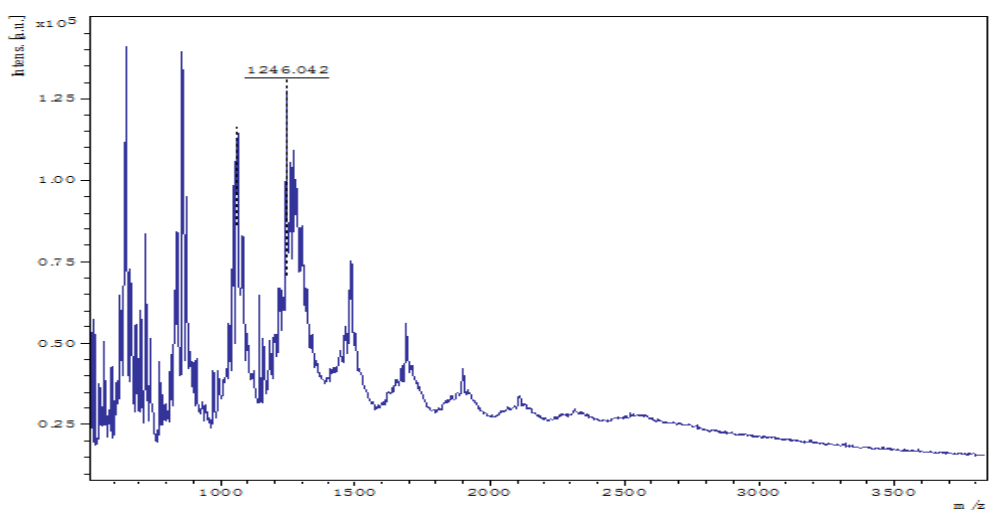

(a) 


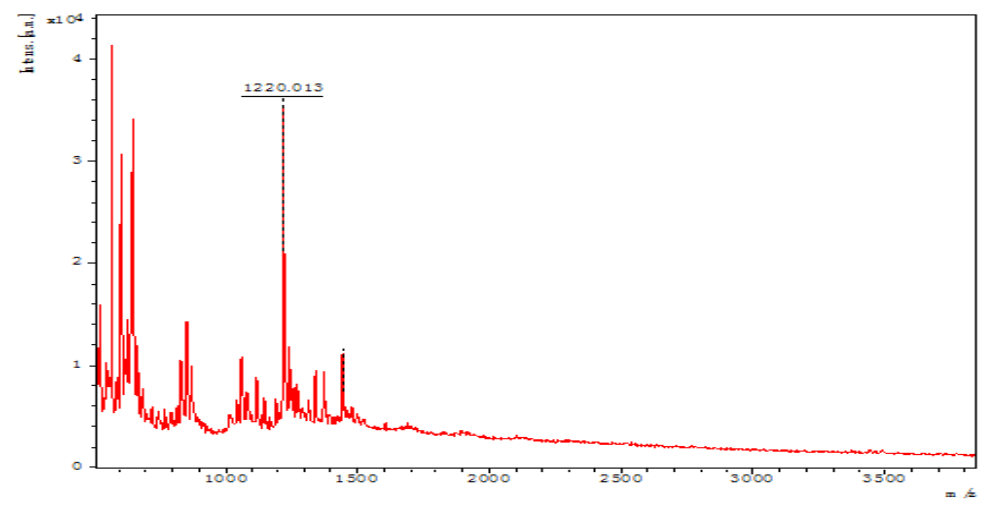

(b)

Figure 2.1S. MALDI-TOF MS analysis of the designed peptides.(a) P1 $\left(\mathrm{H}_{2} \mathrm{~N}\right.$-TTTT-AEAEAEAE-amide) and (b) P2 ( $\mathrm{H}_{2} \mathrm{~N}$-TTTT-AKAKAKAK-amide).P1 and P2 have theoretical molecular weight of 1223.25 and 1219.45 Da. MALDI-TOF MS analysis shows strong MS peak at $1246.42\left[\mathrm{M}(\mathrm{P} 1)+\mathrm{Na}^{+}\right]$and $1220.01\left[\mathrm{M}(\mathrm{P} 2)+\mathrm{H}^{+}\right] \mathrm{Da}$.

The Figure 2.1S shows the spectra of molecular weight of the designer peptides, P1 (H2N-TTTT-AEAEAEAE-amide) and P2 (H2N-TTTT-AKAKAKAK-amide), by MALDI-TOF MS analysis.

\section{Synthesis of $\mathbf{S}, \mathbf{S}^{\prime}$-bis $\left(\alpha, \alpha^{\prime}\right.$-dimethylacetic acid) trithiocarbonate (BDAT)}

The RAFT chain transfer agent, BDAT, was synthesized by adding carbon disulfide (2.74 g, $0.036 \mathrm{~mol})$, chloroform $(10.75 \mathrm{~g}, 0.09 \mathrm{~mol})$, acetone $(5.23 \mathrm{~g}, 0.09 \mathrm{~mol})$ and tetrabutylammonium hydrogen sulfate $(0.241 \mathrm{~g}, 0.71 \mathrm{mmol})$ into room temperature ligroin $(12 \mathrm{~mL})$ in a $250 \mathrm{~mL}$ round bottom flask. The flask was equipped with an efficient condenser and a magnetic stir bar and maintained under a nitrogen atmosphere. Then $\mathrm{NaOH}$ (50 wt.\% in $\mathrm{H}_{2} \mathrm{O}$ ) (20.16 g, $0.252 \mathrm{~mol}$ ) was added drop-by-drop to the reaction mixture over $1.5 \mathrm{~h}$ at a rate sufficient to keep the reaction temperature below $25{ }^{\circ} \mathrm{C}$. After the addition was completed the reaction was continued overnight at $22-25{ }^{\circ} \mathrm{C}$. Then deionized $\mathrm{H}_{2} \mathrm{O}(90 \mathrm{~mL})$ was added to dissolve the yellow solids. This was followed by addition of $\mathrm{HCl}_{\text {conc. }}(12 \mathrm{~mL}$, via syringe) to acidify the aqueous solution and produce yellow crystalline solids. The crude product was collected by vacuum filtration and washed several times with deionized $\mathrm{H}_{2} \mathrm{O}$. After drying under reduced pressure at room temperature for $12 \mathrm{~h}, 3.26 \mathrm{~g}$ of crude BDAT product was obtained. The product was purified by recrystallization in toluene/acetone $(3: 1 \mathrm{v} / \mathrm{v})$ to afford a yellow crystalline product. 
3. ${ }^{1} \mathrm{H}-\mathrm{NMR}$ analysis of PHEMA-b-12PMMA-b-PHEMA,

PHEA-b-12PMMA-b-PHEA and their block ratio calculation equation

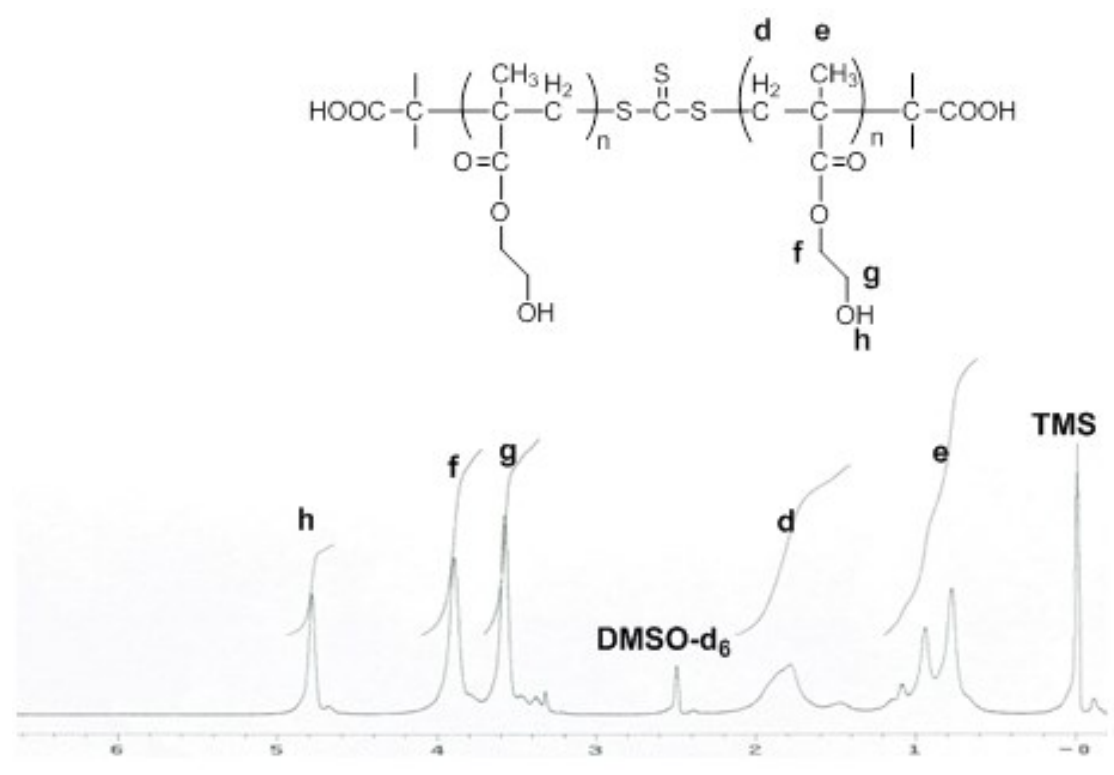

(a)

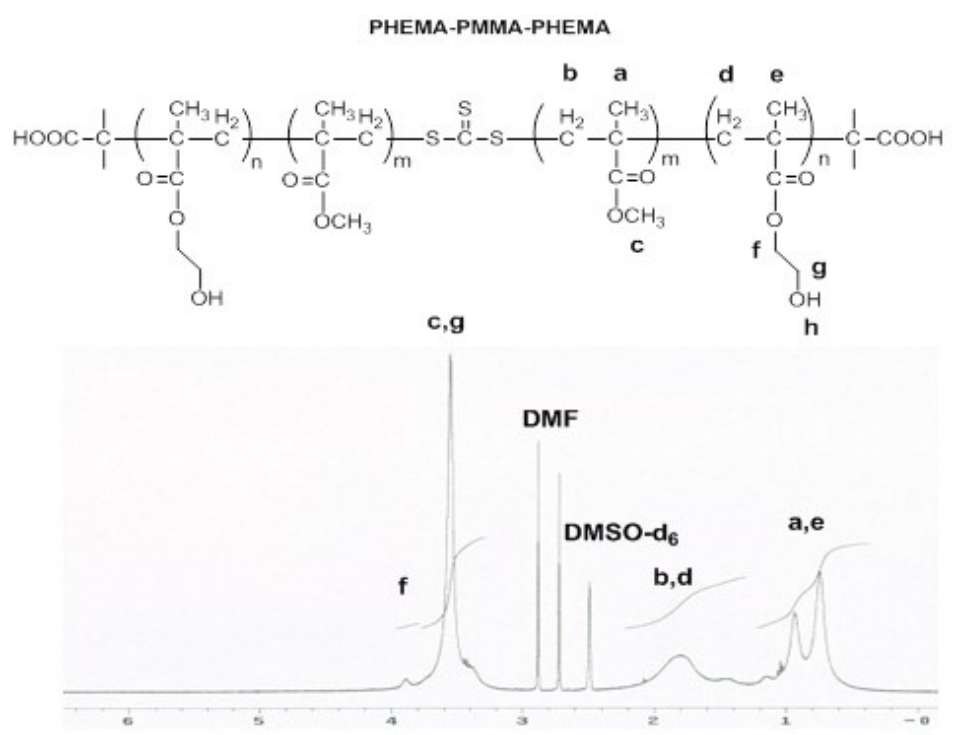

(b) 
Figure 2.2S. ${ }^{1} \mathrm{H}-\mathrm{NMR}$ spectra (in DMSO-d6) of (a) the synthesized hydrophilic polymer of PHEMA and (b) tri-block copolymer of PHEMA-b-12PMMA-b-PHEMA by RAFT polymerization.

Proton peak assignments of hydrophilic block (PHEMA) and amphiphilic block copolymer (PHEMA-b-12PMMA-b-PHEMA). PHEMA Block (in DMSO-d6, 8 ):

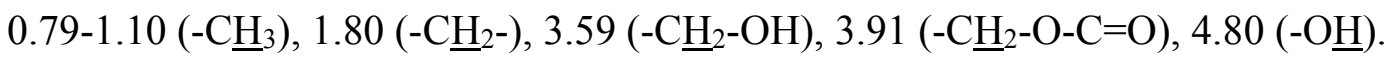

PHEMA-b-PMMA-b-PHEMA(in DMSO-d6, $\delta): 0.72-1.13 \quad\left(-\mathrm{CH}_{3}\right), \quad 1.42-1.77$ $\left(-\mathrm{C}_{2}-\right), 3.53\left(-\mathrm{OC}_{3} \underline{H}_{3}\right.$, from PMMA block, also contains $-\underline{\mathrm{CH}}_{2}-\mathrm{OH}$ from the PHEMA block), $3.87\left(-\underline{\mathrm{H}}_{2}-\mathrm{O}-\mathrm{C}=\mathrm{O}\right)$.

The integrated peak area of $\sigma: 3.91\left(-\mathrm{C}_{2}-\mathrm{O}-\mathrm{C}=\mathrm{O}\right.$ from PHEMA block) and $\sigma: 3.53$ (-OC $\underline{H}_{3}$, from PMMA block, also contains $-\underline{\mathrm{CH}}_{2}-\mathrm{OH}$ from the PHEMA block) are used to determine actual block ratio of PHEMA to PMMA $\left(\mathrm{X}_{\mathrm{N}}: \mathrm{X}_{\mathrm{M}}\right)$ to be 1:11 based on below equation.

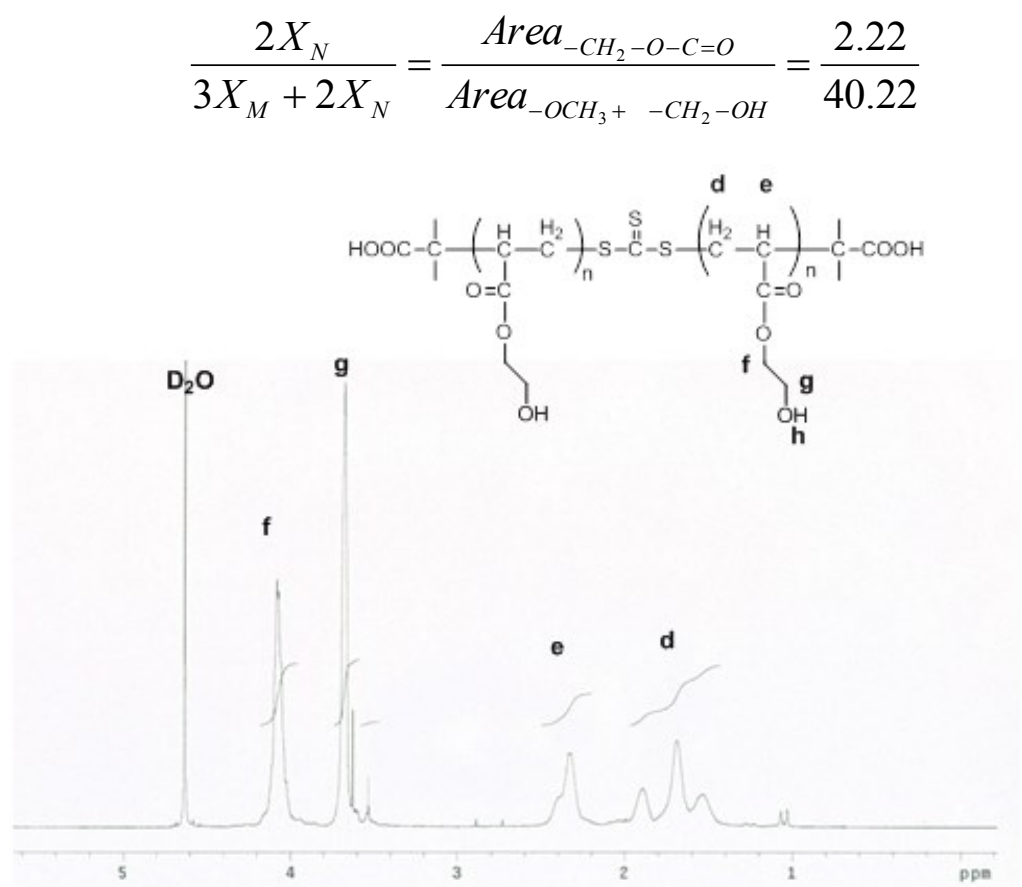

(a) 


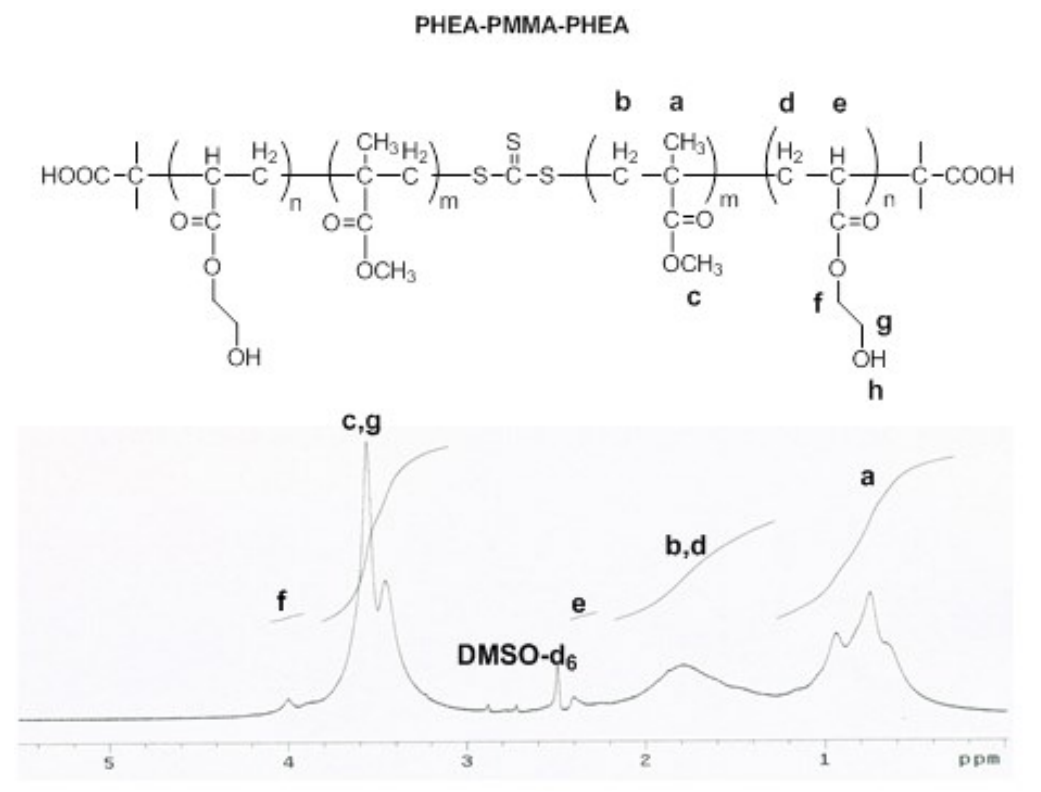

(b)

Figure 2.3S. ${ }^{1} \mathrm{H}-\mathrm{NMR}$ spectra (in DMSO-d6) of (a) the synthesized hydrophilic PHEA and (b) tri-block copolymer of PHEA-b-12PMMA-b-PHEA by RAFT polymerization.

Proton peak assignments of hydrophilic block (PHEA) and amphiphilic block copolymer (PHEA-b-12PMMA-b-PHEA).

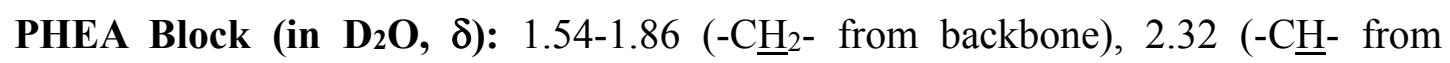
backbone), $3.53(-\mathrm{O} \underline{\mathrm{H}}), 3.68\left(-\mathrm{C}_{2}-\mathrm{OH}\right), 4.08\left(-\underline{\mathrm{C}}_{2}-\mathrm{O}-\mathrm{C}=\mathrm{O}\right)$.

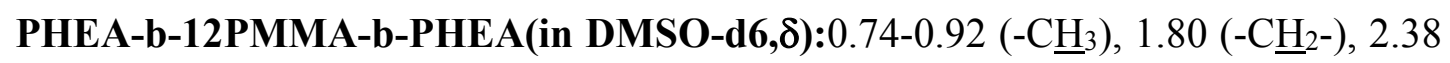

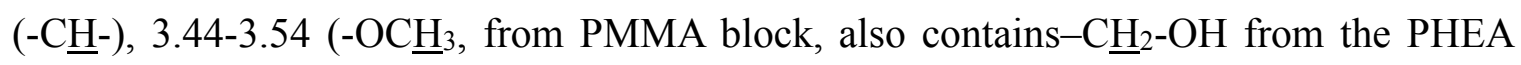
block), $3.98\left(-\underline{\mathrm{C}}_{2}-\mathrm{O}-\mathrm{C}=\mathrm{O}\right)$.

The integrated peak area of $\sigma: 3.98\left(-\underline{\mathrm{C}}_{2}-\mathrm{O}-\mathrm{C}=\mathrm{O}\right.$ from PHEA block $)$ and $\sigma$ : 3.44-3.54 (-OC $\underline{\mathrm{H}}_{3}$, from PMMA block, also contains $-\mathrm{C}_{2}-\mathrm{OH}$ from the PHEA block) are used to determine actual block ratio of PHEA to PMMA $\left(\mathrm{X}_{\mathrm{N}}: \mathrm{X}_{\mathrm{M}}\right)$ to be 1:8.7 based on below equation.

$$
\frac{2 X_{N}}{3 X_{M}+2 X_{N}}=\frac{\text { Area }_{-\mathrm{CH}_{2}-\mathrm{O}-\mathrm{C}=\mathrm{O}}}{\text { Area }_{-\mathrm{OCH}_{3}+}{ }_{-\mathrm{CH}_{2}-\mathrm{OH}}}=\frac{2.53}{35.62}
$$




\section{Coupling peptides to copolymers by FTIR analysis}

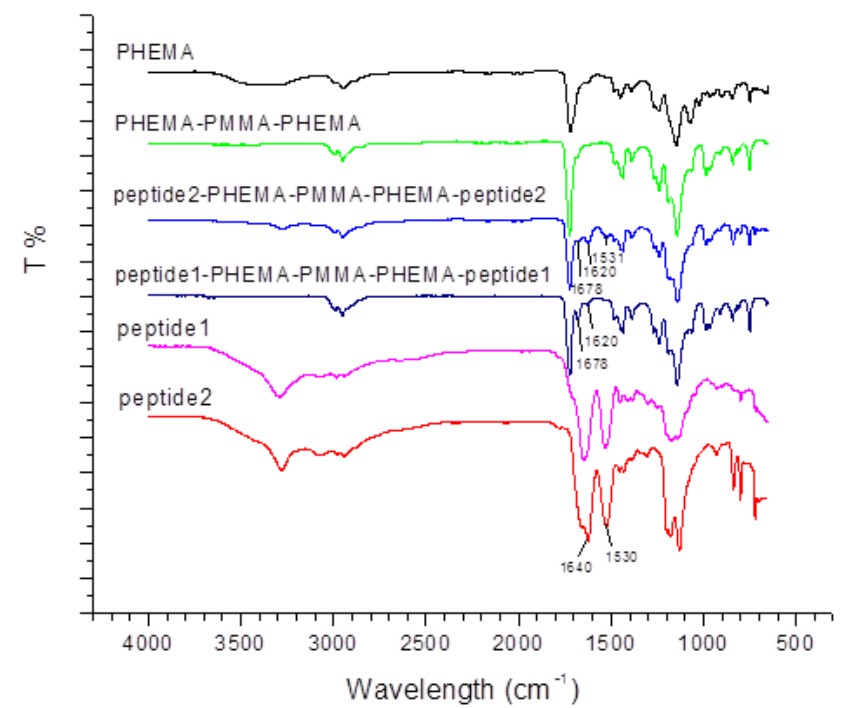

(a)

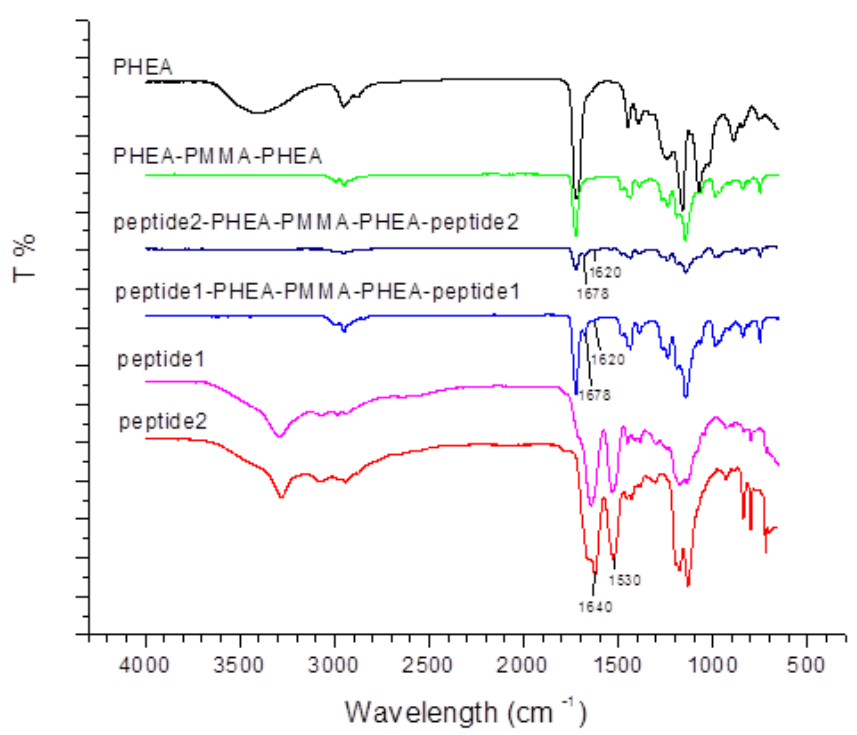

(b)

Figure 2.4S. Comparison of FT-IR spectra of the synthesized copolymer, P1(Peptide1), P2(Peptide2), and Peptide-copolymer conjugates. (a) Comparison of IR spectra of P1(Peptide1), P2(Peptide2) and Peptide- PHEMA-b-12PMMA-b-PHEMA conjugates. (b) Comparison of IR spectra of P1(Peptide1), P2(Peptide2), and PeptidePHEA-b-12PMMA-b-PHEA conjugates. 


\section{Appendix B}

\section{Supplementary Data for Chapter 3}

Smart Self-Assembly of Peptide-Functionalized Amphiphilic Copolymer

Nanoparticles: Novel Fibers and 3D Scaffolds with Ability for Multiple

Drug Loading and Independent Controlled Release

XiaochuDing ${ }^{\mathrm{a}}$, Trevor Moser ${ }^{\mathrm{b}}$, Qi Gao ${ }^{\mathrm{b}}$, TolouShokuhfar ${ }^{\mathrm{b}}$, Patricia A. Heiden ${ }^{\mathrm{a} *}$,

aDepartment of Chemistry, Michigan Technological University, Houghton, MI 49931, USA

${ }^{b}$ Department of Mechanical Engineering and Engineering Mechanics, Michigan Technological University, Houghton, MI 49931, USA

*Corresponding author: +1 906487 3452; fax: +1 9064872061.

Email address:paheiden@mtu.edu (P.A. Heiden).

1. Calibration curve for quantitative analysis of controlled release using different model drugs

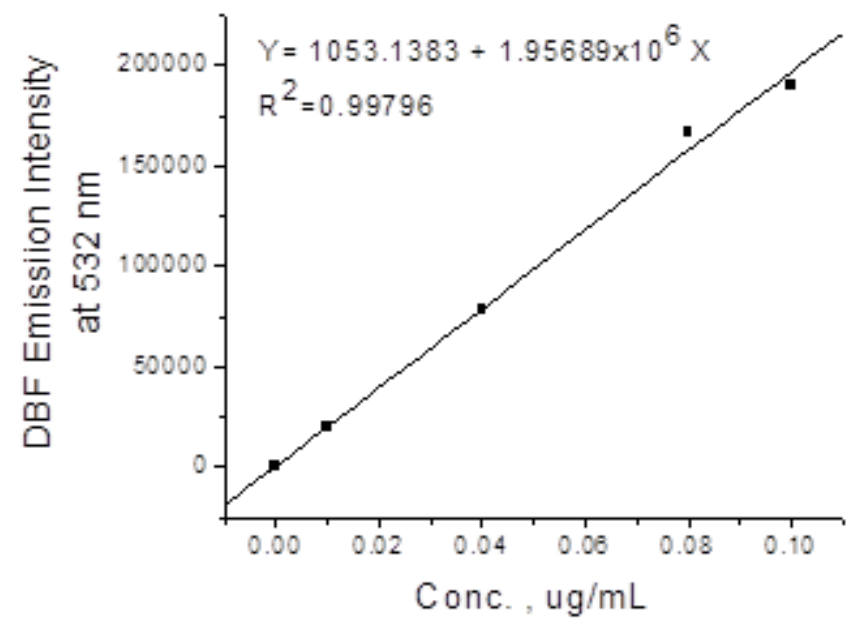

Figure 3.1S. Calibration curve of DBF used for quantitative determination of uncaptured DBF and controlled released DBF sample solutions. 


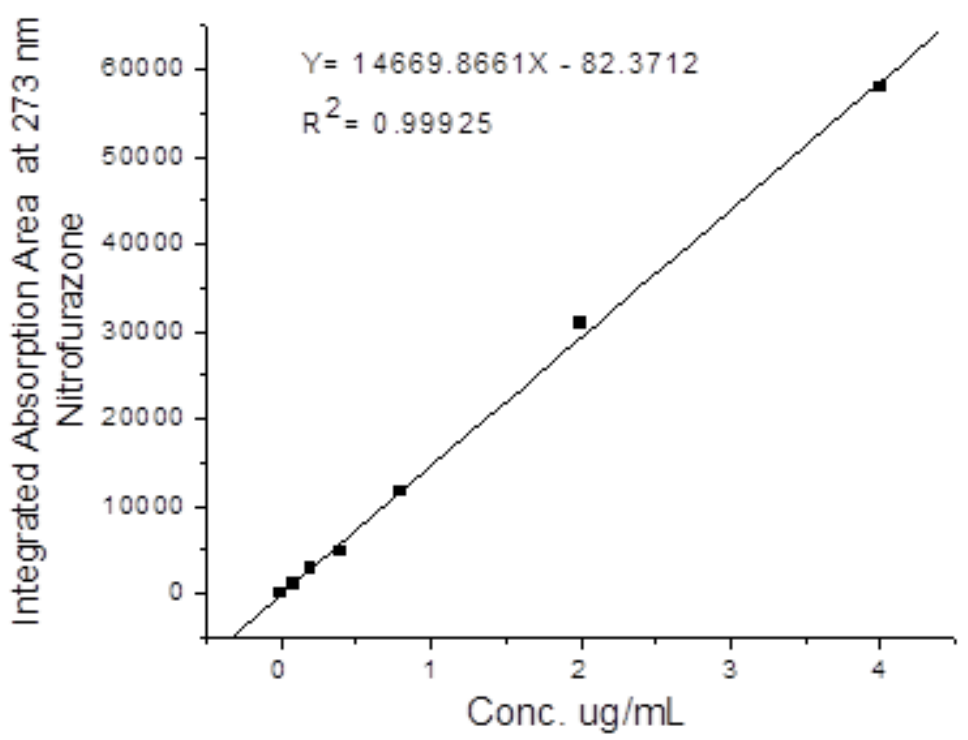

(a)

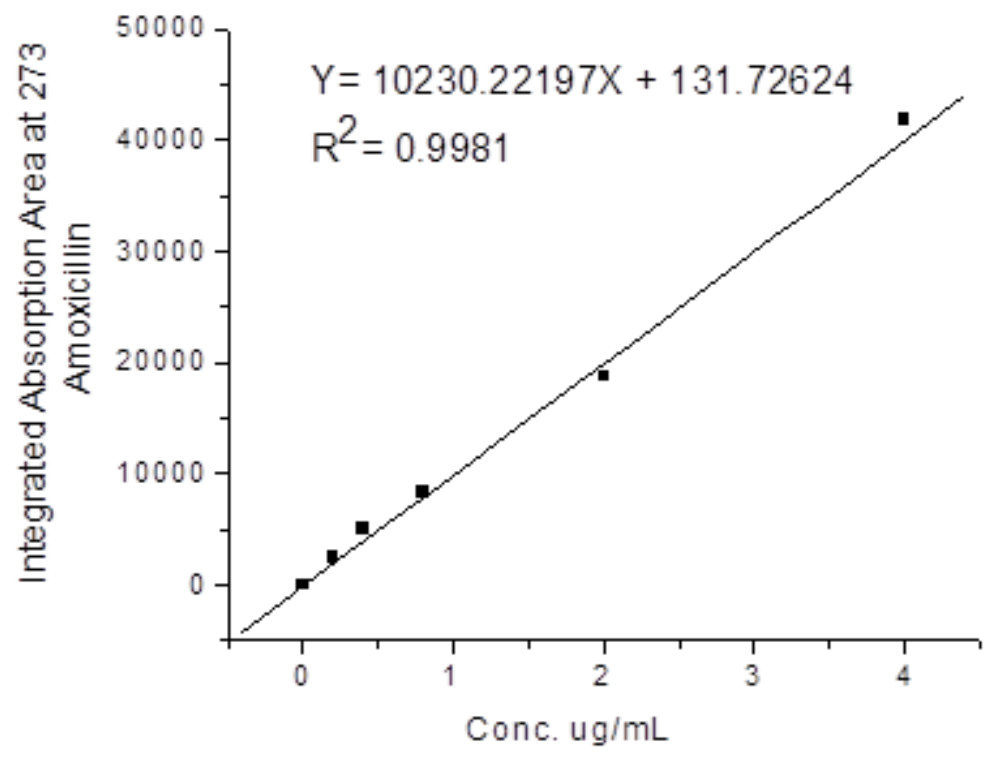

(b)

Figure 3.2S. Calibration curves of (a) nitrofurazone and (b) amoxicillin used for quantitative determination of uncaptured model drugs and simultaneously released model drugs(nitrofurazone and amoxicillin) from same scaffold. 


\section{Appendix C}

\section{Peptide Synthesis Program}

\section{Peptide 1: TTTT-AEAEAEAE}

$\mathrm{T}=$ Threonine, $\mathrm{A}=$ Alanine, $\mathrm{E}=$ Glutamic Acid (negative charge between $\mathrm{pH}$ 4.4-10)

Use Endeavor 90 peptide synthesizer (AAPPTec LLC) to synthesize peptide 1. It is conducted on a $0.65 \mathrm{mmol} / \mathrm{g}$ Rink Fmoc-amide resin $(0.65 \mathrm{mmol}$ amide groups). Aminno acid is made in $4 \times$ excess to Rink Fmoc-amide resin $(4 \times 0.65 \mathrm{mmol}$ amino acid in 5.2 $\mathrm{mL}$ of $0.9 \mathrm{M}$ DIPEA/DMF solution for coupling each amino acid).

Synthesizing Program:

1. ** swelling \& deprotection**

2. Wash RV1 with $10.00 \mathrm{~mL}$ from DMF 2 times.

3. Fill RV1 with $25.00 \mathrm{~mL}$ from DMF.

4. Blank.

5. Mix RV1 via Mechanical Mix for 45 minutes.

6. Empty RV1.

7. Fill RV1 with $15.00 \mathrm{~mL}$ from PIP.

8. Mix RV1 via Mech/N2 Mix for 5.00 minutes.

9. Empty RV1.

10. Blank.

11. Fill RV1 with $15.00 \mathrm{~mL}$ from PIP.

12. Mix RV1 via Mech/N2 Mix for 20 minutes.

13. Empty RV1.

14. Blank.

15. ** clean PIP from the fluid lines**

16. Clean lines with $50.00 \mathrm{~mL}$ from DMF.

17. Blank.

18. ** wash Rink resin**

19. Wash RV1 with $10 \mathrm{~mL}$ from DMF 4 Times.

20. Fill RV1 with $10 \mathrm{~mL}$ from DMF.

21. Delay 5.00 minutes.

22. Empty RV1.

23. Blank.

24. Blank.

25. ** Fmoc-Glutamic Acid $1 * *$

26. Dissolve AA1 with $5.20 \mathrm{~mL}$ from DIPEA for 5.00 minutes.

27. Fill RV1 with $5.20 \mathrm{~mL}$ from AA1. 
28. Mix RV1 via Mech/N2 Mix for 30.00 minutes.

29. Empty RV1.

30. Fill RV1 with $10.00 \mathrm{~mL}$ from DMF.

31. Wash RV1 with $10.00 \mathrm{~mL}$ from DMF 4 Times.

32. Fill RV1 with $10 \mathrm{~mL}$ from DMF.

33. Delay 5.00 minutes.

34. Empty RV1.

35. Fill RV1 with $15.00 \mathrm{~mL}$ from PIP.

36. Mix RV1 via Mech/N2 Mix for 5 minutes.

37. Empty RV1.

38. Fill RV1 with $15.00 \mathrm{~mL}$ from PIP.

39. Mix RV1 via Mech/N2 Mix for 20 minutes.

40. Empty RV1.

41. Blank.

42. Fill RV1 with $10.00 \mathrm{~mL}$ from DMF.

43. Wash RV1 with $10.00 \mathrm{~mL}$ from DMF 4 Times.

44. Fill RV1 with $10 \mathrm{~mL}$ from DMF.

45. Delay 5.00 minutes.

46. Empty RV1.

47. Blank.

48. Blank.

49. ** Fmoc-Alanine 2**

50. Dissolve AA2 with $5.20 \mathrm{~mL}$ from DIPEA for 5.00 minutes.

51. Fill RV1 with $5.20 \mathrm{~mL}$ from AA2.

52. Mix RV1 via Mech/N2 Mix for 30.00 minutes.

53. Empty RV1.

54. Fill RV1 with $10.00 \mathrm{~mL}$ from DMF.

55. Wash RV1 with $10.00 \mathrm{~mL}$ from DMF 4 Times.

56. Fill RV1 with $10 \mathrm{~mL}$ from DMF.

57. Delay 5.00 minutes.

58. Empty RV1.

59. Fill RV1 with $15.00 \mathrm{~mL}$ from PIP.

60. Mix RV1 via Mech/N2 Mix for 5 minutes.

61. Empty RV1.

62. Fill RV1 with $15.00 \mathrm{~mL}$ from PIP.

63. Mix RV1 via Mech/N2 Mix for 20 minutes.

64. Empty RV1.

65. Blank.

66. Fill RV1 with $10.00 \mathrm{~mL}$ from DMF.

67. Wash RV1 with $10.00 \mathrm{~mL}$ from DMF 4 Times. 
68. Fill RV1 with $10 \mathrm{~mL}$ from DMF.

69. Delay 5.00 minutes.

70. Empty RV1.

71. Blank.

72. Blank.

73.** Fmoc-Glutamic acid 3**

74. Dissolve AA3 with $5.20 \mathrm{~mL}$ from DIPEA for 5.00 minutes.

75. Fill RV1 with $5.20 \mathrm{~mL}$ from AA3.

76. Mix RV1 via Mech/N2 Mix for 30.00 minutes.

77. Empty RV1.

78. Fill RV1 with $10.00 \mathrm{~mL}$ from DMF.

79. Wash RV1 with $10.00 \mathrm{~mL}$ from DMF 4 Times.

80. Fill RV1 with $10 \mathrm{~mL}$ from DMF.

81. Delay 5.00 minutes.

82. Empty RV1.

83. Fill RV1 with $15.00 \mathrm{~mL}$ from PIP.

84. Mix RV1 via Mech/N2 Mix for 5 minutes.

85. Empty RV1.

86. Fill RV1 with $15.00 \mathrm{~mL}$ from PIP.

87. Mix RV1 via Mech/N2 Mix for 20 minutes.

88. Empty RV1.

89. Blank.

90. Fill RV1 with $10.00 \mathrm{~mL}$ from DMF.

91. Wash RV1 with $10.00 \mathrm{~mL}$ from DMF 4 Times.

92. Fill RV1 with $10 \mathrm{~mL}$ from DMF.

93. Delay 5.00 minutes.

94. Empty RV1.

95. Blank.

96. Blank.

97.** Fmoc-Alanine 4**

98. Dissolve AA4 with $5.20 \mathrm{~mL}$ from DIPEA for 5.00 minutes.

99. Fill RV1 with $5.20 \mathrm{~mL}$ from AA4.

100. Mix RV1 via Mech/N2 Mix for 30.00 minutes.

101. Empty RV1.

102. Fill RV1 with $10.00 \mathrm{~mL}$ from DMF.

103. Wash RV1 with $10.00 \mathrm{~mL}$ from DMF 4 Times.

104. Fill RV1 with $10 \mathrm{~mL}$ from DMF.

105. Delay 5.00 minutes.

106. Empty RV1.

107. Fill RV1 with $15.00 \mathrm{~mL}$ from PIP. 
108. Mix RV1 via Mech/N2 Mix for 5 minutes.

109. Empty RV1.

110. Fill RV1 with $15.00 \mathrm{~mL}$ from PIP.

111. Mix RV1 via Mech/N2 Mix for 20 minutes.

112. Empty RV1.

113. Blank.

114. Fill RV1 with $10.00 \mathrm{~mL}$ from DMF.

115. Wash RV1 with $10.00 \mathrm{~mL}$ from DMF 4 Times.

116. Fill RV1 with $10 \mathrm{~mL}$ from DMF.

117. Delay 5.00 minutes.

118. Empty RV1.

119. Blank.

120. Pause.

121. ** Fmoc-Glutamic Acid 5**

122. Dissolve AA1 with $5.20 \mathrm{~mL}$ from DIPEA for 5.00 minutes.

123. Fill RV1 with $5.20 \mathrm{~mL}$ from AA1.

124. Mix RV1 via Mech/N2 Mix for 30.00 minutes.

125. Empty RV1.

126. Fill RV1 with $10.00 \mathrm{~mL}$ from DMF.

127. Wash RV1 with $10.00 \mathrm{~mL}$ from DMF 4 Times.

128. Fill RV1 with $10 \mathrm{~mL}$ from DMF.

129. Delay 5.00 minutes.

130. Empty RV1.

131. Fill RV1 with $15.00 \mathrm{~mL}$ from PIP.

132. Mix RV1 via Mech/N2 Mix for 5 minutes.

133. Empty RV1.

134. Fill RV1 with $15.00 \mathrm{~mL}$ from PIP.

135. Mix RV1 via Mech/N2 Mix for 20 minutes.

136. Empty RV1.

137. Blank.

138. Fill RV1 with $10.00 \mathrm{~mL}$ from DMF.

139. Wash RV1 with $10.00 \mathrm{~mL}$ from DMF 4 Times.

140. Fill RV1 with $10 \mathrm{~mL}$ from DMF.

141. Delay 5.00 minutes.

142. Empty RV1.

143. Blank.

144. Blank.

145. ** Fmoc-Alanine $6 * *$

146. Dissolve AA2 with $5.20 \mathrm{~mL}$ from DIPEA for 5.00 minutes. 
147. Fill RV1 with $5.20 \mathrm{~mL}$ from AA2.

148. Mix RV1 via Mech/N2 Mix for 30.00 minutes.

149. Empty RV1.

150. Fill RV1 with $10.00 \mathrm{~mL}$ from DMF.

151. Wash RV1 with $10.00 \mathrm{~mL}$ from DMF 4 Times.

152. Fill RV1 with $10 \mathrm{~mL}$ from DMF.

153. Delay 5.00 minutes.

154. Empty RV1.

155. Fill RV1 with $15.00 \mathrm{~mL}$ from PIP.

156. Mix RV1 via Mech/N2 Mix for 5 minutes.

157. Empty RV1.

158. Fill RV1 with $15.00 \mathrm{~mL}$ from PIP.

159. Mix RV1 via Mech/N2 Mix for 20 minutes.

160. Empty RV1.

161. Blank.

162. Fill RV1 with $10.00 \mathrm{~mL}$ from DMF.

163. Wash RV1 with $10.00 \mathrm{~mL}$ from DMF 4 Times.

164. Fill RV1 with $10 \mathrm{~mL}$ from DMF.

165. Delay 5.00 minutes.

166. Empty RV1.

167. Blank.

168. Blank.

169.** Fmoc-Glutamic acid $7 * *$

170. Dissolve AA3 with $5.20 \mathrm{~mL}$ from DIPEA for 5.00 minutes.

171. Fill RV1 with $5.20 \mathrm{~mL}$ from AA3.

172. Mix RV1 via Mech/N2 Mix for 30.00 minutes.

173. Empty RV1.

174. Fill RV1 with $10.00 \mathrm{~mL}$ from DMF.

175. Wash RV1 with $10.00 \mathrm{~mL}$ from DMF 4 Times.

176. Fill RV1 with $10 \mathrm{~mL}$ from DMF.

177. Delay 5.00 minutes.

178. Empty RV1.

179. Fill RV1 with $15.00 \mathrm{~mL}$ from PIP.

180. Mix RV1 via Mech/N2 Mix for 5 minutes.

181. Empty RV1.

182. Fill RV1 with $15.00 \mathrm{~mL}$ from PIP.

183. Mix RV1 via Mech/N2 Mix for 20 minutes.

184. Empty RV1.

185. Blank.

186. Fill RV1 with $10.00 \mathrm{~mL}$ from DMF. 
187. Wash RV1 with $10.00 \mathrm{~mL}$ from DMF 4 Times.

188. Fill RV1 with $10 \mathrm{~mL}$ from DMF.

189. Delay 5.00 minutes.

190. Empty RV1.

191. Blank.

192. Blank.

193.** Fmoc-Alanine 8**

194. Dissolve AA4 with $5.20 \mathrm{~mL}$ from DIPEA for 5.00 minutes.

195. Fill RV1 with $5.20 \mathrm{~mL}$ from AA4.

196. Mix RV1 via Mech/N2 Mix for 30.00 minutes.

197. Empty RV1.

198. Fill RV1 with $10.00 \mathrm{~mL}$ from DMF.

199. Wash RV1 with $10.00 \mathrm{~mL}$ from DMF 4 Times.

200. Fill RV1 with $10 \mathrm{~mL}$ from DMF.

201. Delay 5.00 minutes.

202. Empty RV1.

203. Fill RV1 with $15.00 \mathrm{~mL}$ from PIP.

204. Mix RV1 via Mech/N2 Mix for 5 minutes.

205. Empty RV1.

206. Fill RV1 with $15.00 \mathrm{~mL}$ from PIP.

207. Mix RV1 via Mech/N2 Mix for 20 minutes.

208. Empty RV1.

209. Blank.

210. Fill RV1 with $10.00 \mathrm{~mL}$ from DMF.

211. Wash RV1 with $10.00 \mathrm{~mL}$ from DMF 4 Times.

212. Fill RV1 with $10 \mathrm{~mL}$ from DMF.

213. Delay 5.00 minutes.

214. Empty RV1.

215. Blank.

216. Pause.

217** Fmoc-Threonine 9**

218. Dissolve AA1 with $5.20 \mathrm{~mL}$ from DIPEA for 5.00 minutes.

219. Fill RV1 with $5.20 \mathrm{~mL}$ from AA1.

220. Mix RV1 via Mech/N2 Mix for 30.00 minutes.

221. Empty RV1.

222. Fill RV1 with $10.00 \mathrm{~mL}$ from DMF.

223. Wash RV1 with $10.00 \mathrm{~mL}$ from DMF 4 Times.

224. Fill RV1 with $10 \mathrm{~mL}$ from DMF.

225. Delay 5.00 minutes.

226. Empty RV1. 
227. Fill RV1 with $15.00 \mathrm{~mL}$ from PIP.

228. Mix RV1 via Mech/N2 Mix for 5 minutes.

229. Empty RV1.

230. Fill RV1 with $15.00 \mathrm{~mL}$ from PIP.

231. Mix RV1 via Mech/N2 Mix for 20 minutes.

232. Empty RV1.

233. Blank.

234. Fill RV1 with $10.00 \mathrm{~mL}$ from DMF.

235. Wash RV1 with $10.00 \mathrm{~mL}$ from DMF 4 Times.

236. Fill RV1 with $10 \mathrm{~mL}$ from DMF.

237. Delay 5.00 minutes.

238. Empty RV1.

239. Blank.

240. Blank.

241. ** Fmoc-Threonine $10 * *$

242. Dissolve AA2 with $5.20 \mathrm{~mL}$ from DIPEA for 5.00 minutes.

243. Fill RV1 with $5.20 \mathrm{~mL}$ from AA2.

244. Mix RV1 via Mech/N2 Mix for 30.00 minutes.

245. Empty RV1.

246. Fill RV1 with $10.00 \mathrm{~mL}$ from DMF.

247. Wash RV1 with $10.00 \mathrm{~mL}$ from DMF 4 Times.

248. Fill RV1 with $10 \mathrm{~mL}$ from DMF.

249. Delay 5.00 minutes.

250. Empty RV1.

251. Fill RV1 with $15.00 \mathrm{~mL}$ from PIP.

252. Mix RV1 via Mech/N2 Mix for 5 minutes.

253. Empty RV1.

254. Fill RV1 with $15.00 \mathrm{~mL}$ from PIP.

255. Mix RV1 via Mech/N2 Mix for 20 minutes.

256. Empty RV1.

257. Blank.

258. Fill RV1 with $10.00 \mathrm{~mL}$ from DMF.

259. Wash RV1 with $10.00 \mathrm{~mL}$ from DMF 4 Times.

260. Fill RV1 with $10 \mathrm{~mL}$ from DMF.

261. Delay 5.00 minutes.

262. Empty RV1.

263. Blank.

264. Blank.

265.** Fmoc-Threonine 11**

266. Dissolve AA3 with $5.20 \mathrm{~mL}$ from DIPEA for 5.00 minutes. 
267. Fill RV1 with $5.20 \mathrm{~mL}$ from AA3.

268. Mix RV1 via Mech/N2 Mix for 30.00 minutes.

269. Empty RV1.

270. Fill RV1 with $10.00 \mathrm{~mL}$ from DMF.

270. Wash RV1 with $10.00 \mathrm{~mL}$ from DMF 4 Times.

272. Fill RV1 with $10 \mathrm{~mL}$ from DMF.

273. Delay 5.00 minutes.

274. Empty RV1.

275. Fill RV1 with $15.00 \mathrm{~mL}$ from PIP.

276. Mix RV1 via Mech/N2 Mix for 5 minutes.

277. Empty RV1.

278. Fill RV1 with $15.00 \mathrm{~mL}$ from PIP.

279. Mix RV1 via Mech/N2 Mix for 20 minutes.

280. Empty RV1.

281. Blank.

282. Fill RV1 with $10.00 \mathrm{~mL}$ from DMF.

283. Wash RV1 with $10.00 \mathrm{~mL}$ from DMF 4 Times.

284. Fill RV1 with $10 \mathrm{~mL}$ from DMF.

285. Delay 5.00 minutes.

286. Empty RV1.

287. Blank.

288. Blank.

289.** Fmoc-Threonine 12**

290. Dissolve AA4 with $5.20 \mathrm{~mL}$ from DIPEA for 5.00 minutes.

291. Fill RV1 with $5.20 \mathrm{~mL}$ from AA4.

292. Mix RV1 via Mech/N2 Mix for 30.00 minutes.

293. Empty RV1.

294. Fill RV1 with $10.00 \mathrm{~mL}$ from DMF.

295. Wash RV1 with $10.00 \mathrm{~mL}$ from DMF 4 Times.

296. Fill RV1 with $10 \mathrm{~mL}$ from DMF.

297. Delay 5.00 minutes.

298. Empty RV1.

299. Fill RV1 with $15.00 \mathrm{~mL}$ from PIP.

300. Mix RV1 via Mech/N2 Mix for 5 minutes.

301. Empty RV1.

302. Fill RV1 with $15.00 \mathrm{~mL}$ from PIP.

303. Mix RV1 via Mech/N2 Mix for 20 minutes.

304. Empty RV1.

305. Blank.

306. Fill RV1 with $10.00 \mathrm{~mL}$ from DMF. 
307. Wash RV1 with $10.00 \mathrm{~mL}$ from DMF 4 Times.

308. Fill RV1 with $10 \mathrm{~mL}$ from DMF.

309. Delay 5.00 minutes.

310. Empty RV1.

311. Blank.

312. Blank.

313.** Solvent switch \& Storage**

314. Fill RV1 with $10.00 \mathrm{~mL}$ from DCM.

315. Wash RV1 with $10.00 \mathrm{~mL}$ from DCM 4 Times.

316. Fill RV1 with $10.00 \mathrm{~mL}$ from DCM.

317. Delay for 5.00 minutes.

318. Empty RV1.

319. Blank. 


\section{Peptide 2: TTTT-AKAKAKAK}

$\mathrm{T}=$ Threonine, $\mathrm{A}=$ Alanine, $\mathrm{K}=$ Lysine (Positive charge between $\mathrm{pH}$ 4.4 10)

Use Endeavor 90 peptide synthesizer (AAPPTec LLC) to synthesize peptide 2. It is conducted on a $0.65 \mathrm{mmol} / \mathrm{g}$ Rink Fmoc-amide resin $(0.65 \mathrm{mmol}$ amide groups). Aminno acid is made in $4 \times$ excess to Rink Fmoc-amide resin $(4 \times 0.65 \mathrm{mmol}$ amino acid in $5.2 \mathrm{~mL}$ of $0.9 \mathrm{M}$ DIPEA/DMF solution for coupling each amino acid).

Synthesizing Program:

1. ** swelling \& deprotection**

2. Wash RV1 with $10.00 \mathrm{~mL}$ from DMF 2 times.

3. Fill RV1 with $25.00 \mathrm{~mL}$ from DMF.

4. Blank.

5. Mix RV1 via Mechanical Mix for 45 minutes.

6. Empty RV1.

7. Fill RV1 with $15.00 \mathrm{~mL}$ from PIP.

8. Mix RV1 via Mech/N2 Mix for 5.00 minutes.

9. Empty RV1.

10. Blank.

11. Fill RV1 with $15.00 \mathrm{~mL}$ from PIP.

12. Mix RV1 via Mech/N2 Mix for 20 minutes.

13. Empty RV1.

14. Blank.

15. ** clean PIP from the fluid lines**

16. Clean lines with $50.00 \mathrm{~mL}$ from DMF.

17. Blank.

18. ** wash Rink resin**

19. Wash RV1 with $10 \mathrm{~mL}$ from DMF 4 Times.

20. Fill RV1 with $10 \mathrm{~mL}$ from DMF.

21. Delay 5.00 minutes.

22. Empty RV1.

23. Blank.

24. Blank.

25. ** Fmoc-Lysine 1**

26. Dissolve AA1 with $5.20 \mathrm{~mL}$ from DIPEA for 5.00 minutes.

27. Fill RV1 with $5.20 \mathrm{~mL}$ from AA1.

28. Mix RV1 via Mech/N2 Mix for 30.00 minutes.

29. Empty RV1. 
30. Fill RV1 with $10.00 \mathrm{~mL}$ from DMF.

31. Wash RV1 with $10.00 \mathrm{~mL}$ from DMF 4 Times.

32. Fill RV1 with $10 \mathrm{~mL}$ from DMF.

33. Delay 5.00 minutes.

34. Empty RV1.

35. Fill RV1 with $15.00 \mathrm{~mL}$ from PIP.

36. Mix RV1 via Mech/N2 Mix for 5 minutes.

37. Empty RV1.

38. Fill RV1 with $15.00 \mathrm{~mL}$ from PIP.

39. Mix RV1 via Mech/N2 Mix for 20 minutes.

40. Empty RV1.

41. Blank.

42. Fill RV1 with $10.00 \mathrm{~mL}$ from DMF.

43. Wash RV1 with $10.00 \mathrm{~mL}$ from DMF 4 Times.

44. Fill RV1 with $10 \mathrm{~mL}$ from DMF.

45. Delay 5.00 minutes.

46. Empty RV1.

47. Blank.

48. Blank.

49. ** Fmoc-Alanine $2 * *$

50. Dissolve AA2 with $5.20 \mathrm{~mL}$ from DIPEA for 5.00 minutes.

51. Fill RV1 with $5.20 \mathrm{~mL}$ from AA2.

52. Mix RV1 via Mech/N2 Mix for 30.00 minutes.

53. Empty RV1.

54. Fill RV1 with $10.00 \mathrm{~mL}$ from DMF.

55. Wash RV1 with $10.00 \mathrm{~mL}$ from DMF 4 Times.

56. Fill RV1 with $10 \mathrm{~mL}$ from DMF.

57. Delay 5.00 minutes.

58. Empty RV1.

59. Fill RV1 with $15.00 \mathrm{~mL}$ from PIP.

60. Mix RV1 via Mech/N2 Mix for 5 minutes.

61. Empty RV1.

62. Fill RV1 with $15.00 \mathrm{~mL}$ from PIP.

63. Mix RV1 via Mech/N2 Mix for 20 minutes.

64. Empty RV1.

65. Blank.

66. Fill RV1 with $10.00 \mathrm{~mL}$ from DMF.

67. Wash RV1 with $10.00 \mathrm{~mL}$ from DMF 4 Times.

68. Fill RV1 with $10 \mathrm{~mL}$ from DMF.

69. Delay 5.00 minutes. 
70. Empty RV1.

71. Blank.

72. Blank.

73. ** Fmoc-Lysine 3**

74. Dissolve AA3 with $5.20 \mathrm{~mL}$ from DIPEA for 5.00 minutes.

75. Fill RV1 with $5.20 \mathrm{~mL}$ from AA3.

76. Mix RV1 via Mech/N2 Mix for 30.00 minutes.

77. Empty RV1.

78. Fill RV1 with $10.00 \mathrm{~mL}$ from DMF.

79. Wash RV1 with $10.00 \mathrm{~mL}$ from DMF 4 Times.

80. Fill RV1 with $10 \mathrm{~mL}$ from DMF.

81. Delay 5.00 minutes.

82. Empty RV1.

83. Fill RV1 with $15.00 \mathrm{~mL}$ from PIP.

84. Mix RV1 via Mech/N2 Mix for 5 minutes.

85. Empty RV1.

86. Fill RV1 with $15.00 \mathrm{~mL}$ from PIP.

87. Mix RV1 via Mech/N2 Mix for 20 minutes.

88. Empty RV1.

89. Blank.

90. Fill RV1 with $10.00 \mathrm{~mL}$ from DMF.

91. Wash RV1 with $10.00 \mathrm{~mL}$ from DMF 4 Times.

92. Fill RV1 with $10 \mathrm{~mL}$ from DMF.

93. Delay 5.00 minutes.

94. Empty RV1.

95. Blank.

96. Blank.

97.** Fmoc-Alanine $4 * *$

98. Dissolve AA4 with $5.20 \mathrm{~mL}$ from DIPEA for 5.00 minutes.

99. Fill RV1 with $5.20 \mathrm{~mL}$ from AA4.

100. Mix RV1 via Mech/N2 Mix for 30.00 minutes.

101. Empty RV1.

102. Fill RV1 with $10.00 \mathrm{~mL}$ from DMF.

103. Wash RV1 with $10.00 \mathrm{~mL}$ from DMF 4 Times.

104. Fill RV1 with $10 \mathrm{~mL}$ from DMF.

105. Delay 5.00 minutes.

106. Empty RV1.

107. Fill RV1 with $15.00 \mathrm{~mL}$ from PIP.

108. Mix RV1 via Mech/N2 Mix for 5 minutes.

109. Empty RV1. 
110. Fill RV1 with $15.00 \mathrm{~mL}$ from PIP.

111. Mix RV1 via Mech/N2 Mix for 20 minutes.

112. Empty RV1.

113. Blank.

114. Fill RV1 with $10.00 \mathrm{~mL}$ from DMF.

115. Wash RV1 with $10.00 \mathrm{~mL}$ from DMF 4 Times.

116. Fill RV1 with $10 \mathrm{~mL}$ from DMF.

117. Delay 5.00 minutes.

118. Empty RV1.

119. Blank.

120. Pause.

121. ** Fmoc-Lysine $5 * *$

122. Dissolve AA1 with $5.20 \mathrm{~mL}$ from DIPEA for 5.00 minutes.

123. Fill RV1 with $5.20 \mathrm{~mL}$ from AA1.

124. Mix RV1 via Mech/N2 Mix for 30.00 minutes.

125. Empty RV1.

126. Fill RV1 with $10.00 \mathrm{~mL}$ from DMF.

127. Wash RV1 with $10.00 \mathrm{~mL}$ from DMF 4 Times.

128. Fill RV1 with $10 \mathrm{~mL}$ from DMF.

129. Delay 5.00 minutes.

130. Empty RV1.

131. Fill RV1 with $15.00 \mathrm{~mL}$ from PIP.

132. Mix RV1 via Mech/N2 Mix for 5 minutes.

133. Empty RV1.

134. Fill RV1 with $15.00 \mathrm{~mL}$ from PIP.

135. Mix RV1 via Mech/N2 Mix for 20 minutes.

136. Empty RV1.

137. Blank.

138. Fill RV1 with $10.00 \mathrm{~mL}$ from DMF.

139. Wash RV1 with $10.00 \mathrm{~mL}$ from DMF 4 Times.

140. Fill RV1 with $10 \mathrm{~mL}$ from DMF.

141. Delay 5.00 minutes.

142. Empty RV1.

143. Blank.

144. Blank.

145. ** Fmoc-Alanine $6 * *$

146. Dissolve AA2 with $5.20 \mathrm{~mL}$ from DIPEA for 5.00 minutes.

147. Fill RV1 with $5.20 \mathrm{~mL}$ from AA2.

148. Mix RV1 via Mech/N2 Mix for 30.00 minutes. 
149. Empty RV1.

150. Fill RV1 with $10.00 \mathrm{~mL}$ from DMF.

151. Wash RV1 with $10.00 \mathrm{~mL}$ from DMF 4 Times.

152. Fill RV1 with $10 \mathrm{~mL}$ from DMF.

153. Delay 5.00 minutes.

154. Empty RV1.

155. Fill RV1 with $15.00 \mathrm{~mL}$ from PIP.

156. Mix RV1 via Mech/N2 Mix for 5 minutes.

157. Empty RV1.

158. Fill RV1 with $15.00 \mathrm{~mL}$ from PIP.

159. Mix RV1 via Mech/N2 Mix for 20 minutes.

160. Empty RV1.

161. Blank.

162. Fill RV1 with $10.00 \mathrm{~mL}$ from DMF.

163. Wash RV1 with $10.00 \mathrm{~mL}$ from DMF 4 Times.

164. Fill RV1 with $10 \mathrm{~mL}$ from DMF.

165. Delay 5.00 minutes.

166. Empty RV1.

167. Blank.

168. Blank.

169.** Fmoc-Lysine 7**

170. Dissolve AA3 with $5.20 \mathrm{~mL}$ from DIPEA for 5.00 minutes.

171. Fill RV1 with $5.20 \mathrm{~mL}$ from AA3.

172. Mix RV1 via Mech/N2 Mix for 30.00 minutes.

173. Empty RV1.

174. Fill RV1 with $10.00 \mathrm{~mL}$ from DMF.

175. Wash RV1 with $10.00 \mathrm{~mL}$ from DMF 4 Times.

176. Fill RV1 with $10 \mathrm{~mL}$ from DMF.

177. Delay 5.00 minutes.

178. Empty RV1.

179. Fill RV1 with $15.00 \mathrm{~mL}$ from PIP.

180. Mix RV1 via Mech/N2 Mix for 5 minutes.

181. Empty RV1.

182. Fill RV1 with $15.00 \mathrm{~mL}$ from PIP.

183. Mix RV1 via Mech/N2 Mix for 20 minutes.

184. Empty RV1.

185. Blank.

186. Fill RV1 with $10.00 \mathrm{~mL}$ from DMF.

187. Wash RV1 with $10.00 \mathrm{~mL}$ from DMF 4 Times.

188. Fill RV1 with $10 \mathrm{~mL}$ from DMF. 
189. Delay 5.00 minutes.

190. Empty RV1.

191. Blank.

192. Blank.

193.** Fmoc-Alanine $8 * *$

194. Dissolve AA4 with $5.20 \mathrm{~mL}$ from DIPEA for 5.00 minutes.

195. Fill RV1 with $5.20 \mathrm{~mL}$ from AA4.

196. Mix RV1 via Mech/N2 Mix for 30.00 minutes.

197. Empty RV1.

198. Fill RV1 with $10.00 \mathrm{~mL}$ from DMF.

199. Wash RV1 with $10.00 \mathrm{~mL}$ from DMF 4 Times.

200. Fill RV1 with $10 \mathrm{~mL}$ from DMF.

201. Delay 5.00 minutes.

202. Empty RV1.

203. Fill RV1 with $15.00 \mathrm{~mL}$ from PIP.

204. Mix RV1 via Mech/N2 Mix for 5 minutes.

205. Empty RV1.

206. Fill RV1 with $15.00 \mathrm{~mL}$ from PIP.

207. Mix RV1 via Mech/N2 Mix for 20 minutes.

208. Empty RV1.

209. Blank.

210. Fill RV1 with $10.00 \mathrm{~mL}$ from DMF.

211. Wash RV1 with $10.00 \mathrm{~mL}$ from DMF 4 Times.

212. Fill RV1 with $10 \mathrm{~mL}$ from DMF.

213. Delay 5.00 minutes.

214. Empty RV1.

215. Blank.

216. Pause.

217** Fmoc-Threonine 9**

218. Dissolve AA1 with $5.20 \mathrm{~mL}$ from DIPEA for 5.00 minutes.

219. Fill RV1 with $5.20 \mathrm{~mL}$ from AA1.

220. Mix RV1 via Mech/N2 Mix for 30.00 minutes.

221. Empty RV1.

222. Fill RV1 with $10.00 \mathrm{~mL}$ from DMF.

223. Wash RV1 with $10.00 \mathrm{~mL}$ from DMF 4 Times.

224. Fill RV1 with $10 \mathrm{~mL}$ from DMF.

225. Delay 5.00 minutes.

226. Empty RV1.

227. Fill RV1 with $15.00 \mathrm{~mL}$ from PIP.

228. Mix RV1 via Mech/N2 Mix for 5 minutes. 
229. Empty RV1.

230. Fill RV1 with $15.00 \mathrm{~mL}$ from PIP.

231. Mix RV1 via Mech/N2 Mix for 20 minutes.

232. Empty RV1.

233. Blank.

234. Fill RV1 with $10.00 \mathrm{~mL}$ from DMF.

235. Wash RV1 with $10.00 \mathrm{~mL}$ from DMF 4 Times.

236. Fill RV1 with $10 \mathrm{~mL}$ from DMF.

237. Delay 5.00 minutes.

238. Empty RV1.

239. Blank.

240. Blank.

241. ** Fmoc-Threonine 10**

242. Dissolve AA2 with $5.20 \mathrm{~mL}$ from DIPEA for 5.00 minutes.

243. Fill RV1 with $5.20 \mathrm{~mL}$ from AA2.

244. Mix RV1 via Mech/N2 Mix for 30.00 minutes.

245. Empty RV1.

246. Fill RV1 with $10.00 \mathrm{~mL}$ from DMF.

247. Wash RV1 with $10.00 \mathrm{~mL}$ from DMF 4 Times.

248. Fill RV1 with $10 \mathrm{~mL}$ from DMF.

249. Delay 5.00 minutes.

250. Empty RV1.

251. Fill RV1 with $15.00 \mathrm{~mL}$ from PIP.

252. Mix RV1 via Mech/N2 Mix for 5 minutes.

253. Empty RV1.

254. Fill RV1 with $15.00 \mathrm{~mL}$ from PIP.

255. Mix RV1 via Mech/N2 Mix for 20 minutes.

256. Empty RV1.

257. Blank.

258. Fill RV1 with $10.00 \mathrm{~mL}$ from DMF.

259. Wash RV1 with $10.00 \mathrm{~mL}$ from DMF 4 Times.

260. Fill RV1 with $10 \mathrm{~mL}$ from DMF.

261. Delay 5.00 minutes.

262. Empty RV1.

263. Blank.

264. Blank.

265.** Fmoc-Threonine 11**

266. Dissolve AA3 with $5.20 \mathrm{~mL}$ from DIPEA for 5.00 minutes.

267. Fill RV1 with $5.20 \mathrm{~mL}$ from AA3.

268. Mix RV1 via Mech/N2 Mix for 30.00 minutes. 
269. Empty RV1.

270. Fill RV1 with $10.00 \mathrm{~mL}$ from DMF.

270. Wash RV1 with $10.00 \mathrm{~mL}$ from DMF 4 Times.

272. Fill RV1 with $10 \mathrm{~mL}$ from DMF.

273. Delay 5.00 minutes.

274. Empty RV1.

275. Fill RV1 with $15.00 \mathrm{~mL}$ from PIP.

276. Mix RV1 via Mech/N2 Mix for 5 minutes.

277. Empty RV1.

278. Fill RV1 with $15.00 \mathrm{~mL}$ from PIP.

279. Mix RV1 via Mech/N2 Mix for 20 minutes.

280. Empty RV1.

281. Blank.

282. Fill RV1 with $10.00 \mathrm{~mL}$ from DMF.

283. Wash RV1 with $10.00 \mathrm{~mL}$ from DMF 4 Times.

284. Fill RV1 with $10 \mathrm{~mL}$ from DMF.

285. Delay 5.00 minutes.

286. Empty RV1.

287. Blank.

288. Blank.

289.** Fmoc-Threonine 12**

290. Dissolve AA4 with $5.20 \mathrm{~mL}$ from DIPEA for 5.00 minutes.

291. Fill RV1 with $5.20 \mathrm{~mL}$ from AA4.

292. Mix RV1 via Mech/N2 Mix for 30.00 minutes.

293. Empty RV1.

294. Fill RV1 with $10.00 \mathrm{~mL}$ from DMF.

295. Wash RV1 with $10.00 \mathrm{~mL}$ from DMF 4 Times.

296. Fill RV1 with $10 \mathrm{~mL}$ from DMF.

297. Delay 5.00 minutes.

298. Empty RV1.

299. Fill RV1 with $15.00 \mathrm{~mL}$ from PIP.

300. Mix RV1 via Mech/N2 Mix for 5 minutes.

301. Empty RV1.

302. Fill RV1 with $15.00 \mathrm{~mL}$ from PIP.

303. Mix RV1 via Mech/N2 Mix for 20 minutes.

304. Empty RV1.

305. Blank.

306. Fill RV1 with $10.00 \mathrm{~mL}$ from DMF.

307. Wash RV1 with $10.00 \mathrm{~mL}$ from DMF 4 Times.

308. Fill RV1 with $10 \mathrm{~mL}$ from DMF. 
309. Delay 5.00 minutes.

310. Empty RV1.

311. Blank.

312. Blank.

313.** Solvent switch \& Storage**

314. Fill RV1 with $10.00 \mathrm{~mL}$ from DCM.

315. Wash RV1 with $10.00 \mathrm{~mL}$ from DCM 4 Times.

316. Fill RV1 with $10.00 \mathrm{~mL}$ from DCM.

317. Delay for 5.00 minutes.

318. Empty RV1.

319. Blank. 


\section{Appendix D}

\section{Permission License Number for Chapter 4}

\begin{tabular}{|c|c|}
\hline License Number & 3182031141435 \\
\hline Licensed content publisher & Elsevier \\
\hline $\begin{array}{l}\text { Licensed content } \\
\text { publication }\end{array}$ & Carbohydrate Polymers \\
\hline Licensed content title & $\begin{array}{l}\text { Efficient one-pot synthesis and loading of self-assembled amphiphilic chitosan } \\
\text { nanoparticles for low-leaching wood preservation }\end{array}$ \\
\hline Licensed content author & Xiaochu Ding,Dana L. Richter,Laurent M. Matuana,P.A. Heiden \\
\hline Licensed content date & 1 August 2011 \\
\hline $\begin{array}{l}\text { Licensed content volume } \\
\text { number }\end{array}$ & 86 \\
\hline Type of Use & reuse in a thesis/dissertation \\
\hline Portion & full article \\
\hline Format & electronic \\
\hline $\begin{array}{l}\text { Are you the author of this } \\
\text { Elsevier article? }\end{array}$ & Yes \\
\hline Will you be translating? & No \\
\hline \multicolumn{2}{|l|}{ Order reference number } \\
\hline $\begin{array}{l}\text { Title of your } \\
\text { thesis/dissertation }\end{array}$ & $\begin{array}{l}\text { STUDIES OF FUNCTIONALIZED NANOPARTICLES FOR SMART SELF-ASSEMBLY AND } \\
\text { AS CONTROLLED DRUG DELIVERY }\end{array}$ \\
\hline Total & 0.00 USD \\
\hline
\end{tabular}




\title{
Appendix E
}

\section{Permission License Number for Chapter 5}

\author{
JOHN WILEY AND SONS LICENSE \\ TERMS AND CONDITIONS
}

May 19, 2013

This is a License Agreement between Xiaochu Ding ("You") and John Wiley and Sons ("John Wiley and Sons") provided by Copyright Clearance Center ("CCC"). The license consists of your order details, the terms and conditions provided by John Wiley and Sons, and the payment terms and conditions.

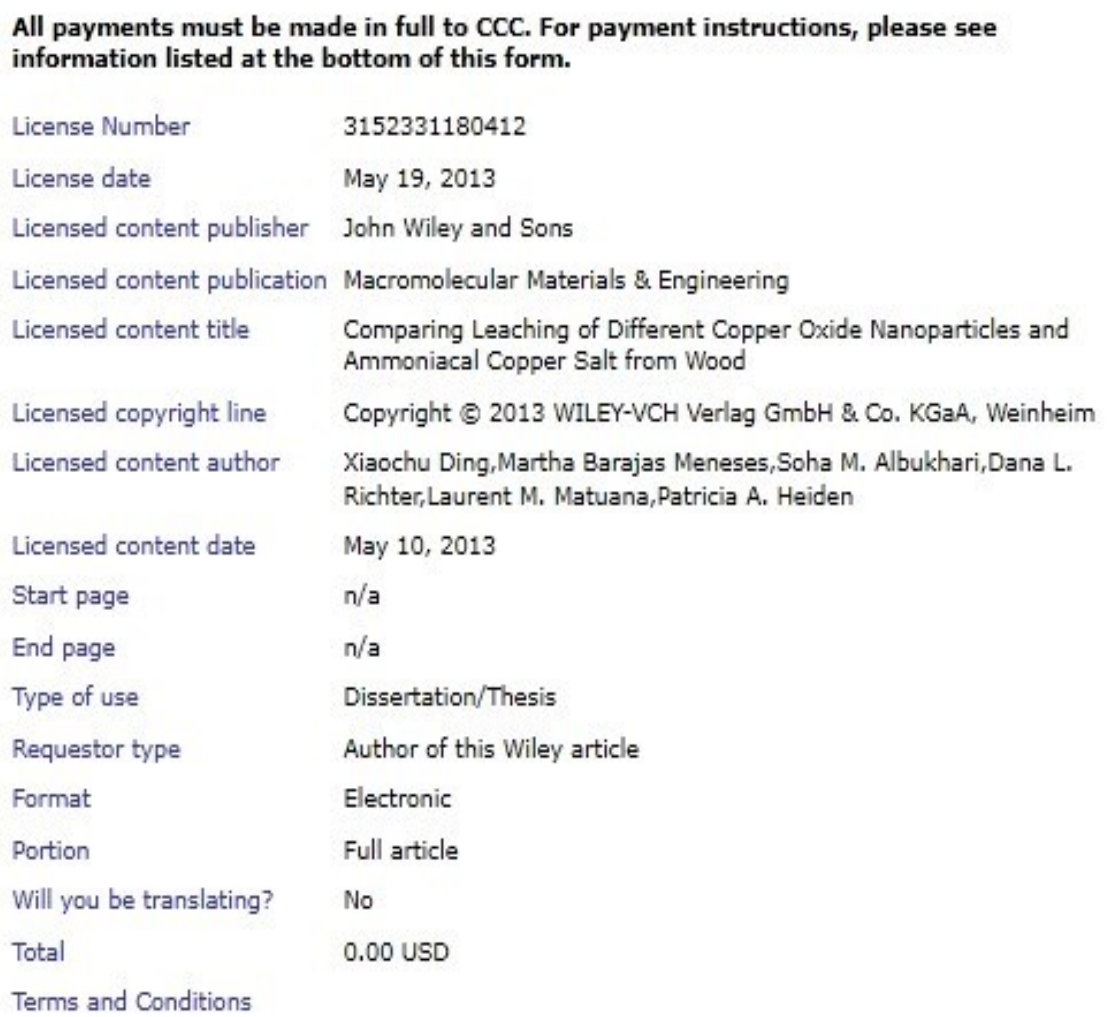

